\title{
IMAGINARY CONE AND REFLECTION SUBGROUPS OF COXETER GROUPS
}

\author{
MATTHEW J. DYER
}

\begin{abstract}
The imaginary cone of a Kac-Moody Lie algebra is the convex hull of zero and the positive imaginary roots. This paper studies the imaginary cone for a class of root systems of general Coxeter groups $W$. It is shown that the imaginary cone of a reflection subgroup of $W$ is contained in that of $W$, and that for irreducible infinite $W$ of finite rank, the closed imaginary cone is the only non-zero, closed, pointed $W$-stable cone contained in the pointed cone spanned by the simple roots. For $W$ of finite rank, various natural notions of faces of the imaginary cone are shown to coincide, the face lattice is explicitly described in terms of the lattice of facial reflection subgroups and it is shown that the Tits cone and imaginary cone are related by a duality closely analogous to the standard duality for polyhedral cones, even though neither of them is a closed cone in general. Some of these results have application, to be given in sequels to this paper, to dominance order of Coxeter groups, associated automata, and construction of modules for generic Iwahori-Hecke algebras.
\end{abstract}

The imaginary cone ([33, Ch 5]) of a Kac-Moody Lie algebra is the convex hull of zero and the positive imaginary roots. The combinatorial characterization of imaginary roots from [33] can be used to give a definition ([29], 28]) of the imaginary cone which makes sense for possibly non-crystallographic root systems of Coxeter groups (which do not have imaginary roots in general).

This paper systematically studies the imaginary cone for a class of root systems of general Coxeter groups $W$. As well as in 33 for the crystallographic case, some of the basic facts may be found in 28, 22, 25] and 30. One of the main new results (Theorem 6.3) is that the imaginary cone of a reflection subgroup $W^{\prime}$ of $W$ is contained in the imaginary cone of $W$; the corresponding result for the closures of the imaginary cones is easier to prove but much less useful. Another main result is that, for irreducible infinite $W$ of finite rank, the closed imaginary cone is the only non-zero closed pointed $W$-stable cone contained in the pointed cone spanned by the simple roots (Theorem 7.6). A third main result gives algebraic descriptions of the face lattices of the imaginary cone and Tits cone. Namely, it is shown in Section 11 that (under mild finiteness and non-degeneracy conditions) several notions of faces of the imaginary cone (and of the Tits cone) coincide, and that the face lattice of the imaginary cone is isomorphic to the lattice of special facial subgroups of $W$ (facial subgroups with no finite components), and is dual to the lattice of faces of the Tits cone. Here, the facial subgroups are defined as the (parabolic) reflection subgroups arising as stabilizers of points of the Tits cone. In the framework of standard crystallographic root systems of Kac-Moody Lie

2010 Mathematics Subject Classification. 20F55, 17B22. 
algebras, with linearly independent simple roots and simple coroots, corresponding results on the faces and the face lattice of the Tits cone (but not the imaginary cone) have been obtained in work of Looijenga, Slodowy and Mokler (35], 45], [46], 38, 40], 41]). Those results play a significant role in the study of the face monoid associated to a Kac-Moody group (see [39]).

The relation between the Tits and imaginary cones is quite delicate since, in general, neither is a closed cone and many of the analogous facts do not even hold for closed cones. Another subtlety in the relationships between imaginary cones of reflection subgroups is that, even if all parabolic subgroups of $W$ are facial, not all parabolic subgroups of (finite rank) reflection subgroups $W^{\prime}$ of $W$ are facial; these relationships have not been known in the Kac-Moody case where, due to the nature of standard realizations of generalized Cartan matrices usually used, work has been mostly restricted to the special case where parabolic and facial subgroups coincide.

The results involving relationships between the imaginary cone of $W$ and its dihedral reflection subgroups have applications, to be given in subsequent papers, to the study of dominance order on root systems of general Coxeter groups, and certain associated finite state automata. Some of these have also been obtained in [22, 25] or 24. The results have connections with generic Iwahori-Hecke algebras; they lead to a construction of modules for certain Iwahori-Hecke algebras which provide a proof for finite rank Coxeter systems of a weakened version of a conjecture of Lusztig on boundedness of the $a$-function (recent work of Nanhua Xi 52 provides a proof of Lusztig's conjecture itself in the special case of Coxeter groups with complete Coxeter graphs).

In some detail, the contents of the paper are as follows. Section 1 gives basic properties of the class of reflection representations and root systems of general Coxeter groups which are used in this paper. It is obviously necessary to use a class of root systems closed under passage to subsystems of reflection subgroups, for which simple roots need not be linearly independent. We take for simplicity a minimal natural class of root systems with this property, in real vector spaces $V$ equipped with a $W$-invariant symmetric bilinear form.

Several of the basic results of this paper extend to more general classes of possibly non-symmetrizable root systems considered in [16, [15, and [26, which include also the standard crystallographic root systems of Kac-Moody Lie algebras. Although it would be desirable to give these extensions, as suggested by the above comments on what is known in the Kac-Moody setting, we do not go into this. The extension of results involving isotropic vectors, totally isotropic faces of the imaginary cone etc presents particular difficulties and should be especially interesting.

Section 2 records basic properties of facial subgroups (the stabilizers in $W$ of points of the Tits cone). It would be interesting to develop a suitable theory of root systems and corresponding facial subgroups, as in Section 2 in (possibly infinite and even infinite rank) oriented matroids and to determine to what extent results proved here hold in such an abstract setting.

Section 3 gives several equivalent definitions of the imaginary cone $\mathscr{Z}=\mathscr{Z}_{W}$ of $W$. The one corresponding most closely to the original definition in the Kac-Moody setting is as follows: $\mathscr{Z}_{W}=W \mathscr{K}_{W}$ where $\mathscr{K}_{W}$ is the intersection of the negative of the fundamental chamber with the cone $\mathbb{R}_{\geq 0} \Pi$ of non-negative linear combinations of simple roots $\Pi$ of $W$. In particular, $\mathscr{Z}$ is contained in the negative of the Tits cone (which is defined as the union of $W$-orbits of points of the fundamental 
chamber), and so the set $\mathscr{K}_{W}$ is a fundamental domain for $W$ acting on $\mathscr{Z}$. Also, $\mathscr{Z} \subseteq \mathbb{R}_{>0} \Pi$. A fact proved in Section 3 which is important for later developments is that the imaginary cone of a facial reflection subgroup $W^{\prime}$ is the intersection of the imaginary cone of $W$ with the subspace spanned by the roots of $W^{\prime}$.

In Sections 4 11, additional finiteness and non-degeneracy assumptions are imposed on the root system; in particular, $W$ is of finite rank throughout these sections. These conditions and their consequences are discussed in Section 4.

Section 5 establishes some facts, originally due to Kac (in the Kac-Moody setting), about the closure of the imaginary cone. In particular, it is shown that the closed imaginary cone is dual to the closure of the Tits cone, and it is the convex hull of the union of zero and all the limit rays of rays spanned by positive roots. The structure of the set of these limit rays has also been investigated in [30]. We state without proof here an important characterization of these limit rays from [30]: namely, the set of limit rays of positive roots is the closure of the union of the sets of limit rays of positive roots of dihedral reflection subgroups.

Section 6 proves the first main new result of this paper, Theorem 6.3, which asserts that $\mathscr{Z}_{W^{\prime}} \subseteq \mathscr{Z}$ if $W^{\prime}$ is a reflection subgroup of $W$. An important corollary is that if $W^{\prime \prime}$ is a facial reflection subgroup of $W$, then $\mathscr{Z}_{W^{\prime}} \cap \mathscr{Z}_{W^{\prime \prime}}=\mathscr{Z}_{W^{\prime \prime \prime}}$ where $W^{\prime \prime \prime}=W^{\prime} \cap W^{\prime \prime}$ (which is a facial reflection subgroup of $W^{\prime}$ by Section 2 ).

Section 7 gives our second main new result, Theorem 7.6, which asserts that for irreducible infinite finite rank $W$, the closed imaginary cone is the only nonzero pointed closed $W$-invariant cone contained in the pointed cone spanned by the simple roots. It is proved by showing that the limit rays of a $W$-orbit of rays in the imaginary cone include the limit rays of all dihedral reflection subgroups and invoking the above-mentioned result of [30]. The result has additional significant applications to the study of the $W$-action on the closed imaginary cone which are deferred to [21. In particular, Theorem 7.6 opens the way for the study of fractal properties of the imaginary cone and the dynamics of the $W$-action on it.

For an element $v$ of $\mathbb{R}_{>0} \Pi$, a support of $v$ is defined to be a subset $\Delta$ of $\Pi$ such that $v=\sum_{\alpha \in \Delta} c_{\alpha} \alpha$ with all $c_{\alpha}>0$. This notion of support is more subtle than in the case of linearly independent $\Pi$, when each $v$ has a unique support, and plays an important technical role throughout this paper. Section 8 collects some basic properties of supports additional to those already used in previous sections.

Section 9 discusses the closed imaginary cones of hyperbolic groups $W$ or hyperbolic reflection subgroups $W^{\prime}$ of (possibly non-hyperbolic) Coxeter groups $W$, and, as an extended example, the imaginary cones of "generic" universal root systems (those for which any rank two parabolic root subsystem is infinite and not affine). These classes of root systems play an important role in relation to general root systems. The main result obtained in the generic universal case is that (in rank at least three) the space of components of the set of points of the closed imaginary cone outside the imaginary cone is a Cantor space (the components themselves are totally isotropic cones of unknown dimensions and structure). This section also raises some natural questions left open in this work; others are deferred to [21.

In preparation for the main results on facial structure of the imaginary and Tits cones, the reader should consult the Appendix A which discusses the general algebraic aspects of facial structure of cones and introduces the (partly non-standard) terminology we use. It also collects for the reader's convenience at the end a few 
additional facts used extensively throughout this paper. A semidual pair of (possibly non-closed) cones is defined to be a pair of cones, each contained in the dual of the other. There are several notions of face (of each cone in the pair) which are applicable in such a context; (non-empty) extreme subsets, exposed (equivalently, semi-exposed) subsets, and stable sets for the natural Galois connection, corresponding to the relation of orthogonality of their elements, between subsets of the two cones. For trivial reasons, the second and third notions don't necessarily coincide for non-closed cones while, as is well known, the first and second notions don't necessarily agree even for a pair of mutually dual closed cones. We say the semidual pair is a dual pair of cones if all three notions of face of each cone in the pair coincide and the closures of the two cones are mutually dual in the usual sense. Polyhedral cones and their duals provide examples of dual pairs.

Section 10 is devoted to a detailed discussion of the facial structure of the imaginary cone and Tits cone. The main result (Theorem 10.3) on the face lattices obtained here is that the imaginary cone and Tits cone form a dual pair as discussed above, so that there are natural notions of face lattice of each, and that the face lattice of $\mathscr{Z}$ (and therefore the dual of the face lattice of the Tits cone) is canonically isomorphic to the lattice of special facial reflection subgroups of $W$.

The facial closure of a subset of $W$ is defined to be the unique inclusion-minimal facial subgroup containing it. Section 11 gives two algorithms for computing the facial closure of finitely generated subgroups of $W$. The first applies only to reflection subgroups, and determines the facial closure by means of geometry of the imaginary cone and root system, while the second applies to any finitely-generated subgroup and makes use of the solvability of the conjugacy problem for $W$.

Section 12 drops the finiteness and non-degeneracy assumptions 4.1(i)-(iii) and returns to the more general framework of Sections 1 1 3 . Under these relaxed assumptions, some of the statements about $\mathscr{Z}$ proved in Sections 411 would need to be modified if they are to hold, and we do not go into this. Three complicating factors are the choice of topology on the ambient real vector space, the appropriate generalization of the notion of dual pair of cones and the fact that, for infinite rank Coxeter systems, arbitrary intersections of facial subgroups need not be facial (see 42 for the analogous fact for parabolic subgroups). However, the main result of Section 6 is extended by showing that the imaginary cone of a reflection subgroup $W^{\prime}$ of $W$ is contained in that of $W$ in general.

The main part of this work was done in the academic year 2008-2009 while on sabbatical from the University of Notre Dame at the University of Sydney, though the results of Section 7, 2.13 2.17 and 9.9 9.18 were obtained later. I gratefully acknowledge the support of both institutions. I also thank Bob Howlett and Xiang $\mathrm{Fu}$ for some useful conversations and Claus Mokler for helpful communications. Finally, I thank the authors of [30] for useful conversations and for permission to use results in a preliminary version of 30 here.

\section{Recollections on Coxeter groups and Root systems}

This section fixes some commonly used notation and terminology, and describes basic properties of Coxeter groups and their root systems.

1.1. It is assumed that the reader is familiar with basic results on convexity, especially properties of polyhedral cones, their faces, extreme rays, dual polyhedral cones etc as described in [8] and [1] for instance. A detailed discussion of some 
aspects of convexity which are particularly relevant to the latter parts of this paper, especially notions of facial structure of general cones, is given in Appendix A This does not depend on other sections of this paper and can be consulted for more background and terminology on cones and convexity than given in this subsection.

Vector spaces are regarded as affine spaces, and affine spaces regarded as vector spaces by a choice of an origin, in the standard way. Finite dimensional vector spaces are always endowed with their standard topology. Except in Appendix A an infinite dimensional real vector space is always given a fixed but arbitrary topology coherent with the standard topologies on its finite dimensional subspaces.

Let $V$ be a real vector space. A subset of $V$ which is closed under multiplication by non-zero scalars is called a possibly non-convex cone in $V$. A cone in $V$ is a possibly non-convex cone which is convex as a subset of $V$. Equivalently, it is an additive subsemigroup of $(V,+)$ which is closed under multiplication by positive scalars. The zero cone $\{0\}$ is often written just as 0 and the empty cone as $\emptyset$. If $A \subseteq B \subseteq V$ with $A$ a cone, we say that $A$ is a conical subset of $B$ (or a subcone of $B$, if $B$ is a cone). A $x A .11$ of a possibly non-convex cone $A$ is a subset $B$ of $A \backslash\{0\}$ such that the map $(\lambda, v) \mapsto \lambda v: \mathbb{R}_{>0} \times B \rightarrow A \backslash\{0\}$ is bijective. Every possibly non-convex cone has a base, but a cone need not necessarily have a convex base. A (possibly non-convex) cone $\mathscr{Y}$ is said to be pointed if $0 \in \mathscr{Y}$ and blunt if $0 \notin Y$.

For subsets $A, B$ of $V$, define $A+B, A-B$ by $A \pm B=\{a \pm b \mid a \in A, b \in B\}$ and set $\lambda A:=\{\lambda a \mid a \in A\}$ for $\lambda \in \mathbb{R}$. The linear (resp., affine, convex or conical) span of $A$ is defined to be the smallest vector subspace (resp., affine set, convex set, cone) in $V$ which contains $A$ and is denoted $\operatorname{lin}(A)$ (resp., $\operatorname{aff}(A), \operatorname{conv}(A)$, cone $(A)$ ). These sets have well-known descriptions in terms of linear (resp., affine, convex, positive linear) combinations of elements of $A$. One has cone $(A)=\{\lambda v \mid$ $\left.v \in \operatorname{conv}(A), \lambda \in \mathbb{R}_{>0}\right\}$ and $\operatorname{aff}(A)=A+\operatorname{cone}(A-A)$. If $A \neq \emptyset$, then $\operatorname{aff}(A)=$ $a+\operatorname{cone}(A-A)$ for any $a \in A$ and aff $A$ then has a natural structure of affine space. One has $\operatorname{lin}(A)=\operatorname{aff}(A \cup\{0\})=\operatorname{cone}(A \cup\{0\} \cup-A)$ where $-A:=(-1) A$. If $A$ is a non-empty cone, then $\operatorname{lin}(A)=\operatorname{aff}(A)$. We abbreviate $\mathbb{R} A:=\operatorname{lin}(A)$ and $\mathbb{R}_{\geq 0} A:=\operatorname{cone}(A \cup\{0\})$. Define $\mathbb{R}_{>0} A:=\left\{\sum_{a \in A} c_{a} a \mid c_{a} \in \mathbb{R}_{>0}\right.$ for all $\left.a \in A\right\}$ if $A$ is finite; in particular, $\mathbb{R}_{>0} \emptyset=\{0\}$ and $\mathbb{R}_{>0} \emptyset=\emptyset$. We say that $\Gamma \subseteq V$ is positively independent if $0 \notin \mathbb{R}_{>0} A$ for any finite $A \subseteq \Gamma$. Frequently, we do not distinguish notationally between a singleton $\{v\}$ and $v$, writing for example $A-v$ for $A-\{v\}$ and $A \backslash v$ for $A \backslash\{v\}$ (where $A \backslash B$ denotes set difference).

A cone $\mathscr{Y}$ is said to be salient if $\mathscr{Y} \cap-\mathscr{Y}=\{0\}$ and generating if $\mathscr{Y}-\mathscr{Y}=V$. A ray of $V$ is a cone of the form $\mathbb{R}_{\geq 0} \alpha$ for some non-zero $\alpha \in V$; we say that $\alpha$ spans, or generates, the ray $\mathbb{R}_{\geq 0} \alpha$.

Assume $V$ is topologized as described above. The closure of a subset $A$ of $V$ is denoted as $\bar{A}$ or $\operatorname{cl}(A)$. The interior of $A$ is denoted $\operatorname{int}(A)$. The relative interior, denoted $\operatorname{ri}(A)$ or sometimes $A^{\circ}$, of a subset $A$ of $V$ is defined to be the interior of $A$ relative to the subspace topology of $\operatorname{aff}(A)$. The relative boundary $\operatorname{rb}(A)$ of $A$ is defined by $\operatorname{rb}(A):=\operatorname{cl}(A) \backslash \operatorname{ri}(A)$. The set of limit points of a set or sequence $X$ in a topological space is denoted $\operatorname{Acc}(X)$. Recall that any non-empty convex set (e.g. a non-empty cone) is connected.

1.2. Consider a real vector space $V$ equipped with a symmetric $\mathbb{R}$-bilinear form $\langle-,-\rangle: V \times V \rightarrow \mathbb{R}$. We call the pair $(V,\langle-,-\rangle)$ a quadratic space (over $\mathbb{R}$ ). In general, for $A, B \subseteq V$, write $\langle A, B\rangle=\{\langle a, b\rangle \mid a \in A, b \in B\}$. Define $A^{\perp}:=\{v \in V \mid\langle v, A\rangle \subseteq\{0\}\}$ and $A^{*}:=\left\{v \in V \mid\langle v, A\rangle \subseteq \mathbb{R}_{\geq 0}\right\}$. Write $A \perp B$ 
if $B \subseteq A^{\perp}$. It is well-known (see Appendix A more generally) that $A^{*}$ is a pointed cone and that if $V$ is finite dimensional and $\langle-,-\rangle$ is non-singular, then $A^{*}$ is closed and $A^{* *}=\operatorname{cl}\left(\mathbb{R}_{\geq 0} A\right)$. The quadratic space (or form) is said to be non-singular if $V^{\perp}=0$. If $V$ is finite-dimensional, we say that the form (or quadratic space) $\langle-,-\rangle$ is of signature $(k, l, m)$ if a matrix of the form has $k$ positive eigenvalues, $l$ negative eigenvalues and $m$ zero eigenvalues.

A vector $\alpha \in V$ is said to be isotropic (resp., non-isotropic) if $\langle\alpha, \alpha\rangle=0$ (resp., $\langle\alpha, \alpha\rangle \neq 0$ ). If $\alpha \neq 0$, the ray $\mathbb{R}_{\geq 0} \alpha$ spanned by $\alpha$ will be said to be positive, negative or isotropic according as $\langle\alpha, \alpha\rangle$ is positive, negative or zero. Let $\mathscr{Q}:=\{\alpha \in V \mid\langle\alpha, \alpha\rangle=0\}$ denote the possibly non-convex cone of isotropic vectors in $V$.

For non-isotropic $\alpha \in V$, let $s_{\alpha}: V \rightarrow V$ denote the reflection in $\alpha$, given by

$$
s_{\alpha}(v):=v-\left\langle v, \alpha^{\vee}\right\rangle \alpha, \quad \alpha^{\vee}:=\frac{2}{\langle\alpha, \alpha\rangle} \alpha .
$$

For any $\Gamma \subseteq V$ consisting of non-isotropic vectors, let $W_{\Gamma}:=\left\langle s_{\gamma} \mid \gamma \in \Gamma\right\rangle$ denote the subgroup of $\mathrm{GL}(V)$ generated by reflections in elements of $\Gamma$.

1.3. Assume given a based root system $(\Phi, \Pi)$ for $(V,\langle-,-\rangle))$ with associated Coxeter system $(W, S)$ in the sense of [3, §3]. By definition, this means that

(i) $(V,\langle-,-\rangle)$ is a quadratic space (over real).

(ii) $\Pi \subseteq V$ is positively independent.

(iii) $\langle\alpha, \alpha\rangle=1$ for $\alpha \in \Pi$. Set $m_{\alpha, \alpha}:=1$ for all $\alpha \in \Pi$.

(iv) For $\alpha \neq \beta \in \Pi$, either $\langle\alpha, \beta\rangle=-\cos \frac{\pi}{m_{\alpha, \beta}}$ where $m_{\alpha, \beta} \in \mathbb{N}_{\geq 2}$ or $\langle\alpha, \alpha\rangle \leq$ -1 , in which case we set $m_{\alpha, \beta}:=\infty$.

(v) $S=\left\{s_{\alpha} \mid \alpha \in \Pi\right\}$ and $W=W_{\Pi}$ is the group of $\mathbb{R}$-linear automorphisms of $V$ generated by $S$.

(vi) $\Phi:=\{w \alpha \mid w \in W, \alpha \in \Pi\}$.

The pair $(W, S)$ is a Coxeter system with Coxeter matrix $\left(m_{\alpha, \beta}\right)_{\alpha, \beta \in \Pi}$; in particular, for $\alpha, \beta \in \Pi, s_{\alpha} s_{\beta}$ has order $m_{\alpha, \beta}$. Any Coxeter system is isomorphic to one attached to a "standard" based root system as above, in which $\Pi$ is a basis for $V$ and $\langle\alpha, \beta\rangle \geq-1$ for all $\alpha, \beta \in \Pi$ (see [32]). It also isomorphic to one attached to a based root system in which $\Pi$ is linearly independent and $\langle-,-\rangle$ is non-singular. Coxeter systems in this paper are possibly of infinite rank except where otherwise stated (as by blanket assumption in Sections 4 11). The notion of subgroup always refers to a subgroup of $W$ unless the overgroup is more precisely indicated.

One calls $\Pi$ the set of simple roots and $\Phi$ the root system. The set $\Phi_{+}:=\Phi \cap \mathbb{R}_{\geq 0} \Pi$ is called the set of positive roots. We have $\Phi=\Phi_{+} \cup \dot{U} \Phi_{-}$, where $\Phi_{-}:=-\Phi_{+}$and $\dot{U}$ indicates that a union is of disjoint sets. The cone $\mathbb{R}_{>0} \Pi=\mathbb{R}_{>0} \Phi_{+}$will be called the positive root cone. Denote the standard length function of $(W, S)$ as $l: W \rightarrow \mathbb{N}$ and define the set $T:=\left\{w s w^{-1} \mid w \in W, s \in S\right\}$ of reflections. The map $\alpha \mapsto s_{\alpha}: \Phi_{+} \rightarrow T$ is a bijection. For $t \in T$, we sometimes denote the unique positive root $\alpha$ with $s_{\alpha}=t$ as $\alpha_{t}$.

For standard properties of Coxeter systems and root systems, see [4], 32] and [2]. Frequently, results in these and other references are stated under stronger conditions than imposed in this paper (e.g. with $\Pi$ finite, or for the standard root system), but the proofs of our stated results under the weaker conditions here are essentially the same unless otherwise indicated. Useful properties of the based root systems considered in this paper are listed in [3. The conditions (i)-(iv) imply that for any 
finite subset $\Delta$ of $\Pi$, the pointed conical hull $C:=\mathbb{R}_{\geq 0} \Delta$ of $\Delta$ is a polyhedral cone and the extreme rays of $C$ are spanned by the elements of $\Delta$; hence there is a linear map $\rho: V \rightarrow \mathbb{R}$ with $\rho(\Delta) \subseteq \mathbb{R}_{>0}$. Therefore if $\Pi$ is finite, then $(V,\langle\cdot \mid \cdot\rangle, \Pi)$ is a root basis in the sense of [34] and the results of [34] and [31] apply.

Remarks. (1) Suppose given a based root system $(\Phi, \Pi)$ in $(V,\langle-,-\rangle)$. For any subspace $V^{\prime} \supseteq \mathbb{R} \Pi$ of $V$, we may regard $(\Phi, \Pi)$ as a based root system in $\left(V^{\prime},\langle-,-\rangle^{\prime}\right)$ where $\langle-,-\rangle^{\prime}$ is obtained by restriction of $\langle-,-\rangle$ to a symmetric bilinear form on $V^{\prime}$; the latter based root system is said to be obtained by restriction (of quadratic space) of the former, and the former by extension (of quadratic space) of the latter. The associated Coxeter systems of based root systems related by extension or restriction are canonically isomorphic.

(2) A based root system $(\Phi, \Pi)$ in $(V,\langle-,-\rangle)$ will be said to be ample for a subspace $U$ of $V$ if any $\mathbb{R}$-linear map $U \rightarrow \mathbb{R}$ is of the form $u \mapsto\langle u, v\rangle$, for $u \in V$, for some $v \in V$; this holds for instance if $U$ is finite-dimensional and $\langle-,-\rangle$ is non-singular. In case $U=\mathbb{R} \Pi$, we say simply that the based root system is ample or that $(V,\langle-,-\rangle)$ is ample for $(\Phi, \Pi)$. It is not difficult to show that for any based root system $(\Phi, \Pi)$ in $(V,\langle-,-\rangle)$ and any subspace $U$ of $V$, there is an extension which is ample for $U$ and such that the associated quadratic space is non-singular.

1.4. For proofs of some (but not all) facts concerning $(W, S)$ or $\Phi$, it is possible to replace the root system by one with linearly independent roots by a standard construction used for instance in the proof of [31, Proposition 2.9] and [13, (3.5)]. We recall this construction below.

Let $V^{\prime}$ be a real vector space with linearly independent subset $\Pi^{\prime}=\left\{\alpha^{\prime} \mid \alpha \in \Pi\right\}$ in bijection with $\Pi$ by a map $\alpha^{\prime} \mapsto \alpha: \Pi^{\prime} \cong \Pi$. Suppose given a linear map $L: V^{\prime} \rightarrow V$ which restricts to this bijection $\Pi^{\prime} \rightarrow \Pi$ (if $\Pi^{\prime}$ is a basis for $V^{\prime}$, there is a unique such map $L^{\prime}$ ). Define a symmetric bilinear form $\langle-,-\rangle^{\prime}: V^{\prime} \times V^{\prime} \rightarrow \mathbb{R}$ by $\left\langle u_{1}, u_{2}\right\rangle^{\prime}=\left\langle L\left(u_{1}\right), L\left(u_{2}\right)\right\rangle$. For non-isotropic $u \in V^{\prime}$, let $r_{u}: V^{\prime} \rightarrow V^{\prime}$ denote the reflection in $u$. Let $W^{\prime}:=\left\langle S^{\prime}\right\rangle \subseteq \operatorname{GL}\left(V^{\prime}\right)$ where $S^{\prime}:=\left\{r_{\alpha} \mid \alpha \in \Pi^{\prime}\right\} \subseteq \operatorname{GL}\left(V^{\prime}\right)$, $\Phi^{\prime}:=W^{\prime} \Pi^{\prime}$ and $\Phi_{+}^{\prime}=\Phi^{\prime} \cap \mathbb{R}_{>0} \Pi^{\prime}$. Then $\left(\Phi^{\prime}, \Pi^{\prime}\right)$ is a based root system for $\left(V^{\prime},\langle-,-\rangle^{\prime}\right)$ with associated Coxeter system $\left(W^{\prime}, S^{\prime}\right)$ and the map $r_{\alpha^{\prime}} \mapsto s_{\alpha}: S^{\prime} \rightarrow$ $S$ for $\alpha \in \Pi$ extends to an isomorphism of Coxeter systems $\theta:\left(W^{\prime}, S^{\prime}\right) \rightarrow(W, S)$. Further, $L$ restricts to bijections $\Phi^{\prime} \stackrel{\cong}{\rightarrow} \Phi$ and $\Phi_{+}^{\prime} \stackrel{\cong}{\rightarrow} \Phi_{+}$and satisfies $L(w u)=$ $\theta(w) L(u)$ for all $w \in W^{\prime}$ and $u \in V^{\prime}$ (i.e. identifying $W^{\prime}$ with $W$ by $\theta, L$ is a $W$-equivariant map).

If $\Pi^{\prime}$ is a basis of $V^{\prime}$, we shall call $\left(\Phi^{\prime}, \Pi^{\prime}\right)$ on $\left(V^{\prime},\langle-,-\rangle^{\prime}\right)$ a canonical lift of $(\Phi, \Pi)$ on $(V,\langle-,-\rangle)$ and call $L$ the associated canonical map. This notion will be used several times in this paper to reduce proofs to the case of linearly independent simple roots (i.e from the positive root cone being a general polyhedral cone to a simplicial cone). It seems likely that these arguments could be replaced by arguments using more of the theory of polyhedral cones.

The diversity of root bases which may be possible for fixed $(W, S)$ is suggested by the following example.

Example. Suppose $(W, S)$ is universal (i.e there are no braid relations) and of rank $n \geq 3$. We consider certain based root systems $(\Phi, \Pi)$ on spaces $(V,\langle-,-\rangle)$ with $V=\mathbb{R} \Pi$ and with associated Coxeter system isomorphic to $(W, S)$. The requirement that $W$ is universal is equivalent to the condition $\langle\alpha, \beta\rangle \leq-1$ for 
all distinct $\alpha, \beta \in \Pi$. Write $\Pi=\left\{\alpha_{1}, \ldots, \alpha_{n}\right\}$ and assume first that $\Pi$ is linearly independent. The standard based root system from [4] or [32] has $\left\langle\alpha_{i}, \alpha_{j}\right\rangle=-1$ for $i \neq j$. There is also a based root system $(\Phi, \Pi)$ on $(V,\langle-,-\rangle)$ in which $\Pi$ is linearly independent and $\left\langle\alpha_{i}, \alpha_{j}\right\rangle:=1-8(i-j)^{2}$ for all $i, j$. (The latter based root system arises naturally as a canonical lift of a subsystem of a based root system considered in Example 1.7). It is obvious from its natural origin (and easy to check directly) that the latter form on $V$ has signature $(2,1, n-3)$. By perturbing $\langle-,-\rangle$ on $V$ and dividing out by a subspace of the radical (taking the image of $\Pi$ in the quotient space as new simple roots, see [34, 6.1]) one can find based root systems, $\left(\Phi^{\prime}, \Pi^{\prime}\right)$ on $\left(V^{\prime},\langle-,-\rangle^{\prime}\right)$, of $(W, S)$ such that the form $\langle-,-\rangle^{\prime}$ on $\mathbb{R} \Pi^{\prime}$ is of any signature $(k, l, m)$ with $k+l+m \leq n, k \geq 2$ and $l \geq 1$ and with the positive root cone $\mathbb{R}_{\geq 0} \Pi^{\prime}$ of any of a wide variety of combinatorial types of $k+l+m$-dimensional cones with $n$ extreme rays. It is easily checked that this is the full range of possible signatures (for the bilinear form on the span of the roots) for irreducible, infinite non-affine root systems.

Remarks. Despite the diversity of based root systems, their root systems $\Phi$ (for fixed $W$ ) are all canonically isomorphic as $W \times\{ \pm 1\}$-sets. For the bijection $(t, \epsilon) \mapsto$ $\epsilon \alpha_{t}: T \times\{ \pm 1\} \rightarrow \Phi$ may be used to transfer the $W \times\{ \pm 1\}$-action on $\Phi$ to one on $T \times\{ \pm 1\}$; the resulting action depends only on $(W, S)$ as Coxeter system (it is described in [4, Ch IV, $\S 1$, no. 4]).

1.5. It is well known (see 32, for example) that for $w \in W$,

$$
N(w):=\{t \in T \mid l(t w)<l(w)\}=\left\{s_{\alpha} \mid \alpha \in \Phi_{+} \cap w\left(-\Phi_{+}\right)\right\} .
$$

The equality above will be used frequently without special comment. Any reflection subgroup $W^{\prime}$ of $W$ (i.e. a subgroup $W^{\prime}=\left\langle W^{\prime} \cap T\right\rangle$ generated by the reflections it contains) has a canonical set of Coxeter generators $\chi\left(W^{\prime}\right)=\left\{t \in T \mid N(t) \cap W^{\prime}=\right.$ $\{t\}\}([17,3.3])$. We always consider reflection subgroups as Coxeter groups with these Coxeter generators, unless otherwise stated. It is easily shown (see [31, Lemma 2.8]) that if $\Gamma, \Gamma^{\prime} \subseteq \Phi$ with $W_{\Gamma}=W_{\Gamma^{\prime}}$, then $\mathbb{R} \Gamma=\mathbb{R} \Gamma^{\prime}$.

A reflection subgroup $W^{\prime}$ is said to be dihedral if it is generated by two (distinct) reflections, or equivalently (by [17, 3.11]) if $\left|\chi\left(W^{\prime}\right)\right|=2$. Any dihedral reflection subgroup $\left\langle s_{\alpha}, s_{\beta}\right\rangle$ is contained in a unique maximal (under inclusion) dihedral reflection subgroup, namely $\left\langle s_{\gamma} \mid \gamma \in \Phi \cap(\mathbb{R} \alpha+\mathbb{R} \beta)\right\rangle$ (see [19, 3.2]).

1.6. For any reflection subgroup $W^{\prime}$ and any $w \in W$, the coset $W^{\prime} w$ has a unique element $x$ of minimal length, characterized by $x \in W^{\prime} w$ and $N(x) \cap\left(W^{\prime} \cap T\right)=\emptyset$ or equivalently, $x \in W^{\prime} w$ and $N(x) \cap \chi\left(W^{\prime}\right)=\emptyset([17,3.4]$, [19, 1.4]), or equivalently again, $x \in W^{\prime} w$ and $x^{-1}\left(\Pi_{W^{\prime}}\right) \subseteq \Phi_{+}$. One has $\chi\left(w^{-1} W^{\prime} w\right)=x^{-1} \chi\left(W^{\prime}\right) x$ by [20, Lemma 1].

1.7. For any reflection subgroup $W^{\prime}$ of $(W, S)$, let

$$
\Phi_{W^{\prime}}:=\left\{\alpha \in \Phi \mid s_{\alpha} \in W^{\prime}\right\}, \quad \Phi_{W^{\prime},+}:=\Phi_{W^{\prime}} \cap \Phi_{+}
$$

denote the corresponding sets of roots and positive roots, and

$$
\Pi_{W^{\prime}}:=\left\{\alpha \in \Phi_{+} \mid s_{\alpha} \in \chi\left(W^{\prime}\right)\right\} .
$$

Then $\left(\Phi_{W^{\prime}}, \Pi_{W^{\prime}}\right)$ is a based root system in $V$ with positive roots $\Phi_{W^{\prime},+}$ and associated Coxeter system $\left(W^{\prime}, \chi\left(W^{\prime}\right)\right)\left(\left[3\right.\right.$, Lemma 3.5]). From [17, a subset $\Gamma$ of $\Phi_{+}$is 
of the form $\Gamma=\Pi_{W^{\prime}}$ for some reflection subgroup $W^{\prime}$ of $W$ (necessarily $W^{\prime}=W_{\Gamma}$ ) if and only if $\langle\alpha, \beta\rangle \in(-\infty,-1] \cup\left\{-\cos \frac{\pi}{n} \mid n \in \mathbb{N}_{>2}\right\}$ for all distinct $\alpha, \beta \in \Pi$.

If $w \in W$ with $N(w) \cap W^{\prime}=\emptyset$ then using 1.51 .6 and the definitions, one has

$$
\Pi_{w^{-1} W^{\prime} w}=w^{-1} \Pi_{W^{\prime}}, \quad \Phi_{w^{-1} W^{\prime} w}=w^{-1} \Phi_{W^{\prime}}, \quad \Phi_{w^{-1} W^{\prime} w,+}=w^{-1} \Phi_{W^{\prime},+} .
$$

Example. (cf. 13, Example 3.19]) Let $(\Phi, \Pi)$ on $(V,\langle-,-\rangle)$ be the standard based root system of a rank 3 universal Coxeter group, with $\Pi=\{\alpha, \beta, \gamma\}$ as $\mathbb{R}$-basis for $V$ and $\langle\delta, \delta\rangle=-\langle\delta, \epsilon\rangle=1$ for all $\delta \neq \epsilon$ in $\Pi$. For $k \in \mathbb{Z}$, define $\gamma_{k}=2 k(2 k+1) \alpha+2 k(2 k-1) \beta+\gamma$. A simple computation shows that $s_{\alpha} s_{\beta}\left(\gamma_{k}\right)=$ $\gamma_{k+1}$. Since $\gamma_{0}=\gamma$, it follows that $\gamma_{k}=\left(s_{\alpha} s_{\beta}\right)^{k}(\gamma)$ for all $k \in \mathbb{Z}$. We have $\left\langle\gamma_{i}, \gamma_{j}\right\rangle=\left\langle\gamma, \gamma_{j-i}\right\rangle=1-8(i-j)^{2}$ for all $i, j \in \mathbb{Z}$. Setting $W^{\prime}:=\left\langle s_{\gamma_{k}} \mid k \in \mathbb{Z}\right\rangle$, it follows that $\Pi_{W^{\prime}}=\left\{\gamma_{k} \mid k \in \mathbb{Z}\right\}$. In particular, even though $(\Phi, \Pi)$ on $(V,\langle-,-\rangle)$ is a standard based root system, of finite rank, the based root system $\left(\Phi_{W^{\prime}}, \Pi_{W^{\prime}}\right)$ on $(V,\langle-,-\rangle)$ is of infinite rank and is not standard; $\Pi_{W^{\prime}}$ is linearly dependent and $\langle\delta, \epsilon\rangle<-1$ for distinct $\delta, \epsilon \in \Pi_{W^{\prime}}$. This phenomenon provides the principal reason for using the class of root systems in 1.3 rather than the standard root systems as in 32. Infinite rank reflection subgroups appear quite naturally; for example, even in finite rank root systems, the stabilizer of a root $\alpha$ is a semidirect product of its (sometimes infinite rank) normal reflection subgroup generated by reflections in all roots orthogonal to $\alpha$, and a free group.

1.8. In general, for any subset $I$ of $S$, let $W_{I}$ denote the subgroup of $W$ generated by $I$. Let $I^{\perp}:=\{r \in S \backslash I \mid s r=r s$ for all $s \in I\}$. The subgroups $W_{I}$ for $I \subseteq S$ are called standard parabolic subgroups of $W$, and their conjugates in $W$ are called parabolic subgroups of $W$. One has $\chi\left(W_{I}\right)=I$. Following [35, we shall say that $I \subseteq S$ (resp., $W_{I}, w W_{I} w^{-1}$ ) is a special subset of $S$ (resp., special standard parabolic subgroup, special parabolic subgroup) if $I$ has no component $J$ with $W_{J}$ finite. For any $I \subseteq S$, abbreviate $\Pi_{I}:=\Pi_{W_{I}}=\left\{\alpha \in \Pi \mid s_{\alpha} \in I\right\}$, $\Phi_{I}:=\Phi_{W_{I}}=W_{I} \Pi_{I}, \Phi_{I,+}=\Phi_{W_{I},+}$ etc. Denote the set of minimal length coset representatives of $W / W_{I}$ as $W^{I}$. Then

$$
W^{I}=\left\{x \in W \mid N\left(x^{-1}\right) \cap I=\emptyset\right\}=\left\{x \in W \mid x\left(\Pi_{I}\right) \subseteq \Phi_{+}\right\} .
$$

If $I, J, K \subseteq S$, let $W_{J}^{I}:=W_{J} \cap W^{I}$ and ${ }^{K} W_{J}^{I}:=W_{J}^{I} \cap\left(W^{K}\right)^{-1}$.

Remarks. We shall use at times standard properties of shortest double coset representatives which can be found for finite $W$ in $[9,2.7$ ] (they can be proved similarly for general $W$ ). In fact, many of these results can be generalized to apply to $\left(W^{\prime}, W_{J}\right)$ double cosets (where $W^{\prime}$ is an arbitrary reflection subgroup) and more general length functions than the standard ones, but we don't go into this here.

1.9. We shall now introduce several cones which play an important role in this paper. Let $W^{\prime}$ be a reflection subgroup of $W$. Let $\mathscr{C}_{W^{\prime}}$ denote the fundamental chamber for $W^{\prime}$ on $V$. By definition, this is the cone

$$
\begin{aligned}
\mathscr{C}_{W^{\prime}} & =\left\{v \in V \mid\langle v, \alpha\rangle \geq 0 \text { for all } \alpha \in \Pi_{W^{\prime}}\right\} \\
& =\left\{v \in V \mid\langle v, \alpha\rangle \geq 0 \text { for all } \alpha \in \Phi_{W^{\prime},+}\right\}
\end{aligned}
$$

Let $\mathscr{X}_{W^{\prime}}:=\cup_{w \in W^{\prime}} w\left(\mathscr{C}_{W^{\prime}}\right)$ denote the Tits cone (see 1.10 below) of $W^{\prime}$. Abbreviate $\mathscr{C}:=\mathscr{C}_{W}$ and $\mathscr{X}:=\mathscr{X}_{W}$.

Remarks. The fundamental chamber and Tits cone may change significantly under extension or restriction of quadratic space. Note that according to our definitions, 
$\mathscr{C} \cap-\mathscr{C}=\Pi^{\perp}$. This differs from other treatments (e.g. 4]) in which the fundamental chamber and Tits cone are defined as subsets of the dual space of the linear span $\mathbb{R} \Pi$ of the simple roots (so one has $\mathscr{C} \cap-\mathscr{C}=\{0\}$ ). If $\langle-,-\rangle$ is non-singular and $V$ is finite dimensional, then one may identify $V$ with its dual space and the definition is consistent with that in sources (e.g. [34, 31, 49, 33) where $\mathscr{C}$ is defined as a subset of the dual space of $V$. In general, if the quadratic space $(V,\langle-,-\rangle)$ is ample for $(\Phi, \Pi)$, then the dual space of $\mathbb{R} \Pi$ identifies with quotient subspace of $V$ by $\Pi^{\perp}$ and our notions have similar properties as in these other sources. In full generality, it is not obvious that $\mathscr{C}$ or $\mathscr{X}$ is non-zero, but we may always replace the based root system by an ample extension if that is inconvenient (see 12.1).

1.10. The following lemma records some basic properties of the Tits cone and fundamental chamber.

Lemma. (a) $\mathscr{X}$ is the set of all $v \in V$ which have negative inner product $\langle\alpha, v\rangle$ with only a finite number of positive roots i.e.

$$
\mathscr{X}_{W}=\left\{v \in V||\left\{\alpha \in \Phi_{+} \mid\langle v, \alpha\rangle<0\right\} \mid \text { is finite }\right\} .
$$

(b) $\mathscr{C}$ and $\mathscr{X}$ are pointed cones.

(c) For $x, y \in \mathscr{C}$ and $w \in W$, one has $w x=y$ if and only if $x=y$ and $w$ is in the standard parabolic subgroup generated by reflections in simple roots $\alpha$ with $\langle\alpha, x\rangle=0$.

(d) The stabilizer $\operatorname{Stab}_{W}(x)$ in $W$ of any point $x$ of $\mathscr{X}$ is generated by the reflections in the roots $\alpha$ with $\langle x, \alpha\rangle=0$, and is a parabolic subgroup.

(e) For a reflection subgroup $W^{\prime}$ of $W, \mathscr{C}_{W^{\prime}} \supseteq \mathscr{C}_{W}$ and $\mathscr{X}_{W^{\prime}} \supseteq \mathscr{X}_{W}$.

(f) If $W_{1}, \ldots, W_{n}$ are reflection subgroups of $W$ such that $\Phi=\cup_{i} \Phi_{W_{i}}$ then $\mathscr{C}_{W}=\cap_{i} \mathscr{C}_{W_{i}}$ and $\mathscr{X}_{W}=\cap_{i} \mathscr{X}_{W_{i}}$.

(g) For a reflection subgroup $W^{\prime}$ of $W$ and $w \in W, \mathscr{X}_{w W^{\prime} w^{-1}}=w\left(\mathscr{X}_{W^{\prime}}\right)$.

(h) If $W$ is finite, then $\mathscr{X}=V$.

Remarks. Part (f) applies if $W$ has finitely many components $W_{1}, \ldots, W_{n}$.

Proof. Parts (a)-(d) and (h) are standard (see [33, Ch 3], 4], 34]). Parts (e)-(f) are consequence of the definition of $\mathscr{C}$ and (a). If $w \in W^{\prime}$, (g) is trivial. By 1.6, it is therefore enough to check $(\mathrm{g})$ if $w$ is of minimal length in $w W^{\prime}$. In that case, $(\mathrm{g})$ follows using (a) and the fact from 1.7 that $\Phi_{w W^{\prime} w^{-1},+}=w\left(\Phi_{W^{\prime},+}\right)$.

1.11. The next lemma refines the relationship between fundamental chambers of reflection subgroups described in Lemma 1.10 (e).

Lemma. Let $W^{\prime}$ be a reflection subgroup of $W$ and let $W^{\prime \prime}$ be the set of minimal length coset representatives in $W^{\prime} \backslash W$. Then

(a) If $x \in W^{\prime \prime}$, then $\mathscr{C}_{x^{-1} W^{\prime} x}=x^{-1}\left(\mathscr{C}_{W^{\prime}}\right)$.

(b) $\mathscr{C}_{W^{\prime}} \cap \mathscr{X}_{W}=\bigcup_{w \in W^{\prime \prime}} w\left(\mathscr{C}_{W}\right)$.

Proof. Part (a) follows from the definition of $\mathscr{C}_{W^{\prime}}$, using $\Pi_{x^{-1} W^{\prime} x}=x^{-1}\left(\Pi_{W^{\prime}}\right)$ from 1.7. For (b), note first that if $w \in W^{\prime \prime}$, then (a) and Lemma 1.10(e) imply that $w^{-1}\left(\mathscr{C}_{W^{\prime}}\right)=\mathscr{C}_{w^{-1} W^{\prime} w} \supseteq \mathscr{C}_{W}$. Hence $\cup_{w \in W^{\prime \prime}} w\left(\mathscr{C}_{W}\right) \subseteq \mathscr{C}_{W^{\prime}} \cap \mathscr{X}_{W}$. To prove the reverse inclusion, let $v \in \mathscr{C}_{W^{\prime}} \cap \mathscr{X}_{W}$. Write $v=w\left(v^{\prime}\right)$ where $v^{\prime} \in \mathscr{C}_{W}$ and $w \in W$ is of minimal length $l(w)$. It will suffice to show that $l\left(s_{\alpha} w\right) \geq l(w)$ for all $\alpha \in \Pi_{W^{\prime}}$, for then $w \in W^{\prime \prime}$ by 1.6. Suppose first that $\langle\alpha, v\rangle=0$. Then $v=s_{\alpha}(v)=\left(s_{\alpha} w\right)\left(v^{\prime}\right)$ with $s_{\alpha} w \in W$. By choice of $w, l\left(s_{\alpha} w\right) \geq l(w)$. On the other hand, suppose 
$\langle\alpha, v\rangle \neq 0$. Since $v \in \mathscr{C}_{W^{\prime}}$, this forces $0<\langle v, \alpha\rangle=\left\langle w\left(v^{\prime}\right), \alpha\right\rangle=\left\langle v^{\prime}, w^{-1}(\alpha)\right\rangle$. Since $v^{\prime} \in \mathscr{C}_{W}$ and $w^{-1}(\alpha) \in \Phi$, it follows that $w^{-1}(\alpha) \in \Phi_{+}$and so $l\left(s_{\alpha} w\right)>l(w)$ as required.

1.12. The following result is in marked contrast to the case of finite $W$ (see $1.10(h))$.

Proposition. Suppose that $(W, S)$ is infinite, irreducible and not affine, and $S$ is finite. Let $V^{\prime}=\mathbb{R} \Pi$ be the subspace of $V$ spanned by $\Pi$ and $X:=\mathscr{X} \cap V^{\prime}$. Then $\bar{X} \cap-\bar{X}=\{0\}$.

Proof. See [31, Proposition 3.2], [34, Theorem 2.16] and [49, Lemma 15].

1.13. The next lemma records well-known useful facts about $W$-orbits on $V$

Lemma. (a) If $w=s_{\alpha_{1}} \cdots s_{\alpha_{n}}$, with all $\alpha_{i} \in \Phi$ then for $v \in V$,

$$
v-w(v)=\sum_{i=1}^{n}\left\langle v, \alpha_{i}^{\vee}\right\rangle \beta_{i}
$$

where $\beta_{i}:=s_{\alpha_{1}} \cdots s_{\alpha_{i-1}}\left(\alpha_{i}\right)$. Further, $w=s_{\beta_{n}} \ldots s_{\beta_{1}}$.

(b) If $w=s_{\alpha_{1}} \cdots s_{\alpha_{n}}$ is a reduced expression for $w$, with all $\alpha_{i} \in \Pi$, then

$\beta_{1}, \ldots, \beta_{n}$ are distinct positive roots, with $\Phi_{+} \cap w\left(-\Phi_{+}\right)=\left\{\beta_{1}, \ldots, \beta_{n}\right\}$.

(c) For any $w \in W$ and $v \in \mathscr{C}$, we have $v-w(v) \in \mathbb{R}_{\geq 0} \Pi$.

(d) If $W$ is irreducible and $v \in V$ satisfies $v \not \perp \Pi$, then aff $(W v)=v+\mathbb{R} \Pi$.

Proof. Parts (a) and (b) are readily proved by induction (see [33, Ch 3], 4, or 32]) and part (c) follows immediately from them. Part (d) is a variant of [4, Ch $5, \S 4$, Proposition 7]. Let $U$ be the translation space of aff $(W v)$, so $U=$ $\operatorname{lin}(\{x v-y v \mid x, y \in W\})=\operatorname{lin}(\{v-w v \mid w \in W\})$ and $\operatorname{aff}(W v)=v+U$. We have $U \subseteq \mathbb{R} \Pi$ by (c). Since $v \not \perp \Pi$, there is $\alpha \in \Pi$ with $\langle v, \alpha\rangle \neq 0$. Then $\alpha \in U$ since $\left\langle v, \alpha^{\vee}\right\rangle \alpha=v-s_{\alpha}(v)$. Further, if $\beta \in \Pi \cap U$ and $\gamma \in \Pi$ with $\langle\beta, \gamma\rangle \neq 0$, then $\gamma \in U$ since $\left\langle\beta, \gamma^{\vee}\right\rangle \gamma=\beta-s_{\gamma}(\beta)$. By irreducibility of $W, \Pi \subseteq U$ and (d) follows.

1.14. Let $\Delta \subseteq \Phi$ with $\Delta=\Pi_{W^{\prime}}$ where $W^{\prime}:=W_{\Delta}$ (for instance, $\Delta \subseteq \Pi$ ). Then $\Delta$ is said to have a certain property of based root systems (e.g. be irreducible, have $n$ components, be irreducible of affine type etc) if the based root system $\left(\Phi_{W^{\prime}}, \Delta\right)$ has that property. If $W^{\prime}$ is finite, its longest element is denoted $w_{\Delta}$ or by $w_{I}$ where $I:=\left\{s_{\alpha} \mid \alpha \in \Delta\right\}$ is the set of its simple reflections.

Sometimes a reflection subgroup $W^{\prime}$ (resp., subset $I$ of $S$ ) will be said to have a certain property of root systems (be irreducible etc) if $\Pi_{W^{\prime}}\left(\right.$ resp., $\Pi_{I}$ ) has that property; we may abuse terminology by doing this even when that property is a property of root systems and not just of the corresponding Coxeter group $W^{\prime}$ (resp., $\left.W_{I}\right)$. For example, see Remark 4.5(i). We view the vertex set of the Coxeter graph of a reflection subgroup $W^{\prime}$ as either $\Pi_{W^{\prime}}$ or $\chi\left(W^{\prime}\right)$ as convenience dictates, and often identify full subgraphs of the Coxeter graph with their vertex set. Thus, we refer to the connected (or equivalently, irreducible or indecomposable) components of $\Pi_{W^{\prime}}$ or $\chi\left(W^{\prime}\right)$ or $W^{\prime}$ (they are, respectively, subsets of $\Pi_{W^{\prime}}$, subsets of $\chi\left(W^{\prime}\right)$, and subgroups of $\left.W^{\prime}\right)$.

Subsets $\Delta, \Delta^{\prime}$ of $\Phi$ are defined to be separated if $\Delta \perp \Delta^{\prime}$. Similarly, subsets $I, J$ of $S$ are separated if $\Pi_{I}$ and $\Pi_{J}$ are separated. A subset $I$ of $S$ (resp., $\Delta$ of $\Pi$ ) is said to be of finite type if $W_{I}$ (resp., $W_{\Delta}$ ) is finite. 
1.15. The basic fact below, due to Deodhar [12] (see also [7]), will be required several times in this paper.

Theorem. Suppose that $\Delta, \Gamma \subseteq \Pi$ and $w \in W$ with $w(\Delta)=\Gamma$. Then there exist $n \in \mathbb{N}, w_{1}, \ldots, w_{n} \in W, \Delta_{0}, \ldots, \Delta_{n} \subseteq \Pi$ and $\alpha_{1}, \ldots, \alpha_{n} \in \Pi$, such that

(i) $\Delta_{0}=\Delta$ and $\Delta_{n}=\Gamma$.

(ii) For $i=1, \ldots, n, \alpha_{i} \notin \Delta_{i-1}$, the connected component $\Gamma_{i}$ of $\Delta_{i-1} \cup\left\{\alpha_{i}\right\}$ containing $\alpha_{i}$ is of finite type, and $w_{i}=w_{\Gamma_{i}} w_{\Gamma_{i} \backslash\left\{\alpha_{i}\right\}}$.

(iii) $\Delta_{i}=w_{i} \Delta_{i-1}$ for $i=1, \ldots, n$.

(iv) $w=w_{n} \cdots w_{1}$ and $l(w)=l\left(w_{1}\right)+\cdots+l\left(w_{n}\right)$.

Remarks. Suppose above that $\Delta$ has the property that if $\alpha \in \Pi \backslash\left(\Delta \cup\left(\Pi \cap \Delta^{\perp}\right)\right)$, then the connected component of $\Delta \cup\{\alpha\}$ containing $\alpha$ is infinite. Then above, each $\alpha_{i} \in \Pi \cap \Delta^{\perp}$ and $w_{i}=s_{\alpha_{i}}$, so $w \in W_{\Pi \cap \Delta^{\perp}}$ and $\Gamma=w(\Delta)=\Delta$ (in fact, $w$ fixes $\Delta$ pointwise). This applies in particular if every component of $\Delta$ is infinite (cf. 7] and [16, 1.23]).

1.16. The following is well known, but we provide a proof for ease of reference.

Lemma. Let $\alpha_{0}, \alpha_{1}, \ldots, \alpha_{n} \in \Pi$ be distinct. Assume that for each $i>0$, there is some $j<i$ with $\left\langle\alpha_{i}, \alpha_{j}\right\rangle \neq 0$. Let $\alpha:=s_{\alpha_{n}} \cdots s_{\alpha_{1}}\left(\alpha_{0}\right)$. Then

(a) $\alpha=\sum_{i=0}^{n} c_{i} \alpha_{i}$ for some $c_{i} \in \mathbb{R}_{>0}$.

(b) $l\left(s_{\alpha}\right)=2 n+1$.

(c) $s_{\alpha}=s_{\alpha_{n}} \cdots s_{\alpha_{1}} s_{\alpha_{0}} s_{\alpha_{1}} \cdots s_{\alpha_{n}}$ is a reduced expression for $s_{\alpha}$.

Proof. This is proved by induction on $n$ as follows. For $n=0$, the assertions are trivial. Suppose inductively that $n>0$. Write $\beta:=s_{\alpha_{n-1}} \cdots s_{\alpha_{1}}\left(\alpha_{0}\right)=\sum_{i=0}^{n-1} c_{i} \alpha_{i}$ for some $c_{i} \in \mathbb{R}_{>0}$. Then $c_{n}:=-\left\langle\beta, \alpha_{n}^{\vee}\right\rangle=-\sum_{i=0}^{n-1} c_{i}\left\langle\alpha_{i}, \alpha_{n}^{\vee}\right\rangle>0$ since $c_{0}, \ldots, c_{n-1}>0,\left\langle\alpha_{i}, \alpha_{n}^{\vee}\right\rangle \leq 0$ for all $i<n$ since $\alpha_{i} \neq \alpha_{n}$ and $\left\langle\alpha_{i}, \alpha_{n}^{\vee}\right\rangle<0$ for some $i<n$ by assumption. It follows that $\alpha=s_{\alpha_{n}}(\beta)=\beta+c_{n} \alpha_{n}$ is as in (a). Noting that $\beta \neq \alpha_{n}$, 3. Lemma 3.4] implies that $l\left(s_{\alpha}\right)=l\left(s_{\beta}\right)+2$. Since $s_{\alpha}=s_{\alpha_{n}} s_{\beta} s_{\alpha_{n}}$, (b) and (c) follow by induction.

1.17. The next lemma is for use in the proof of Lemma 2.10.

Lemma. Let $I, J \subseteq S$. Then the following conditions are equivalent:

(i) $W=W_{I} W_{J}$.

(ii) $T=\left(T \cap W_{I}\right) \cup\left(T \cap W_{J}\right)$.

(iii) $\Phi=\Phi_{I} \cup \Phi_{J}$.

(iv) For every irreducible component $\Delta$ of $\Pi$, either $\Delta$ is contained in $\Pi_{I}$ or $\Delta$ is contained in $\Pi_{J}$.

Proof. We first show that (i) implies (ii). Assume that (i) holds. Let $t \in T$. We may write $t=u v$ where $u \in W_{I}$ and $v \in W_{J}$. Choosing reduced expressions for $u$ and $v$ and substituting in $t=u v$ gives an expression for $t$ which may be cancelled (by repeated deletion of appropriate pairs of simple reflections) to a reduced expression $t=s_{1} \ldots s_{m}$ where $s_{1}, \ldots, s_{n} \in I$ and $s_{n+1}, \ldots, s_{m} \in J$. Note that $m=l(t)=$ $2 k+1$ for some $k$. If $n \geq k+1$, we have $t=s_{1} \cdots s_{k} s_{k+1} s_{k} \cdots s_{1} \in W_{I}$ by [17, (2.7)]. On the other hand, if $n \leq k$, then similarly $t=t^{-1}=s_{2 k+1} \cdots s_{1}=$ $s_{2 k+1} \cdots s_{k+2} s_{k+1} s_{k+2} \cdots s_{2 k+1} \in W_{J}$. Hence (ii) holds.

The equivalence of (ii) and (iii) is clear. Next we show that (iii) implies (iv). Assume that (iii) holds but (iv) doesn't. Suppose that $\Delta$ is an irreducible component of $\Pi$ which is contained in neither $\Pi_{I}$ nor $\Pi_{J}$. Then there is some finite subset 
$\Delta^{\prime}$ of $\Delta$ which is connected but contained in neither $\Pi_{I}$ nor $\Pi_{J}$. By Lemma 1.16 , there is a root $\alpha \in \Phi$ such that $s_{\alpha}$ has a reduced expression containing $s_{\beta}$ for all $\beta \in \Delta^{\prime}$. Then $\alpha \notin \Phi_{I} \cup \Phi_{J}$, a contradiction. This completes the proof that (iii) implies (iv).

Finally, we show that (iv) implies (i). Let $w \in W$. Write $w=w_{1} \ldots w_{n}$ where $w_{i} \in W_{\Delta_{i}}$ for some irreducible component $\Delta_{i}$ of $\Pi$. Since $w_{i}$ and $w_{j}$ commute if $\Delta_{i} \neq \Delta_{j}$, it may be assumed that the $\Delta_{i}$ are pairwise distinct and, by reindexing if necessary, it may also be assumed that $\Delta_{1}, \ldots, \Delta_{m}$ are contained in $\Pi_{I}$ while $\Delta_{m+1}, \ldots, \Delta_{n}$ are contained in $\Pi_{J}$. Then $w_{1}, \ldots, w_{m} \in W_{I}$ and $w_{m+1}, \ldots, w_{n} \in$ $W_{J}$ so $w \in W_{I} W_{J}$ as required to prove that (iv) implies (i).

1.18. The proposition below collects assorted useful facts which show how certain subsets of roots (orbits, complements of parabolic subsystems and cofinite subsets of the roots) may be regarded as being "large" in various natural senses.

Proposition. Suppose that $(W, S)$ is irreducible.

(a) Let $\alpha \in \Phi$ and let $Z:=\{w \in W \mid w(\alpha)=\alpha\}$ denote the stabilizer of $\alpha$ in $W$. Then $\mathbb{R}(W \alpha)=\mathbb{R} \Phi, W$ acts faithfully on the $W$-orbit $W \alpha$ and $\cap_{w \in W} w Z w^{-1}=\left\{1_{W}\right\}$. In particular, if $W \alpha$ is finite, then $W$ is finite.

(b) Let $I \subsetneq S$ and $\Gamma:=\Phi \backslash \Phi_{I}$ Then $W_{\Gamma}=W, W \Gamma=\Phi$, and $\mathbb{R} \Gamma=\mathbb{R} \Phi$.

(c) Suppose that $W$ is infinite. If $\Delta \subseteq \Phi_{+}$with $\left|\Phi_{+} \backslash \Delta\right|$ finite, then $W_{\Delta}=W$, $W \Delta=\Phi$ and $\mathbb{R} \Delta=\mathbb{R} \Pi$.

Proof. To prove (a), assume without loss of generality that $\alpha \in \Pi$. Since $\alpha \not \perp \Pi$ by irreducibility of $W$, one has aff $(W \alpha)=\alpha+\mathbb{R} \Pi=\mathbb{R} \Pi$ by Lemma $1.13(\mathrm{~d})$. Hence $\mathbb{R}(W \alpha)=\mathbb{R} \Pi=\mathbb{R} \Phi$, and the rest of (a) follows since $W$ acts faithfully on $\Phi$.

To prove (b), it will suffice to show that $\Pi \subseteq W_{\Gamma} \Gamma$. Fix $\alpha \in \Pi \backslash \Pi_{I}$ and let $\beta \in \Pi$. By irreducibility of $W$, there exist $n \in \mathbb{N}_{>0}$, and a sequence $\alpha=\alpha_{0}, \ldots, \alpha_{n}=\beta$ of distinct simple roots such that $\left\langle\alpha_{i-1}, \alpha_{i}\right\rangle \neq 0$ for $i=1, \ldots, n$. Define $\gamma_{i}:=$ $s_{\alpha_{0}} \cdots s_{\alpha_{i-1}}\left(\alpha_{i}\right)$ for $i=0, \ldots, n$. By Lemma 1.16, $s_{\gamma_{i}}$ has a reduced expression containing $s_{\alpha_{0}}$ and hence $\gamma_{i} \in \Phi \backslash \Phi_{I}=\Gamma$. It follows by induction on $i$ that $\alpha_{i} \in W_{\Gamma} \Gamma$ and $s_{\alpha_{i}} \in W_{\Gamma}$ for $i=0, \ldots, n$. In particular, $\beta=\alpha_{n} \in W_{\Gamma} \Gamma$. Since $\beta \in \Pi$ is arbitrary, this gives $\Pi \subseteq W_{\Gamma} \Gamma$ as required to complete the proof of the first statement in (b).

If there is a proper standard parabolic subgroup $W_{I}$ with $\Phi_{+} \backslash \Delta \subseteq \Phi_{I}$, then (c) follows from (b) since $\Delta \supseteq \Phi_{+} \backslash \Phi_{I}$. Such a subgroup $W_{I}$ certainly exists if $W$ is of infinite rank, so we may and do assume for the rest of this proof that $\Pi$ is finite. It is easy to check that (c) holds if $(W, S)$ is infinite dihedral, and we now reduce to that case. In fact, let $\beta \in \Phi$. Since $(W, S)$ is irreducible of finite rank and infinite, it follows from [31, Proposition 3.13] that there is some $\alpha \in \Phi$ such that $W^{\prime}:=\left\langle s_{\alpha}, s_{\beta}\right\rangle$ is infinite. Let $\Psi:=\Phi_{W^{\prime}}, \Gamma:=\Delta \cap \Psi \subseteq \Psi_{+}$. Note that $\Psi_{+} \backslash \Gamma \subseteq \Phi_{+} \backslash \Delta$ is a finite set. From the dihedral case, it follows that $s_{\beta} \in W_{\Gamma} \subseteq W_{\Delta}, \beta \in W^{\prime} \Gamma \subseteq W \Delta$ and $\beta \in \mathbb{R} \Gamma \subseteq \mathbb{R} \Delta$. Since $\beta \in \Phi$ is arbitrary, the conclusions of (c) now follow trivially.

1.19. Despite its formulation below, the next fact is purely graph-theoretic in nature. Though a more general fact is established in [50], we provide for the reader's convenience a self-contained proof of the special case used here.

Lemma. Suppose $W$ is irreducible, $S$ is infinite and the Coxeter graph is locally finite (i.e. for each $\alpha \in \Pi$ there are only finitely many $\beta$ in $\Pi$ such that $\langle\alpha, \beta\rangle \neq 0$ ). 
Fix $\alpha \in \Pi$. Then there is an infinite sequence $\alpha=\alpha_{0}, \alpha_{1}, \ldots$ of distinct roots in $\Pi$ such that for $i<j \in \mathbb{N}$, the distance from $\alpha_{i}$ to $\alpha_{j}$ in the Coxeter graph is $j-i$. In particular, if $i<j$, then $\left\langle\alpha_{i}, \alpha_{j}\right\rangle \neq 0$ if and only if $j=i+1$.

Proof. For $\gamma, \beta \in \Pi$, a path from $\gamma$ to $\beta$ is a sequence $\gamma=\gamma_{0}, \ldots, \gamma_{n}=\beta$ in $\Pi$ with $\left\langle\gamma_{i-1}, \gamma_{i}\right\rangle \neq 0$ for $i=1, \ldots, n$. Such paths exist by irreducibility of $W$. Define $d(\gamma, \beta)$ to be the minimum length $n$ of all such paths from $\gamma$ to $\beta$. Note that if the above path satisfies $n=d(\gamma, \beta)$, then $d\left(\gamma_{i}, \gamma_{j}\right)=j-i$ for all $0 \leq i \leq j \leq n$.

For each $n \in \mathbb{N}$, let $\Pi_{n}:=\{\beta \in \Pi \mid d(\alpha, \beta)=n\}$. If $\beta \in \Pi_{n}$, we may choose a path $\alpha=\alpha_{0}, \ldots, \alpha_{n}=\beta$ from $\alpha$ to $\beta$, and one necessarily has $\alpha_{i} \in \Pi_{i}$ (and so $\Pi_{i} \neq \emptyset$ ) for $i=0, \ldots, n$. Using local finiteness of the Coxeter graph, it follows by induction on $n$ that $\Pi_{n}$ is finite for all $n$, since for $n>0$, any root in $\Pi_{n}$ is the endpoint of one of the (finitely many, by induction) edges with one vertex in $\Pi_{n-1}$. Since $\Pi$ is infinite, there is for any $m \in \mathbb{N}$ some $n>m$ with $\Pi_{n} \neq \emptyset$, and as above, this implies $\Pi_{m} \neq \emptyset$. Hence each $\Pi_{n}$ is a non-empty finite set. Note $\Pi_{0}=\{\alpha\}$. Give $\Pi_{n}$ the discrete topology and endow the product set $X=\prod_{n \in \mathbb{N}} \Pi_{n}$ with the product topology. Thus, $X$ is a compact Hausdorff space. For each $n \in \mathbb{N}$, let

$$
X_{n}:=\left\{\left(\alpha_{0}, \alpha_{1}, \ldots\right) \in X \mid \alpha_{0}, \alpha_{1}, \ldots, \alpha_{n} \text { is a path from } \alpha=\alpha_{0} \text { to } \alpha_{n}\right\} .
$$

One has for $n \in \mathbb{N}$ that $X_{n} \neq \emptyset$; for one may choose in turn $\alpha_{n} \in \Pi_{n}$, a path $\alpha_{0}, \ldots, \alpha_{n}$ from $\alpha$ to $\alpha_{n}$ and then arbitrary $\alpha_{m} \in \Pi_{m}$ for $m>n$ to obtain an element $\left(\alpha_{0}, \alpha_{1}, \ldots\right) \in X_{n}$. Also, one easily sees from the definition of product topology that $X \backslash X_{n}$ is open in $X$ and hence that $X_{n}$ is closed in $X$. Obviously, $X_{0} \supseteq X_{1} \supseteq \ldots$ By compactness of $X, Y:=\cap_{n \in \mathbb{N}} X_{n} \neq \emptyset$. Let $\left(\alpha_{0}, \alpha_{1}, \ldots\right) \in Y$. From above, $d\left(\alpha_{i}, \alpha_{j}\right)=j-i$ for all $i<j$ in $\mathbb{N}$. In particular, if $\left\langle\alpha_{i}, \alpha_{j}\right\rangle \neq 0$, then $1=d\left(\alpha_{i}, \alpha_{j}\right)=j-i$, and so the sequence $\alpha_{0}, \alpha_{1}, \ldots$ has the required properties.

1.20. In the special case that $S$ is finite, the next result has been proved by Deodhar (and (a) independently by Howlett; see [12, Proposition 4.2(x) and Remark 4.3]).

Lemma. Suppose that $(W, S)$ is an irreducible Coxeter system and $I \subsetneq S$

(a) If $W / W_{I}$ is finite, then $W$ is finite.

(b) If $\Phi \backslash \Phi_{I}$ is finite, then $W$ is finite.

Proof. First we prove (a). Using the references preceding the statement of the lemma, suppose without loss of generality that $S$ is infinite. We shall obtain a contradiction after first showing that $(W, S)$ is of locally finite type i.e. that $W_{K}$ is finite for every finite subset $K$ of $S$. (This notion should not be confused with that of $(W, S)$ having locally finite Coxeter graph in 1.19 . We remark that it follows easily from the classification of finite Coxeter groups that the possible types of infinite rank, irreducible, Coxeter systems of locally finite type are $A_{\infty}, A_{\infty, \infty}, B_{\infty}$ or $D_{\infty}$; see [33] or [16] for the notation, or [42, Figure 1] where $A_{\infty}, A_{\infty, \infty}$ are denoted as $A_{\infty}^{(1)}, A_{\infty}^{(2)}$. This classification can be used instead of the reference to Lemma 1.19 in the proof below.)

Since $W^{I}$ is a set of coset representatives for $W / W_{I}$, it is finite. Hence there is some standard parabolic subgroup $W_{J}$, with $J \subseteq S$ finite, such that $W^{I} \subseteq W_{J}$. By irreducibility of $W$, one may assume without loss of generality that $W_{J}$ is irreducible and $J$ is sufficiently large that $K \subseteq J$ and $J \cap I \subsetneq J$. Then $W_{J} / W_{J \cap I} \cong W_{J}^{J \cap I} \subseteq$ $W^{I}$ is finite, which implies $W_{J}$ is finite by the special case. Since $K \subseteq J, W_{K}$ is 
finite too. Since the vertex degrees in Coxeter graphs of finite Coxeter systems are bounded above, the Coxeter graph of $(W, S)$ is locally finite. Lemma 1.19 implies that there is for any $\alpha \in \Pi$ a sequence $\alpha=\alpha_{0}, \alpha_{1}, \ldots, \alpha_{n}, \ldots$ of distinct simple roots such that $\left\langle\alpha_{n-1}, \alpha_{n}\right\rangle \neq 0$ for all $n \geq 1$. Choose $\alpha \in \Pi \backslash \Pi_{I}$. For all $n \in \mathbb{N}$, the element $w_{n}:=s_{\alpha_{n}} \cdots s_{\alpha_{0}}$ of $W$ is of length $n+1$ (using Lemma 1.16, for instance). Clearly, $w_{n}$ has a unique reduced expression. Since $s_{\alpha_{0}} \notin I$, the elements $w_{n}$, for $n \in \mathbb{N}$, are distinct elements of $W^{I}$. Hence $W^{I}$ is infinite, which is a contradiction.

Now (b) follows from (a) in the same way as its special case in which $S$ is finite, by the argument of [12, Remark 4.4]. Alternatively, note that $\Phi \backslash \Phi_{I}$ finite implies $W=W_{\Phi \backslash \Phi_{I}}$ is finitely generated by Proposition 1.18 , so $S$ is finite (since it is a minimal set of generators of $W$ ) and $W$ is finite by the result of [12, Remark 4.4].

1.21. The next result extends [14, Lemma 1.22].

Lemma. Assume that $\Pi$ is finite. Let $\rho: V \rightarrow \mathbb{R}$ be a linear function such that $\rho(\Pi) \subseteq \mathbb{R}_{>0}$.

(a) There is a positive scalar $\epsilon_{0}$ such that for any two non-orthogonal roots $\alpha, \beta \in \Phi$ one has $\left|\left\langle\alpha, \beta^{\vee}\right\rangle\right| \geq \epsilon_{0}$.

(b) Let $\epsilon:=\min \left(\left\{\rho(\alpha) \epsilon_{0} / 2 \mid \alpha \in \Pi\right\}\right.$. Then for all $\beta \in \Phi_{+}, \rho(\beta) \geq \epsilon l\left(s_{\beta}\right)$.

Proof. For (a), see [14, Lemma 1.22(i)]. Part (b) is proved by induction on $l\left(s_{\beta}\right)$ in the same way as [14, Lemma 1.22(ii)], making use of [3, Proposition 3.4].

1.22. Roots in the fundamental chamber may be regarded as general analogues of highest (long or short) roots in root systems of irreducible finite Weyl groups. For irreducible $W$, their existence is equivalent to finiteness of $W$, as the next result shows. There are generalizations concerning orbits of standard parabolic subgroups of $W$ on $\Phi$ which we do not go into here.

Corollary. (a) If $W$ is finite, then the $W$-orbit of any root contains a unique element of $\Phi \cap \mathscr{C}_{W}$.

(b) Suppose that $\alpha \in \Phi \cap \mathscr{C}_{W}$. Then $\alpha \in \Phi_{+}$. If, further, $(W, S)$ is irreducible, then $W$ is finite.

Proof. Part (a) follows from 1.10(h),(c). Now let $\alpha \in \Phi \cap \mathscr{C}$. Write $\alpha=\sum_{\gamma \in \Gamma} c_{\gamma} \gamma$ where $\Gamma \subseteq \Pi$, $\Gamma$ is finite and either $c_{\gamma}<0$ for all $\gamma \in \Gamma$ or $c_{\gamma}>0$ for all $\gamma \in \Gamma$. Since $1=\langle\alpha, \alpha\rangle=\sum_{\gamma \in \Gamma} c_{\gamma}\langle\gamma, \alpha\rangle$ where each $\langle\gamma, \alpha\rangle \geq 0$ (because $\alpha \in \mathscr{C}$ ), it follows that we must have $c_{\gamma}>0$ for all $\gamma \in \Gamma$, and so $\alpha \in \Phi_{+}$.

Now assume that $(W, S)$ is irreducible. We claim that $\Gamma=\Pi$. If not, by irreducibility of $\Pi$, there is some $\beta \in \Pi \backslash \Gamma$ and $\gamma_{0} \in \Gamma$ such that $\left\langle\gamma_{0}, \beta\right\rangle<0$. One also has $\langle\gamma, \beta\rangle \leq 0$ for all $\gamma \in \Gamma$. Since $\alpha \in \mathscr{C}$, it follows that

$$
0 \leq\langle\beta, \alpha\rangle=\sum_{\gamma \in \Gamma} c_{\gamma}\langle\beta, \gamma\rangle \leq c_{\gamma_{0}}\left\langle\beta, \gamma_{0}\right\rangle<0
$$

a contradiction.

Since $\Pi=\Gamma$ is finite, we may choose $\rho, \epsilon$ as in Lemma 1.21. By Lemma 1.6, for any $w \in W$, one has $\alpha-w(\alpha) \in \mathbb{R}_{\geq 0} \Pi$ and so $\rho(w \alpha) \leq \rho(\alpha)$. Assume that $w \alpha \in \Phi_{+}$. By Lemma $1.21 l\left(s_{w(\alpha)}\right) \leq \epsilon^{-1} \rho(w \alpha) \leq \epsilon^{-1} \rho(\alpha)=: N$. But there are at most $|\Pi|^{m}<\infty$ elements of $W$ of length $m$ for any $m \in \mathbb{N}_{\leq N}$. This implies that $W \alpha \cap \Phi_{+}$is finite, and hence $W \alpha=\left(W \alpha \cap \Phi_{+}\right) \cup-\left(W \alpha \cap \Phi_{+}\right)$is finite. By Proposition 1.18(a), it follows that $W$ is finite. 


\section{FACIAL SUBGROUPS}

This section contains basic facts about facial subgroups of $W$, which are defined as the stabilizers of points of the Tits cone. Some of these facts are proved under stronger assumptions in [4], 49], and [34].

2.1. A subset $\Pi^{\prime}$ of $\Pi$ is said to be a facial subset of $\Pi$, or said to be facial, if $\Pi^{\prime}=\Pi \cap v^{\perp}$ for some $v \in \mathscr{C}$. Obviously, $\Pi=\Pi \cap 0^{\perp}$ itself is facial. It is not clear without additional assumptions on the root system if there are other facial subsets.

If $\Pi^{\prime}$ is facial, then $I=\left\langle s_{\alpha} \mid \alpha \in \Pi^{\prime}\right\rangle$ is said to be a facial subset of $S$ and $W_{I}$ is called a standard facial subgroup. Using that $\mathscr{C}$ is a cone, one sees that a finite intersection of facial subsets of $\Pi$ (resp., facial subsets of $S$, resp., standard facial subgroups) is again a facial subset of $\Pi$ (resp., facial subset of $S$, resp., standard facial subgroup). A facial subgroup of $W$ is defined to be to be a conjugate in $W$ of a standard facial subgroup.

Remarks. The facial subsets correspond naturally to the exposed faces (with respect to the semidual pair of cones $\left.\left(\mathbb{R}_{\geq 0} \Pi, \mathscr{C}\right)\right)$ of $\mathbb{R} \Pi$ in the sense of Appendix A. Certain subtleties in the notion of facial subsets reflect subtleties in the notion of exposed faces. For instance, compare Remark 2.7 and Lemma 4.2 (f) with the facts that, for general semidual pairs of cones, exposed faces of exposed faces need not be exposed faces, whereas this does hold for dual pairs of polyhedral cones. The notion of facial subset, subgroup etc may change under restriction or extension of quadratic space.

2.2. For $p \in V$, let $W_{p}$ denote the stabilizer of $v$ in $W$. Then for all $w \in W$, one has $W_{w p}=w W_{p} w^{-1}$.

Lemma. Let $q \in \mathscr{X}$. Let $w \in W$ be such that $p:=w^{-1} q \in \mathscr{C}$. Let $J$ be the facial subset of $S$ with $\Pi_{J}=\Pi \cap p^{\perp}$.

(a) $W_{p}=W_{J}, \chi\left(W_{p}\right)=J, \Phi_{W_{p}}=\Phi \cap p^{\perp}$ and $\Pi_{W_{p}}=\Pi \cap p^{\perp}$.

(b) $W_{q}=w W_{J} w^{-1}$ with $\Phi_{W_{q}}=\Phi \cap q^{\perp}$.

Proof. By $1.10(\mathrm{c}), W_{p}=W_{J}$. The definition of $\chi\left(W_{J}\right)$ easily implies that $J \subseteq$ $\chi\left(W_{J}\right)$. Hence $\chi\left(W_{J}\right)=J$, since (see [4, Ch IV]) any set of Coxeter generators of $W_{J}$, such as $\chi\left(W_{J}\right)$, is a minimal set of generators of $W_{J}$. From $\chi\left(W_{J}\right)=J$, it follows by definition that $\Pi_{W_{p}}=\Pi_{J}=\Pi \cap p^{\perp}$. The assertion in (a) that $\Phi_{W_{p}}=\Phi \cap p^{\perp}$, and (b), follow from $1.10(\mathrm{~d})$.

2.3. For a subset $I$ of $S$, let $\mathscr{C}(I):=\left\{v \in \mathscr{C} \mid v^{\perp} \cap \Pi=\Pi_{I}\right\}$, which is a cone in $V$. A non-empty set of the form $w \mathscr{C}(I)$ with $w \in W$ and $I \subseteq S$ is called a facet of $\mathscr{X}$. (Note that this conflicts in some situations with a common usage of the word facet for a codimension one face of a cone; in general, $w \mathscr{C}(I)$ is not a face of $\mathscr{X}$.)

For any $\phi \in V$, and a relation $\prec$ on $\mathbb{R}$ which is one of the standard equality, order or inequality relations $=, \leq,<, \geq$, >, or $\neq$ let $H_{\phi}^{\prec}:=\{v \in V \mid\langle v, \phi\rangle \prec 0\}$.

Basic properties of facial subgroups and facets are given in the following Lemma.

Lemma. (a) For $I \subseteq S, \mathscr{C}(I) \neq \emptyset$ if and only if $I$ is facial.

(b) Suppose that $I, J$ are facial subsets of $S$ and $x, y \in W$. Then the intersection $x \mathscr{C}(I) \cap y \mathscr{C}(J)$ is non-empty if and only if $I=J$ and $x^{-1} y \in W_{J}$, in which case $y x^{-1} \in x W_{J} x^{-1}=y W_{J} y^{-1}$ fixes $x \mathscr{C}(I)=y \mathscr{C}(J)$ pointwise.

(c) The stabilizers of the points of $\mathscr{C}$ (resp., $\mathscr{X}$ ) are exactly the standard facial subgroups (resp., facial subgroups). 
(d) Any (standard) facial subgroup of $W$ is (standard) parabolic.

(e) The cone $\mathscr{X}$ is the disjoint union of its facets.

(f) The facets of $\mathscr{X}$ are the non-empty intersections of the form $\cap_{\alpha \in \Phi_{+}} H_{\alpha}^{\prec \alpha}$ such that for $\alpha \in \Phi_{+}, \prec_{\alpha}$ is one of the relations $=,\langle$ or $\rangle$, with only finitely many $\alpha$ for which $\prec_{\alpha}$ is equal to $<$.

(g) The conical hull of any finite union of facets of $\mathscr{X}$ meets only finitely many facets. In particular, the conical hull of any finite subset of $\mathscr{X}$ meets only finitely many facets.

Proof. Part (a) follows from the definition of facial subsets of $\Pi$, (b) follows from Lemma 1.10. (c)-(d) follow from Lemma 2.2 and (e) follows from (b) (cf. 34]).

Now we prove (f). If $v \in F:=\cap_{\alpha \in \Phi_{+}} H_{\alpha}^{\prec}$, then for all $\alpha \in \Phi_{+}$, one has $\langle v, \alpha\rangle \prec_{\alpha} 0$ and hence the relations $\prec_{\alpha}$ (amongst $=,<,>$ ) are uniquely determined. This implies that the intersections as in (f) are pairwise disjoint, and they are contained in $\mathscr{X}$ by Lemma 1.10 Let $w \in W$. Choose relations $\prec_{\alpha}^{\prime}$ (amongst $=,<$, $>$ ) satisfying $\langle w v, \alpha\rangle \prec_{\alpha}^{\prime} 0$. Note that $\prec_{\alpha}^{\prime}$ and $\prec_{w^{-1} \alpha}$ coincide if $w^{-1}(\alpha) \in \Phi_{+}$. If $w^{-1}(\alpha) \in \Phi_{-}$, then $\prec_{\alpha}^{\prime}$ and $\prec_{-w^{-1} \alpha}$ are either both $=$, or one of them is $<$ and the other is $>$. It follows that $w\left(\cap_{\alpha \in \Phi_{+}} H_{\alpha}^{\prec \alpha}\right)=\cap_{\alpha \in \Phi_{+}} H_{\alpha}^{\prec^{\prime}}$. By Lemma 1.10 only finitely many of the $\prec_{\alpha}^{\prime}$ are equal to $<$. This implies that the set of non-empty intersections as in (f) is $W$-stable. Hence it will suffice to show that the facets of $\mathscr{X}$ which contain a point of $\mathscr{C}$ are precisely the non-empty intersections $F$ with all $\prec_{\alpha}$ amongst $=,>$. But if $v \in \mathscr{C}$, then the unique facet containing $v$ is $\mathscr{C}(I)$ where $\Pi_{I}=\{\alpha \in \Pi \mid\langle v, \alpha\rangle=0\}$ and the unique intersection as in (f) which contains $v$ is $F:=\cap_{\alpha \in \Phi_{+}} H_{\alpha}^{\prec \alpha}$ where $\langle v, \alpha\rangle \prec_{\alpha} 0$ with $\prec_{\alpha}$ either $=$ or $>$. Consider a root $\alpha=\sum_{\beta \in \Pi} c_{\beta} \beta \in \Phi$ with all $c_{\beta} \geq 0$. Then $\prec_{\alpha}$ is $=$ if and only if $c_{\beta}=0$ for all $\beta \in \Pi \backslash \Pi_{I}$. It is clear then that

$$
F=\left\{v \in \mathscr{C} \mid\langle v, \alpha\rangle=0 \text { for all } \alpha \in \Pi_{I}\right\}=\mathscr{C}(I)
$$

completing the proof of (f).

For the first claim of $(\mathrm{g})$, let $F_{1}, \ldots, F_{n}$ be facets. Write $F_{i}=\cap_{\alpha \in \Phi_{+}} H_{\alpha}^{\prec \alpha, i}$ as in (f). It will suffice to show that there are only finitely many facets $F=\cap_{\alpha \in \Phi_{+}} H_{\alpha}^{\prec \alpha}$ containing some point $x=\sum_{i=1}^{n} \lambda_{i} x_{i}$ with $x_{i} \in F_{i}$ and all $\lambda_{i}>0$. Assume $x \in F$, and let $\alpha \in \Phi_{+}$. Clearly, $\prec_{\alpha}$ is $=$ if all $\prec_{\alpha, i}$ are $=$, while $\prec_{\alpha}$ is $>$ if no $\prec_{\alpha, i}$ is $<$ and at least one $\prec_{\alpha, i}$ is $>$. There are only finitely many $\alpha \in \Phi_{+}$which do not fall under one of those two cases, namely the $\alpha$ for which some $\prec_{\alpha, i}$ is $<$, and for each of these, there are at most three possibilities $(=,<,>)$ for $\prec_{\alpha}$. It follows that there are only finitely many possible families $\left(\prec_{\alpha}\right)_{\alpha \in \Phi_{+}}$and so only finitely many possible $F$. This proves the first assertion of (g), and the second follows since each point of $\mathscr{X}$ lies in some facet (in the case of two points, compare 4 , Ch V, $\S 4$, Prop $6])$.

Remarks. If $\Pi$ is linearly independent and $(\Phi, \Pi)$ on $(V,\langle-,-\rangle)$ is ample (see Remark 1.3(2)), then the classes of (standard) facial subgroups and (standard) parabolic subgroups coincide.

2.4. Parts (a)-(c) at least of the following are trivial or well-known in case $\Pi$ is linearly independent (see [7, Proposition 3.3]).

Lemma. Let $I$ be a facial subset of $S$. Fix $a \in \mathscr{C}$ such that $\Pi_{I}=\Pi \cap a^{\perp}$. Then

(a) $\mathbb{R}_{\geq 0} \Pi \cap a^{\perp}=\mathbb{R}_{\geq 0} \Pi \cap \mathbb{R}_{I}=\mathbb{R}_{\geq 0} \Pi_{I}$. 
(b) $\Phi \cap a^{\perp}=\Phi \cap \mathbb{R} \Pi_{I}=W_{I} \Pi_{I}=\Phi_{W_{I}}$.

(c) $\Phi_{+} \cap a^{\perp}=\Phi_{+} \cap \mathbb{R}_{I}=\Phi_{+} \cap \mathbb{R}_{\geq 0} \Pi_{I}=W_{I} \Pi_{I} \cap \Phi_{+}=\Phi_{W_{I},+}$.

(d) If $k \in \mathscr{C}, w \in W$ and $k-w k \in \mathbb{R} \Pi_{I}$, then $w k=w^{\prime} k$ for some $w^{\prime} \in W_{I}$.

Proof. The statement obtained by replacing "=" by " $\supseteq$ " in (a) is clear. For the reverse inclusions, suppose that $v=\sum_{\alpha \in \Pi} c_{\alpha} \alpha \in \mathbb{R}_{\geq 0} \Pi \cap a^{\perp}$, where all $c_{\alpha} \geq 0$. We have $0=\langle v, a\rangle=\sum_{\alpha \in \Pi} c_{\alpha}\langle\alpha, a\rangle$. Since $\langle\alpha, a\rangle \geq 0$ for all $\alpha \in \Pi$, we get $c_{\alpha}=0$ if $\langle\alpha, a\rangle \neq 0$ i.e. if $\alpha \notin \Pi_{I}$, which proves (a).

In (c), the first two equalities follow by intersecting each part of (a) with $\Phi_{+}$. It is also clear that $\Phi_{W_{I},+}=W_{I} \Pi_{I} \cap \Phi_{+} \subseteq \Phi_{+} \cap \mathbb{R}_{>0} \Pi_{I}$. For the reverse inclusion, let $\beta \in \Phi_{+} \cap \mathbb{R}_{\geq 0} \Pi_{I}$. Write $\beta=\sum_{\alpha \in \Pi_{I}} c_{\alpha} \alpha$ with all $c_{\alpha} \geq 0$. If $\beta \in \Pi$, then $\beta \in \Pi_{I}$, else $\left\langle\beta, \Pi_{I}\right\rangle \subseteq \mathbb{R}_{\leq 0}$ and

$$
1=\langle\beta, \beta\rangle=\sum_{\alpha \in \Pi_{I}} c_{\alpha}\langle\beta, \alpha\rangle \leq 0 .
$$

We show that $\beta \in W_{I} \Pi_{I}$ in general by induction on $l\left(s_{\beta}\right)$. Assume $l\left(s_{\beta}\right)>1$. Using [3. Proposition 3.4], choose $\gamma \in \Pi$ with $l\left(s_{\gamma} s_{\beta} s_{\gamma}\right)=l\left(s_{\beta}\right)-2$. and $\langle\gamma, \beta\rangle>0$. If $\gamma \in \Pi_{S \backslash I}$, then $\langle\beta, \gamma\rangle=\sum_{\alpha \in \Pi_{I}} c_{\alpha}\langle\alpha, \gamma\rangle \leq 0$, a contradiction. Hence $\gamma \in \Pi_{I}$. Since $\beta \in \Phi_{+}, \gamma \in \Pi$ and $\gamma \neq \beta$, we have $s_{\gamma} \beta \in \Phi_{+}$. Hence

$$
s_{\gamma}(\beta)=\beta-\left\langle\beta, \gamma^{\vee}\right\rangle \gamma \in \Phi_{+} \cap \mathbb{R} \Pi_{I}=\Phi_{+} \cap \mathbb{R}_{\geq 0} \Pi_{I} .
$$

By induction, $s_{\gamma}(\beta) \in W_{I} \Pi_{I}$ and so $\beta \in W_{I} \Pi_{I} \cap \Phi_{+}$, completing the inductive proof of (c). Part (b) follows easily from (c).

For (d), choose a reduced expression $w=s_{\alpha_{1}} \cdots s_{\alpha_{n}}$ for $w$ in $W$, with $\alpha_{i} \in \Pi$. We have, using Lemma 1.13 .

$$
w(k)-k=-\sum_{i=1}^{n}\left\langle k, \alpha_{i}^{\vee}\right\rangle \beta_{i}
$$

where $\beta_{i}:=s_{\alpha_{1}} \ldots s_{\alpha_{i-1}}\left(\alpha_{i}\right)$. Therefore

$$
0=\langle w k-k, a\rangle=-\sum_{i}\left\langle k, \alpha_{i}^{\vee}\right\rangle\left\langle\beta_{i}, a\right\rangle
$$

where $\left\langle k, \alpha_{i}\right\rangle \geq 0$, and $\left\langle\beta_{i}, a\right\rangle \geq 0$ since $\beta_{i} \in \Phi_{+}$. It follows that $\left\langle\beta_{i}, a\right\rangle=0$ for any $i$ with $\left\langle k, \alpha_{i}\right\rangle \neq 0$. If $\left\langle k, \alpha_{i}\right\rangle \neq 0$, then $\beta_{i} \in \mathbb{R}_{\geq 0} \Pi \cap a^{\perp}=\mathbb{R}_{\geq 0} \Pi_{I}$. Then by (b), $\beta_{i} \in W_{I} \Pi_{I} \cap \Phi_{+}$if $\left\langle k, \alpha_{i}\right\rangle \neq 0$ and so $s_{\beta_{i}} \in W_{I}$ for such $i$. Suppose that $\left\langle k, \alpha_{i}\right\rangle \neq 0$ for $i=i_{1}, \ldots, i_{m}$ where $1 \leq i_{1}<\ldots<i_{m} \leq n$ and that $\left\langle k, \alpha_{i}\right\rangle=0$ for $i \notin\left\{i_{1}, \ldots, i_{m}\right\}$. Then

$$
s_{\beta_{i_{1}}} \cdots s_{\beta_{i_{m}}} w(k)=s_{\alpha_{1}} \cdots \widehat{s}_{\alpha_{i_{i}}} \cdots \widehat{s}_{i_{m}} \cdots s_{\alpha_{n}}(k)=k
$$

where circumflexes denote factors omitted from the product, and therefore

$$
w(k)=s_{\beta_{i_{m}}} \cdots s_{\beta_{i_{1}}}(k) \in W_{I} k .
$$

2.5. Some of the results above hold for facial subgroups in general. Notably:

Corollary. Let $W^{\prime}$ be a facial subgroup of $W$, say $W^{\prime}=\operatorname{Stab}_{W}(q)$ where $q \in \mathscr{X}$. Then $\Phi_{W^{\prime},+}=W^{\prime} \Pi_{W^{\prime}} \cap \Phi_{+}=\mathbb{R}_{W^{\prime}} \cap \Phi_{+}=\mathbb{R}_{\geq 0} \Pi_{W^{\prime}} \cap \Phi=\Phi_{+} \cap q^{\perp}$.

Proof. Write $q=w p$ where $w \in W$ and $p \in \mathscr{C}$, so $W^{\prime}=w W_{I} w^{-1}$ where $W_{I}=$ $\operatorname{Stab}_{W}(p)$ is standard facial. Assume without loss of generality that $w \in W^{I}$. 
Hence

By 1.7. $\Pi_{W^{\prime}}=w \Pi_{I}$ and by Lemma 2.4(b), $\Phi_{I}=W_{I} \Pi_{I}=\mathbb{R}_{I} \cap \Phi=\Phi \cap p^{\perp}$.

$$
W^{\prime} \Pi_{W^{\prime}}=\Phi_{W^{\prime}}=w\left(\Phi_{I}\right)=\mathbb{R} w\left(\Pi_{I}\right) \cap w(\Phi)=\mathbb{R} \Pi_{W^{\prime}} \cap \Phi .
$$

Intersecting with $\Phi_{+}$gives the first two equalities in the statement of the corollary. The third holds since $\mathbb{R}_{\geq 0} \Pi_{W^{\prime}} \cap \Phi \subseteq \mathbb{R}_{W^{\prime}} \cap \Phi_{+}=\Phi_{W^{\prime},+} \subseteq \mathbb{R}_{\geq 0} \Pi_{W^{\prime}} \cap \Phi$. Lemma 1.10 (d) gives $\Phi_{W^{\prime}}=\Phi \cap q^{\perp}$ and intersecting with $\Phi_{+}$gives the remaining assertion.

2.6. Next we describe the intersections of reflection subgroups with (standard) facial subgroups.

Proposition. Let $W^{\prime}$ be a reflection subgroup of $W$.

(a) Let $J \subseteq S$ be facial. Then $W^{\prime \prime}:=W^{\prime} \cap W_{J}$ is a standard facial subgroup of $W^{\prime}$, with $\mathbb{R}_{\geq 0} \Pi_{W^{\prime \prime}}=\mathbb{R}_{\geq 0} \Pi_{W^{\prime}} \cap \mathbb{R}_{J}, \Pi_{W^{\prime \prime}}=\Pi_{W^{\prime}} \cap \Phi_{J}=\Pi_{W^{\prime}} \cap \mathbb{R}_{J}$, $\Phi_{W^{\prime \prime},+}=\Phi_{W^{\prime},+} \cap \Phi_{J}=\Phi_{W^{\prime},+} \cap \mathbb{R}_{J}$ and $\chi\left(W^{\prime \prime}\right)=\chi\left(W^{\prime}\right) \cap W_{J}$.

(b) If $W^{\prime \prime}$ is any facial subgroup of $W$, then $W^{\prime \prime \prime}:=W^{\prime} \cap W^{\prime \prime}$ is a facial subgroup of $W^{\prime}$ with $\Phi_{W^{\prime \prime \prime},+}=\Phi_{W^{\prime},+} \cap \Phi_{W^{\prime \prime}}=\Phi_{W^{\prime},+} \cap \mathbb{R}_{W^{\prime \prime}}$.

Proof. To prove (a), suppose that $W_{J}=W_{p}$ where $p \in \mathscr{C}$. Then $J=\Pi \cap p^{\perp}$. We have $p \in \mathscr{C}_{W^{\prime}}$ by 1.10 (e), so by 2.2(a) applied to $W^{\prime}, W^{\prime \prime}=W^{\prime} \cap W_{p}=\left(W^{\prime}\right)_{p}$ is a standard facial subgroup of $W^{\prime}$. By two applications of Lemma 2.4(a),

$$
\begin{aligned}
\mathbb{R}_{\geq 0} \Pi_{W^{\prime \prime}} & =\mathbb{R}_{\geq 0} \Pi_{W^{\prime}} \cap p^{\perp}=\mathbb{R}_{\geq 0} \Pi_{W^{\prime}} \cap\left(\mathbb{R}_{\geq 0} \Pi \cap p^{\perp}\right) \\
& =\mathbb{R}_{\geq 0} \Pi_{W^{\prime}} \cap\left(\mathbb{R}_{\geq 0} \Pi \cap \mathbb{R}_{J}\right)=\mathbb{R}_{\geq 0} \Pi_{W^{\prime}} \cap \mathbb{R} \Pi_{J} .
\end{aligned}
$$

Clearly, $\Pi_{W^{\prime \prime}} \subseteq \Pi_{W^{\prime}} \cap \Phi_{J} \subseteq \Pi_{W^{\prime}} \cap \mathbb{R}_{J}$ and $\Phi_{W^{\prime \prime},+} \subseteq \Phi_{W^{\prime},+} \cap \Phi_{J} \subseteq \Phi_{W^{\prime},+} \cap \mathbb{R} \Pi_{J}$. Intersecting $\mathbb{R}_{\geq 0} \Pi_{W^{\prime \prime}}=\mathbb{R}_{\geq 0} \Pi_{W^{\prime}} \cap \mathbb{R} \Pi_{J}$ with $\Pi_{W^{\prime \prime}}$ and $\Phi_{W^{\prime \prime},+}$ in turn shows that the above inclusions are actually equalities. Finally for (a), note that

$$
\chi\left(W^{\prime}\right)=\left\{s_{\alpha} \mid \alpha \in \Pi_{W^{\prime \prime}}\right\}=\left\{s_{\alpha} \mid \alpha \in \Pi_{W^{\prime}} \cap \Phi_{J}\right\}=\chi\left(W^{\prime}\right) \cap \Phi_{J} .
$$

For (b), write $W^{\prime \prime}=W_{q}$ for some $q \in \mathscr{X}$. By $1.10(\mathrm{e})$ and $2.2(\mathrm{~b}), W^{\prime \prime \prime}=$ $W^{\prime} \cap W_{q}=\left(W^{\prime}\right)_{q}$ is a facial subgroup of $W^{\prime}$ with $\Phi_{W^{\prime \prime \prime}}=\Phi_{W^{\prime}} \cap q^{\perp}$. The rest of (b) follows using Corollary 2.5

2.7. The preceding lemma implies that an intersection of two facial subgroups is a facial subgroup of both of them. The next lemma shows in particular that the intersections of two facial subgroups of $W$ is a facial subgroup of $W$.

Lemma. (a) The stabilizer of a point $x$ of $\mathscr{X}$ coincides with the pointwise stabilizer of the facet of $\mathscr{X}$ containing $x$.

(b) The pointwise stabilizer of a finite subset $Y$ of $\mathscr{X}$ is the stabilizer of some point in the convex hull of $Y$.

(c) The intersection of finitely many (standard) facial subgroups of $W$ is a (standard) facial subgroup of $W$.

Remarks. This would follow from the previous lemma if it was known that a (standard) facial subgroup of a (standard) facial subgroup of $W$ is a (standard) facial subgroup of $W$, but it seems possible that this fails in this generality (see 4.2(f) and Remark 2.11.

Proof. Part (a) follows from 2.3(b). For the proof of (b), denote the stabilizer in $W$ of $x \in \mathscr{X}$ as $W_{x}$. It suffices to show that if $x, y \in \mathscr{X}$, then $W_{x} \cap W_{y}=W_{z}$ for some $z$ in the closed segment $[x, y]=\{t x+(1-t) y \mid t \in \mathbb{R}, 0 \leq t \leq 1\}$ (cf. the proof of [34, 
Lemma 2.1.2]). We may assume $x \neq y$. The set $\Gamma:=\left\{\alpha \in \Phi_{+} \mid\langle x, \alpha\rangle\langle y, \alpha\rangle<0\right\}$ is finite by Lemma 1.10 (a). We may and do choose $z=t x+(1-t) y$ with $0<t<1$ in $[x, y]$ such that for all $\alpha \in \Gamma,\langle z, \alpha\rangle \neq 0$. Then for $\alpha \in \Phi_{+},\langle z, \alpha\rangle=0$ implies that $\langle\alpha, x\rangle=\langle\alpha, y\rangle=0$. By 1.10 (d), we get $W_{z} \subseteq W_{x} \cap W_{y}$. The reverse inclusion $W_{z} \supseteq W_{x} \cap W_{y}$ is clear, proving (b). Then (c) follows from (b) and 2.3(c) on recalling that $\mathscr{X}$ (and $\mathscr{C}$ ) is convex.

2.8. Slightly extending the terminology of 34 , define a subgroup $W^{\prime \prime}$ of $W$ to be a parabolic closure (resp., facial closure) of a subset $X$ of $W$ if $W^{\prime \prime}$ is a parabolic (resp., facial) subgroup $W$, and any parabolic (resp., facial) subgroup of $W$ containing $W^{\prime}$ contains $W^{\prime \prime}$. Such a parabolic (resp facial) closure exists if and only if the intersection of all parabolic (resp., facial) subgroups containing $X$ is parabolic (resp., facial), in which case it coincides with that intersection and so is unique. From the above, it follows that $X$ has a parabolic (resp., facial) closure if it is contained in some finite rank parabolic (resp., facial) subgroup of $W$; then the parabolic (resp., facial) closure is the parabolic (resp., facial) subgroup of minimal rank amongst those containing $X$. Similarly, we define the standard parabolic closure and standard facial closure of any subset of $W$. The standard parabolic closure of any subset exists, and the standard facial closure of a subset $X$ exists if $X$ is contained in a finite rank standard facial subgroup.

2.9. Well-known properties of finite subgroups of Coxeter groups and their parabolic closures are collected in the lemma below. For proofs, see for example [32, [4] and [34, 1.2.6, 3.2].

Lemma. Let $W^{\prime}$ be a reflection subgroup of $W$ and $W^{\prime \prime}$ be the reflection subgroup of $W$ with $\Phi_{W^{\prime \prime}}=\mathbb{R}_{W^{\prime}} \cap \Phi$.

(a) A subgroup of $W$ is finite if and only if it is contained in some finite parabolic subgroup of $W$.

(b) The following conditions (i)-(iii) are equivalent:

(i) $W^{\prime}$ is finite

(ii) $\Pi_{W^{\prime}}$ is finite and the "Cartan matrix" $(\langle\alpha, \beta\rangle)_{\alpha, \beta \in \Pi_{W^{\prime}}}$ is positive definite.

(iii) $\mathbb{R} \Pi_{W^{\prime}}$ is finite dimensional and the restriction of $\langle-,-\rangle$ to $\mathbb{R} \Pi_{W^{\prime}}$ is positive definite.

(c) If $W^{\prime}$ is finite, then $\Pi_{W^{\prime}}$ is linearly independent, $W^{\prime \prime}$ is finite, $W^{\prime \prime}$ has the same rank as $W^{\prime}$ and $W^{\prime \prime}$ is the parabolic closure of $W^{\prime}$. Further, every subset of $\Pi_{W^{\prime}}$ is facial in $\Pi_{W^{\prime}}$ (for the Coxeter group $W^{\prime}$ ).

Remarks. In particular, $\Pi_{W^{\prime}}$ is non-degenerate if $W^{\prime}$ is finite. In general, all parts of (c) fail without finiteness of $W^{\prime}$.

2.10. The next result gives information on the facial subsets of $S$. The main facts we require are (b) (which extends [49, Theorem 4] and [34, 2.2.4]) and (d) below.

Lemma. (a) Let $\tau \in \mathscr{C}$ and $I \subseteq S$ such that $W_{I}$ is finite. Let $\Delta$ denote the union of all components of $\Pi_{I}$ which are not contained in $\tau^{\perp}$. Then $\tau^{\prime}:=\sum_{w \in W_{I}} w \tau \in \mathscr{C}$ and $\tau^{\perp} \cap \Pi=\Pi_{I} \cup\left(\Pi \cap \tau^{\perp} \cap \Delta^{\perp}\right)$

(b) Let $I, J$ be subsets of $S$ such that $J$ is facial, $W_{I}$ is finite, and $I$ and $J$ are separated. Then $I \cup J$ is facial. In particular, if $\emptyset$ is facial, then $I$ is facial. 
(c) Let $I$ be a facial subset of $S$ and $K$ be a subset of $S$ such that $W_{K}$ is finite, $K$ is separated from $I \backslash K$, and each component of $K$ contains an element of $S \backslash I$. Then $I \backslash K$ is facial.

(d) If $I$ is a facial subset of $S$ and $J \subseteq S$ is $W$-conjugate to $I$, then $J$ is a facial subset of $S$.

Proof. First we prove (a). Let $J \subseteq S$ with $\Pi_{J}=\Lambda:=\Pi_{I} \cap \tau^{\perp}$. For $\alpha \in \Pi_{I}$, we have $s_{\alpha} \tau^{\prime}=\tau^{\prime}$ so $\left\langle\alpha, \tau^{\prime}\right\rangle=0$. Next, consider $\alpha \in \Pi \backslash \Pi_{I}$. We have $\left\langle\tau^{\prime}, \alpha\right\rangle=$ $\sum_{w \in W_{I}}\langle\tau, w \alpha\rangle$. Since $\alpha \in-\mathscr{C}_{I}$, we have $w(\alpha) \in \alpha+\mathbb{R}_{\geq 0} \Pi_{I}$ by 1.13 . In particular, $w(\alpha) \in \Phi_{+}$. Hence each term $\langle\tau, w \alpha\rangle \geq 0$, so it follows that $\left\langle\tau^{\prime}, \alpha\right\rangle \geq 0$. This shows that $\tau^{\prime} \in \mathscr{C}$. Further, $\left\langle\tau^{\prime}, \alpha\right\rangle=0$ if and only if $\langle\tau, \alpha\rangle=0$ and for each $w \in W_{I}, w(\alpha)-\alpha \in \mathbb{R}_{>0} \Pi_{I} \cap \tau^{\perp}=\mathbb{R}_{>0} \Lambda$. By Lemma 2.9. $\Lambda$ is facial in $\Pi_{I}$. Lemma 2.4 (c) and 1.13 therefore show that $w(\alpha)-\alpha \in \mathbb{R}_{>_{0}} \Lambda$ if and only if $w(\alpha)=w^{\prime}(\alpha)$ for some $w^{\prime} \in W_{J}$. But for $w^{\prime} \in W_{J}$, Lemma 1.10 implies that $w^{-1} w^{\prime} \in W_{I}$ stabilizes $\alpha \in-\mathscr{C}_{I}$ if and only if $w^{-1} w^{\prime} \in W_{I_{\alpha}}$ where $\Pi_{I_{\alpha}}=\Pi_{I} \cap \alpha^{\perp}$. It follows that (for $\alpha \in \Pi \backslash \Pi_{I}$ ), $\left\langle\tau^{\prime}, \alpha\right\rangle=0$ if and only if $\langle\tau, \alpha\rangle=0$ and $W_{I}=W_{J} W_{I_{\alpha}}$. By Lemma 1.17, however, $W_{I}=W_{J} W_{I_{\alpha}}$ if and only if every component of $\Pi_{I}$ which is not contained in $\Lambda$ (i.e. is not contained in $\tau^{\perp}$ ) is contained in $\Pi_{I_{\alpha}}$. That is, for $\alpha \in \Pi \backslash \Pi_{I}$, one has $\alpha \in\left(\tau^{\prime}\right)^{\perp}$ if and only if $\alpha \in \tau^{\perp} \cap \Delta^{\perp}$. Since it has already been noted that $\Pi_{I} \subseteq\left(\tau^{\prime}\right)^{\perp}$, this completes the proof of (a).

For (b), choose $\tau \in \mathscr{C}$ with $\tau^{\perp} \cap \Pi=\Pi_{J}$ and let $\tau^{\prime} \in \mathscr{C}$ and $\Delta$ be as in (a). We have $\Delta=\Pi_{I}$ since $\Pi_{I} \cap \tau^{\perp}=\Pi_{I} \cap \Pi_{J}=\emptyset$. Hence $\Pi \cap\left(\tau^{\prime}\right)^{\perp}=\Pi_{I} \cup\left(\Pi_{J} \cap \Pi_{I}^{\perp}\right)=\Pi_{I \cup J}$ by (a), since $\Pi_{I}$ and $\Pi_{J}$ are separated.

Next we prove (c). Let $\tau \in \mathscr{C}$ with $\Pi \cap \tau^{\perp}=\Pi_{I}$. Set $L:=I \backslash K$ and $J:=$ $I \cap K$. Note that $\Phi_{+} \backslash \Phi_{K \cup I}$ and $\Phi_{L,+}$ are $W_{K}$-invariant, and $\Phi_{I \cup K}=\Phi_{K} \cup \dot{\cup} \Phi_{L}$ since $I \cup K=K \cup \dot{\cup} L$ where $K$ and $L$ are separated. We have $\langle\tau, \alpha\rangle>0$ for all $\alpha \in \Phi_{+} \backslash \Phi_{I \cup K}$ and for all $\alpha \in \Phi_{K,+} \backslash \Phi_{J}$, while $\langle\tau, \alpha\rangle=0$ for all $\alpha \in \Phi_{L}$ and all $\alpha \in \Phi_{J}$. By 2.7(b), the set of points $\mu$ in the convex hull $Y$ of $\left\{w \tau \mid w \in W_{K}\right\}$ with stabilizer $W_{\mu}=\cap_{w \in W_{K}} W_{w \tau}$ is non-empty, and it is clearly $W_{K}$-stable. Since $V=\mathscr{X}_{K}=W_{K} \mathscr{C}_{K}$ by finiteness of $W_{K}$ and Lemma $1.10(\mathrm{~h})$, we may choose a point $\mu \in Y \cap \mathscr{C}_{K}$ such that $W_{\mu}=\cap_{w \in W_{K}} W_{w \tau}$. In particular, $\langle\mu, \alpha\rangle \geq 0$ for all $\alpha \in \Phi_{K,+}$. We have also $\langle\mu, \alpha\rangle>0$ for all $\alpha \in \Phi_{+} \backslash \Phi_{I \cup K}$ and $\langle\mu, \alpha\rangle=0$ for all $\alpha \in \Phi_{L}$ since $\mu$ is in the convex closure of $Y$. We now show that $\langle\mu, \alpha\rangle>0$ for $\alpha \in \Phi_{K,+}$, which will imply that $\mu \in \mathscr{C}$ and $\Pi \cap \mu^{\perp}=\Pi_{L}=\Pi_{I \backslash J}$ as required to prove (c). Note that by the assumptions and Lemma 1.16 applied to each component of $K$, each $W_{K}$-orbit on $\Phi_{K}$ contains a point of $\Phi_{K,+} \backslash \Phi_{J}$. Hence for $\alpha \in \Phi_{K,+}$ there is some $w \in W_{K}$ and $\beta \in \Phi_{K,+} \backslash \Phi_{J}$ with $w^{-1} \alpha=\beta$. We then have $\langle w \tau, \alpha\rangle=\langle\tau, \beta\rangle>0$, so $s_{\alpha} \notin W_{w \tau}, s_{\alpha} \notin W_{\mu}$ by definition of $\mu$, and hence $\langle\mu, \alpha\rangle \neq 0$. But we've already seen $\langle\mu, \alpha\rangle \geq 0$, so $\langle\mu, \alpha\rangle>0$ as required.

Finally we prove (d). Suppose that $w W_{I} w^{-1}=W_{J}$ where $I, J \subseteq S$ and $I$ is facial. Without loss of generality, we may assume that $w$ is a minimal length $\left(W_{J}, W_{I}\right)$-double coset representative. Then $w \Pi_{I}=\Pi_{J}$. Using induction, we may assume by 1.15 , that there exists $\alpha \in \Pi \backslash \Pi_{I}$ such that, setting $s=s_{\alpha} \in S$, the connected component $K$ of $I \cup\{s\}$ containing $s$ is of finite type and $w=w_{K} w_{K \backslash\{s\}}$. Let $t=w_{K} s w_{K}$. We have $\Pi_{I}=\Pi_{I \backslash K} \dot{\cup} \Pi_{K \backslash\{s\}}$ so

$$
\Pi_{J}=w \Pi_{I}=w\left(\Pi_{I \backslash K}\right) \dot{\cup} w\left(\Pi_{K \backslash\{s\}}\right)=\Pi_{I \backslash K} \dot{\cup} \Pi_{K \backslash\{t\}} .
$$

By (c), we conclude that $I \backslash K$ is facial. Since $K \backslash\{t\}$ is separated from $I \backslash K$ and is of finite type, we conclude from (b) that $J=(I \backslash K) \cup(K \backslash\{t\})$ is facial. 
2.11. The following is an immediate corollary of part (d) of the preceding lemma.

Corollary. The standard facial subgroups of $W$ are exactly the standard parabolic subgroups of $W$ which are facial.

2.12. The result below refines Lemma 2.7(c) and the well-known formula of Kilmoyer (see e.g. [34, 1.3.5(d)]) for the intersection of two parabolic subgroups of $W$.

Proposition. For any facial subsets $I, J \subseteq S$ and shortest $\left(W_{I}, W_{J}\right)$ double coset representative $d$, then

$$
W_{I} \cap d W_{J} d^{-1}=W_{I \cap d J d^{-1}}
$$

is a standard facial subgroup of $W$.

Proof. Let $W^{\prime}=W_{I} \cap d W_{J} d^{-1}$, which by 2.7(c) is a facial subgroup of $W$. Clearly, $d$ is of minimal length in its cosets $W_{I} d, d W_{J}$ and $W^{\prime} d$. By 1.6 and Proposition 2.6 (a) we have $\chi\left(d W_{J} d^{-1}\right)=d J d^{-1}, \chi\left(d^{-1} W_{I} d\right)=d^{-1} I d, \chi\left(W^{\prime}\right)=d J d^{-1} \cap W_{I}$, and

$$
d^{-1} \chi\left(W^{\prime}\right) d=\chi\left(d^{-1} W^{\prime} d\right)=\chi\left(d^{-1} W_{I} d \cap W_{J}\right)=d^{-1} I d \cap W_{J} .
$$

Hence $\chi\left(W^{\prime}\right)=\chi\left(W^{\prime}\right) \cap d \chi\left(d^{-1} W^{\prime} d\right) d^{-1}=I \cap d J d^{-1}$. Since $I \cap d J d^{-1} \subseteq S$, it follows that $W^{\prime}=W_{I \cap d J d^{-1}}$ is a facial standard parabolic subgroup, and hence a standard facial subgroup by Corollary 2.12 .

Remarks. Suppose that the standard facial subgroups and standard parabolic subgroups of $W$ coincide (see Remark 2.3. Then the Proposition specializes to Kilmoyer's formula and the argument above simplifies since $W_{I \cap d J d^{-1}}$ is obviously not just parabolic, but standard parabolic. This affords a simple proof of Kilmoyer's formula (not needing 2.11).

2.13. The proof of the following observation is left to the reader. See also Lemma 8.5 for similar results under stronger hypotheses.

Lemma. Suppose that $V=U \oplus U^{\prime}$ is an orthogonal direct sum decomposition of $V$ such that $\Pi=\Delta \dot{\cup} \Delta^{\prime}$ where $\Delta:=\Pi \cap U$ and $\Delta^{\prime}:=\Pi \cap U^{\prime}$. Then the facial subsets of $\Pi$ are precisely the unions of facial subsets of $\Delta$ (for the group $W_{\Delta}$ acting naturally either as reflection group on $V$ or on $U$ ) and the facial subsets of $\Delta^{\prime}$ (for $W_{\Delta^{\prime}}$ as reflection group on $V$ or on $\left.U^{\prime}\right)$.

Remarks. Let $\Delta$ be a non-empty finite subset of $\Pi$ which is separated from $\Delta^{\prime}:=$ $\Pi \backslash \Delta$ and such that that the restriction of $\langle-,-\rangle$ to $\mathbb{R} \Delta$ is non-singular. For example, $\Delta$ could be a finite union of finite type components of $\Pi$. Then the above applies with $U=\mathbb{R} \Delta, U^{\prime}=\Delta^{\perp}$ and $\Delta^{\prime}=\Pi \backslash \Delta$

2.14. Part (c) of the following was also obtained in [42].

Lemma. (a) The intersection of any directed (by reverse inclusion) family of reflection subgroups of $W$ is a reflection subgroup.

(b) The pointwise stabilizer of a subset $X$ of $\mathscr{X}$ is the reflection subgroup with root system $\Phi \cap X^{\perp}$.

(c) Any intersection of parabolic subgroups is a reflection subgroup.

Proof. Following [20, we observe that for any reflection subgroup $W^{\prime}$ of $W$ and $w \in W$, we have $w \in W^{\prime}$ if and only if there exist $n \in \mathbb{N}$ and reflections $t_{1}, \ldots, t_{n}$ of $T$ such that, setting $w_{i}=t_{i} \ldots t_{1}$ for $i=0,1, \ldots, n$ (so $w_{0}=1_{W}$ ), we have 
(i) $w_{n}=w$

(ii) $l\left(w_{0}\right)<l\left(w_{1}\right)<\ldots<l\left(w_{n}\right)$

(iii) $t_{i} \in W^{\prime} \cap T$ for all $i$.

In fact, $w \in W^{\prime}$ if and only if such $t_{i}$ exist with $t_{i} \in \chi\left(W^{\prime}\right)$ for all $i$; then $n=$ $l_{\left(W^{\prime}, \chi\left(W^{\prime}\right)\right)}(w)$.

Suppose that $D$ is a family of reflection subgroups of $D$ which is directed by reverse inclusion i.e. for $W^{\prime}, W^{\prime \prime}$ in $D$, there exists $W^{\prime \prime \prime} \in D$ with $W^{\prime \prime \prime} \subseteq W^{\prime} \cap W^{\prime \prime}$. Let $W^{\prime}:=\cap_{U \in D} U$. Now for fixed $w \in W$, there are only finitely many tuples $\left(n, t_{1}, \ldots, t_{n}\right)$ with $t_{i} \in T$ satisfying (i) and (ii) (since $n \leq l(w)$ and each $w_{i}$ lies in the (finite) Bruhat interval $[1, w]$ ). It follows that $w \in W^{\prime}$ if and only if there exists such a tuple $\left(n, t_{1}, \ldots, t_{n}\right)$ with $t_{i} \in U$ for all $i=1, \ldots, n$ and all $U \in D$. But then each $t_{i} \in W^{\prime} \cap T$ and $w=w_{n}=t_{n} \ldots t_{1} \in\left\langle W^{\prime} \cap T\right\rangle$. Hence $W^{\prime}$ is a reflection subgroup as required to prove (a). We observe that that the argument also shows that $w \in \chi\left(W^{\prime}\right)$ if and only if $l_{\left(W^{\prime}, \chi\left(W^{\prime}\right)\right.}(w)=1$ if and only if every tuple $\left(n, t_{1}, \ldots, t_{n}\right)$ as above has $n=1$ (in which case, such a tuple is unique).

Part (c) follows since the intersection of a family of parabolic subgroups is the intersection of the (directed by reverse inclusion) family of their finite intersections, and these finite intersections are (parabolic) reflection subgroups by Kilmoyer's formula. In (b), note that the pointwise stabilizer of $X$ is the intersection of the stabilizers of the points of $X$, which are parabolic (in fact, facial) subgroups. Hence the pointwise stabilizer is a reflection subgroup by (c). The remaining assertions are clear since a reflection $s_{\alpha}$ fixes $X$ pointwise if and only if it fixes each point of $X$ i.e. if and only if $\alpha$ is in $X^{\perp}$.

2.15. In 42, an example is given to show that an intersection of an arbitrary family of parabolic subgroups of an infinite rank Coxeter system need not be a parabolic subgroup. The following result gives a simple class of examples which illustrates the ubiquity of this phenomenon.

Proposition. Let $(W, S)$ be an infinite rank irreducible Coxeter system with locally finite Coxeter graph. Then there is a family $\left(W_{n}\right)_{n \in \mathbb{N}}$, of parabolic subgroups of $W$ such that $\cap_{n \in \mathbb{N}} W_{n}$ is a non-parabolic reflection subgroup with $|\mathbb{N}|$ components, which are all of type $A_{1}$.

Remarks. In the example in 42, the parabolic subgroups involved, and their intersection, are all irreducible of type $A_{\infty}$, which, together with $A_{\infty, \infty}$, is exceptional amongst all irreducible Coxeter groups in some respects (see [16]).

Proof. By Lemma 1.19, there is a subset $\Pi_{0}=\left\{\alpha_{0}, \alpha_{1}, \ldots\right\}$ of $\Pi$ such that for $i<j \in \mathbb{N},\left\langle\alpha_{i}, \alpha_{j}\right\rangle \neq 0$ if and only if $j=i+1$. Let $s_{i}:=s_{\alpha_{i}}$ for $i \in \mathbb{N}$. It is clear that there is a subset $J$ of $S$ with irreducible components $J_{i}$ for $i \in \mathbb{N}$ such that $\left|J_{i}\right|=i+1$. For example, one could take $J:=\cup_{i \in \mathbb{N}} J_{i}$ where $J_{i}:=$ $\left\{s_{2 i^{2}}, s_{2 i^{2}+1}, \ldots, s_{2 i^{2}+i}\right\}$. By Lemma 1.16, there is a reflection $t_{i} \in W_{J_{i}}$ of length $l\left(t_{i}\right)=2 i+1$. Let $K_{i}=\left\{t_{0}, t_{1}, \ldots, t_{i}\right\} \cup \bigcup_{j>i} J_{j}$. Since $\left\langle t_{k}\right\rangle$ is a parabolic subgroup of $W_{J_{k}}$ for $k \in \mathbb{N}$, it follows that $W_{K_{i}}$ is a parabolic subgroup of $W$ (with $\chi\left(W_{K_{i}}\right)=$ $K_{i}$ as its set of canonical generators). Let $W^{\prime}=\left\langle t_{0}, t_{1}, \ldots\right\rangle$, which is a reflection subgroup of $W$ with $\chi\left(W^{\prime}\right)=R:=\left\{t_{0}, t_{1}, \ldots\right\}$ as canonical generators (since $R$ is both the set of all reflections of $W^{\prime}$ and a minimal generating set of $W^{\prime}$ ). Obviously, $W^{\prime}$ has $|\mathbb{N}|$ components, all of type $A_{1}$. We claim that $\cap_{i \in \mathbb{N}} W_{K_{i}}=W^{\prime}$. Clearly, $W^{\prime} \subseteq \cap W_{K_{i}}$ since $t_{j} \in W_{K_{i}}$ for all $j, i \in \mathbb{N}$. For the reverse inclusion, let $w \in \cap W_{K_{i}}$. 
Set $L_{n}:=J_{1} \cup \ldots \cup J_{n}$. Note that $w \in W_{J}=\cup_{n \in \mathbb{N}} W_{L_{n}}$ so $w \in W_{L_{n}}$ for some $n \in \mathbb{N}$. Hence $w \in W_{L_{n}} \cap W_{K_{n}}=\left\langle\left\{t_{0}, \ldots, t_{n}\right\}\right\rangle \subseteq W^{\prime}$ as claimed.

To complete the proof, it suffices to show that $R$ is not $W$-conjugate to any subset of $S$. But since $\{l(t) \mid t \in R\}$ is not bounded above, it follows that for any $u \in W,\left\{l\left(u t u^{-1}\right) \mid t \in R\right\}$ is also not bounded above (since $l\left(u t u^{-1}\right) \geq l(t)-2 l(u)$ ) and so $u R u^{-1} \nsubseteq S$.

2.16. Weakly facial subgroups and locally parabolic subgroups. Call a (reflection) subgroup of $W$ weakly facial if it is an intersection of (possibly infinitely many) facial subgroups, or equivalently, if it is the pointwise stabilizer of a subset of the Tits cone. There is a natural Galois connection between the subsets of $\mathscr{X}$ and subsets of $W$ (cf. [34, 2.1]) with the weakly facial subgroups as the stable subsets of $W$. Also, the set of weakly facial subgroups forms a complete lattice when ordered by inclusion. Any subset $X$ of $W$ has a weakly facial closure, defined to be the weakly facial subgroup obtained by intersecting all (weakly) facial subgroups containing $X$. If $W$ is finitely generated, the set of weakly facial subgroups is equal to the set of facial subgroups. Some of the results on facial subgroups have analogues for weakly facial subgroups; for example, intersections of weakly facial subgroups are weakly facial, and (using $1.10(\mathrm{e})$ ) the intersection of a weakly facial subgroup of $W$ with a reflection subgroup $W^{\prime}$ of $W$ is a weakly facial subgroup of $W^{\prime}$.

In [42, a reflection subgroup $W^{\prime}$ of $W$ is defined to be a locally parabolic subgroup if every finite subset of its canonical generators $\chi\left(W^{\prime}\right)$ is $W$-conjugate to a subset of $S$. It is shown in op. cit. that parabolic subgroups are locally parabolic, and that arbitrary intersections of locally parabolic (e.g. parabolic) subgroups are locally parabolic. Also, if $S$ is finite, the notions of parabolic and locally parabolic subgroups of $W$ coincide.

In particular, weakly facial subgroups are locally parabolic, and if $S$ is finite and all parabolic subgroups are facial, the notions of parabolic, weakly facial and locally parabolic subgroups coincide. It is not known for infinite $S$ whether every locally parabolic subgroup is equal to some intersection of parabolic subgroups (or equivalently, if the weakly facial and locally parabolic subgroups coincide when every parabolic subgroup is facial). We also leave open the question as to whether there is a natural analogue of the notion of locally parabolic subgroups, reducing to the facial subgroups for $W$ of finite rank, in the general situation in which not all parabolic subgroups are facial.

2.17. Parabolic closure of union of two special parabolic subgroups. The following result extends to (possibly infinite rank) Coxeter groups a result established for Weyl groups of Kac-Moody Lie algebras in [41, Theorem 4(b)].

Proposition. Let $(W, S)$ be any Coxeter system.

(a) Suppose that $J, K \subseteq S$ are special and $d$ is of minimal length in its double coset $W_{J \cup J^{\perp}} d W_{K \cup K^{\perp}}$. Then the parabolic closure of $W_{J} \cup d W_{K} w^{-1}$ exists and is equal to the standard parabolic closure $W_{L}$ of $J \cup\{d\} \cup K$.

(b) In (a), $W_{L}$ is special.

(c) The union of a finite family of special parabolic subgroups has a parabolic closure, which is itself a special parabolic subgroup.

Remarks. We do not know if, in arbitrary $W$, the union of finitely many parabolic subgroups necessarily has a parabolic closure. Proposition 2.15implies that in any 
infinite rank irreducible Coxeter group with locally finite Coxeter graph, there is a denumerable family of parabolic subgroups (all of type $A_{1}$ ) for which their union does not have a parabolic closure.

Proof. By definition of standard parabolic closure, in part (a) one has $L:=J \cup K \cup$ $\left\{s_{1}, \ldots, s_{n}\right\}$ for some (and every) reduced expression $d=s_{1} \ldots s_{n}$. Clearly, $W_{J} \cup$ $d W_{K} d^{-1} \subseteq W_{L}$. To show in (a) that $W_{L}$ is the desired parabolic closure of $W_{J} \cup$ $d W_{K} w^{-1}$, it will suffice to show that if $u \in W$ and $M \subseteq S$ with $W_{J} \cup d W_{K} w^{-1} \subseteq$ $u W_{M} u^{-1}$, then $W_{L} \subseteq u W_{M} u^{-1}$. For this, replace $u$ by the minimal length element of its coset $u W_{M}$ to assume without loss of generality that $u \in W^{M}$. Write $u=u_{0} u_{1}$ where $u_{0} \in W_{J^{\perp} \cap d K^{\perp} d^{-1}}$ and $u_{1}^{-1} \in W^{J^{\perp} \cap d K^{\perp} d^{-1}}$. Since conjugations by elements of $W_{J \perp} \cap d K^{\perp} d^{-1}$ fix $W_{J} \cup d W_{K} d^{-1}$ pointwise, $W_{J} \cup d W_{K} w^{-1} \subseteq u_{1} W_{M} u_{1}^{-1}$. Now $l(u)=l\left(u_{0}\right)+l\left(u_{1}\right)$, so $u_{1} \in W^{M}$. This implies that $\Pi_{J} \subseteq u_{1} \Pi_{M}$, by 1.7. Hence $u_{1}^{-1} \Pi_{J} \subseteq \Pi_{M}$. Since all irreducible components of $\Pi_{J}$ are infinite, it follows by Remark 1.15 that $u_{1} \in W_{J^{\perp}}$ and $\Pi_{J} \subseteq \Pi_{M}$ i.e. $J \subseteq M$.

Now we also have $W_{K} \subseteq d^{-1} u_{1} W_{M} u_{1}^{-1} d$. Write $\bar{d}^{-1} u_{1}=x w$ where $x \in W^{M}$ and $w \in W_{M}$, so $W_{K} \subseteq x W_{M} x^{-1}$. A similar argument to that just above shows that $\Pi_{K} \subseteq x \Pi_{M}, x \in W_{K^{\perp}}$ and $K \subseteq M$. Now we have $w=x^{-1} d^{-1} u_{1} \in W_{M}$ where $x^{-1} \in W_{K^{\perp}}, u_{1}^{-1} \in W_{J^{\perp}}^{J^{\perp}} \cap d K^{\perp} d^{-1}$ and $d^{-1} \in K^{\perp} W^{J^{\perp}}$ (actually, (a)-(b) only require this condition on $d$, not the stronger assumed condition $d^{-1} \in K \cup K^{\perp} W^{J \cup J^{\perp}}$, but the two formulations are easily equivalent). By [9, Proposition 2.7] (for general Coxeter groups), one has $l(w)=l\left(x^{-1}\right)+l\left(d^{-1}\right)+l\left(u_{1}\right)$. Since $w \in W_{M}$, one has $x, d, u_{1} \in W_{M}$. Hence $J \cup\{d\} \cup K \subseteq W_{M}$ and therefore $L \subseteq M$. In case $W$ is of finite rank, we can shortcut the rest of the proof of (a) at this point by noting that the above already shows that $W_{L}$ is a parabolic subgroup of minimal rank containing $W_{J} \cup d W_{K} w^{-1}$ and hence it is the parabolic closure as desired.

We continue the proof of (a) in general. The above shows that $u_{1} \in W^{M} \cap W_{M}=$ $\{1\}$ so $u=u_{0} \in W_{J^{\perp} \cap d K^{\perp} d^{-1}}$. Let $u=r_{k} \cdots r_{1}, r_{i} \in J^{\perp} \cap d K^{\perp} d^{-1}$, be a reduced expression for $u$. Note $u \in W^{M} \subseteq W^{L}$. We claim that $r_{1}, \ldots, r_{k} \in L^{\perp}$ so $u \in W_{L^{\perp}}$. This would imply $u W_{M} u^{-1} \supseteq u W_{L} u^{-1}=W_{L}$, completing the proof of (a). To prove the claim, it suffices to show that if $r_{1}, \ldots, r_{i-1} \in L^{\perp}$ where $1 \leq i \leq k$, then $r_{i} \in L^{\perp}$. The hypothesis and $u \in W^{L}$ imply that $r_{k} \cdots r_{i} \in W^{L}$ and so $r_{i} \notin L$. We have by definition $r_{i} \in J^{\perp}$ and $r_{i} d=d r_{i}^{\prime}$ where $r_{i}^{\prime} \in K^{\perp}$. Note $d \in W_{L}$ and $r_{i} \notin W_{L}$, so $\left\{\alpha_{r_{i}^{\prime}}\right\}=d^{-1}\left\{\alpha_{r_{i}}\right\}$ and applying Theorem 1.15 shows that $d$ has a reduced expression involving only simple reflections in $r_{i}^{+} \subseteq S \backslash\left\{r_{i}\right\}$. In particular, $r_{i}^{\prime}=r_{i}$, which gives $K \subseteq r_{i}^{\perp}$. This shows that $W_{r_{i}^{\perp}} \supseteq J \cup K \cup\{d\}$ so $L \subseteq r_{i}^{\perp}$ and $r_{i} \in L^{\perp}$. This proves the claim and hence (a).

For the proofs of (b)-(c), consider first two parabolic subgroups $a W_{J} a^{-1}$ and $b W_{K} b^{-1}$ where $a, b \in W$ and $J, K \subseteq S$ be special. Write $a^{-1} b=p d q$ where $p \in W_{J \cup J^{\perp}}, q \in W_{K \cup K^{\perp}}$ and $d$ is the shortest length double coset representative in $W_{J \cup J^{\perp}} a^{-1} b W_{K \cup K^{\perp}}$. Then

$$
a W_{J} a^{-1} \cup b W_{J} b^{-1}=a\left(W_{J} \cup p d q W_{K}(p d q)^{-1}\right) a^{-1}=a p\left(W_{J} \cup d W_{K} d^{-1}\right)(a p)^{-1} .
$$

Letting $W_{L}$ be the parabolic closure of $W_{J} \cup d W_{K} d^{-1}$, with $L$ as in (a), we see that $a W_{J} a^{-1} \cup b W_{K} b^{-1}$ has a parabolic closure $a p W_{L}(a p)^{-1}$. Also, if (b) holds then $a p W_{L}(a p)^{-1}$ is special. Hence we need only prove (b) (for then (c) follows by induction on the number of parabolic subgroups).

Finally we prove (b) (i.e. that $W_{L}$ is special in (a)) by induction on $l(d)$. If $l(d)=0$, then $L=J \cup K$ is clearly special since any component of $J \cup K$ contains 
either a component of $J$ or one of $K$, and hence is of infinite type because $J$ and $K$ are special. Suppose $l(d)>0$ and write $d=s f$ where $s \in L$ and $l(f)<l(d)$. Since $d^{-1} \in W^{J \cup J^{\perp}}$, we have $s \notin J \cup J^{\perp}$. Therefore, $H:=J \cup\{s\}$ is connected, necessarily of infinite type since it contains $J$. Since $s \in L$, the parabolic closure of $W_{H} \cup d W_{K} d^{-1}$ clearly exists and is clearly to $W_{L}$ also. Let $e$ be the minimal length double coset representative in $W_{H \cup H^{\perp}} d W_{K \cup K^{\perp}}$. From above, $W_{L}$ is $W$-conjugate to the parabolic closure $W_{L^{\prime}}$ of $W_{H} \cup e W_{K} e^{-1}$. Since $f=s d$ is in the above double coset, $l(e) \leq l(f)<l(d)$. By induction, $W_{L^{\prime}}$ is special and hence its conjugate $W_{L}$ is special too (in fact, $L^{\prime}=L$ by 1.15 , though we don't need this fact).

\section{The IMAGinary CONE}

3.1. For any reflection subgroup $W^{\prime}$ of $W$, define the $W^{\prime}$-invariant cone

$$
\mathscr{Y}_{W^{\prime}}:=\bigcap_{w \in W^{\prime}} w\left(\mathbb{R}_{\geq 0} \Pi_{W^{\prime}}\right)
$$

i.e. $\mathscr{Y}_{W^{\prime}}$ is the set of all $v \in V$ such that for each $w \in W^{\prime}, w(v)$ is expressible as a non-negative linear combination of positive roots $\alpha \in \Pi_{W^{\prime}}$.

For any subset $X$ of $V$, let $X^{*}:=\left\{v \in V \mid\langle v, X\rangle \subseteq \mathbb{R}_{\geq 0}\right\}$ (see Appendix A).

Lemma. Let $W^{\prime}$ be any reflection subgroup of $W$. Then

(a) $\mathscr{Y}_{W^{\prime}} \subseteq \mathscr{Y}_{W}$.

(b) For any $w \in W, \mathscr{Y}_{w W^{\prime} w^{-1}}=w \mathscr{Y}_{W^{\prime}}$.

(c) If $W^{\prime}$ is facial, then $\mathscr{Y}_{W^{\prime}}=\mathscr{Y}_{W} \cap \mathbb{R} \Pi_{W^{\prime}}$.

(d) If $\Pi_{W^{\prime}}$ is finite and $\langle-,-\rangle$ is non-singular, then $\mathbb{R}_{\geq 0} \Pi_{W^{\prime}}=\mathscr{C}_{W^{\prime}}^{*}$ and $\mathscr{Y}_{W^{\prime}}=\mathscr{X}_{W^{\prime}}^{*}$.

Remarks. Even if $\langle-,-\rangle$ is non-singular, $V$ is finite dimensional and $\Pi$ is finite, the cone $\mathbb{R}_{\geq 0} \Pi_{W^{\prime}}$ need not be closed if $\chi\left(W^{\prime}\right)$ is infinite.

Proof. Suppose that $v \in \mathscr{Y}_{W^{\prime}}$. For (a), we have to show that for $w \in W, w(v) \in$ $\mathbb{R}_{\geq 0} \Phi_{+}$. Write $w=w^{\prime} w^{\prime \prime}$ where $w^{\prime \prime} \in W^{\prime}$ and $N\left(w^{\prime-1}\right) \cap W^{\prime}=\emptyset$. Since $v \in \mathscr{Y}_{W^{\prime}}$, we have $w^{\prime \prime}(v) \in \mathbb{R}_{\geq 0} \Pi_{W^{\prime}}$. But for $\alpha \in \Pi_{W^{\prime}}$, we have $w^{\prime}(\alpha) \in \Phi_{+} \subseteq \mathbb{R}_{\geq 0} \Pi$ so $w(v)=w^{\prime}\left(w^{\prime \prime}(v)\right) \in \mathbb{R}_{\geq 0} \Pi$ as required for (a). Part (b) follows readily from the definitions on noting that $\Pi_{w W^{\prime} w^{-1}}=x \Pi_{W^{\prime}}$ where $x$ is the element of minimal length in the coset $w W^{\prime}$.

To prove (c), write $W^{\prime}=w W_{I} w^{-1}$ where $W_{I}$ is standard facial and $w$ is of minimal length in $w W_{I}$. By (a) and the definitions,

$$
\mathscr{Y}_{W_{I}} \subseteq \mathscr{Y}_{W} \cap \mathbb{R}_{\geq 0} \Pi_{I} \subseteq \mathscr{Y}_{W} \cap \mathbb{R}_{I} \subseteq \mathbb{R}_{\geq 0} \Pi \cap \mathbb{R}_{I}=\mathbb{R}_{\geq 0} \Pi_{I}
$$

where the last equality uses Lemma 2.4(a). Since $\mathscr{Y}_{W}$ and $\mathbb{R}_{I}$ are $W_{I}$-invariant, so is $\mathscr{Y}_{W} \cap \mathbb{R}_{I}$ and therefore $\mathscr{Y}_{W} \cap \mathbb{R}_{I} \subseteq \cap_{w \in W_{I}} w\left(\mathbb{R}_{\geq 0} \Pi_{I}\right)=\mathscr{Y}_{W_{I}}$. Hence $\mathscr{Y}_{W_{I}}=\mathscr{Y} \cap \mathbb{R} \Pi_{I}$. Finally by (b) and 1.61 .7 .

$$
\mathscr{Y}_{W^{\prime}}=w \mathscr{Y}_{W_{I}}=w\left(\mathscr{Y}_{W} \cap \mathbb{R} \Pi_{I}\right)=w\left(\mathscr{Y}_{W}\right) \cap \mathbb{R} w\left(\Pi_{I}\right)=\mathscr{Y}_{W} \cap \mathbb{R} \Pi_{W^{\prime}},
$$

completing the proof of (c).

For $(\mathrm{d})$, note that if $\Pi_{W^{\prime}}$ is finite and $\langle-,-\rangle$ is non-singular on $V$, then one has $\left(\mathbb{R}_{\geq 0} \Pi_{W^{\prime}}\right)^{*}=\mathscr{C}_{W^{\prime}}$ by definition and so $\mathbb{R}_{\geq 0} \Pi_{W^{\prime}}=\mathscr{C}_{W^{\prime}}^{*}$ by the duality theorem for polyhedral cones (see Lemma A.10. This implies that

$$
\mathscr{Y}_{W^{\prime}}=\bigcap_{w \in W^{\prime}} w\left(\mathbb{R}_{\geq 0} \Pi_{W^{\prime}}\right)=\bigcap_{w \in W^{\prime}} w\left(\mathscr{C}_{W^{\prime}}^{*}\right)=\left(\bigcup_{w \in W^{\prime}} w\left(\mathscr{C}_{W^{\prime}}\right)\right)^{*}=\mathscr{X}_{W^{\prime}}^{*}
$$


is the dual cone of $\mathscr{X}_{W^{\prime}}$.

3.2. We now define a $W$-stable cone $\mathscr{Z}$ called the imaginary cone of $W$ on $V$ (see [33, $§ 5.3-5.4,5.8]$ and 10.1) which is the basic object of study in this paper. It is a $W$-stable subset of $\mathscr{Y}$ defined in terms of a natural fundamental domain $\mathscr{K}$. It will eventually be shown that, under mild finiteness conditions, $\mathscr{Z}$ contains the interior of $\mathscr{Y}$ and satisfies analogues of the properties of $\mathscr{Y}$ stated in Lemma 3.1

Define $\mathscr{K}=\mathscr{K}_{W}:=\mathbb{R}_{\geq 0} \Pi_{W} \cap-\mathscr{C}_{W}$ and $\mathscr{Z}=\mathscr{Z}_{W}:=\cup_{w \in W} w\left(\mathscr{K}_{W}\right)$. Note that any $W$-orbit on $\mathscr{Z}$ contains a unique point of $\mathscr{K}$, since any $W$-orbit on $\mathscr{X}$ contains a unique point of $\mathscr{C}$.

Proposition. (a) $\mathscr{Z}=\mathscr{Y} \cap-\mathscr{X}$.

(b) $\mathscr{K}$ and $\mathscr{Z}$ are cones.

(c) If $z, z^{\prime} \in \mathscr{Z}$, then $\left\langle z, z^{\prime}\right\rangle \leq 0$.

(d) Let $W_{i}$, for $i \in I$, denote the irreducible components of $(W, S)$. Then $\mathscr{K}_{W}=\sum_{i} \mathscr{K}_{W_{i}}$ and $\mathscr{Z}_{W}=\sum_{i} \mathscr{Z}_{W_{i}}$.

(e) For any reflection subgroup $W^{\prime}$ of $W$ and any $w \in W, \mathscr{Z}_{w W^{\prime} w^{-1}}=w \mathscr{Z}_{W^{\prime}}$. If also $w$ is the element of minimal length in $w W^{\prime}$, then $\mathscr{K}_{w W^{\prime} w^{-1}}=w \mathscr{K}_{W^{\prime}}$.

Proof. By 1.13 , if $k \in \mathscr{K}$ and $w \in W$, then

$$
w k=(w k-k)+k \in \mathbb{R}_{\geq 0} \Pi+\mathbb{R}_{\geq 0} \Pi \subseteq \mathbb{R}_{\geq 0} \Pi .
$$

Thus, $\mathscr{Z} \subseteq \mathbb{R}_{\geq 0} \Pi$ and since $\mathscr{Z}$ is $W$-stable, $\mathscr{Z} \subseteq \mathscr{Y}$. Also, since $\mathscr{K} \subseteq-\mathscr{C}$, we have $\mathscr{Z}=W \mathscr{K} \subseteq W(-\mathscr{C})=-\mathscr{X}$. This proves that $\mathscr{Z} \subseteq \mathscr{Y} \cap-\mathscr{X}$. On the other hand, let $z \in \mathscr{Y} \cap-\mathscr{X}$. Since $z \in-\mathscr{X}$, there is some $w \in W$ with $w^{-1} z \in-\mathscr{C}$. Since $z \in \mathscr{Y}$, we have $w^{-1} z \in \mathscr{Y} \subseteq \mathbb{R}_{\geq 0} \Pi$ and so $w^{-1} z \in \mathbb{R}_{\geq 0} \Pi \cap-\mathscr{C}=\mathscr{K}$. Hence $z=w\left(w^{-1} z\right) \in W \mathscr{K}=\mathscr{Z}$. This shows $\mathscr{Z} \supseteq \mathscr{Y} \cap-\mathscr{X}$ and proves (a).

Part (b) follows from the definitions and (a), using that $\mathbb{R}_{\geq 0} \Pi, \mathscr{C}, \mathscr{X}$ and $\mathscr{Y}$ are cones and that an intersection of cones is a cone.

To prove (c), choose $w \in W$ with $w z \in \mathscr{K}$. Then $w z \in-\mathscr{C}$ and $w z^{\prime} \in \mathscr{Z} \subseteq$ $\mathbb{R}_{>0} \Pi$, so

$$
\left\langle z, z^{\prime}\right\rangle=\left\langle w z, w z^{\prime}\right\rangle \in\left\langle\mathbb{R}_{\geq 0} \Pi,-\mathscr{C}\right\rangle \subseteq \mathbb{R}_{\leq 0} .
$$

Now we prove (d). If $\alpha \in \Pi_{i}:=\Pi_{W_{i}}$, then $\langle\alpha, \beta\rangle=0$ for all $\beta \in \Pi \backslash \Pi_{i}$. This implies that $\mathbb{R}_{\geq 0} \Pi_{i} \cap-\mathscr{C}_{i}=\mathbb{R}_{\geq 0} \Pi_{i} \cap-\mathscr{C}$ where $\mathscr{C}_{i}:=\mathscr{C}_{W_{i}}$. Hence $\mathscr{K}_{i}:=\mathscr{K}_{W_{i}} \subseteq \mathscr{K}$ and $\sum_{i} \mathscr{K}_{i} \subseteq \mathscr{K}$ by (b). We also get $\mathscr{Z}_{i}:=W_{i} \mathscr{K}_{i} \subseteq W \mathscr{K}=\mathscr{Z}$ and $\sum_{i} \mathscr{Z}_{i} \subseteq \mathscr{Z}$.

For the other direction, let $k \in \mathscr{K} \subseteq \mathbb{R}_{\geq 0} \Pi$. We may write $k=\sum_{i} k_{i}$ where $k_{i} \in \mathbb{R}_{\geq 0} \Pi_{i}$ and almost all $k_{i}=0$. For $\alpha \in \Pi_{i}$ we have $\left\langle\alpha, k_{i}\right\rangle=\langle\alpha, k\rangle \leq 0$ and so $\bar{k}_{i} \in \mathscr{K}_{i}$. So $k \in \sum_{i} \mathscr{K}_{i}$. If $z \in \mathscr{Z}$, choose $w \in W$ with $w^{-1} z \in \mathscr{K}$, say $w^{-1}(z)=\sum_{i} k_{i}$ with all $k_{i} \in \mathscr{K}_{i}$ and almost all $k_{i}=0$. Also, write $w=\prod_{i \in I} w_{i}$ with $w_{i} \in W_{i}$, almost all $w_{i}=1$. We have $w k_{i}=w_{i} k_{i} \in \mathscr{Z}_{i}$ and

$$
z=w\left(w^{-1} z\right)=\sum_{i} w k_{i}=\sum_{i} w_{i} k_{i} \in \sum_{i} \mathscr{Z}_{i}
$$

as required for (d). Finally, the first part of (e) follows from (a), $1.10(\mathrm{~g})$ and 3.1 (b) and the second follows from Lemma 1.11 (a) and 1.6 1.7.

3.3. For $I \subseteq S$, abbreviate $\mathscr{Y}_{I}:=\mathscr{Y}_{W_{I}} \mathscr{K}_{I}:=\mathscr{K}_{W_{I}}, \mathscr{Z}_{I}=\mathscr{Z}_{W_{I}}$ etc.

Lemma. (a) If $I \subseteq S$, then $\mathscr{K}_{I} \subseteq \mathscr{K}$ and $\mathscr{Z}_{I} \subseteq \mathscr{Z}$.

(b) $\mathscr{K}=\cup_{I} \mathscr{K}_{I}$ and $\mathscr{Z}=\cup_{I} \mathscr{Z}_{I}$ where in both unions, I ranges over the finite subsets of $S$. 
MATTHEW J. DYER

Proof. First we prove (a). Let $k \in \mathscr{K}_{I}$. Then $k \in \mathbb{R}_{\geq 0} \Pi_{I}$ and $\left\langle k, \Pi_{I}\right\rangle \subseteq \mathbb{R}_{\leq 0}$. Since $\Pi_{I} \subseteq \Pi$ and $\left\langle\Pi_{I}, \Pi_{S \backslash I}\right\rangle \subseteq \mathbb{R}_{\leq 0}$, we get $k \in \mathbb{R}_{\geq 0} \Pi$ and $\langle k, \Pi\rangle \subseteq \mathbb{R}_{\leq 0}$ i.e. $k \in \mathscr{K}$. Hence $\mathscr{K}_{I} \subseteq \mathscr{K}$, and therefore $\mathscr{Z}_{I}=W_{I} \mathscr{K}_{I} \subseteq W \mathscr{K}=\mathscr{Z}$ proving (a).

To prove (b), let $z \in \mathscr{Z}$. Choose $w \in W$ such that $w z=k \in \mathscr{K}$. We may choose a finite subset $I$ of $S$ such that both $w \in W_{I}$ and $k \in \mathbb{R}_{\geq 0} \Pi_{I}$. Clearly, $\left\langle k, \Pi_{I}\right\rangle \subseteq\langle k, \Pi\rangle \subseteq \mathbb{R}_{\leq 0}$ so $k \in \mathscr{K}_{I}$ and $z=w^{-1} k \in W_{I} \mathscr{K}_{I}=\mathscr{Z}_{I}$. This shows that $\mathscr{Z} \subseteq \cup_{I} \mathscr{Z}_{I}$ with union over finite $I \subseteq S$, and the reverse inclusion holds by (a). Now if $z \in \mathscr{K}$, we may take $w=1$ in the above argument to see that $z=k \in \mathscr{K}_{I}$, hence $\mathscr{K} \subseteq \cup_{I} \mathscr{K}_{I}$ with union over finite $I \subseteq S$, and the reverse inclusion holds by (a) again.

3.4. In the case of a facial subset $I$ of $S$, there is the following stronger connection between imaginary cones of $W$ and $W_{I}$.

Lemma. Let I be a facial subset of $S$. Then

(a) $\mathscr{K}_{I}=\mathscr{K} \cap \mathbb{R} \Pi_{I}=\mathscr{K} \cap \mathbb{R}_{\geq 0} \Pi_{I}$.

(b) $\mathscr{Z}_{I}=\mathscr{Z} \cap \mathbb{R} \Pi_{I}=\mathscr{Z} \cap \mathbb{R}_{\geq 0} \Pi_{I}$.

Proof. It is enough to prove the first equality in each of (a)-(b), since $\mathscr{K}_{I}, \mathscr{Z}_{I} \subseteq$ $\mathbb{R}_{\geq 0} \Pi_{I}$. For (a), note that by Lemma 2.4 .

$$
\begin{aligned}
\mathscr{K} \cap \mathbb{R} \Pi_{I} & =\left\{v \in \mathbb{R}_{\geq 0} \Pi \mid\langle v, \alpha\rangle \leq 0 \text { for all } \alpha \in \Pi\right\} \cap \mathbb{R}_{I} \\
& =\left\{v \in \mathbb{R}_{\geq 0} \Pi_{I} \mid\langle v, \alpha\rangle \leq 0 \text { for all } \alpha \in \Pi\right\} \\
& =\left\{v \in \mathbb{R}_{\geq 0} \Pi_{I} \mid\langle v, \alpha\rangle \leq 0 \text { for all } \alpha \in \Pi_{I}\right\}=\mathscr{K}_{I}
\end{aligned}
$$

since for $v \in \mathbb{R}_{\geq 0} \Pi_{I}, \alpha \in \Pi_{S \backslash I}$ we have $\langle v, \alpha\rangle \leq 0$.

For (b), note first that using (a), we have

$$
\mathscr{Z}_{I}=\bigcup_{w \in W_{I}} w\left(\mathscr{K}_{I}\right) \subseteq \mathbb{R} \Pi_{I} \cap \bigcup_{w \in W} w(\mathscr{K})=\mathscr{Z} \cap \mathbb{R} \Pi_{I} .
$$

Conversely, suppose that $z \in \mathscr{Z} \cap \mathbb{R} \Pi_{I}$. Write $z=w k$ where $w \in W$ and $k \in \mathscr{K}$. We have $z=(w k-k)+k$ where $k$ and $w k-k$ are in $\mathbb{R}_{\geq 0} \Pi$, using 1.13 Since $z \in \mathbb{R}_{\geq 0} \Pi \cap \mathbb{R}_{I}=\mathbb{R}_{\geq 0} \Pi_{I}$ and $\Pi_{I}$ is facial, it follows that $k, w k-k \in \mathbb{R}_{\geq 0} \Pi_{I}$. By 2.4. $w k=w^{\prime} k$ for some $w^{\prime} \in W_{I}$. Hence $k \in \mathscr{K} \cap \mathbb{R}_{\geq 0} \Pi_{I}=\mathscr{K}_{I}$ and $z=w k=$ $w^{\prime} k \in W_{I} \mathscr{K}_{I}=\mathscr{Z}_{I}$ as required for (b).

3.5. The next fact is an immediate corollary of 3.4 and 3.2 (a), using the $W$ action and 1.61 .7

Lemma. Let $W^{\prime}$ be a facial subgroup of $W$. Then

(a) $\mathscr{K}_{W^{\prime}}=\mathscr{K} \cap \mathbb{R} \Pi_{W^{\prime}}=\mathscr{K} \cap \mathbb{R}_{\geq 0} \Pi_{W^{\prime}}$.

(b) $\mathscr{Z}_{W^{\prime}}=\mathscr{Z} \cap \mathbb{R} \Pi_{W^{\prime}}=\mathscr{Z} \cap \mathbb{R}_{\geq 0} \Pi_{W^{\prime}}$.

Remarks. (1) It is easy to see that, as $W$-subsets of $\mathbb{R} \Pi$, the cones $\mathscr{Y}, \mathscr{K}$ and $\mathscr{Z}$ are unchanged by extension or restriction of quadratic space.

(2) If $\Pi$ is linearly independent, the lemma remains true with "facial" replaced by "parabolic," by taking an ample extension and using (1) and Remark 2.3. Similar remarks apply to many other facts about the imaginary cone. 
3.6. We conclude this section with the following observations about the relation of the general case to the case of linearly independent simple roots. Consider $V^{\prime}, \Pi^{\prime}, \Phi^{\prime}, W^{\prime}, S^{\prime}, \mathscr{Q}^{\prime}$ and the linear map $L: V^{\prime} \rightarrow V$ associated to $V, \Pi, \Phi, W, S, \mathscr{Q}$ as in 1.4 Attach to $W^{\prime}$ acting on $V^{\prime}$ the fundamental chamber $\mathscr{C}^{\prime}$, Tits cone $\mathscr{X}^{\prime}$, imaginary cone $\mathscr{Z}^{\prime}$ and its fundamental domain $\mathscr{K}^{\prime}$ just as for $W$ acting on $V$.

Proposition. (a) $\mathbb{R}_{\geq 0} \Pi^{\prime} \cap L^{-1}(\{0\})=\{0\}$.

(b) $\mathscr{Q}^{\prime}=L^{-1}(\mathscr{Q}), \mathscr{C}^{\prime}=L^{-1}(\mathscr{C}), \mathscr{X}^{\prime}=L^{-1}(\mathscr{X}), \mathscr{K}^{\prime}=\mathbb{R}_{\geq 0} \Pi^{\prime} \cap L^{-1}(\mathscr{K})$ and $\mathscr{Z}^{\prime}=\mathbb{R}_{\geq 0} \Pi^{\prime} \cap L^{-1}(\mathscr{Z})$.

(c) L restricts to surjective maps $\mathbb{R}_{\geq 0} \Pi^{\prime} \rightarrow \mathbb{R}_{\geq 0} \Pi$, $\mathscr{K}^{\prime} \rightarrow \mathscr{K}$ and $\mathscr{Z}^{\prime} \rightarrow \mathscr{Z}$.

(d) L restricts to maps $\mathscr{Q}^{\prime} \rightarrow \mathscr{Q}, \mathscr{C}^{\prime} \rightarrow \mathscr{C}$ and $\mathscr{X}^{\prime} \rightarrow \mathscr{X}$; these maps are surjective if $L$ is surjective.

Remarks. None of the maps in (c)-(d) are bijective in general, since their domains and codomains are cones of possibly unequal dimension. There is also a map $\mathscr{Y}^{\prime} \rightarrow$ $\mathscr{Y}$ induced by $L$, but it is not obvious whether it satisfies analogues of the above properties.

Proof. Part (a) is equivalent to the assumed positive independence of $\Pi$. Part (b) follows from the definitions using the properties of $L$ stated in 1.4 and (c)-(d) then follow using (b) and the fact $L$ induces a bijection $\Pi^{\prime} \rightarrow \Pi$.

\section{Finiteness and NON-DEGEnERACy CONDitions}

4.1. Throughout Sections 4 11, the following conditions (i)-(iii) on $(V,\langle-,-\rangle)$ and $(\Phi, \Pi)$ are assumed except where explicitly indicated:

Assumption. (i) $V$ is finite dimensional

(ii) $\langle-,-\rangle$ is non-singular

(iii) $\Pi$ is finite.

We give $V$ its standard topology as finite dimensional real vector space, and we always consider subsets of $V$ in the induced topology unless otherwise indicated. We shall say that a subset $\Delta$ of $\Phi$, (usually of the form $\Delta=\Pi_{W^{\prime}}$ for some reflection subgroup $\left.W^{\prime}\right)$ is non-degenerate if the restriction of $\langle-,-\rangle$ to $\mathbb{R} \Delta$ is a non-singular form.

Remarks. The remainder of this paper makes more extensive use of standard properties of polyhedral cones (the most frequently used properties are listed in A.10. Some of the results and arguments below can be adapted to hold under more general hypotheses.

4.2. Simple properties of $\mathbb{R}_{\geq 0} \Pi$ and $\mathscr{C}$ are recorded below.

Lemma. (a) $\mathbb{R}_{\geq 0} \Pi$ and $\mathscr{C}=\left(\mathbb{R}_{\geq 0} \Pi\right)^{*}$ are dual polyhedral cones.

(b) The extreme rays of $\mathbb{R}_{\geq 0} \Pi$ are the sets $\mathbb{R}_{\geq 0} \alpha$ for $\alpha \in \Pi$.

(c) $\mathbb{R}_{\geq 0} \Pi$ is salient and $\mathscr{C}$ has an interior point $\rho$.

(d) We have $\langle\rho, \alpha\rangle>0$ for all $\alpha \in \Pi$. The intersection of $\mathbb{R}_{\geq 0} \Pi$ with the affine hyperplane $\{v \in V \mid\langle v, \rho\rangle=1\}$ is a convex polytope $P$ with vertices $\langle\rho, \alpha\rangle^{-1} \alpha$ for $\alpha \in \Pi$. Further, $P$ is a compact, convex base of $\mathbb{R}_{\geq 0} \Pi$.

(e) A subset $\Pi^{\prime}$ of $\Pi$ is facial if and only if it is a set of representatives of the extreme rays of some face of $\mathbb{R}_{\geq 0} \Pi$. 
(f) If $I$ is a facial subset of $S$ and $J \subseteq I$, then $J$ is a facial subset of $I$ (for the group $W_{I}$ with root system $\Phi_{I}$ and simple roots $\left.\Pi_{I}\right)$ ) if and only if $J$ is a facial subset of $S$.

(g) If $\Pi$ is linearly independent, then any subset of $\Pi$ is facial.

(h) If $\Pi=\Pi_{1} \dot{\cup} \Pi_{2}$ where $\mathbb{R} \Pi=\mathbb{R} \Pi_{1} \oplus \mathbb{R} \Pi_{2}$ (direct sum) then a subset $\Delta$ of $\Pi$ is facial if and only if $\Delta \cap \Pi_{i}$ is facial for $i=1,2$. This applies in particular if $\Pi_{1}$ is the union of some of the non-degenerate components of $\Pi$.

Proof. Part (a) follows using the definitions from 1.3(ii) and finiteness of $\Pi$. Part (b) holds since for $\alpha \in \Pi,\langle\alpha, \alpha\rangle>0$ while $\langle\alpha, \beta\rangle \leq 0$ for $\beta \in \Pi \backslash\{\alpha\}$ (i.e. the hyperplane $\alpha^{\perp}$ weakly separates $\alpha$ from $\Pi \backslash\{\alpha\}$ ). The first part of (c) follows from 1.3 (ii) and the second is well known to follow from the first (see A.11). Part (d) follows from (b)-(c) (see A.11 more generally). Part (e) follows from (a), the definition of facial subset of $\Pi$ and the fact $A .10(d)$ ), often taken as their definition, that faces of polyhedral cones are exposed faces. Part (f) also follows from Lemma A.10(d). Finally under the assumptions of $(\mathrm{g}), \mathbb{R}_{\geq 0} \Pi$ is a simplicial cone and so, using (e), any subset of $\Pi$ is facial as required. The straightforward proof of (h) is omitted.

4.3. The following lemma collects from the literature various consequences of the above assumptions 4.1(i)-(iii).

Lemma. (a) $W$ is discrete and closed in $\mathrm{GL}(V)$.

(b) $\Phi$ is discrete and closed in $V$.

(c) The interior $\operatorname{int}(\mathscr{X})$ of $\mathscr{X}$ consists of the set of all points $x$ of $\mathscr{X}$ which have finite stabilizer in $W$ i.e. such that $x^{\perp} \cap \Phi$ is finite.

(d) For any $x, y \in \operatorname{int}(\mathscr{X})$ there are only finitely many $\alpha \in \Phi_{+}$such that $\alpha^{\perp}$ contains a point of the closed interval with endpoints $x$ and $y$.

(e) For any $N \in \mathbb{R}_{>0}$ and $\rho \in \operatorname{int}(\mathscr{X})$, there are only finitely many $\alpha \in \Phi_{+}$ with $\langle\alpha, \rho\rangle \leq N$.

(f) There are only finitely many $W$-orbits of pairs $(\alpha, \beta)$ of roots such that $|\langle\alpha, \beta\rangle|<1$. In fact, each such pair is in the $W$-orbit of such a pair lying in the root system of finite standard parabolic subgroup of rank two.

(g) For any $N \in \mathbb{R}_{>1}$, there are only finitely many $W$-orbits of pairs $(\alpha, \beta)$ of roots with $1<|\langle\alpha, \beta\rangle| \leq N$.

Remarks. If $\Phi$ contains a root system of affine type as a parabolic subsystem, then there are infinitely many $W$-orbits of pairs $(\alpha, \beta)$ of roots with $\langle\alpha, \beta\rangle=1$.

Proof. We use 34 as a convenient source for proofs of most of these facts, though (excepting (e) and (g)) most parts can be found in or easily deduced from [49] or 4]. For (a), note that a discrete subgroup of a Hausdorff topological group is closed and see 44, Ch V, §4, Cor 3]. For (b), argue using (a) as in the proof of [34, Lemma 1.2.5]. For (c), see [34, Corollary 2.2.5]. Part (d) follows from (c) and Proposition 2.3(g). For (e), see [34, Lemma 5.7.1]. Part (f) follows using 4, Ch $\mathrm{V}, \S 4$, Ex 2(d)] (or [34, Proposition 3.1(a)]) and Lemma 2.9 since $\left\langle s_{\alpha}, s_{\beta}\right\rangle$ is finite when $-1<\langle\alpha, \beta\rangle<1$. For (g), see [34, Proposition 6.6.2].

4.4. It will be convenient to fix an element $\rho$ in the interior of $\mathscr{C}$ throughout the remainder of Sections 411 . Observe that the conditions (i)-(iii) on $(V,\langle-,-\rangle)$ and $(\Phi, \Pi)$ also are satisfied by $(V,\langle-,-\rangle)$ and $\left(\Phi_{W^{\prime}}, \Pi_{W^{\prime}}\right)$ for any finitely generated reflection subgroup $W^{\prime}$ of $W$. Hence all consequences of these conditions for $W$, 
including those in 4.3 and those given in the next lemma, apply to such reflection subgroups $W^{\prime}$ as well as to $W$.

Lemma. (a) If $v \in \mathscr{C}_{W}$, then $\{v-w v \mid w \in W\}$ is closed and discrete in $\mathbb{R}_{\geq 0} \Pi$, or equivalently, the orbit $W v$ of $v$ is closed and discrete in $V$.

(b) $\overline{\mathscr{X}}_{W}=\left\{v \in V \mid v+t \rho \in \mathscr{X}_{W}\right.$ for all $\left.t \in \mathbb{R}_{>0}\right\}$.

(c) $W$ has at most $\operatorname{dim}(\mathbb{R} \Pi)$ irreducible components.

(d) If $W_{1}, \ldots, W_{n}$ are reflection subgroups of $W$ (e.g. the components of $W$ ) which satisfy $\Phi=\cup_{i} \Phi_{W_{i}}$, then $\overline{\mathscr{X}_{W}}=\cap_{i=1}^{n} \overline{\mathscr{X}_{W_{i}}}$.

Proof. For (a), note that the closed polyhedral cone $\mathbb{R}_{\geq 0} \Pi$ is, by 4.2 (d), the union of compact sets (in fact, pyramidal polytopes) $\left\{v \in \mathbb{R}_{\geq 0} \Pi \mid\langle v, \rho\rangle \leq N\right\}$ for $N \in \mathbb{R}_{>0}$. Clearly, every point in $\mathbb{R}_{\geq 0} \Pi$ has a neighborhood (in $\mathbb{R}_{\geq 0} \Pi$ ) contained in one of these sets. To prove (a), it will therefore suffice, by 1.13 , to show that for fixed $v \in \mathscr{C}$ and for all $N \in \mathbb{R}_{>0}$,

$$
\{v-w v \mid w \in W,\langle v-w v, \rho\rangle \leq N\} \text { is finite. }
$$

By 1.13 for fixed $w \in W, v-w v=\sum_{i=1}^{n}\left\langle v, \alpha_{i}^{\vee}\right\rangle \beta_{i}$ where $\beta_{1}, \ldots, \beta_{n} \in \Phi_{+}$are distinct, $\alpha_{i} \in \Pi$ and $\left\langle v, \alpha_{i}\right\rangle \geq 0$. Let $A:=\left\{\left\langle v, \alpha^{\vee}\right\rangle \mid \alpha \in \Pi\right\} \cup\{0\} \subseteq \mathbb{R}_{\geq 0}$. If $A=\{0\}$, then $v=w v$ for all $w \in W$ and we are done. Otherwise, let $\epsilon:=$ $\min (A \backslash\{0\})>0$. Assume that $\langle\rho, v-w v\rangle \leq N$. Let $\Psi$ be the finite (by 4.3(e)) set of positive roots $\beta$ with $\langle\rho, \beta\rangle \leq N / \epsilon$. Then for any $i$ with $\left\langle v, \alpha_{i}\right\rangle \neq 0$, we have $\left\langle\rho, \beta_{i}\right\rangle \leq N / \epsilon$ i.e $\beta_{i} \in \Psi$. It follows that $v-w v=\sum_{\beta \in \Psi} c_{\beta} \beta$ for some $c_{\beta} \in A$. Since $\Psi$ and $A$ are finite, independent of $w$, there are only finitely many sums of this form, and (a) is proved.

Since $\mathscr{C} \subseteq \mathscr{X}$, we have that $\rho$ is an interior point of $\mathscr{X}$ and (b) follows (see Lemma A.3 more generally).

Let $W_{1}, \ldots, W_{n}$ be distinct irreducible components of $W$. Choose $\alpha_{i} \in \Pi_{W_{i}}$. Then $\left\langle\alpha_{i}, \alpha_{j}\right\rangle=\delta_{i, j}$ so $\alpha_{1}, \ldots, \alpha_{n}$ are distinct and linearly independent in $\mathbb{R} \Pi$. Hence $n$ is at most $\operatorname{dim}(\mathbb{R} \Pi)$, proving (c).

Part (d) follows from (b) and Lemma 1.10 (e)-(f), noting that $\rho$ is also an interior point of $\mathscr{C}_{W_{i}} \supseteq \mathscr{C}$ for all $i$.

4.5. Proofs of some results in the following sections proceed by reducing to the case of irreducible $W$ and considering cases depending on the type (finite, affine or indefinite) of $(W, S)$. The necessary background recalled below is from [49], though we use the terminology of [33] and express the results in terms of the cones $\mathbb{R}_{\geq 0} \Pi$, $\mathscr{K}$ and $\mathscr{X}$.

Assume that $W$ is finitely generated and irreducible. Let $A$ denote the $\Pi \times \Pi$ real matrix with entries $A_{\alpha, \beta}:=\langle\alpha, \beta\rangle$ for $\alpha, \beta \in \Pi$. Then the matrix $A$ is finite, indecomposable, symmetric and has non-positive off-diagonal entries. In particular, it satisfies the condition [33, (m1)-(m3)]. According to the classification there, $A$ is of finite, affine or indefinite type, and these types are mutually exclusive. In fact, $A$ is of finite type if and only if it is positive definite, and is of affine type if and only if it is positive semi-definite of corank 1. Otherwise, it is of indefinite type. Define the type of $\Phi$ (or $\Pi$ ) to be the same as that of $A$ (i.e. finite, affine or irreducible).

The matrix $A$ is of finite type if there is $v \in \mathbb{R}_{\geq 0} \Pi$ of the form $v=\sum_{\alpha \in \Pi} c_{\alpha} \alpha$ with all $c_{\alpha}>0$ such that $\langle v, \Pi\rangle \subseteq \mathbb{R}_{\geq 0}$. Then $(W, S)$ is an irreducible finite Coxeter system, $\Pi$ is linearly independent and if $v=\sum_{\alpha \in \Pi} c_{\alpha} \alpha$ with all $c_{\alpha} \in \mathbb{R}$ satisfies $\langle v, \Pi\rangle \subseteq \mathbb{R}_{\geq 0}$, then either $v=0$ or all $c_{\alpha}>0$. In particular, there is no non-zero 
$v \in \mathbb{R}_{\geq 0} \Pi$ such that $\langle v, \Pi\rangle \subseteq \mathbb{R}_{\leq 0}$. It follows from Lemma 1.10 (h) that $\mathscr{X}=V$, and from the properties above that $\mathscr{K}=0$, hence $\mathscr{Z}=0$ also.

The matrix $A$ is of affine type if there is $\delta \in \mathbb{R}_{\geq 0} \Pi$ of the form $\delta=\sum_{\alpha \in \Pi} c_{\alpha} \alpha$ with all $c_{\alpha}>0$ such that $\langle\delta, \Pi\rangle=\{0\}$. The group $W$ is an irreducible affine Weyl group. Moreover, up to rescaling the roots (multiplying them each by a positive scalar depending only on the $W$-orbit of the root), the root system $\Phi$ is the usual affine root system (of real roots) attached to the (crystallographic) root system of the corresponding finite Weyl group as in $[33$. The set $\Pi$ is linearly independent and the form $\langle-,-\rangle$ restricted to $\mathbb{R} \Pi$ is positive semi-definite with radical spanned by $\delta$. In particular, $\delta$ is uniquely determined up to multiplication by a positive scalar. If $v \in \mathbb{R} \Pi$ with $\langle v, \Pi\rangle \subseteq \mathbb{R}_{\geq 0}$ then $v \in \mathbb{R} \delta$ and so $\langle v, \Pi\rangle=0$. From the description of the untwisted affine root systems in [33, one has

$$
\mathscr{X}=\{v \in V \mid\langle v, \delta\rangle>0\} \cup\{v \in V \mid\langle v, \Pi\rangle=0\} .
$$

Note here that $\langle v, \Pi\rangle=0$ implies $\langle v, \delta\rangle=0$ so $\mathscr{X} \subseteq\{v \in V \mid\langle v, \delta\rangle \geq 0\}$. Moreover, $\{v \in \mathbb{R} \Pi \mid\langle v, \Pi\rangle=0\}=\mathbb{R} \delta$. These properties of affine type matrices imply that $\mathscr{K}=-\mathscr{C} \cap \mathbb{R}_{\geq 0} \Pi=\mathbb{R}_{\geq 0} \delta$ and hence that $\mathscr{Z}=W \mathscr{K}=\mathbb{R}_{\geq 0} \delta$ also. Call $\mathbb{R}_{\geq 0} \delta$ the isotropic ray of $\Pi$ (or of $\Phi$ or of $W$ ).

Now we describe the situation in case $A$ is of indefinite type. There is then some $\beta \in V$ such that $\beta=\sum_{\alpha \in \Pi} c_{\alpha} \alpha$ with all $c_{\alpha}>0$, and all $\langle\beta, \alpha\rangle<0$. Any such element $\beta$ is in the relative interior $\mathscr{K}^{0}$ of $\mathscr{K}$, and so $\mathbb{R} \mathscr{K}^{0}=\mathbb{R} \Pi$. If $v=\sum_{\alpha} d_{\alpha} \alpha \in \mathbb{R}_{\geq 0} \Pi$ where all $d_{\alpha} \geq 0$ and $\langle v, \Pi\rangle \subseteq \mathbb{R}_{\geq 0}$ then all $d_{\alpha}=0$. We do not have any more explicit description of $\mathscr{X}$ or $\mathscr{Z}$ than that given by the general results and definitions already given, though we shall give several other descriptions of $\overline{\mathscr{Z}}$ and one of $\mathscr{K}$ in the next section.

Define the type of $\Pi, \Phi$, or $W$ to be that of the matrix $A$ above (so the type is either finite, affine or indefinite). We shall say that $A$ (or $\Pi$, or $\Phi$, or $W$ ) is of infinite type if it is of affine type or indefinite type.

Remarks. (1) If $(W, S)$ is infinite dihedral, then $A$ above may be of either affine or indefinite type, depending on the root system $\Phi$. In any other (finite rank irreducible) case, the type of $A$ coincides with the type (finite, affine or indefinite) of $(W, S)$ in the usual sense.

(2) Assume that $(W, S)$ is infinite irreducible of finite rank at least three. Then $(W, S)$ is of affine type if and only if it has a free abelian subgroup of finite index in $W$, or equivalently, if " $W$ is of polynomial growth" (that is, there exist $C \in \mathbb{R}_{>0}$, $k \in \mathbb{N}$ such that $|\{w \in W \mid l(w) \leq n\}| \leq C\left(n^{k}+1\right)$ for all $\left.n \in \mathbb{N}\right)$. Further, $(W, S)$ is of indefinite type if and only if it has a non-abelian free group as subgroup, or equivalently, if " $W$ is of exponential growth" (that is, there exist $\lambda \in \mathbb{R}_{>1}$ such that $|\{w \in W \mid l(w) \leq n\}| \geq \lambda^{n}$ for all $\left.n \in \mathbb{N}\right)$. See [11] and 36].

(3) If $A$ is of infinite type, then $\mathscr{X} \cap-\mathscr{X}=\mathscr{C} \cap-\mathscr{C}=\{v \in V \mid\langle v, \Pi\rangle=0\}$ by the argument of the proof of [31, Proposition 3.2] (see also [34, 49]).

\section{The Closed imaginary CONE}

In this section, we give several characterizations of the closed imaginary cone. The analogous results for root systems and Weyl groups of Kac-Moody Lie algebras were proved by Kac (see [33, Ch 5, especially Section 5.14]). 
5.1. We first show that (under the standing assumptions 4.1(i)-(iii)), the closure of $\mathscr{Z}$ is the dual of the Tits cone (cf. [33, $\S 5.8]$ ).

Theorem. (a) The closures $\overline{\mathscr{X}}$ and $\overline{\mathscr{Z}}$ of $\mathscr{X}$ and $\mathscr{Z}$ are dual cones.

(b) $\overline{\mathscr{Z}}=\mathscr{Y}$.

(c) If $W^{\prime}$ is a finitely generated facial subgroup of $W$, then $\overline{\mathscr{Z}_{W^{\prime}}}=\overline{\mathscr{Z}} \cap \mathbb{R} \Pi_{W^{\prime}}$.

(d) $\overline{\mathscr{Z}}=\sum_{i=1}^{n} \overline{\mathscr{Z}_{W_{i}}}$ if $W_{1}, \ldots, W_{n}$ are finite rank reflection subgroups (e.g. the components of $W$ ) such that $\Phi=\cup_{i=1}^{n} \Phi_{W_{i}}$.

Proof. First we prove (a). We have $\mathscr{Z} \subseteq \mathbb{R}_{\geq 0} \Pi$, so taking duals using Lemma 3.1(d) shows that $\mathscr{C} \subseteq \mathscr{Z}^{*}$. Since $\mathscr{Z}$ is $W$-invariant, so is $\mathscr{Z}^{*}$ and hence $\mathscr{X}=$ $\cup_{w \in W} w \mathscr{C} \subseteq \mathscr{Z}^{*}$. Since $\mathscr{Z}^{*}=\overline{\mathscr{Z}}^{*}$ is closed, we get that $\overline{\mathscr{X}} \subseteq \overline{\mathscr{Z}}^{*}$.

We shall prove the reverse inclusion first under the additional assumption that $(W, S)$ is irreducible. If $\Phi$ is of finite type, then $\overline{\mathscr{X}}=V$ and $\overline{\mathscr{Z}}=0$ so the result holds. If $\Phi$ is of affine type, then $\overline{\mathscr{X}}=\{v \in V \mid\langle v, \delta\rangle \geq 0\}$ and $\overline{\mathscr{Z}}=\mathbb{R}_{\geq 0} \delta$, so the result holds in this case also.

Now assume that $\Phi$ is of indefinite type. Choose $\beta \in \mathscr{K}$ and $\epsilon>0$ such that $\beta=\sum_{\alpha \in \Pi} d_{\alpha} \alpha$ with $d_{\alpha}>0$ and $\left\langle\beta, \alpha^{\vee}\right\rangle<-\epsilon$ for all $\alpha \in \Pi$. Consider $z \in \mathscr{Z}^{*}$ and $\gamma \in \Phi_{+}$. Write (by [3, Lemma 3.3]) $\gamma^{\vee}=\sum_{\alpha \in \Pi} c_{\alpha} \alpha^{\vee}$ where all $c_{\alpha} \geq 0$ and some $c_{\alpha} \geq 1$. We have $s_{\gamma}(\beta)=\beta+s \gamma$ where

$$
s=-\left\langle\beta, \gamma^{\vee}\right\rangle=\sum_{\alpha} c_{\alpha}\left(-\left\langle\beta, \alpha^{\vee}\right\rangle\right)>\epsilon .
$$

But $\beta$ and $s_{\gamma}(\beta)$ are in $\mathscr{Z}$, so by definition of $\mathscr{Z}^{*}$, we have $\langle z, \beta\rangle \geq 0$ and $\left\langle z, s_{\gamma}(\beta)\right\rangle \geq 0$. Here, $\left\langle z, s_{\gamma} \beta\right\rangle=\langle z, \beta\rangle+s\langle z, \gamma\rangle$ so $\langle z, \gamma\rangle \geq-\frac{1}{s}\langle z, \beta\rangle \geq$ $-\epsilon^{-1}\langle z, \beta\rangle$. Hence for $t \in \mathbb{R}_{>0}$,

$$
\langle z+t \rho, \gamma\rangle \geq-\frac{1}{\epsilon}\langle z, \beta\rangle+t\langle\rho, \gamma\rangle \text {. }
$$

This implies that if $\langle z+t \rho, \gamma\rangle\left\langle 0\right.$, then $\langle\rho, \gamma\rangle \leq \frac{1}{\epsilon t}\langle z, \gamma\rangle$. For fixed $t>0$, there are only finitely many $\gamma \in \Phi_{+}$satisfying this latter condition by Lemma $4.3(\mathrm{e})$, and it follows by Lemma 1.10 (a) that $z+t \rho \in \mathscr{X}$. Since $t \in \mathbb{R}_{>0}$ was arbitrary, we get $z \in \overline{\mathscr{X}}$ by Lemma 4 .4 b). This completes the proof that $\overline{\mathscr{Z}}^{*}=\overline{\mathscr{X}}$ if $(W, S)$ is irreducible.

To prove $\overline{\mathscr{Z}}^{*}=\overline{\mathscr{X}}$ in general, let $W_{1}, \ldots, W_{n}$ be the irreducible components of $W$. Then from above and the Lemmas 3.2 (d) and 4.4(d), we have

$$
\overline{\mathscr{Z}}^{*}=\mathscr{Z}^{*}=\left(\sum_{i} \mathscr{Z}_{W_{i}}\right)^{*}=\bigcap_{i} \mathscr{Z}_{W_{i}}^{*}=\bigcap_{i}{\overline{\mathscr{Z}} W_{i}}^{*}=\bigcap_{i} \overline{\mathscr{X}_{W_{i}}}=\overline{\mathscr{X}} .
$$

Part (b), (c) are restatements using (a) of Lemma 3.1(d),(c) while (d) follows by taking dual cones in Lemma 4.3 (a) using (a). Several other results from $\$ 34$ admit similar restatements using (a) which we shall not list explicitly.

5.2. Subsections 5.355 give another description of the closure of $\mathscr{Z}$ (cf. [33, Lemma 5.8 and Exercise 5.12]), using a topology on the set of rays in $V$ which is defined in this subsection.

Let $\widetilde{R}:=\left\{\mathbb{R}_{\geq 0} \alpha \mid \alpha \in V \backslash\{0\}\right\}$ denote the set of rays of $V$ (see 1.1). For any $E \subseteq \widetilde{R}$, let $\cup E:=\cup_{e \in E} e \subseteq V$ denote the union of the rays $e$ in the set $E$. If $V=\{0\}$, then $\widetilde{R}=\emptyset$, which we give its only possible topology. To avoid trivialities, assume henceforward that $V \neq\{0\}$. Choose a compact convex body $B$ 
(e.g. a closed ball) with 0 in its interior, and let $B^{\prime}$ denote the boundary of $B$. The map $\widetilde{R} \rightarrow B^{\prime}$ taking each ray to the unique point of $B^{\prime}$ which the ray contains is a bijection, and we topologize $\widetilde{R}$ by declaring this map to be a homeomorphism. This gives $\widetilde{R}$ a topology, independent of the choice of $B$, in which it is homeomorphic to the standard $(\operatorname{dim}(V)-1)$-sphere. Note that $W$ acts naturally on $\widetilde{R}$ as a group of homeomorphisms; this action is faithful since if an element of $w$ fixes all rays, it cannot make any positive root negative and so must be the identity. One may find in A.11 A.12 some useful well known facts concerning the subset of $R$ which has as its elements the rays contained in any fixed closed salient cone.

For any pointed, possibly non-convex cone $X \subseteq V$ let $\operatorname{ray}(X):=\left\{\mathbb{R}_{\geq 0} \alpha \mid \alpha \in\right.$ $X \backslash\{0\}\} \subseteq \widetilde{R}$ denote the set of rays of $V$ through non-zero points of $X$, topologized as a subspace of $\widetilde{R}$. We call it the space of rays of $X$. In particular, $\widetilde{R}=\operatorname{ray}(V)$. For $R \subseteq \widetilde{R}$, let $\cup R \subseteq V$ be the possibly non-convex pointed cone arising as the union of all rays in $R$. The maps $X \mapsto R:=\operatorname{ray}(X)$ and $R \mapsto X:=\cup R$ define inverse bijections between the set of pointed, possibly non-convex cones in $V$ and the power set of $\operatorname{ray}(V)$.

5.3. Let $Q:=\operatorname{ray}(\mathscr{Q}) \subseteq \operatorname{ray}(V)$ denote the set all isotropic rays of $V, R_{+}:=$ $\left\{\mathbb{R}_{\geq 0} \alpha \mid \alpha \in \Phi_{+}\right\} \subseteq \operatorname{ray}(V)$ and $R_{0}:=\overline{R_{+}} \backslash R_{+}$. Results closely related to the following appear in [30.

Proposition. (a) $R_{+}$consists of positive rays and is discrete in ray $(V)$.

(b) $R_{0} \subseteq Q$ and $R_{0}$ is closed in $\operatorname{ray}(V)$.

(c) $R_{0}$ is the set of limit rays (i.e. limit points) of $R_{+}$i.e. $R_{0}=\operatorname{Acc}\left(R_{+}\right)$.

Proof. Consider the set $R_{\Pi}:=\operatorname{ray}\left(\mathbb{R}_{\geq 0} \Pi\right) \subseteq \operatorname{ray}(V)$ of rays of $V$ contained in $\mathbb{R}_{\geq 0} \Pi$. Since $\langle\rho, \alpha\rangle>0$ for all $\alpha \in \Pi$, we may define a map

$$
\tau: \mathbb{R}_{\geq 0} \Pi \backslash\{0\} \rightarrow H:=\{v \in V \mid\langle v, \rho\rangle=1\}
$$

by $v \mapsto\langle v, \rho\rangle^{-1} v$. The image of $\tau$ is the set $P:=\left\{v \in \mathbb{R}_{\geq 0} \Pi \mid\langle\rho, v\rangle=1\right\}$, which by Lemma 4.2 (d), is a convex polytope in the affine hyperplane $H$, with the points $\langle\rho, \alpha\rangle^{-1} \alpha$ for $\alpha \in \Pi$ as vertices. We may and do choose $B$ above so that $P \subseteq B^{\prime}$. It follows that the map taking a ray in $R_{\Pi}$ to its intersection with $P$ is a homeomorphism $\theta: R_{\Pi} \stackrel{\cong}{\rightarrow} P$, explicitly given by $\mathbb{R}_{\geq 0} \alpha \mapsto \tau(\alpha)$ for non-zero $\alpha \in \mathbb{R}_{\geq 0} \Pi$. (This is essentially the realization of rays in $\mathbb{R}_{\geq 0} \Pi$ used in [30, the possible hyperplanes $V_{1}$ "transverse" to $\Pi$ used there are exactly the hyperplanes $H$ as above for varying $\rho$ ). Clearly, $R_{\Pi}$ is closed (in fact, compact) and $R_{+} \subseteq R_{\Pi}$, so $R_{0} \subseteq \bar{R}_{+} \subseteq R_{\Pi}$ also.

Now $\left.\theta\left(\overline{R_{+}}\right)=\overline{\tau\left(\Phi_{+}\right.}\right)$. The right hand side consists of all limit points of sequences $\left(\tau\left(\alpha_{n}\right)\right)$ (in $P$ ) for sequences $\left(\alpha_{n}\right)_{n \in \mathbb{N}}$ of positive roots. Consider a limit point $\alpha \in P$ of such a sequence. We may assume without loss of generality that the sequence $\left(\tau\left(\alpha_{n}\right)\right)$ actually converges to $\alpha$. We consider two possibilities. The first case is that the sequence $\left(p_{n}\right):=\left(\left\langle\rho, \alpha_{n}\right\rangle\right)$ is bounded. Then by Lemma 4.3(e), there are only finitely many possibilities for each $\alpha_{n}$. In this case, the sequences $\left(\alpha_{n}\right)$ and $\left(\tau\left(\alpha_{n}\right)\right)$ must be eventually constant, and $\alpha=\tau\left(\alpha_{n}\right)$ for all sufficiently large $n$. This corresponds to an (obviously positive) limit ray $\theta^{-1}(\alpha)$ in $R_{+}$. In the contrary case, the sequence $p_{n}$ is unbounded, and passing to a subsequence we may assume it has limit $+\infty$. We have

$$
\langle\alpha, \alpha\rangle=\lim _{n \rightarrow \infty}\left\langle\tau\left(\alpha_{n}\right), \tau\left(\alpha_{n}\right)\right\rangle=\lim _{n \rightarrow \infty} p_{n}^{-2}\left\langle\alpha_{n}, \alpha_{n}\right\rangle=\lim _{n \rightarrow \infty} p_{n}^{-2}=0 .
$$


This case yields an isotropic limit ray $\theta^{-1}(\alpha) \in R_{0}$. Observe that any limit of a sequence of rays in $R_{0}$ is obviously an isotropic ray and is contained in $\overline{R_{+}}$, so it must be in $R_{0}$. Therefore $R_{0}$ is closed. From the above, $R_{+}$is discrete, as any sequence in $R_{+}$which converges to an element of $R_{+}$is eventually constant (since otherwise it converges to an isotropic ray). This completes the proof of (a)-(c).

5.4. Now we show the closed imaginary cone is the conical closure of the union of the limit rays of the set of rays spanned by positive roots.

Theorem. The imaginary cone $\mathscr{Z}$ satisfies $\overline{\mathscr{Z}}=\mathbb{R}_{\geq 0}\left(\bigcup R_{0}\right)$.

Proof. Maintain notation and assumptions from the proof of the preceding proposition. Since $R_{0}$ is topologically closed, $\theta\left(R_{0}\right)$ is a topologically closed, hence compact subset of the polytope $P$. The conical closure $F$ of $\bigcup_{r \in R_{0}} r \cup\{0\}$ is equal to that of $\theta\left(R_{0}\right) \cup\{0\}$. Let $K$ be the convex closure of $\theta\left(R_{0}\right)$ in $P$; it is compact since $\theta\left(R_{0}\right)$ is topologically closed. Clearly, $F=\left\{\lambda k \mid \lambda \in \mathbb{R}_{\geq 0}, k \in K\right\}$, which is easily seen to be closed in $V$.

To prove the theorem, we first reduce to the case that $W$ is irreducible. Let $W_{1}, \ldots, W_{n}$ be the irreducible components of $W$. Denote the analogues of $R, R_{+}$ and $R_{0}$ for $W_{i}$ as $R_{W_{i}}, R_{W_{i},+}$ and $R_{W_{i}, 0}$ respectively. Since $R_{+}=\cup_{i} R_{W_{i},+}$ (disjoint union) we have $\overline{R_{+}}=\cup_{i} \overline{R_{W_{i},+}}$. Note $R_{W_{i},+} \cap R_{W_{j}, 0}=\emptyset$ (since a ray cannot be both positive and isotropic). Therefore $R_{0}=\cup_{i} R_{W_{i}, 0}$. If the theorem is known for irreducible Coxeter groups, then the conical closure of $\cup_{r \in R_{W_{i}, 0}} r \cup\{0\}$ is $\overline{\mathscr{Z}_{W_{i}}}$ and hence the conical closure $F$ of $\cup_{r \in R_{0}} r \cup\{0\}$ is $\sum_{i} \overline{\mathscr{Z}_{W_{i}}}$. Now $F$ is topologically closed from above, so $F$ is the topological closure of $\sum_{i} \mathscr{Z}_{W_{i}}$, which in turn is the topological closure of $\mathscr{Z}$ as required by Lemma $3.2(\mathrm{~d})$.

We now may and do assume that $W$ is irreducible. If $\Phi$ is of finite type, then $R_{0}=\emptyset$ since $R_{+}$is finite, and $\overline{\mathscr{Z}}=0$ so the result holds in this case. If $\Phi$ is of affine type, then using the standard description of the root system of affine Weyl groups, one easily sees $R_{0}=\left\{\mathbb{R}_{\geq 0} \delta\right\}$ where $\delta$ is as in 4.5 , and $F=\mathbb{R}_{\geq 0} \delta=\overline{\mathscr{Z}}$ as required. Henceforward we assume that $\Phi$ is of indefinite type. Since $\bar{F}$ is a closed cone, it will suffice by Theorem 5.3 to show that $F \subseteq \overline{\mathscr{Z}}$ and $F^{*} \subseteq \overline{\mathscr{X}}$.

We shall first show that $\theta\left(R_{0}\right) \subseteq \overline{\mathscr{Z}}$, which implies that $F \subseteq \overline{\mathscr{Z}}$. Fix $\beta \in \mathscr{K}$ and $\epsilon>0$ with $\left\langle\beta, \alpha^{\vee}\right\rangle<-\epsilon$ for all $\alpha \in \Pi$. For any root $\gamma \in \Phi_{+}$, we may write $\gamma^{\vee}=$ $\sum_{\alpha \in \Pi} c_{\alpha} \alpha^{\vee}$ where all $c_{\alpha} \geq 0$ and some $c_{\alpha} \geq 1$. Then $s_{\gamma}(\beta)=\beta+s \gamma \in \mathscr{Z}$ where $s=-\left\langle\beta, \gamma^{\vee}\right\rangle \geq \epsilon$ and $\langle\rho, \beta\rangle>0$. Hence $\left\langle\rho, s_{\gamma}(\beta)\right\rangle=\langle\rho, \beta\rangle+s\langle\rho, \gamma\rangle \geq \epsilon\langle\rho, \gamma\rangle$. One computes that

$$
e_{\gamma}:=\tau(\gamma)-\tau\left(s_{\gamma}(\beta)\right)=a_{\gamma} \gamma-b_{\gamma} \beta
$$

where

Here,

$$
a_{\gamma}=\frac{\langle\rho, \beta\rangle}{\langle\rho, \gamma\rangle\left\langle\rho, s_{\gamma}(\beta)\right\rangle}, \quad b_{\gamma}=\frac{1}{\left\langle\rho, s_{\gamma}(\beta)\right\rangle}
$$

$$
0 \leq\left\langle\rho, a_{\gamma} \gamma\right\rangle \leq \frac{\langle\rho, \beta\rangle}{\epsilon\langle\rho, \gamma\rangle}, \quad 0 \leq\left\langle\rho, b_{\gamma} \beta\right\rangle \leq \frac{\langle\rho, \beta\rangle}{\epsilon\langle\rho, \gamma\rangle}
$$

Let $f \in \theta\left(R_{0}\right)$. Then there exists a sequence $\left(\gamma_{n}\right)_{n \in \mathbb{N}}$ of positive roots with $\lim _{n \rightarrow \infty}\left\langle\rho, \gamma_{n}\right\rangle=\infty$ and $\lim _{n \rightarrow \infty} \tau\left(\gamma_{n}\right)=f$. Note that $a_{\gamma_{n}} \gamma_{n}$ and $b_{\gamma_{n}} \beta$ are in $\mathbb{R}_{\geq 0} \Pi$ while

$$
\lim _{n \rightarrow \infty}\left\langle\rho, a_{\gamma_{n}} \gamma_{n}\right\rangle=0=\lim _{n \rightarrow \infty}\left\langle\rho, b_{\gamma_{n}} \beta\right\rangle
$$


It follows that

$$
\lim _{n \rightarrow \infty} a_{\gamma_{n}} \gamma_{n}=0=\lim _{n \rightarrow \infty} b_{\gamma_{n}} \beta
$$

and hence $\lim _{n \rightarrow \infty} e_{\gamma_{n}}=0$. Therefore

$$
\lim _{n \rightarrow \infty} \tau\left(s_{\gamma_{n}}(\beta)\right)=\lim _{n \rightarrow \infty} \tau\left(\gamma_{n}\right)=f .
$$

Since $\tau\left(s_{\gamma_{n}}(\beta)\right) \in \mathscr{Z}$, we deduce that $f \in \overline{\mathscr{Z}}$. This completes the proof that $\theta\left(R_{0}\right) \subseteq \overline{\mathscr{Z}}$

We have proved that $F \subseteq \overline{\mathscr{Z}}$. It remains to prove that $F^{*} \subseteq \overline{\mathscr{X}}$. For this, it will suffice by Lemma $4.4(\mathrm{~b})$ to show that if $x \in V$ with $\left\langle x, \theta\left(R_{0}\right)\right\rangle \subseteq \mathbb{R}_{\geq 0}$, then $x+t \rho \in \mathscr{X}$ for all $t \in \mathbb{R}_{>0}$. If $x+t \rho \notin \mathscr{X}$ for some $t>0$, there is by Lemma 1.10 (a) a sequence of distinct roots $\gamma_{n} \in \Phi_{+}$with $\left\langle x+t \rho, \gamma_{n}\right\rangle<0$ for all $n$. Passing to a subsequence, we may assume by Lemma 4.3 (e) that $\lim _{n \rightarrow \infty}\left\langle\rho, \gamma_{n}\right\rangle=\infty$ and $\lim _{n \rightarrow \infty} \tau\left(\gamma_{n}\right)=f \in \theta\left(R_{0}\right)$. We have $\langle\rho, f\rangle=1$, and by assumption on $x$, we have $\langle x, f\rangle \geq 0$, and so $\langle x+t \rho, f\rangle \geq t>0$. On the other hand, from $\left\langle x+t \rho, \gamma_{n}\right\rangle<0$ for all $n$, it follows that

$$
\langle x+t \rho, f\rangle=\lim _{n \rightarrow \infty}\left\langle x+t \rho, \tau\left(\gamma_{n}\right)\right\rangle \leq 0,
$$

a contradiction which completes the proof.

5.5. For each reflection subgroup $W^{\prime}$ of $\Phi$, let $R_{W^{\prime},+}:=\left\{\mathbb{R}_{\geq 0} \alpha \mid \alpha \in \Phi_{W^{\prime},+}\right\}$ and $R_{W^{\prime}, 0}:=\overline{R_{W^{\prime},+}} \backslash R_{W^{\prime},+}$. These are subsets of $\operatorname{ray}\left(\mathbb{R}_{\geq 0} \Pi^{\prime}\right) \subseteq \operatorname{ray}\left(\mathbb{R}_{\geq 0} \Pi\right) \subseteq \operatorname{ray}(V)$.

Corollary. Let $W^{\prime}$ be a reflection subgroup of $W$. Then

(a) $R_{W^{\prime},+} \subseteq R_{+}$

(b) $R_{W^{\prime}, 0} \subseteq R_{0}$.

(c) $R_{W^{\prime}, 0}=\emptyset$ if $W^{\prime}$ is finite.

(d) If $W^{\prime}$ is infinite dihedral, then $R_{W^{\prime}, 0}$ is a single $W^{\prime}$-orbit of rays and consists of a set of one (resp., two) isotropic rays of $V$ according as to whether $W^{\prime}$ is of affine or indefinite type.

(e) $\overline{\mathscr{Z}}_{W^{\prime}} \subseteq \overline{\mathscr{Z}}$.

Proof. Part (a) is obvious, and (b) holds since since $R_{W^{\prime}, 0} \subseteq \overline{R_{+}}$contains only isotropic rays. Part (c) holds since $R_{W^{\prime},+}$ is finite (and hence closed) if $W$ is finite, and (d) is well known (see for instance 30]). For the proof of (e), assume without loss of generality by Theorem $3.3(\mathrm{~b})$ that $W^{\prime}$ is finitely generated. Then (e) follows by taking closed convex hulls of the union of each side of (b) and using Theorem 5.3 (a) (or from Lemma 3.1 (a) and Theorem 5.1).

5.6. The set of limit rays of rays spanned by positive roots has been independently studied in [30, which contains, as well as some basic results not proved here, several instructive examples (including diagrams in low rank). For use later in this paper and in [21, we reformulate below in the framework of this paper a fundamental fact proved in 30, which implies in particular that the set of limit rays of positive roots is the closure of the union of the sets of limit rays of positive roots of dihedral reflection subgroups. Let $R_{0}^{\prime}$ (resp., $R_{0}^{\prime \prime}$ ) denote $\bigcup_{W^{\prime}} R_{W^{\prime}, 0}$ where the union is over the infinite dihedral reflection subgroups $W^{\prime}$ of $W$ (resp., the infinite dihedral reflection subgroups $W^{\prime}$ containing a simple reflection of $W$ i.e. with $\left.\Pi_{W^{\prime}} \cap \Pi \neq \emptyset\right)$.

Theorem. (a) $R_{0}^{\prime \prime} \subseteq R_{0}^{\prime} \subseteq R_{0}$.

(b) $\left(\left[30\right.\right.$, Theorems 4.2 and 4.5]) $\overline{R_{0}^{\prime \prime}}=\overline{R_{0}^{\prime}}=R_{0}$. 
(c) $\overline{\mathscr{Z}}=\mathbb{R}_{\geq 0}\left(\bigcup R_{0}\right)=\mathbb{R}_{\geq 0}\left(\operatorname{cl}\left(\bigcup R_{0}^{\prime}\right)\right)=\mathbb{R}_{\geq 0}\left(\operatorname{cl}\left(\bigcup R_{0}^{\prime \prime}\right)\right)$.

Remarks. The validity of the weaker result in (b), that $\overline{R_{0}^{\prime}}=R_{0}$, was raised as a question in an earlier version of this paper.

Proof. Part (a) is obvious from Corollary 5.5. For (b), choose the convex body $B$ in 5.3 so that $P:=\left\{v \in \mathbb{R}_{\geq_{0}} \Pi \mid\langle v, \rho\rangle=1\right\}$ is a subset of the boundary $B^{\prime}$ of $B$ and thereby identify $P$ as a compact subset of $\operatorname{ray}(V)$. For a reflection subgroup $W^{\prime}, \Psi_{W^{\prime},+}:=\left\{\rho(\alpha)^{-1} \alpha \mid \alpha \in \Phi_{W^{\prime},+}\right\} \subseteq P$ was called the set of normalized roots of $W^{\prime}$ in 30. Define $E\left(W^{\prime}\right):=\overline{\Psi_{W^{\prime},+}} \backslash \Psi_{W^{\prime},+}$, and $E=E(W)$. Also, let $E_{2}$ (resp., $E_{2}^{\circ}$ ) be the union of the sets $E\left(W^{\prime}\right)$ for $W^{\prime}$ ranging over the infinite dihedral reflection subgroups $W^{\prime}$ of $W$ (resp., the infinite dihedral reflection subgroups $W^{\prime}$ with $\left.\Pi_{W^{\prime}} \cap \Pi \neq \emptyset\right)$. The cited results from 30 then are the statements that $\overline{E_{2}^{\circ}}=\overline{E_{2}}=E$, which are obviously equivalent to (b) via the above identification. From Lemma A.12, it follows from (b) that $\operatorname{cl}\left(\bigcup_{r \in R_{0}^{\prime}} r\right)=\operatorname{cl}\left(\bigcup_{r \in R_{0}^{\prime \prime}} r\right)=R_{0}$, and then (c) follows by Theorem 5.4 .

5.7. We introduce next some notation for several families of facial subsets of $S$ which we shall consider subsequently. Let $F_{\text {all }}$ be the set of all facial subsets of $S$ and $F_{\mathrm{m}}$ be the set of all maximal proper facial subsets of $S$. Also, let $F_{\mathrm{m} \text {.ind }}$ (resp., $F_{\text {inf }}$ ) be the set of all elements $I$ of $F_{\mathrm{m}}$ (resp., of $F_{\text {all }}$ ) such that $\Pi_{I}$ has all its irreducible components of indefinite type (resp., infinite type). Thus, $F_{\text {inf }}$ consists of the special facial subsets of $S$.

5.8. We now give a description of $\mathscr{K}$ and $\overline{\mathscr{Z}}$ which is special to the case of irreducible $W$ of indefinite type (see [33, Exercise 5.11]).

Proposition. Suppose that $\Pi$ is irreducible of indefinite type. For each $I \in F_{\text {all }}$, choose $\phi_{I} \in \mathscr{C}$ such that $\Pi_{I}=\Pi \cap \phi_{I}^{\perp}$. Then

(a) $\mathscr{K}=\left\{v \in-\mathscr{C} \cap \mathbb{R} \Pi \mid\left\langle v, \phi_{I}\right\rangle \geq 0\right.$ for all $\left.I \in F_{\text {m.ind }}\right\}$.

(b) $\overline{\mathscr{Z}}=\left\{v \in-\overline{\mathscr{X}} \cap \mathbb{R} \Pi \mid\left\langle v, w \phi_{I}\right\rangle \geq 0\right.$ for all $I \in F_{\text {m.ind }}$ and $\left.w \in W\right\}$.

Proof. Recall the notation $H_{\phi}^{\prec}$ of 2.3 . We use below the fact that any polyhedral cone of maximum possible dimensional in a finite dimension in real vector space is the intersection of a unique minimal family of closed half-spaces (corresponding naturally to the facets (maximal proper faces) of the cone). For $\mathbb{R}_{>0} \Pi$ in $\mathbb{R} \Pi$, this gives $\mathbb{R}_{\geq 0} \Pi=\bigcap_{I \in F_{\mathrm{m}}}\left(\mathbb{R} \Pi \cap H_{\phi_{I}}^{>}\right)$. Since $-\mathscr{C}=\cap_{\phi \in-\Pi} H_{\phi}^{>}$, the polyhedral cone $\mathscr{K}$ is the intersection of the half-spaces $\mathbb{R} \Pi \cap H_{\bar{\phi}}^{\geq}$of $\mathbb{R} \Pi$ for $\phi \in \Gamma:=-\Pi \cup\left\{\phi_{I} \mid I \in F_{\mathrm{m}}\right\}$. Since $\mathscr{K}$ is a full-dimensional polyhedral cone in $\mathbb{R} \Pi$, in order to prove (a), it will suffice to show that for each $I \in F_{\mathrm{m}} \backslash F_{\mathrm{m} \text {.ind }}, \mathscr{K}$ is the intersection of the closed half-spaces $H_{\bar{\phi}}^{\gtrless} \cap \mathbb{R} \Pi$ of $\mathbb{R} \Pi$ for $\phi \in \Gamma_{I}:=\Gamma \backslash\left\{\phi_{I}\right\}$.

Suppose to the contrary that $z \in \cap_{\phi \in \Gamma_{I}}\left(\mathbb{R} \Pi \cap H_{\phi}^{>}\right) \backslash \mathscr{K}$. Then $\left\langle z, \phi_{I}\right\rangle<0$. Since $\Pi$ is of indefinite type, we may choose $\beta=\sum_{\alpha \in \Pi} c_{\alpha} \alpha$ with all $c_{\alpha} \in \mathbb{R}_{>0}$ and $\langle\beta, \alpha\rangle<0$ for all $\alpha \in \Pi$. In particular, $\left\langle z, \phi_{I}\right\rangle<0$ and $\left\langle\beta, \phi_{I}\right\rangle>0$, so there is a unique real number $t$ with $0<t<1$ such that $z^{\prime}:=t z+(1-t) \beta$ satisfies $\left\langle z^{\prime}, \phi_{I}\right\rangle=0$. For all $\alpha \in \Pi,\langle z, \alpha\rangle \leq 0$ and $\langle\beta, \alpha\rangle<0$ so $\left\langle z^{\prime}, \alpha\right\rangle<0$. Since $\left\langle z, \phi_{J}\right\rangle \geq 0$ and $\left\langle\beta, \phi_{J}\right\rangle>0$ for all $J \in \Gamma_{I}$, we have $\left\langle z^{\prime}, \phi_{J}\right\rangle>0$ for all such $J$, and $\left\langle z^{\prime}, \phi_{I}\right\rangle=0$ by choice of $t$. So $z^{\prime}$ lies in the facet $\mathbb{R}_{\geq 0} \Pi_{I}=\mathbb{R}_{\geq 0} \Pi \cap \phi_{I}^{\perp}$ of $\mathbb{R}_{\geq 0} \Pi$ but is in none of the other facets. Hence we may write $z^{\prime}=\sum_{\alpha \in \Pi_{I}} d_{\alpha} \alpha$ with all $d_{\alpha}>0$. The above also showed that $\left\langle z^{\prime}, \alpha\right\rangle<0$ for all $\alpha \in \Pi_{I}$. But by 4.5 , this 
implies that each component of $\Pi_{I}$ is of indefinite type and so $I \in F_{\mathrm{m} \text {.ind }}$, contrary to assumption. This proves (a).

For (b), first note that $\mathscr{Z}=\mathscr{Y} \cap-\mathscr{X}$ by Lemma $3.2($ a), so $\overline{\mathscr{Z}} \subseteq-\overline{\mathscr{X}}$. Also, $\mathscr{Z} \subseteq \mathbb{R} \Pi$ and

$$
\mathscr{Z} \subseteq \mathscr{Y}=\bigcap_{w \in W} w\left(\mathbb{R}_{\geq 0} \Pi\right)=\bigcap_{\substack{w \in W \\ I \in \mathscr{F}_{\mathrm{m}}}} w\left(H_{\phi}^{\geq}\right)=\bigcap_{\substack{w \in W \\ I \in \mathscr{F}_{\mathrm{m}}}} H_{w \phi}^{\geq} \subseteq \bigcap_{\substack{w \in W \\ I \in \mathscr{F}_{\mathrm{m} . \text { ind }}}} H_{w \phi}^{\geq}
$$

where the right hand side is closed and hence contains $\overline{\mathscr{Z}}$. Hence the inclusion " $\subseteq$ " in (b) holds. For the reverse inclusion, it suffices by Lemma 4.4(b) to show that if $z$ is in the right hand side, then $z+t \rho$ is in $\mathscr{Z}$ for all $t \in \mathbb{R}_{>0}$. But since $z \in-\overline{\mathscr{X}}$ and $-\rho$ is in the interior of $-\mathscr{C} \subseteq-\mathscr{X}$, we have $z+t \rho \in-\mathscr{X}$ and so $w(z+t \rho) \in-\mathscr{C}$ for some $w \in W$. Now for $I \in F_{\mathrm{m} \text {.ind, }}$, we have $\left\langle w z, \phi_{I}\right\rangle \geq 0$ by assumption, and we have $\left\langle t w \rho, \phi_{I}\right\rangle \geq 0$ since $t w \rho \in \mathscr{Z} \subseteq \mathbb{R}_{\geq 0} \Pi$. Hence $\left\langle w(z+t \rho), \phi_{I}\right\rangle \geq 0$, and by (a) we conclude that $w(z+t \rho) \in \mathscr{K}$. Hence $z+t \rho \in \mathscr{Z}$ for all $t>0$ as required to prove (b).

5.9. Let us say that a subset $U$ of $V$ is totally isotropic if $\langle U, U\rangle \subseteq\{0\}$. Observe that a cone in $V$ is totally isotropic if and only if it is contained in $\mathscr{Q}$ i.e. contains only isotropic vectors. Therefore, the notion of a totally isotropic subspace of $V$ in this sense coincides with the usual definition.

Lemma. (a) If $x, y \in \overline{\mathscr{Z}}$, then $\langle x, y\rangle \leq 0$.

(b) Let $n, m \in \mathbb{N}, x_{1}, \ldots, x_{n}, y_{1}, \ldots, y_{m} \in \overline{\mathscr{Z}}, x=x_{1}+\ldots+x_{n}$ and $y:=$ $y_{1}+\ldots+y_{m}$. Then $\langle x, y\rangle=0$ if and only if $\left\langle x_{i}, y_{j}\right\rangle=0$ for all $i=1, \ldots, n$ and $j=1, \ldots, m$.

(c) Let $n \in \mathbb{N}, x_{1}, \ldots, x_{n} \in \overline{\mathscr{Z}}$ and $x=x_{1}+\ldots+x_{n}$. Then $x$ is isotropic if and only if $\mathbb{R}\left\{x_{1}, \ldots, x_{n}\right\}$ is a totally isotropic subspace of $\mathbb{R} \mathscr{Z}$.

Proof. Part (a) follows directly from Proposition 3.2(c). Part (b) holds since $\langle x, y\rangle=\sum_{i, j}\left\langle x_{i}, y_{j}\right\rangle$ where $\left\langle x_{i}, y_{j}\right\rangle \leq 0$ for all $i, j$ by (a). Finally, (c) follows from (b) taking $m=n$ and $y_{i}=x_{i}$ for all $i$.

5.10. Make assumptions as in 1.4 and 3.6 but assume that $(\Phi, \Pi)$ on $(V,\langle-,-\rangle)$ satisfies 4.1(i)-(iii) and $V^{\prime}$ is finite-dimensional. Note that the radical of $\langle-,-\rangle^{\prime}$ is $\operatorname{ker}(L)$, so $\left(\Phi^{\prime}, \Pi^{\prime}\right)$ on $\left(V^{\prime},\langle-,-\rangle^{\prime}\right)$ does not necessarily satisfy 4.1)(i)-(iii).

Let $R_{W,+}=\left\{\mathbb{R}_{\geq 0} \alpha \mid \alpha \in \Phi_{+}\right\} \subseteq \operatorname{ray}\left(\mathbb{R}_{\geq 0} \Pi\right)$ and $R_{W^{\prime},+}=\left\{\mathbb{R}_{\geq 0} \alpha \mid \alpha \in\right.$ $\left.\Phi_{+}^{\prime}\right\} \subseteq \operatorname{ray}\left(\mathbb{R}_{\geq 0} \Pi^{\prime}\right)$ denote the set of rays spanned by roots in $\Phi_{+}$and $\Phi_{+}^{\prime}$ respectively, and let $R_{W, 0}=\overline{R_{W,+}} \backslash R_{W,+}$ and $R_{W^{\prime}, 0}=\overline{R_{W^{\prime},+}} \backslash R_{W^{\prime},+}$ denote the corresponding sets of limit rays (i.e limit points). By Proposition 3.6 (a), there is a $\operatorname{map} L^{\prime}: \operatorname{ray}\left(\mathbb{R}_{\geq 0} \Pi^{\prime}\right) \rightarrow \operatorname{ray}\left(\mathbb{R}_{\geq 0} \Pi\right)$, determined by $L^{\prime}\left(\mathbb{R}_{\geq 0} \alpha\right)=\mathbb{R}_{\geq 0} L(\alpha)$.

Proposition. (a) $L^{\prime}$ restricts to a surjective map $R_{W^{\prime}, 0} \rightarrow R_{W, 0}$.

(b) $L$ restricts to a surjective map $\overline{\mathscr{Z}}_{W^{\prime}} \rightarrow \overline{\mathscr{Z}}_{W}$.

Proof. Clearly, $L^{\prime}$ is continuous and it surjective by Proposition 3.6(b). So $L^{\prime}$ is a continuous surjective map between compact Hausdorff spaces and is therefore a closed map. By 1.9, $L^{\prime}$ restricts to a bijection $R_{W^{\prime},+} \rightarrow R_{W,+}$. This implies that $L^{\prime}\left(\overline{R_{W^{\prime},+}}\right)=\overline{L^{\prime}\left(R_{W^{\prime},+}\right)}=\overline{R_{W,+}}$. Note that $L^{\prime}$ preserves isotropic rays (and nonisotropic rays), so it induces a surjection from the set of isotropic rays in $\overline{R_{W^{\prime},+}}$ to the set of isotropic rays in $\overline{R_{W,+}}$. That is, by Proposition $5.3 L^{\prime}\left(R_{W^{\prime}, 0}\right)=R_{W, 0}$, proving (a). By Proposition 3.6 (c)), we have $\overline{\mathscr{Z}}=\overline{L^{\prime}\left(\mathscr{Z}_{W^{\prime}}\right)}=L^{\prime}\left(\overline{\mathscr{Z}_{W^{\prime}}}\right)$ since $L^{\prime}$ 
is closed, proving (b). (An alternative proof of (b) could be given using (a) and Theorem 5.4.)

\section{IMAGinARY CONE OF A REFLECTION SUBGROUP}

6.1. In preparation for the proof of Theorem 6.3, we state two lemmas. The first is of interest even apart from its role in the proof of the theorem. Recall that $\rho$ denotes an arbitrary but fixed element of the interior of $\mathscr{C}$.

Lemma. Suppose that $v \in \overline{\mathscr{Z}}$. Then

(a) $\overline{W v} \subseteq \overline{\mathscr{Z}} \subseteq \mathbb{R}_{\geq 0} \Pi$.

(b) $0 \leq \lambda:=\inf (\{\langle x, \rho\rangle \mid x \in \overline{W v}\})$.

(c) $\emptyset \neq M_{v}:=\{x \in \overline{W v} \mid\langle x, \rho\rangle=\lambda\}$.

(d) $M_{v} \subseteq \mathscr{K}$.

(e) $\overline{W v} \cap \mathscr{K} \neq \emptyset$.

(f) If $v$ is non-isotropic, or $v \in \mathscr{Z} \backslash\{0\}$, then $\lambda>0$.

Proof. Part (a) holds since $\overline{\mathscr{Z}}$ is $W$-invariant (since $\mathscr{Z}$ is), $\mathscr{Z} \subseteq \mathbb{R} \Pi$ and $\mathbb{R}_{\geq 0} \Pi$ is closed (being a polyhedral cone). Part (b) follows from (a) since $\left\langle\rho, \mathbb{R}_{>0} \Pi\right\rangle \subseteq \mathbb{R}_{>0}$. One may choose a sequence $\left(w_{n}\right)_{n \in \mathbb{N}}$ in $W$ with $\lim _{n \rightarrow \infty}\left\langle\rho, w_{n} v\right\rangle=\lambda$. Since $\left\{z \in \mathbb{R}_{\geq 0} \Pi \mid\langle\rho, z\rangle \leq \lambda+1\right\}$ is compact, by passing to a subsequence we may assume that the sequence $\left(w_{n} v\right)$ is convergent, say to $x \in \overline{W v}$. Then $x \in M_{v}$. This proves (c). Now let $x \in M_{v}$ be arbitrary. One may choose a sequence $\left(w_{n}\right)$ in $W$ such that $\left(w_{n} v\right)$ converges to $x$. To prove (d), it will suffice to show that $x \in-\mathscr{C}$ (for then $x \in-\mathscr{C} \cap \mathbb{R}_{\geq 0} \Pi \subseteq \mathscr{K}$ ). Suppose to the contrary that $\langle x, \alpha\rangle=c>0$ for some $\alpha \in \Pi$. We have $\langle\rho, x\rangle=\lambda$. Recall that $\langle\rho, \alpha\rangle>0$. Since $\lim _{n \rightarrow \infty} w_{n} v=x$, we may choose $n \in \mathbb{N}$ sufficiently large that both $\left\langle\rho, w_{n} v\right\rangle\langle\lambda+c\langle\rho, \alpha\rangle$ and $d:=\left\langle w_{n} v, \alpha^{\vee}\right\rangle>\frac{1}{2}\left\langle x, \alpha^{\vee}\right\rangle=c$. Then since $s_{\alpha}\left(w_{n} v\right) \in \overline{W v}$, one has

$$
\lambda \leq\left\langle\rho, s_{\alpha}\left(w_{n} v\right)\right\rangle=\left\langle\rho, w_{n} v-d \alpha\right\rangle\langle\lambda+c\langle\rho, \alpha\rangle-d\langle\rho, \alpha\rangle<\lambda,
$$

which is a contradiction proving (d). Part (e) is immediate from (c) and (d). In (f), the case in which $v \in \mathscr{Z}$ follows since there is $v^{\prime \prime} \in W v \cap \mathscr{K}$ and then $\rho\left(v^{\prime \prime}\right) \geq \rho\left(v^{\prime}\right)$ for all $v^{\prime \prime} \in W v$, hence for all $v^{\prime \prime} \in \overline{W v}$. Assume now that $\langle v, v\rangle \neq 0$ and let $x \in M_{v}$. One has $\langle y, y\rangle=\langle v, v\rangle$ for all $y \in W v$ and hence for all $y \in \overline{W v}$. In particular, $\langle x, x\rangle=\langle v, v\rangle$. If $\langle v, v\rangle \neq 0$, then $\langle x, x\rangle \neq 0$. This gives $x \in \mathbb{R}_{\geq 0} \Pi \backslash\{0\}$ and so $\lambda=\langle\rho, x\rangle>0$.

6.2. The second lemma required in our proof of Theorem 6.3 is purely technical; it follows from the theorem and Lemma 4.4 (a).

Lemma. Let $W^{\prime}$ be a finitely-generated reflection subgroup of $W$ and $z \in \mathscr{Z}_{W^{\prime}}$. Then the $W$-orbit $W z$ of $z$ is discrete and closed in $V$.

Proof. Without loss of generality, we may assume that $z=k \in \mathscr{K}_{W^{\prime}}$. It will suffice to prove the following claim: if $\left(w_{n}\right)_{n \in \mathbb{N}}$ is a sequence in $W$ such that the sequence $\left(w_{n} z\right)_{n}$ converges in $V$, say to $x$, then there is a subsequence $\left(w_{n_{m}}\right)_{m \in \mathbb{N}}$ such that $w_{n_{m}} z=x$ for all sufficiently large $m$. We will show below equivalently that after passing to a suitable subsequence of $\left(w_{n}\right)$, we have $w_{n} z=x$ for all $n$.

Now $k$ lies in some face of the polyhedral cone $\mathbb{R}_{\geq 0} \Pi_{W^{\prime}}$, say that corresponding to the facial subset $I^{\prime} \subseteq S^{\prime}$ of $S^{\prime}:=\chi\left(W^{\prime}\right)$. By Lemma 3.4(a), we have $k \in$ $\mathbb{R}_{\geq 0} \Pi_{W_{I^{\prime}}^{\prime}} \cap \mathscr{K}_{W^{\prime}}=\mathscr{K}_{W_{I^{\prime}}^{\prime}}$. If the Lemma holds with $W^{\prime}$ replaced by $W_{I^{\prime}}^{\prime}$, it holds for $W^{\prime}$. Hence we may and do assume without loss of generality that $I^{\prime}=S^{\prime}$ i.e. 
$k$ is a point of the relative interior of the cone $\mathbb{R} \Pi_{W^{\prime}}$. It follows that there is an expression $k=\sum_{\alpha \in \Pi_{W^{\prime}}} c_{\alpha} \alpha$ with all $c_{\alpha}>0$, since $\Pi_{W^{\prime}}$ is a set of representatives of the extreme rays of this cone. Let $\epsilon=\min \left(\left\{c_{\alpha} \mid \alpha \in \Pi_{W^{\prime}}\right\}\right)>0$. For any $w \in W$, we write $w=w^{\prime \prime} w^{\prime}$ where $w^{\prime} \in W^{\prime}$ and $w^{\prime \prime} \in W$ satisfies $N\left(w^{\prime \prime}-1\right) \cap W^{\prime}=\emptyset$ i.e. $w^{\prime \prime}$ is the unique element of minimum length in the coset $w W^{\prime}$. Using Lemma 1.13.(c), write $w^{\prime} k=\sum_{\alpha \in \Pi^{\prime}}\left(c_{\alpha}+d_{w^{\prime}, \alpha}\right) \alpha$ where all $d_{w^{\prime}, \alpha} \geq 0$.

Note that the sequence $\left(\left\langle\rho, w_{n} k\right\rangle\right)_{n}$ is bounded above, say $\left\langle\rho, w_{n} k\right\rangle \leq M$ where $M \in \mathbb{R}_{\geq 0}$. We have

$$
\begin{aligned}
M & \geq\left\langle\rho, w_{n} k\right\rangle=\left\langle\rho, w_{n}^{\prime \prime} w_{n}^{\prime} k\right\rangle=\left\langle\rho, \sum_{\alpha \in \Pi^{\prime}}\left(c_{\alpha}+d_{w_{n}^{\prime}, \alpha}\right) w_{n}^{\prime \prime} \alpha\right\rangle \\
& \geq \epsilon \max \left(\left\{\left\langle\rho, w_{n}^{\prime \prime} \alpha\right\rangle \mid \alpha \in \Pi_{W^{\prime}}\right\}\right)
\end{aligned}
$$

where all $w_{n}^{\prime \prime}(\alpha) \in \Phi_{+}$. Hence $\left\langle\rho, w_{n}^{\prime \prime} \alpha\right\rangle \leq M / \epsilon$ for all $n \in \mathbb{N}$ and all $\alpha \in \Pi_{W^{\prime}}$. By Lemma 4.3 (e), there are only finitely many possibilities for the $\Pi_{W^{\prime}}$-indexed families $\left(w_{n}^{\prime \prime} \alpha\right)_{\alpha \in \Pi_{W^{\prime}}}$ for $n \in \mathbb{N}$. Replacing $\left(w_{n}\right)$ by a subsequence, we may assume that the sequence of families $\left(w_{n}^{\prime \prime} \alpha\right)_{\alpha \in \Pi_{W^{\prime}}}$ for $n \in \mathbb{N}$ is constant, so $\left(w_{n}^{\prime \prime} \alpha\right)_{\alpha \in \Pi_{W^{\prime}}}=$ $\left(w_{0}^{\prime \prime} \alpha\right)_{\alpha \in \Pi_{W^{\prime}}}$ for all $n \in \mathbb{N}$. Then

$$
\begin{aligned}
w_{n} k & =\sum_{\alpha \in \Pi^{\prime}}\left(c_{\alpha}+d_{w_{n}^{\prime}, \alpha}\right) w_{n}^{\prime \prime} \alpha=\sum_{\alpha \in \Pi^{\prime}}\left(c_{\alpha}+d_{w_{n}^{\prime}, \alpha}\right) w_{0}^{\prime \prime} \alpha \\
& =w_{0}^{\prime \prime} \sum_{\alpha \in \Pi^{\prime}}\left(c_{\alpha}+d_{w_{n}^{\prime}, \alpha}\right) \alpha=w_{0}^{\prime \prime} w_{n}^{\prime} k
\end{aligned}
$$

It follows that the sequence $w_{n}^{\prime} k$ converges to $w_{0}^{\prime \prime-1} x$ and in particular, the sequence $\left\langle\rho, w_{n}^{\prime} k-k\right\rangle$ for $n \in \mathbb{N}$ is bounded above. Recall that $\rho$ is an interior point of $\mathscr{C}_{W^{\prime}}$, by 1.10 (e). By 4.4.1 applied to $W^{\prime}$, we see that $w_{n}^{\prime}(k)-k$ has a constant subsequence. Passing to an appropriate subsequence of $\left(w_{n}\right)$ yet again, we may therefore assume that $w_{n}^{\prime} k$ is constant. Hence $w_{n}^{\prime} k=w_{0}^{\prime \prime-1} x$ for all $n$, and $w_{n} k=x$ for all $n$ as required to complete the proof.

6.3. The following is a main result of this work.

Theorem. Let $W^{\prime}$ be a reflection subgroup of $W$. Then $\mathscr{Z}_{W^{\prime}} \subseteq \mathscr{Z}_{W}$.

Proof. Using 3.3 (b), assume without loss of generality that $W^{\prime}$ is finitely generated. It is clear from Theorem 5.1 and Lemma $3.1(\mathrm{a})$, that $\overline{\mathscr{Z}_{W}} \subseteq \overline{\mathscr{Z}_{W}}$. Let $z \in \mathscr{Z}_{W^{\prime}}$. Then $z \in \overline{\mathscr{Z}}$, so by Lemma 6.1 there is a sequence $\left(w_{n}\right)_{n \in \mathbb{N}}$ in $W$ such that the sequence $\left(w_{n} z\right)$ converges to an element $x \in \mathscr{K}$. By Lemma 6.2, $w_{n} z=x \in \mathscr{K}$ for all but finitely many $n$. Hence $z=w_{n}^{-1} x \in \mathscr{Z}$ for some $n$, as required.

6.4. The final result in this section refines Proposition 2.6.

Corollary. Assume that $W^{\prime \prime}$ is a facial subgroup of $W$ and $W^{\prime}$ is a finitely generated reflection subgroup of $W$. Then $W^{\prime \prime \prime}:=W^{\prime} \cap W^{\prime \prime}$ is a finitely-generated reflection subgroup of $W$ and $\mathscr{Z}_{W^{\prime}} \cap \mathscr{Z}_{W^{\prime \prime}}=\mathscr{Z}_{W^{\prime \prime \prime}}$.

Proof. Note that $W^{\prime \prime \prime}$ is a finite rank reflection subgroup by 2.6. Assume first that $W^{\prime \prime}$ is standard facial, say $W^{\prime \prime}=W_{J}$ for facial $J \subseteq S$. Then $\chi\left(W^{\prime \prime}\right)=J^{\prime}:=$ $\chi\left(W^{\prime}\right) \cap W_{J}$ by 2.6. By Theorem 6.3. we have $\mathscr{Z}_{W^{\prime \prime \prime}} \subseteq \mathscr{Z}_{W^{\prime}} \cap \mathscr{Z}_{W_{J}}$. For the reverse 
inclusion, note that

$$
\begin{aligned}
\mathscr{Z}_{W^{\prime}} \cap \mathscr{Z}_{W_{J}} & \subseteq\left(\mathscr{Z}_{W^{\prime}} \cap \mathbb{R}_{\geq 0} \Pi_{W^{\prime}}\right) \cap \mathbb{R}_{J} \\
& =\mathscr{Z}_{W^{\prime}} \cap \mathbb{R}_{\geq 0} \Pi_{W_{J^{\prime}}^{\prime}}=\mathscr{Z}_{W_{J^{\prime}}^{\prime}} .
\end{aligned}
$$

Here, we use 2.6(a) to get the first equality, and Lemma 3.4 (a) applied to $W^{\prime}$ to get the second (recalling $J^{\prime}$ is facial in $\chi\left(W^{\prime}\right)$ by 2.6). This proves the Corollary in the special case that $W^{\prime \prime}$ is a standard facial subgroup of $W$. The case of a general facial subgroup $W^{\prime \prime}$ reduces easily to the special case just treated by writing $W^{\prime \prime}=w W_{J} w^{-1}$ for some facial $J \subseteq S$ and using Lemma 3.2 (e).

\section{Action on the Closed imaginary Cone}

7.1. The following simple fact will prove useful.

Lemma. Suppose that $\Phi$ is irreducible infinite but not of affine type. If $\alpha \in \mathbb{R}_{\geq 0} \Pi$ is non-zero, then $\alpha \not \perp \Pi$ and $\operatorname{aff}(W \alpha)=\mathbb{R}(W \alpha)=\mathbb{R} \Pi$.

Proof. Write $\alpha=\sum_{\gamma \in \Gamma} c_{\gamma} \gamma$ for some (finite) $\Gamma \subseteq \Pi$ where all $c_{\gamma}>0$. If $\Gamma \subsetneq \Pi$, then, by irreducibility of $\Pi$, there is some $\beta \in \Pi \backslash \Gamma$ with $\langle\Gamma, \beta\rangle \neq 0$ and then $\langle\beta, \alpha\rangle<0$ also. Otherwise, $\Gamma=\Pi$ and so $\langle\Pi, \alpha\rangle \neq\{0\}$ since $\Pi$ is not of affine type. Hence $\alpha \not \perp \Pi$. By Lemma $1.13(\mathrm{~d}), \mathbb{R}(W \alpha) \supseteq$ aff $(W \alpha)=\alpha+\mathbb{R} \Pi=\mathbb{R} \Pi \supseteq \mathbb{R}(W \alpha)$ and equality must hold throughout.

7.2. Recall from Section 5 the definitions of the set ray $(V)$ of rays of $V$ (as topologized in 5.3 and its subsets $R_{0} \supseteq R_{0}^{\prime} \supseteq R_{0}^{\prime \prime}$ of isotropic rays. The analogously defined subsets for a reflection subgroup $W^{\prime}$ are denoted $R_{W^{\prime}, 0} \supseteq R_{W^{\prime}, 0}^{\prime} \supseteq R_{W^{\prime}, 0}^{\prime \prime}$.

Lemma. Suppose that $\Phi$ is irreducible and not of affine type and that $\alpha \in \mathscr{Z} \backslash\{0\}$. Let $W^{\prime}$ be a non-trivial reflection subgroup of $W$.

(a) There exists $\beta \in W \alpha \cap-\mathscr{C}_{W^{\prime}}$ such that $\beta \not \perp \Pi_{W^{\prime}}$.

(b) Assume further that $W^{\prime}$ is infinite irreducible and let $\beta$ be as in (a). Then there exists $\epsilon>0$ such that $\Psi:=\left\{\gamma \in \Phi_{W^{\prime},+} \mid\left\langle\beta, \gamma^{\vee}\right\rangle<-\epsilon\right\}$ is infinite.

(c) Let assumptions be as in (b). Then there is a sequence $\left(\gamma_{n}\right)_{n \in \mathbb{N}}$ of distinct roots in $\Psi$ such that $\left(\mathbb{R}_{\geq 0} \gamma_{n}\right)$ converges in $\operatorname{ray}(V)$ to a ray $\mathbb{R}_{\geq 0} \gamma$ in $R_{W^{\prime}, 0}$. Further, for any such sequence $\left(\gamma_{n}\right),\left(\mathbb{R}_{\geq 0} s_{\gamma_{n}}(\beta)\right)_{n \in \mathbb{N}}$ then necessarily converges in $\operatorname{ray}(V)$ to $\mathbb{R}_{\geq 0} \gamma$.

Proof. First we prove (a). Without loss of generality, assume that $\alpha \in \mathscr{K} \subseteq-\mathscr{C}_{W^{\prime}}$. If $\alpha \not \perp \Pi_{W^{\prime}}$, then $\beta:=\alpha$ satisfies the requirements of (a). Henceforward, suppose that $\alpha \in \mathscr{K} \cap \Pi_{W^{\prime}}^{\perp}$. Set $\Delta:=\Pi \cap \alpha^{\perp}$. By Lemma 7.1, we have $\Delta \neq \Pi$.

For each $\gamma \in \Pi_{W^{\prime}}$, choose a finite set $\Gamma_{\gamma} \subseteq \Pi$ and scalars $c_{\gamma, \delta}>0$ for $\delta \in \Gamma_{\gamma}$ such that $\gamma=\sum_{\delta \in \Gamma_{\gamma}} c_{\gamma, \delta} \delta$. We have $0=\langle\gamma, \alpha\rangle=\sum_{\delta \in \Gamma_{\gamma}} c_{\gamma, \delta}\langle\delta, \alpha\rangle$ where each $\langle\delta, \alpha\rangle \leq 0$ since $\alpha \in-\mathscr{C}$ and $\langle\delta, \alpha\rangle<0$ if $\delta \in \Pi \backslash \Delta$. This implies that $\Gamma_{\gamma} \subseteq \Delta$ and hence $\Gamma:=\cup_{\gamma \in \Pi_{W^{\prime}}} \Gamma_{\gamma} \subseteq \Delta$. Note that $\Pi_{W^{\prime}} \subseteq \mathbb{R} \Gamma$. Also, $\Gamma \neq \emptyset$ since $\Pi_{W^{\prime}} \neq \emptyset$ by the assumed non-triviality of $W^{\prime}$.

Using connectedness of the Coxeter graph of $W$, we may choose $p \in \mathbb{N}$ and a sequence $\delta_{0}, \delta_{1}, \ldots, \delta_{p}$ of simple roots with $\delta_{0} \in \Pi \backslash \Delta, \delta_{p} \in \Gamma$ and $\left\langle\delta_{i-1}, \delta_{i}\right\rangle \neq 0$ for $i=1, \ldots, p$. Suppose $p$ and the sequence is chosen so $p$ is minimal amongst all such sequences. Then $p \geq 1, \delta_{1}, \ldots, \delta_{p} \in \Delta$ and $\delta_{0}, \ldots, \delta_{p-1} \notin \Gamma$. Moreover, if $0 \leq i \leq p-2$, then $\delta_{i}$ is not joined in the Coxeter graph of $W$ to any element of $\Gamma$ and so $\delta_{i} \perp \Pi_{W^{\prime}}$. 
Set $\beta:=s_{\delta_{p-1}} \ldots s_{\delta_{0}}(\alpha) \in W \alpha$. By Lemma 1.13(a), $\beta=\alpha+c \tau$ where $c=$ $-\left\langle\alpha, \delta_{0}^{\vee}\right\rangle>0$ (since $\delta_{0} \notin \Delta$ ) and $\tau:=s_{\delta_{p-1}} \ldots s_{\delta_{1}}\left(\delta_{0}\right)$. Using Lemma 1.16(a), one has $\beta=\alpha+b_{0} \delta_{0}+\ldots+b_{p-1} \delta_{p-1}$ for some $b_{1}, \ldots, b_{p-1} \in \mathbb{R}_{>0}$. Now for $\gamma \in \Pi_{W^{\prime}}$,

$$
\langle\beta, \gamma\rangle=\left\langle\alpha+\sum_{i=0}^{p-1} b_{i} \delta_{i}, \gamma\right\rangle=b_{p-1}\left\langle\delta_{p-1}, \gamma\right\rangle=\sum_{\delta \in \Gamma_{\gamma}} b_{p-1} c_{\gamma, \delta}\left\langle\delta_{p-1}, \delta\right\rangle .
$$

Since $\delta_{p-1} \notin \Gamma$ and $\Gamma \supseteq \Gamma_{\gamma}$, it follows that $\langle\beta, \gamma\rangle \leq 0$ and $\beta \in-\mathscr{C}_{W^{\prime}}$. Moreover, one has $\delta_{p} \in \Gamma$, so $\delta_{p} \in \Gamma_{\gamma}$ for some $\gamma \in \Pi_{W^{\prime}}$. Then $\langle\beta, \gamma\rangle \leq b_{p-1} c_{\gamma, \delta_{p}}\left\langle\delta_{p-1}, \delta_{p}\right\rangle<0$ and so $\beta \notin \Pi_{W^{\prime}}^{\perp}$. This proves (a).

For the proof of $(\mathrm{b})$, choose $\gamma_{0} \in \Pi_{W^{\prime}}$ with $\epsilon:=-\left\langle\beta, \gamma_{0}^{\vee}\right\rangle>0$. Let $W^{\prime \prime}:=$ $W_{\Pi_{W^{\prime}} \backslash\left\{\gamma_{0}\right\}}$. Let $\Psi^{\prime}:=\Phi_{W^{\prime},+} \backslash \Phi_{W^{\prime \prime},+}$ which is infinite by Lemma $1.20(\mathrm{~b})$. We claim that $\Psi^{\prime} \subseteq \Psi$ i.e. $\left\langle\beta, \tau^{\vee}\right\rangle<-\epsilon$ for all $\tau \in \Psi^{\prime}$. This may be regarded as a statement purely in terms of inner products in the root system $\Phi_{W^{\prime}}$, for the proof of which we may assume $\Pi_{W^{\prime}}$ is linearly independent By Lemma 2.4 each element $\tau$ of $\Psi^{\prime}$ can be written in the form $\tau=\sum_{\gamma \in \Pi_{W^{\prime}}} c_{\gamma} \gamma$ where all $c_{\gamma} \geq 0$ and $c_{\gamma_{0}}>0$. By 1.4 and a result of Brink (see [3, Lemma (3.3)]), one may choose the $c_{\gamma}$ so $c_{\gamma_{0}} \geq 1$. Hence $\left\langle\beta, \tau^{\vee}\right\rangle=\left\langle\beta, \sum_{\gamma \in \Pi_{W^{\prime}}} c_{\gamma} \gamma^{\vee}\right\rangle \leq c_{\gamma_{0}}\left\langle\beta, \gamma_{0}^{\vee}\right\rangle<-\epsilon$. This proves (b).

Finally, we prove (c). Since $\Psi$ is infinite, there exists an infinite sequence $\left(\gamma_{n}\right)_{n \in \mathbb{N}}$ of distinct elements of $\Psi$. Since $W$ is of finite rank, $\rho\left(\gamma_{n}\right) \rightarrow \infty$ as $n \rightarrow \infty$ by (4.4.1). Since $\operatorname{ray}(V)$ is compact, by passing to a subsequence if necessary, we may assume this sequence converges in $\operatorname{ray}(V)$ to a ray $\mathbb{R}_{\geq 0} \gamma \in R_{W^{\prime}, 0}$, where $\gamma \in \mathbb{R}_{\geq 0} \Pi \backslash\{0\}$. Now let $\left(\gamma_{n}\right)$ be any sequence of distinct roots in $\Psi$ with $\mathbb{R}_{\geq 0} \gamma_{n} \rightarrow \mathbb{R}_{\geq 0} \gamma$. Then $\left\langle\rho, \gamma_{n}\right\rangle^{-1} \gamma_{n} \rightarrow\langle\rho, \gamma\rangle^{-1} \gamma$ and $\left\langle\rho, \gamma_{n}\right\rangle \rightarrow \infty$ as $n \rightarrow \infty$. Now $s_{\gamma_{n}}(\beta)=\beta-$ $\left\langle\beta, \gamma_{n}^{\vee}\right\rangle \gamma_{n}$. Let $c_{n}:=-\left\langle\beta, \gamma_{n}^{\vee}\right\rangle^{-1}\left\langle\rho, \gamma_{n}\right\rangle^{-1}$. Since $\epsilon<-\left\langle\beta, \gamma_{n}^{\vee}\right\rangle$ for all $n, c_{n}>0$ and $c_{n} \rightarrow 0$ as $n \rightarrow \infty$. One has $\mathbb{R}_{\geq 0} s_{\gamma_{n}}(\beta)=\mathbb{R}_{\geq 0} \delta_{n}$ where $\delta_{n}:=c_{n} s_{\gamma_{n}}(\beta)=$ $c_{n} \beta_{n}+\left\langle\rho, \gamma_{n}\right\rangle^{-1} \gamma_{n}$. Clearly, as $n \rightarrow \infty, \delta_{n} \rightarrow\langle\rho, \gamma\rangle^{-1} \gamma$ and so $\mathbb{R}_{\geq 0} s_{\gamma_{n}}(\beta) \rightarrow \mathbb{R}_{\geq 0} \gamma$ as required.

Remarks. (1) The proofs above of Lemma 7.1 and (a)-(b) hold in the framework in Sections 1 , 3 (in particular, $W$ need not be of finite rank). However, if $W$ is finite or of locally finite type, then $\mathscr{Z}=\{0\}$, so no $\alpha$ as in the statement of the above lemma exists.

(2) In the case $W^{\prime}$ is infinite dihedral, a simpler proof of (b) is as follows. Write $\Pi_{W^{\prime}}=\left\{\delta, \delta^{\prime}\right\}$. Then $\Phi_{W^{\prime},+} \backslash \Pi_{W^{\prime}} \subseteq \Psi$ since, as is well known and easily checked, for $\tau \in \Phi_{W^{\prime},+} \backslash \Pi_{W^{\prime}}$, one has $\tau=c \delta+d \delta^{\prime}$ where $c, d \geq 1$.

7.3. If $w \in W$ and $\mathscr{Z} \neq 0$, then $w$ has an eigenvector $\alpha$ in $\overline{\mathscr{Z}}$ with strictly positive eigenvalue equal to the spectral radius of $w$ on $\mathbb{R} \mathscr{Z}$, by Perron-Frobenius theory (see [48, 43]).

Lemma. Let $\alpha \in \overline{\mathscr{Z}}$ be an eigenvector of $w \in W$ with (real) eigenvalue $\lambda$. So $\alpha \neq 0, w \alpha=\lambda \alpha$ and $\lambda>0$. Let $V_{w, \lambda}:=\{\gamma \in V \mid w(\gamma)=\lambda \gamma\}$ be the $\lambda$-eigenspace of $w$ on $V$

(a) If $\lambda \neq 1$, then $V_{w, \lambda} \cap \overline{\mathscr{Z}}$ is a totally isotropic subset of $V$.

(b) If $\lambda>1$, then $\left\langle\rho, w^{n} \alpha\right\rangle \rightarrow \infty$ and $w^{-n} \alpha \rightarrow 0$ as $n \rightarrow \infty$. Similarly, if $\lambda<1$, then $w^{n} \alpha \rightarrow 0$ and $\left\langle\rho, w^{-n} \alpha\right\rangle \rightarrow \infty$ as $n \rightarrow \infty$.

(c) If $\alpha^{\prime}$ and $\alpha^{\prime \prime}$ are linearly independent eigenvectors of $w$ in $\operatorname{ri}(\mathscr{Z})$ with corresponding eigenvalues $\lambda^{\prime}$ and $\lambda^{\prime \prime}$, then $\lambda^{\prime}=\lambda^{\prime \prime}$ and there is an eigenvector $\alpha^{\prime \prime} \in \operatorname{rb}(\mathscr{Z})$ of $w$ with eigenvalue $\lambda^{\prime}$. 
Proof. One has $\lambda \neq 0$ since $w$ acts invertibly on $\mathbb{R} \mathscr{Z}$ and $\lambda \nless 0$ since $\overline{\mathscr{Z}}$ is salient. Hence $\lambda>0$. Assume $\lambda \neq 1$. If $\beta, \gamma \in V_{w, \lambda}$, then $\langle\beta, \gamma\rangle=\langle w \beta, w \gamma\rangle=\lambda^{2}\langle\beta, \gamma\rangle$ and so $\langle\beta, \gamma\rangle=0$ since $\lambda^{2} \neq 1$. Part (a) follows readily. Suppose now that $\lambda>1$. Then $\langle\rho, \alpha\rangle \neq 0$ since $\alpha \neq 0,\left\langle\rho, w^{n} \alpha\right\rangle=\lambda^{n}\langle\rho, \alpha\rangle \rightarrow \infty$ and $\left\langle\rho, w^{-n} \alpha\right\rangle=$ $\lambda^{-n}\langle\rho, \alpha\rangle \rightarrow 0$ as $n \rightarrow \infty$. Hence $w^{-n} \alpha \rightarrow 0$ by Remark A.11. This proves the first part of (b), and the second follows from it. Part (c) is a special case of 48, Lemma 4.1].

7.4. For $\alpha \in \overline{\mathscr{Z}} \backslash\{0\}$, set $E_{\alpha}:=\left\{\mathbb{R}_{\geq 0} \beta \mid \beta \in W \alpha\right\} \subseteq \operatorname{ray}(V)$ and let $E_{\alpha}^{\prime} \subseteq \overline{E_{\alpha}}$ be the set of limit points of $E_{\alpha}$ in $\operatorname{ray}(V)$. One has $\overline{E_{\alpha}^{\prime}}=E_{\alpha}^{\prime}$.

Lemma. If $\alpha \in(\overline{\mathscr{Z}} \backslash\{0\}) \cap(\mathscr{Z} \cup \mathscr{Q})$, then $E_{\alpha}^{\prime} \subseteq Q$.

Proof. Note that if two rays $\mathbb{R}_{\geq 0} x(\alpha), \mathbb{R}_{\geq 0} y(\alpha)$, where $x, y \in W$ and $\alpha \in \overline{\mathscr{Z}} \backslash\{0\}$, are equal, then $\alpha$ is an eigenvector in $\overline{\mathscr{Z}}$ of $x^{-1} y$. Suppose that $\mathbb{R}_{\geq 0} \beta, 0 \neq \beta \in$ $\mathbb{R}_{\geq 0} \Pi$, is a limit ray of $E_{\alpha}$. Then there is a sequence $\left(w_{n}\right)_{n \in \mathbb{N}}$ of elements of $W$ such that the rays $\mathbb{R}_{\geq 0} w_{n} \alpha$ in $\overline{\mathscr{Z}}$ are pairwise distinct and converge in $\operatorname{ray}(V)$ to $\mathbb{R}_{\geq 0} \beta$. That is, $\gamma_{n}:=\left\langle\rho, w_{n} \alpha\right\rangle^{-1} w_{n} \alpha \rightarrow\langle\rho, \beta\rangle^{-1} \beta$ as $n \rightarrow \infty$ in $V$. If $\alpha$ is isotropic, then so is each $w_{n} \alpha$ and so $\beta$ is isotropic as required. If $\alpha \in \mathscr{Z}$, then, since $W \alpha \cap-\mathscr{C} \neq \emptyset$, 4.4.1 implies that for any $\eta>0$, there are only finitely many $\gamma \in W \alpha$ with $\langle\gamma, \rho\rangle<\eta$. Hence as $n \rightarrow \infty,\left\langle\rho, w_{n} \alpha\right\rangle \rightarrow \infty$. Therefore $\left\langle\gamma_{n}, \gamma_{n}\right\rangle=\left\langle\rho, w_{n} \alpha\right\rangle^{-2}\left\langle w_{n} \alpha, w_{n} \alpha\right\rangle \rightarrow 0$ since $\left\langle w_{n} \alpha, w_{n} \alpha\right\rangle=\langle\alpha, \alpha\rangle$. But also $\left\langle\gamma_{n}, \gamma_{n}\right\rangle \rightarrow\langle\rho, \beta\rangle^{-2}\langle\beta, \beta\rangle$, so $\beta$ is isotropic as claimed.

7.5. The main consequence of the next result is stated as Theorem 7.6 .

Theorem. Assume $W$ is irreducible.

(a) If $\alpha \in \mathscr{Z} \backslash\{0\}$, then $R_{0} \subseteq \overline{E_{\alpha}}$.

(b) If $\alpha \in \overline{\mathscr{Z}} \backslash\{0\}$, then $\overline{\mathscr{Z}}=\operatorname{cl}\left(\operatorname{conv}\left(\bigcup E_{\alpha}\right)\right)=\operatorname{conv}\left(\operatorname{cl}\left(\bigcup E_{\alpha}\right)\right)$.

Proof. If $W$ is finite, then (a)-(b) hold vacuously and if $W$ is affine, they hold trivially since $\alpha$ spans the isotropic ray $\mathbb{R}_{>_{0}} \alpha=\mathscr{K}=\mathscr{Z}=\overline{\mathscr{Z}}$ and is fixed by $W$. Henceforward assume $W$ is of indefinite type. For the proof of (a), fix $\alpha \in \mathscr{Z} \backslash\{0\}$. Let $W^{\prime}$ be any infinite dihedral reflection subgroup of $W$. By Lemma 7.2 (c), there is $w \in W$ such that the closure in $\operatorname{ray}(V)$ of the $W^{\prime}$-orbit of $\mathbb{R}_{\geq 0} w \alpha$ contains a point of $R_{W^{\prime}, 0}$. By Corollary 5.5(d), the closure of the $W$-orbit $E_{\alpha}$ of $\mathbb{R}_{\geq 0} \alpha$ contains $R_{W^{\prime}, 0}$ for all infinite dihedral subgroups $W^{\prime}$ of $W$. That is, $R_{0}^{\prime} \subseteq \bar{E}_{\alpha}$ and hence $R_{0}=\overline{R_{0}^{\prime}} \subseteq \bar{E}_{\alpha}$ by Theorem 5.6(b). This proves the assertion of (a).

For (b), let $\alpha \in \overline{\mathscr{Z}} \backslash\{0\}$. By Lemma 7.1 we have $\mathbb{R}(W \alpha)=\mathbb{R} \Pi$. One may therefore choose $\Gamma \subseteq W \alpha \subseteq \overline{\mathscr{Z}}$ which is a basis for $\mathbb{R} \Pi$. Then $\mathbb{R}_{>0} \Gamma \subseteq$ $\operatorname{ri}\left(\mathbb{R}_{\geq 0} \Gamma\right) \subseteq \operatorname{ri}(\overline{\mathscr{Z}})=\operatorname{ri}(\mathscr{Z})$. Let $\alpha^{\prime} \in \mathbb{R}_{>0} \Gamma$. By (a) and Theorem 5.3(a), it follows that $\overline{\mathscr{Z}} \supseteq \operatorname{conv}\left(\cup \bar{E}_{\alpha^{\prime}}\right) \supseteq \operatorname{conv}\left(\cup R_{0}\right)=\overline{\mathscr{Z}}$. By Lemma A.12(b) and (d) this gives $\overline{\mathscr{Z}}=\operatorname{conv}\left(\operatorname{cl}\left(\bigcup_{w \in W} \mathbb{R}_{\geq 0} w\left(\alpha^{\prime}\right)\right)\right)=\operatorname{cl}\left(\operatorname{conv}\left(\bigcup_{w \in W} \mathbb{R}_{\geq 0} w\left(\alpha^{\prime}\right)\right)\right)$. Since $\alpha^{\prime} \in$ $\operatorname{conv}\left(\cup_{w \in W} \mathbb{R}_{\geq 0} w \alpha\right)$, this implies that

$$
\overline{\mathscr{Z}} \supseteq \operatorname{cl}\left(\operatorname{conv}\left(\bigcup_{w \in W} \mathbb{R}_{\geq 0} w(\alpha)\right)\right) \supseteq \operatorname{cl}\left(\operatorname{conv}\left(\bigcup_{w \in W} \mathbb{R}_{\geq 0} w\left(\alpha^{\prime}\right)\right)\right)=\overline{\mathscr{Z}} .
$$

This establishes that $\overline{\mathscr{Z}}=\operatorname{cl}\left(\operatorname{conv}\left(\bigcup E_{\alpha}\right)\right)$. By Lemma A.12, $\operatorname{cl}\left(\operatorname{conv}\left(\bigcup E_{\alpha}\right)\right)=$ $\overline{\mathscr{Z}}=\operatorname{conv}\left(\operatorname{cl}\left(\bigcup E_{\alpha}\right)\right)$, completing the proof of (b). 
7.6. For finite $W, \mathscr{Z}=\{0\}$ is the only non-empty $W$-invariant cone contained in $\mathbb{R}_{\geq 0} \Pi$, since any such cone is contained in $\mathbb{R}_{\geq 0} \Pi \cap w_{S}\left(\mathbb{R}_{\geq 0} \Pi\right)=\mathbb{R}_{\geq 0} \Pi \cap-\mathbb{R}_{\geq 0} \Pi=$ $\{0\}$ where $w_{S}$ is the longest element. For irreducible infinite $W$, one has instead:

Theorem. Suppose that $W$ is irreducible and infinite. Then $\overline{\mathscr{Z}}$ is the unique nonzero $W$-invariant closed pointed cone contained in $\mathbb{R}_{\geq 0} \Pi$.

Proof. It has already been shown that $\overline{\mathscr{Z}}$ has the properties listed. Let $C$ be any cone with these properties, and fix $\alpha \in C$ with $\alpha \neq 0$. Then $C \subseteq \cap_{w \in W} w\left(\mathbb{R}_{\geq_{0}} \Pi\right)=$ $\mathscr{Y}=\overline{\mathscr{Z}}$ by Theorem 5.1. Hence $\alpha \in \overline{\mathscr{Z}} \backslash\{0\}$. Therefore, by Proposition 7.5 $C \supseteq \operatorname{cl}\left(\operatorname{conv}\left(\cup_{w \in W} \mathbb{R}_{\geq 0} w \alpha\right)\right)=\overline{\mathscr{Z}}$.

7.7. If $(W, S)$ is affine or dihedral of indefinite type, then $\overline{\mathscr{Z}}$ has either one or two extreme rays, which form a single $W$-orbit. This behavior is exceptional amongst the infinite irreducible Coxeter groups.

Lemma. Suppose that $(W, S)$ is irreducible of indefinite type and $|S| \geq 3$. Then

(a) The $W$-orbit of any ray contained in $\overline{\mathscr{Z}}$ is infinite.

(b) $\overline{\mathscr{Z}}$ is not a polyhedral cone.

Proof. Suppose that $0 \neq \alpha \in \overline{\mathscr{Z}}$ and there are only finitely many distinct rays in the $W$-orbit of $\mathbb{R}_{\geq 0} \alpha$, say $\mathbb{R}_{\geq 0} \alpha_{i}$ for $i=1, \ldots, n$. Let $C:=\mathbb{R}_{\geq 0} \Gamma$, where $\Gamma=\left\{\alpha_{1}, \ldots, \alpha_{n}\right\}$, be the polyhedral cone spanned by these rays. Then $C$ has the properties listed in Theorem 7.6, so $C=\overline{\mathscr{Z}}$. Let $W^{\prime}$ be the pointwise stabilizer in $W$ of the set $\left\{\mathbb{R}_{\geq 0} \alpha_{i} \mid i=1, \ldots, n\right\}$ of rays, so $W^{\prime}$ is a normal subgroup of $W$ of finite index. Each $w \in W^{\prime}$ has each element of $\Gamma$ as an eigenvector. Since $\Gamma$ spans $\mathbb{R} \Gamma=\mathbb{R} \mathscr{Z}=\mathbb{R} \Pi$ and $W$ acts faithfully on $\Phi \subseteq \mathbb{R} \Pi$, it follows that $W^{\prime}$ is abelian. By Remark 4.5 $(2),(W, S)$ is affine, a contradiction. Now (b) follows from (a) since if $\overline{\mathscr{Z}}$ is polyhedral, the $W$-orbit of an extreme ray of $\overline{\mathscr{Z}}$ is contained in the (finite) set of extreme rays of $\overline{\mathscr{Z}}$.

7.8. Let $\mathrm{R}_{\text {ext }}$ (resp., $\mathrm{R}_{\text {exp }}$ ) denote the set of extreme (resp., exposed) rays of $\overline{\mathscr{Z}}$ and $Z:=\operatorname{ray}(\mathscr{Z})$ denote the set of rays of $\mathscr{Z}$, so the set of rays of $\overline{\mathscr{Z}}$ is ray $(\overline{\mathscr{Z}})=\bar{Z}$ (where the latter closure is taken in $\operatorname{ray}(V)$ ). Note that

$$
R_{0} \subseteq \bar{Z} \cap Q
$$

by Theorem 5.4 and Proposition 5.3 and

$$
\mathrm{R}_{\text {exp }} \subseteq \mathrm{R}_{\text {ext }} \subseteq \overline{\mathrm{R}_{\text {exp }}}=\overline{\mathrm{R}_{\text {ext }}} \subseteq \bar{Z}
$$

for general reasons (see Lemma A.11(b)).

Proposition. Let $W$ be arbitrary. Then

(a) $\overline{\mathscr{Z}}$ is the set consisting of (0 and) all sums $y_{1}+\ldots+y_{n}$ where $n>0$ and all $y_{i} \in \cup \mathrm{R}_{\text {ext }}$.

(b) $\mathscr{Q} \cap \overline{\mathscr{Z}}$ is the set consisting of (0 and) all sums $y_{1}+\ldots+y_{n}$ where $n>0$, all $y_{i} \in \cup \mathrm{R}_{\text {ext }}$ and $\left\langle y_{i}, y_{j}\right\rangle=0$ for $i, j=1, \ldots, n$.

Proof. Part (a) is a general property of the extreme rays of a closed salient cone (see Lemma A.11(b)) and (b) follows from (a) and Lemma 5.9 
7.9. $\quad$ For $\beta \in \Phi_{+}$, let $R_{\beta, 0}:=\overline{R_{\beta,+}} \backslash R_{\beta,+} \subseteq R_{0}$ where $R_{\beta,+}:=\left\{\mathbb{R}_{\geq 0} \gamma \mid \gamma \in\right.$ $\left.\Phi_{+} \cap W \beta\right\} \subseteq R_{+}$. Since $R_{\beta, 0}=\overline{R_{\beta,+}} \cap R_{0}, R_{\beta, 0}$ is closed in $\operatorname{ray}(V)$.

Corollary. Assume that $W$ is irreducible and indefinite, $\alpha \in \overline{\mathscr{Z}} \backslash\{0\}$ and $\beta \in \Phi_{+}$.

(a) $\left.\operatorname{ri}(\mathscr{Z})=\operatorname{ri}\left(\operatorname{conv}\left(\cup E_{\alpha}\right)\right) \subseteq \operatorname{conv}\left(\cup E_{\alpha}\right)\right) \subseteq \overline{\mathscr{Z}}$.

(b) If $\alpha \in \operatorname{ri}(\mathscr{Z})$, then $\operatorname{conv}\left(\cup E_{\alpha}\right) \backslash\{0\}=\operatorname{ri}(\mathscr{Z})$ and $\langle\alpha, \alpha\rangle<0$.

(c) $\overline{\mathrm{R}_{\text {ext }}} \subseteq \overline{E_{\alpha}} \subseteq \bar{Z}$.

(d) $\overline{\mathrm{R}_{\text {ext }}} \subseteq R_{\beta, 0} \subseteq R_{0} \subseteq \bar{Z} \cap Q$.

Remarks. (1) If $W$ is irreducible affine, then, using the explicit description of $\mathscr{Z}$, (a)-(d) hold with the following change: in (b), $\langle\alpha, \alpha\rangle=0$.

(2) If $W$ is irreducible indefinite of rank at least three, then $E_{\alpha}$ is infinite by Lemma 7.7 and is contained in the compact set $\operatorname{ray}\left(\mathbb{R}_{\geq 0} \Pi\right)$, so $E_{\alpha}^{\prime} \neq \emptyset$. Let $0 \neq$ $\beta \in \overline{\mathscr{Z}}$ with $\mathbb{R}_{\geq 0} \beta \in E_{\alpha}^{\prime}$. Since $E_{\alpha}^{\prime}$ is closed and $W$-invariant, one has $\overline{E_{\beta}} \subseteq E_{\alpha}^{\prime}$. By applying (c) to $\beta$ instead of $\alpha$, one gets $\overline{\mathrm{R}_{\text {ext }}} \subseteq \overline{E_{\beta}} \subseteq E_{\alpha}^{\prime} \subseteq \bar{Z}$, which improves (c) under these extra assumptions.

(3) From [30, Example 2.16], one sees that, even for the standard root system of an irreducible finite rank Coxeter system, one may have $\mathrm{R}_{\text {ext }} \subsetneq R_{0}$.

(4) It will be shown in 21 that (for any irreducible $W$ ) $\overline{\mathrm{R}_{\text {ext }}}=R_{0}$. This leads to improvements in several results of this subsection and additional results not discussed here. It has been asked by Hohlweg and Ripoll whether (for irreducible W) $R_{0}=\bar{Z} \cap Q$.

Proof. In (a), the equality holds since $\operatorname{ri}(\mathscr{Z})=\operatorname{ri}(\overline{\mathscr{Z}})=\operatorname{ri}\left(\operatorname{cl}\left(\operatorname{conv}\left(\cup E_{\alpha}\right)\right)\right)=$ $\operatorname{ri}\left(\operatorname{conv}\left(\cup E_{\alpha}\right)\right)$, and the inclusions are trivial from the definitions. For (b), assume $\alpha \in \operatorname{ri}(\mathscr{Z})$. Then $\operatorname{ri}(\mathscr{Z}) \subseteq \operatorname{conv}\left(\cup\left(E_{\alpha}\right)\right) \backslash\{0\}$ by (a). On the other hand, since $\cup E_{\alpha}$ is contained in the salient cone $\mathbb{R}_{\geq 0} \Pi$ and

$$
\cup\left(E_{\alpha}\right) \backslash\{0\}=\left\{\lambda w(\alpha) \mid \lambda \in \mathbb{R}_{>0}, w \in W\right\} \subseteq \operatorname{ri}(\mathscr{Z}),
$$

one has $\operatorname{conv}\left(\cup\left(E_{\alpha}\right)\right) \backslash\{0\}=\operatorname{conv}\left(\cup\left(E_{\alpha}\right) \backslash\{0\}\right) \subseteq \operatorname{ri}(\mathscr{Z})$. If also $W$ is indefinite, then there is some $\alpha^{\prime} \in \mathscr{K}$ of the form $\alpha^{\prime}=\sum_{\gamma \in \Pi} c_{\gamma} \gamma$ with all $c_{\gamma}>0$ and $\left\langle\alpha^{\prime}, \Pi\right\rangle \subseteq \mathbb{R}_{<0}$. Then $\alpha^{\prime} \in \operatorname{ri}(\mathscr{K}) \subseteq \operatorname{ri}(\mathscr{Z})$ and $\left\langle\alpha^{\prime}, \alpha^{\prime}\right\rangle<0$. Using Lemma 5.9. one has $\langle\gamma, \gamma\rangle<0$ for all $\gamma \in \operatorname{conv}\left(\cup\left(E_{\alpha^{\prime}}\right) \backslash\{0\}\right)=\operatorname{ri}(\mathscr{Z})$ and so $\langle\alpha, \alpha\rangle<0$. This proves (b).

Since $\operatorname{conv}\left(\cup \overline{E_{\alpha}}\right)=\overline{\mathscr{Z}}$ by Proposition 7.5 , it follows from Lemma A.11 (b) that $\overline{E_{\alpha}} \supseteq \mathrm{R}_{\text {ext }}$. Taking closures gives $\bar{Z} \supseteq \overline{E_{\alpha}} \supseteq \overline{\mathrm{R}_{\text {ext }}}$, proving (c). In (d), $R_{\beta, 0} \subseteq R_{0} \subseteq$ $\bar{Z} \cap Q$ has already been noted. Observe that since $W \beta$ is infinite (by Proposition 1.18 (a)), it follows that $R_{\beta,+}$ has a limit point, which is necessarily in $R_{0}$ and so not in $R_{\beta,+}$. Hence there is some ray $\mathbb{R}_{\geq 0} \gamma \in R_{\beta, 0}$. Taking $\alpha=\gamma$ in (c), shows (in terms of the natural $W$-action on $\operatorname{ray}(V))$ that $\overline{\mathrm{R}_{\text {ext }}} \subseteq \overline{E_{\alpha}} \subseteq \overline{W R_{\beta, 0}}=R_{\beta, 0}$ and proves $(\mathrm{d})$.

7.10. We conclude this section with some miscellaneous properties of $\overline{\mathscr{Z}}$ related to its facial structure, about which much less is known than for $\mathscr{Z}$. These facts have interesting consequences which we do not go into here (but see $\$ 9$ for some applications to universal Coxeter groups).

Proposition. Let $\alpha \in \overline{\mathscr{Z}}$. Denote the minimal face of $\overline{\mathscr{Z}}$ containing $\alpha$ by $\overline{\mathscr{Z}}_{\alpha}$.

(a) $\overline{\mathscr{Z}} \cap \alpha^{\perp}$ is a face of $\overline{\mathscr{Z}}$. 
(b) If $\alpha$ is isotropic, then $\overline{\mathscr{Z}}_{\alpha}$ is a totally isotropic face of $\overline{\mathscr{Z}}$ and $\alpha \in \overline{\mathscr{Z}}_{\alpha} \subseteq$ $\overline{\mathscr{Z}} \cap \alpha^{\perp}$.

(c) If $\left(w_{n}\right)_{n \in \mathbb{N}}$ is any sequence in $W$, then $F:=\left\{\beta \in \overline{\mathscr{Z}} \mid \lim _{n \rightarrow \infty} w_{n} \beta=0\right\}$ is a totally isotropic face of $\overline{\mathscr{Z}}$.

(d) Let $W^{\prime}$ be an irreducible reflection subgroup such that $\left\langle\Pi_{W^{\prime}}, \alpha\right\rangle \subseteq \mathbb{R}_{\geq 0}$ but $\Pi_{W^{\prime}} \nsubseteq \alpha^{\perp}$. Then $W^{\prime}$ is finite.

Proof. In the terminology of Appendix A, $(\overline{\mathscr{Z}},-\overline{\mathscr{Z}})$ is semidual pair of stable cones with respect to $\langle-,-\rangle$ and $\overline{\mathscr{Z}} \cap \alpha^{\perp}$ is an exposed face of $\mathscr{Z}$ with respect to this semidual pair. In particular, (a) holds. Now assume that $\alpha$ is isotropic. It is trivial that $\alpha \in \overline{\mathscr{Z}}_{\alpha} \subseteq \overline{\mathscr{Z}} \cap \alpha^{\perp}$. Recall from the Appendix that

$$
\overline{\mathscr{Z}}_{\alpha}=\{x \in \overline{\mathscr{Z}} \mid t x+(1-t) y=\alpha \text { for some } y \in \overline{\mathscr{Z}} \text { and } 0<t<1\} \text {. }
$$

To show $\overline{\mathscr{Z}}_{\alpha}$ is totally isotropic, it suffices to show that $\langle x, x\rangle=0$ for all $x \in \overline{\mathscr{Z}}_{\alpha}$. Choose $y \in \overline{\mathscr{Z}}_{\alpha}$ and $0<t<1$ with $\alpha=t x+(1-t) y$. Since $\alpha$ is isotropic, Lemma 5.9 implies that $t x$ is isotropic and hence $x$ is isotropic as required for (b).

To prove (c), note first that $F$ is obviously a pointed cone contained in $\overline{\mathscr{Z}}$. To show $F$ is a face, suppose that $x, y \in \overline{\mathscr{Z}}$ with $x+y=\beta \in F$. Since $\left\langle\rho, w_{n} x\right\rangle+$ $\left\langle\rho, w_{n}(y)\right\rangle=\left\langle\rho, w_{n} \beta\right\rangle \rightarrow 0$ as $n \rightarrow \infty$ with $\left\langle\rho, w_{n} x\right\rangle,\left\langle\rho, w_{n}(y)\right\rangle \geq 0$, it follows that $\left\langle\rho, w_{n} x\right\rangle \rightarrow 0$ and $\left\langle\rho, w_{n}(y)\right\rangle \rightarrow 0$. By Lemma A.11, $w_{n} x \rightarrow 0$ and so $x \in F$. To see $F$ is totally isotropic, it suffices to show it contains no non-isotropic vector. This follows from Lemma 6.1(f).

Finally, we prove (d). Assume to the contrary that $W^{\prime}$ is infinite. Let $\Delta:=$ $\Pi_{W^{\prime}} \cap \alpha^{\perp} \subsetneq \Pi_{W^{\prime}}$. Lemma 1.10 gives $\operatorname{Stab}_{W^{\prime}}(\alpha)=W_{\Delta}$, since $\alpha \in \mathscr{C}_{W^{\prime}}$. Hence $\left|W^{\prime} \alpha\right|=\left|W^{\prime} / W_{\Delta}\right|$ is infinite by Lemma 1.20. By 4.4.1), $\left\{\langle\alpha-w \alpha, \rho\rangle \mid w \in W^{\prime}\right\}$ is not bounded above. But for any $w \in W^{\prime}, w \alpha \in \overline{\mathscr{Z}} \subseteq \mathbb{R}_{>0} \Pi$. Hence $\langle\alpha-w \alpha, \rho\rangle=$ $\langle\alpha, \rho\rangle-\langle w \alpha, \rho\rangle \leq\langle\alpha, \rho\rangle$, a contradiction which proves $(\mathrm{d})$.

7.11. This subsection formulates a result on limit points of $W$-orbits of ordered tuples of rays spanned by roots or in the imaginary cone. Other related results can be obtained by similar arguments.

Assume $\Phi$ is irreducible of indefinite type, $m \in \mathbb{N}_{\geq 1}$ and that $\alpha_{1}, \ldots, \alpha_{m} \in$ $\Phi \cup(\mathscr{Z} \backslash\{0\})$ are all non-isotropic (e.g. $\alpha_{i} \in \Phi \cup \operatorname{ri}(\mathscr{Z})$ for all $i$ ). Consider the tuple $\alpha:=\left(\mathbb{R}_{\geq 0} \alpha_{1}, \ldots, \mathbb{R}_{\geq 0} \alpha_{m}\right)$ of rays as a point of $(\operatorname{ray}(V))^{m}=\operatorname{ray}(V) \times \ldots \times \operatorname{ray}(V)$, which we equip with the product topology (which makes it compact) and diagonal $W$-action. The $W$-orbit of $\alpha$ in $(\operatorname{ray}(V))^{m}$ is infinite since the $W$-orbit of each ray $\mathbb{R}_{\geq 0} \alpha_{i}$ is infinite (by Proposition 1.18, Lemma 7.7 and an easy direct check if $W$ is dihedral). Let $\left(\beta_{1}, \ldots, \beta_{m}\right) \in V^{m}$ be such that $\beta:=\left(\mathbb{R}_{\geq 0} \beta_{1}, \ldots, \mathbb{R}_{\geq 0} \beta_{m}\right)$ is a limit point of the $W$-orbit $W \alpha$ in $(\operatorname{ray}(V))^{m}$. Replacing some $\alpha_{i} \in \Phi$ by their negatives and $\beta_{i}$ by suitable scalar multiples if necessary, we suppose without loss of generality by 5.35 .4 that $\beta_{i} \in \Phi_{+} \cup(\overline{\mathscr{Z}} \backslash\{0\})$ for all $i$. Note that $\beta_{i} \in \overline{\mathscr{Z}}$ if $\alpha_{i} \in \mathscr{Z}$, and the rays $\mathbb{R}_{\geq 0} \beta_{i}$ need not be pairwise distinct even if the $\mathbb{R}_{\geq 0} \alpha_{i}$ are.

Proposition. Let assumptions be as above. For some $i$ with $1 \leq i \leq m, \beta_{i}$ is isotropic. For any $i$ with $\beta_{i}$ isotropic, $\beta_{i} \in \overline{\mathscr{Z}} \cap \mathscr{Q}$ and $H:=\mathbb{R} \Pi \cap \beta_{i}^{\perp}$ is a supporting hyperplane of $\overline{\mathscr{Z}}$ in $\mathbb{R} \mathscr{Z}$ such that $H$ contains all of $\left\{\beta_{1}, \ldots, \beta_{m}\right\}$. In particular, there is a proper face of $\overline{\mathscr{Z}}$ containing all the $\beta_{j}$ which are isotropic.

Remarks. A more symmetric choice of supporting hyperplane with these properties would be $\mathbb{R} \Pi \cap \gamma^{\perp}$ where $\gamma \in \overline{\mathscr{Z}} \cap \mathscr{Q}$ is the sum of all $\beta_{i}$ which are isotropic. 
Proof. There is some sequence $\left(w_{n}\right)_{n \in \mathbb{N}}$ in $W$ such that the tuples $w_{n} \alpha$ for $n \in \mathbb{N}$ are pairwise distinct and $w_{n} \alpha \rightarrow \beta$ as $n \rightarrow \infty$. Passing to a subsequence of $\left(w_{n}\right)$ if necessary, we may suppose firstly that $w_{n} \alpha_{i} \in \mathbb{R}_{\geq 0} \Pi$ for all $i$ and secondly, by 4.4.1 and Lemma 4.3(d), that for some $i$ with $1 \leq i \leq m,\left\langle\rho, w_{n} \alpha_{i}\right\rangle \rightarrow \infty$ as $n \rightarrow \infty$. Now there is $\epsilon>0$ such that $\left\langle\rho, w_{n} \alpha_{j}\right\rangle>\epsilon$ for all $j=1, \ldots, m$ and all $n$ (for instance, using Lemma 1.21(b) for $j$ with $\alpha_{j} \in \Phi$ and Lemma 6.1(f) for other $j)$. Note that for any $j, k,\left\langle\rho, w_{n} \alpha_{j}\right\rangle^{-1} w_{n} \alpha_{j} \rightarrow\left\langle\rho, \beta_{j}\right\rangle^{-1} \beta_{j}$ in $V$ as $n \rightarrow \infty$ and hence, since $\left\langle w_{n} \alpha_{j}, w_{n} \alpha_{k}\right\rangle=\left\langle\alpha_{j}, \alpha_{k}\right\rangle$,

$$
\left\langle\rho, w_{n} \alpha_{j}\right\rangle^{-1}\left\langle\rho, w_{n} \alpha_{k}\right\rangle^{-1}\left\langle\alpha_{j}, \alpha_{k}\right\rangle \rightarrow\left\langle\rho, \beta_{j}\right\rangle^{-1}\left\langle\rho, \beta_{k}\right\rangle^{-1}\left\langle\beta_{j}, \beta_{k}\right\rangle
$$

in $\mathbb{R}$ as $n \rightarrow \infty$. Since the $\alpha_{j}$ are assumed non-isotropic, taking $k=j$ shows that $\beta_{j}$ is isotropic if and only if $\left\langle\rho, w_{n} \alpha_{j}\right\rangle \rightarrow \infty$ as $n \rightarrow \infty$ (in particular, there exists an isotropic $\beta_{i}$ from above). Also, for any isotropic $\beta_{j},\left\langle\beta_{j}, \beta_{k}\right\rangle=0$ for all $k$. Now fix $i$ such that $\beta_{i}$ is isotropic. Obviously $\beta_{i} \in \overline{\mathscr{Z}} \cap \mathscr{Q}$ since $\beta_{i} \notin \Phi_{+}$. By Lemma 5.9 $\left\langle\beta_{i}, \mathscr{Z}\right\rangle \subseteq \mathbb{R}_{\leq 0}$ and by Lemma 7.1. $\mathbb{R} \mathscr{Z}=\mathbb{R} \Pi \nsubseteq \beta_{i}^{\perp}$ (recall that $\Pi$ is irreducible of indefinite type). Hence $H:=\overline{\mathbb{R}} \cap \beta_{i}^{\perp}$ is a supporting hyperplane of $\overline{\mathscr{Z}}$ at $\beta_{i}$ as required.

7.12. For certain special irreducible $\Phi$ (for instance, $\Phi$ affine or $\Phi$ weakly hyperbolic as defined in $\$ 9$, the non-zero isotropic faces of $\bar{Z}$ are one-dimensional (they are rays). That this is not the case in general is shown by the following example.

Example. Consider the based root system $(\Phi, \Pi)$ in $(V,\langle-,-\rangle)$ in [30, Example 5.8]. It has linearly independent simple roots $\Pi=\{\alpha, \beta, \gamma, \delta, \epsilon\}$, where $\langle\alpha, \beta\rangle=$ $\langle\delta, \epsilon\rangle=-1,\langle\beta, \gamma\rangle=\langle\gamma, \delta\rangle=-1 / 2$ and all inner products of other distinct simple roots are 0 . Assume $V=\mathbb{R} \Pi$. The form $\langle-,-\rangle$ has signature $(3,1,1)$ and its radical is spanned by $\alpha+\beta-\gamma-\delta$. Note $z:=\alpha+\beta+\delta+\epsilon \in \mathscr{K} \subseteq \mathscr{Z}$ is isotropic. Let $J=\left\{s_{\alpha}, s_{\beta}\right\}, K:=\left\{s_{\delta}, s_{\epsilon}\right\}$ and $I:=J \cup K$ so $I, J, K \subseteq S$ and the components of $I$ are $J, K$, which are irreducible affine. Then since $z^{\perp}=\mathbb{R} \Pi_{I}$ and $I, J, K$ are facial, Theorem 5.1 gives

$$
\overline{\mathscr{Z}} \cap z^{\perp}=\overline{\mathscr{Z}} \cap \mathbb{R}_{I}=\overline{\mathscr{Z}}_{I}=\overline{\mathscr{Z}}_{J}+\overline{\mathscr{Z}}_{K}=\mathbb{R}_{\geq 0}(\alpha+\beta)+\mathbb{R}_{\geq 0}(\delta+\epsilon) .
$$

So $F:=\overline{\mathscr{Z}} \cap z^{\perp}=\mathbb{R}_{\geq 0}\{\alpha+\beta, \delta+\epsilon\} \subseteq \mathscr{K} \subseteq \mathscr{Z}$ is a two-dimensional totally isotropic face of $\overline{\mathscr{Z}}$. Clearly, $\mathbb{R} F$ is a maximal totally isotropic subspace of $(\mathbb{R} \Pi,\langle-,-\rangle)$. We claim that every ray in $F$ is a limit ray of rays spanned by positive roots.

To see this, it suffices to show that for any fixed $t \in \mathbb{R}$ with $0 \leq t \leq 1$, the ray $\mathbb{R}_{\geq 0}(t(\alpha+\beta)+(1-t)(\delta+\epsilon))$ is a limit ray of rays spanned by positive roots. For any $k, l \in \mathbb{N}$, set $\alpha_{2 k+1}:=\left(s_{\alpha} s_{\beta}\right)^{k}(\alpha):=(2 k+1) \alpha+2 k \beta \in \Phi_{+}$and $\epsilon_{2 l+1}:=$ $\left(s_{\epsilon} s_{\delta}\right)^{l}(\epsilon)=(2 l+1) \epsilon+2 l \delta \in \Phi_{+}$. Since $\left\langle\alpha_{2 k+1}, \epsilon_{2 l+1}\right\rangle=0$, one has

$$
\begin{aligned}
s_{\alpha_{2 k+1}} s_{\epsilon_{2 l+1}}(\gamma) & =\gamma-\left\langle\gamma, \alpha_{2 k+1}^{\vee}\right\rangle \alpha_{2 k+1}-\left\langle\gamma, \epsilon_{2 l+1}^{\vee}\right\rangle \epsilon_{2 l+1}=\gamma+2 k \alpha_{2 k+1}+2 l \epsilon_{2 l+1} \\
& =\gamma+2 k(2 k+1) \alpha+4 k^{2} \beta+4 l^{2} \delta+2 l(2 l+1) \epsilon \in \Phi_{+} .
\end{aligned}
$$

One may choose sequences $\left(k_{n}\right),\left(l_{n}\right)$ in $\mathbb{N}$ with $k_{n}, l_{n} \rightarrow \infty$ and $\frac{k_{n}^{2}}{k_{n}^{2}+l_{n}^{2}} \rightarrow t$ as $n \rightarrow \infty$. It is easy to see from above that as $n \rightarrow \infty$,

$$
\frac{1}{4\left(k_{n}^{2}+l_{n}^{2}\right)} s_{\alpha_{2 k_{n}+1}} s_{\epsilon_{2 l_{n}+1}}(\gamma) \rightarrow t(\alpha+\beta)+(1-t)(\delta+\epsilon)
$$

from which the claim follows readily.

Now define the quotient space $\left(V^{\prime},\langle-,-\rangle^{\prime}\right)$ of $(V,\langle-,-\rangle)$ by its radical, where $V^{\prime}:=V / \mathbb{R}(\alpha+\beta-\gamma-\delta)$. Let $L: V \rightarrow V^{\prime}$ be the natural map. Then $(\Phi, \Pi)$ on 
MATTHEW J. DYER

$(V,\langle-,-\rangle)$ is a canonical lift of a based root system $\left(\Phi^{\prime}, \Pi^{\prime}\right):=(L(\Phi), L(\Pi))$ for $\left(V^{\prime},\langle-,-\rangle^{\prime}\right)$, with $L$ as the associated canonical map (see 1.4 but note that the roles of $\Phi$ and $\Phi^{\prime}$ are swapped here in relation to the notation there). From Proposition $5.10 L$ induces a surjective map $R_{W, 0} \rightarrow R_{W^{\prime}, 0}$ on corresponding sets of limit rays of positive roots. This induced map sends all limit rays $\mathbb{R}_{\geq 0}(t(\alpha+\beta)+(1-t)(\gamma+\delta))$ with $0 \leq t \leq 1$ to the same limit ray $\mathbb{R}_{\geq 0}(L(\alpha+\beta))$ and in particular the induced map is not bijective. Hence the set of limit rays of positive roots can depend not only on the Coxeter group $W$ but also on the chosen root system for it, even for irreducible $W$.

\section{Supports}

8.1. We say $\Delta$ is a support of $v \in \mathbb{R}_{\geq 0} \Pi$ (with respect to $\Pi$ ) if $\Delta \subseteq \Pi$ and there is an expression $v=\sum_{\alpha \in \Delta} c_{\alpha} \alpha$ where all $c_{\alpha}>0$. The only support of 0 is $\emptyset$, but if $\Pi$ is linearly dependent, an arbitrary element $v \in \mathbb{R}_{\geq 0} \Pi$ may have many supports. Note that for any $\Delta \subseteq \Pi, \Delta$ is a set of representatives for the extreme rays of the polyhedral cone $\mathbb{R}_{\geq 0} \Delta$, which has as its relative interior the set of elements of $\mathbb{R}_{\geq 0} \Pi$ with $\Delta$ as a support.

Define $\mathscr{K}^{\mathrm{c}}$ to be the subset of $\mathscr{K}$ of all non-zero elements with some connected support, and $\mathscr{Z}^{\mathrm{c}}:=\cup_{w \in W} w \mathscr{K}^{\mathrm{c}}$.

Lemma. Let $\alpha, \gamma \in \mathbb{R}_{\geq 0} \Pi$.

(a) The set of supports of $\alpha$ is closed under finite unions.

(b) If $\alpha, \gamma$ have connected supports $\Delta, \Delta^{\prime}$ and $\Delta, \Delta^{\prime}$ are not separated (e.g. $\langle\alpha, \gamma\rangle \neq 0)$, then for any $c, d \in \mathbb{R}_{>0}, c \alpha+d \gamma$ has a connected support $\Delta \cup \Delta^{\prime}$.

(c) If $\alpha$ has supports $\Delta$ and $\Delta^{\prime}$, and $\Delta$ and $\Delta^{\prime}$ are separated, then $\alpha$ is isotropic.

(d) If $\Delta$ and $\Delta^{\prime}$ are connected supports of $v$ and $v$ is non-isotropic, then $\Delta \cup \Delta^{\prime}$ is a connected support of $v$.

(e) If $\alpha$ is non-isotropic with some connected support, then the set of its connected supports has an inclusion-maximal element.

(f) Let $\Delta \subseteq \Pi$ and $\Delta^{\prime}:=\mathbb{R} \Delta \cap \Pi$. Then each $\beta \in \Delta^{\prime} \backslash \Delta$ is joined to some vertex of $\Delta$. The number of connected components of $\Delta^{\prime}$ is no more than the number of connected components of $\Delta$; in particular, if $\Delta$ is connected, then $\Delta^{\prime}$ is connected.

(g) If $\Delta$ is a support of $\alpha$, and $\Delta \subseteq \Delta^{\prime} \subseteq \mathbb{R} \Delta \cap \Pi$, then $\Delta^{\prime}$ is a support of $\alpha$.

(h) Any $\beta \in \Phi_{+}$has a connected support.

(i) Any element of $\mathscr{Z}^{\mathrm{c}}$ has a connected support. More precisely, if $\Delta$ is a connected support of $k \in \mathscr{K}^{\mathrm{c}}$, then for any $w \in W$, wk has a connected support $\Delta^{\prime}$ containing $\Delta$.

(j) Let $W^{\prime}$ be a finitely generated reflection subgroup of $W$, and $\beta \in \mathbb{R}_{\geq 0} \Pi_{W^{\prime}}$ have a connected support with respect to $\Pi_{W^{\prime}}$. Then $\beta \in \mathbb{R}_{\geq 0} \Pi$ and $\beta$ has a connected support with respect to $\Pi$.

(k) Any element of $\mathscr{K}$ (resp., $\mathscr{Z}$ ) can be written as a sum of finitely many pairwise orthogonal elements of $\mathscr{K}^{\mathrm{c}}$ (resp., $\mathscr{Z} \mathrm{c}$ ).

Remarks. (1) Except for (e) and (g), which require just finiteness of $\Pi$, the above do not require the assumptions 4.1(i)-(iii).

(2) One might study along similar lines the properties of the support polytopes $\left\{\left(c_{\alpha}\right)_{\alpha \in \Pi} \mid\right.$ all $\left.c_{\alpha} \in \mathbb{R}_{\geq 0}, v=\sum_{\alpha} c_{\alpha} \alpha\right\}$ of elements $v \in \mathbb{R}_{\geq 0} \Pi$. 
Proof. We omit the simple proofs of (a)-(f). For (g), note that $\alpha$ is in the relative interior of $\mathbb{R}_{\geq 0} \Delta$ and hence in that of $\mathbb{R}_{\geq 0} \Delta^{\prime}$. We prove (h) by induction on $l\left(s_{\beta}\right)$. If $l(\beta)=1$, then $\{\beta\}$ is a connected support of $\beta$. Otherwise, there is some $\gamma \in \Pi$ with $l\left(s_{\gamma} s_{\beta} s_{\gamma}\right)=l\left(s_{\beta}\right)-2$. Set $\delta:=s_{\gamma}(\beta) \in \Phi_{+}$, which has connected support by induction and satisfies $c:=-\left\langle\delta, \gamma^{\vee}\right\rangle>0$ by [3] Lemma 3.4]. By (c), $\beta=s_{\gamma}(\delta)=\delta+c \gamma$ has a connected support.

For (i), let $k \in \mathscr{K}^{\mathrm{c}}$ with connected support $\Delta$. We show $z \in W k$ has a connected support $\Delta^{\prime}$ containing $\Delta$ by induction on the minimum value $n$ of $l(w)$ such that $z=w k$ with $w \in W$. If $n=0$, the result is trivial. Otherwise, write $w=s_{\alpha_{n}} \ldots s_{\alpha_{1}}$ with $\alpha \in \Pi$ and $n \geq 1$ minimal. By induction, $z^{\prime}:=s_{\alpha_{n-1}} \ldots s_{\alpha_{1}} k$ has a connected support $\Delta^{\prime} \supseteq \Delta$. We claim that

$$
c:=\left\langle\alpha_{n}^{\vee}, z^{\prime}\right\rangle=\left\langle s_{\alpha_{1}} \cdots s_{\alpha_{n-1}}\left(\alpha_{n}^{\vee}\right), k\right\rangle<0 .
$$

For if $c>0$, then $s_{\alpha_{1}} \cdots s_{\alpha_{n-1}}\left(\alpha_{n}\right) \in \Phi_{-}$since $k \in-\mathscr{C}$, and $s_{\alpha_{n}} \ldots s_{\alpha_{1}}$ couldn't be reduced by Lemma 1.13 (b); if $c=0$, then $w k=s_{\alpha_{n-1}} \cdots s_{\alpha_{1}} k$ contrary to minimality of $n$. Hence $w k=z^{\prime}-c \alpha_{n}$ has a connected support $\Delta^{\prime} \cup\left\{\alpha_{n}\right\}$ by (c) since $-c>0$. This proves (i).

Next, we prove $(\mathrm{j})$. Write $\beta=\sum_{\gamma \in \Delta} c_{\gamma} \gamma$ where $\Delta \subseteq \Pi_{W^{\prime}}$ is connected and each $c_{\gamma}>0$. Also write $\Delta=\left\{\delta_{1}, \ldots, \delta_{n}\right\}$ where for each $i$ with $1 \leq i \leq n$, there is some $j<i$ with $\left\langle\delta_{j}, \delta_{i}\right\rangle \neq 0$. By (h), each $\delta_{i}$ has some connected support $\Delta_{i}$. By repeated application of (c), it follows that $\Delta_{i}^{\prime}:=\Delta_{1} \cup \cdots \cup \Delta_{i}$ is connected for $i=1,2, \ldots, n$. But $\Delta_{n}^{\prime}$ is clearly a support for $\beta$, and (j) follows.

To prove (k), write $\alpha \in \mathscr{K}$ as $\alpha=\sum_{\beta \in \Delta} c_{\beta} \beta$ for some $\Delta \subseteq \Pi$ where all $c_{\beta}>0$. Let $\Delta_{1}, \ldots, \Delta_{m}$ be the components of $\Delta$. Let $\alpha_{i}:=\sum_{\beta \in \Delta_{i}} c_{\beta} \beta \in \mathscr{K}$, so $\alpha=\alpha_{1}+\ldots+\alpha_{m}$ and $\left\langle\alpha_{i}, \alpha_{j}\right\rangle=0$ if $i \neq j$. Then $\left\langle\alpha_{i}, \beta\right\rangle=\langle\alpha, \beta\rangle \leq 0$ for all $\beta \in \Delta_{i}$ and $\left\langle\alpha_{i}, \Pi \backslash \Delta_{i}\right\rangle \subseteq \mathbb{R}_{\leq 0}$ so $\alpha_{i} \in \mathscr{K}^{\mathrm{c}}$. Now if $\gamma \in \mathscr{Z}$, choose $w \in W$ so $\alpha:=w^{-1} \gamma \in \mathscr{K}$. Write $\alpha=\alpha_{1}+\ldots+\alpha_{m}$ as above. Then $\gamma=\gamma_{1}+\ldots+\gamma_{m}$ where $\gamma_{i}:=w \alpha_{i} \in \mathscr{Z}^{\mathrm{c}}$ are pairwise orthogonal as required.

8.2. By Lemma 8.1(a), any $\alpha \in \mathbb{R}_{\geq 0} \Pi$ has a maximum (under inclusion) support $\Delta$. It is the set of representatives (in $\Pi$ ) of the extreme rays of the inclusion minimal element of the set of faces of the polyhedral cone $\mathbb{R}_{\geq 0} \Pi$ containing $\alpha$. We call $\Delta$ the facial support of $\alpha$.

The following simple examples illustrate features of the above notion of supports if $\Pi$ is not linearly independent.

Example. Suppose that $\Pi=\{\alpha, \beta, \gamma, \delta\}$ has irreducible affine components $\{\alpha, \beta\}$ and $\{\gamma, \delta\}$ and that the space of linear relations amongst elements of $\Pi$ is spanned over $\mathbb{R}$ by the single relation $\alpha+\beta-\gamma-\delta=0$.

The non-isotropic element (root) $v=s_{\alpha}(\beta)=2 \alpha+\beta$ has a connected support $\{\alpha, \beta\}$, a disconnected support $\{\alpha, \gamma, \delta\}$ (since $v=\alpha+\gamma+\delta$ ) and disconnected facial support $\Pi$ (since $v=\frac{3}{2} \alpha+\frac{1}{2} \beta+\frac{1}{2} \gamma+\frac{1}{2} \delta$ ).

The isotropic element $v^{\prime}=\alpha+\beta=\gamma+\delta$ spans the isotropic ray of both $\{\alpha, \beta\}$ and $\{\gamma, \delta\}$, which are its connected supports (and are separated from one another), and it has disconnected facial support $\Pi$.

8.3. In this subsection, we do not use or assume the conditions 4.1(i)-(iii). More generally than in the above example, let $\Phi$ be any root system with positive roots $\Phi_{+}$and simple roots $\Pi$ in $V$ as in 1.3 . Suppose that $U$ is a subspace of the radical of $\langle-,-\rangle$ such that no non-zero non-negative combination of simple roots is in 
$U$. Then there is an induced bilinear form on $V / U$ and the image of $\Phi$ in the quotient $V / U$ is a root system with simple roots given by the image $\Pi^{\prime}$ of $\Pi$ in $V / U$, and with Coxeter system $\left(W^{\prime}, S^{\prime}\right)$ naturally identified with that associated with $\Pi$. The canonical epimorphism $p: V \rightarrow V / U$ induces a bijection of corresponding root systems, positive root systems and systems of simple roots (cf. [34, 6.1]). We remark that any root system (regarded as a subset of its linear span, with the restriction of the ambient bilinear form) arises in this way by this process of dividing out a suitable subspace of the radical of the ambient vector space of some root system with linearly independent simple roots (see 1.4). We also remark that this construction provides a "canonical support" of any positive root $\alpha$ in $V / U$, namely the image under $p$ of the support of the unique positive root $\tilde{\alpha} \in V$ with $p(\tilde{\alpha})=\alpha$. The canonical support need not coincide with the facial support; for example, in Example 8.2 $v$ has canonical support $\{\alpha, \beta\}$.

In any case, one may construct similar examples to Example 8.2 involving $W$ with infinite type components by taking suitable $\Pi$ and subspaces of the radical.

8.4. Return to the standing assumptions 4.1(i)-(iii). The next result concerns supports of elements of $\mathscr{K}$.

Lemma. Let $0 \neq v \in \mathscr{K}$. Consider any expression $v=\sum_{\alpha \in \Delta} c_{\alpha} \alpha$ with $\Delta \subseteq \Pi$ and with all $c_{\alpha}>0$ (e.g. $\Delta$ could be the facial support of $v$ ). Let $\Delta_{1}, \ldots, \Delta_{n}$ denote the irreducible components of $\Delta$ and set $v_{i}:=\sum_{\alpha \in \Delta_{i}} c_{\alpha} \alpha$.

(a) We have $v=v_{1}+\ldots+v_{n}$ where $\left\langle v_{i}, v_{j}\right\rangle=0$ if $i \neq j$ and each $v_{i} \in \mathscr{K}^{\mathrm{c}}$.

(b) Either $\Delta_{i}$ is of indefinite type (in which case $\left\langle v, v_{i}\right\rangle=\left\langle v_{i}, v_{i}\right\rangle<0$ and there is some $\alpha \in \Delta_{i}$ with $\left\langle v_{i}, \alpha\right\rangle<0$ ) or of affine type (in which case $\left\langle v, v_{i}\right\rangle=\left\langle v_{i}, v_{i}\right\rangle=0$ and $v_{i}$ spans the isotropic ray of $\left.\Delta_{i}\right)$.

(c) $v$ is isotropic if and only if all $\Delta_{i}$ are of affine type.

(d) (cf. [33, Exercise 5.9]) For each $i$ for which $\Delta_{i}$ is of indefinite type, the connected components of $\left\{\alpha \in \Delta_{i} \mid\left\langle v_{i}, \alpha\right\rangle=0\right\}$ are all of finite type.

(e) If $v$ is isotropic, then $v$ has a connected support if and only if $v$ spans the isotropic ray of some irreducible (necessarily affine) component of the facial support of $v$. In that case, any connected support of $v$ is a connected component of the facial support of $v$.

(f) If $v$ is non-isotropic then it has a connected support if and only if there is a unique component $\Gamma$ of the facial support of $v$ which is of indefinite type. Then any connected support of $v$ is contained in $\Gamma$.

Proof. Part (a) is from the proof of 8.1(a). We prove (b). We have $\langle v, \alpha\rangle=$ $\left\langle v_{i}, \alpha\right\rangle \leq 0$ for all $\alpha \in \Delta_{i}$. Since $\Delta_{i}$ is connected, it is of either affine or indefinite type; it is of affine type if and only if $\left\langle v_{i}, \alpha\right\rangle=0$ for all $\alpha \in \Delta$, in which case $v_{i}$ is a representative of the isotropic ray of $\Delta_{i}$. We have

$$
\left\langle v_{i}, v_{i}\right\rangle=\sum_{\alpha \in \Delta_{i}} c_{\alpha}\left\langle v_{i}, \alpha\right\rangle \leq 0
$$

with equality if and only if $\Delta_{i}$ is of affine type. This proves (b).

By (a) and (b), $\langle v, v\rangle=\sum_{i}\left\langle v_{i}, v_{i}\right\rangle \leq 0$, with equality if and only if all $\Delta_{i}$ are affine, proving (c).

Part (d) may be deduced from Proposition 7.10(d). We provide instead the following alternative argument. Let $\Gamma$ be a connected component of $\left\{\alpha \in \Delta_{i} \mid\right.$ 
$\left.\left\langle v_{i}, \alpha\right\rangle=0\right\}$. By (b), we have $\Gamma \subsetneq \Delta_{i}$. Let $v_{\Gamma}:=\sum_{\gamma \in \Gamma} c_{\gamma} \gamma$. Then for $\alpha \in \Gamma$, $\left\langle v_{i}, \alpha\right\rangle=0$ so

$$
\left\langle v_{\Gamma}, \alpha\right\rangle=\sum_{\gamma \in \Gamma} c_{\gamma}\langle\gamma, \alpha\rangle=-\sum_{\gamma \in \Delta_{i} \backslash \Gamma} c_{\gamma}\langle\gamma, \alpha\rangle \geq 0 .
$$

Moreover, since $\Delta_{i}$ is connected, there is some $\alpha \in \Gamma$ which is joined to some $\gamma \in \Delta_{i} \backslash \Gamma$, and then $\left\langle v_{\Gamma}, \alpha\right\rangle>0$. By the classification [33] of matrices (see 4.5), it follows that $\Gamma$ is of finite type.

For the proofs of (e)-(f), choose the expression for $v$ so $\Delta$ is the facial support of $v$. We may apply (a)-(c) above to both the facial support $\Delta$ of $v$, and also some arbitrary (possibly connected) support $\Delta^{\prime}$ of $v$. Necessarily, we have $\Delta^{\prime} \subseteq \Delta$. Each irreducible component $\Delta_{j}^{\prime}$ of $\Delta^{\prime}$, for $j=1, \ldots, m$, is contained in some irreducible component $\Delta_{i_{j}}$ of $\Delta$. Write $v=\sum_{j} v_{j}^{\prime}$ where $v_{j}^{\prime}$ has support $\Delta_{j}^{\prime}$.

For (e), suppose $v$ is isotropic. Then each $\Delta_{j}^{\prime}=\Delta_{i_{j}}$, since $\Delta_{j}^{\prime} \subseteq \Delta_{i_{j}}$ are both irreducible affine. In particular, if $\Delta^{\prime}$ is connected, then $\Delta^{\prime}=\Delta_{1}^{\prime}=\Delta_{i_{1}}$ and by (b)-(c), $v$ spans the isotropic ray of $\Delta^{\prime}=\Delta_{i_{1}}$. Conversely, if $v$ spans the isotropic ray of $\Delta_{i}$, then $\Delta_{i}$ is a connected support of $v$. This proves (e).

Now for (f), suppose that $v$ is non-isotropic. If $v_{i}$ is non-isotropic, then $i_{j}=i$ for some $i$, since otherwise $\left\langle v_{i}, v_{i}\right\rangle=\left\langle v, v_{i}\right\rangle=\sum_{j}\left\langle v_{j}^{\prime}, v_{i}\right\rangle=0$. It follows that the number of components $\Delta_{j}^{\prime}$ of $\Delta^{\prime}$ is at least equal to the number of non-affine components $\Delta_{i}$ of $\Delta$. If we take $\Delta^{\prime}=\Delta_{1}^{\prime}$ to be any connected support of $v$, it cannot be of affine type by (b). It follows that there is exactly one indefinite component $\Delta_{i_{1}}$ of $\Delta$, and $\Delta^{\prime} \subseteq \Delta_{i_{1}}$, proving (f).

8.5. Here we supplement the information on facial subsets of $\Pi$ given by Lemma 2.10 by additional facts under the assumptions 4.1(i)-(iii).

Lemma. (a) (49, Theorem 4, 1)], [34, 2.2.4]). Any subset $I$ of $S$ such that all irreducible components of $\Pi_{I}$ are of finite type is facial.

(b) ([49, Theorem 4, 2)]). Let $I$ be a subset of $S$ such that $I^{\perp}=\emptyset$ and every component of $\Pi_{I}$ is of affine type. Then $I$ is facial.

(c) Let I be a facial subset of $S$. Let $M$ be a subset of $I$ such that $\Pi_{M}$ is a union of irreducible components of $\Pi_{I}$ and $\Pi_{M}$ contains all the irreducible affine components of $\Pi_{I}$. Then $M$ is a facial subset of $S$.

(d) Let $I \subseteq S$. Then the facial closure of $W_{I}$ coincides with the standard facial closure of $W_{I}$.

Proof. Note that $\emptyset=\Pi \cap \rho^{\perp}$ is facial in $\Pi$, so $\emptyset$ is facial in $S$ and (a) follows from 2.10 (b). In (b), let $I_{1}, \ldots, I_{p}$ be the irreducible components of $I$ (all of affine type), and write $\mathscr{K}_{I_{i}}=\mathbb{R}_{\geq 0} \delta_{i}$. Then $z:=-\left(\delta_{1}+\cdots+\delta_{p}\right) \in-\mathscr{K}_{I} \subseteq-\mathscr{K} \subseteq \mathscr{C}$ and $z^{\perp} \cap \Pi=\Pi_{I}$ since $z \perp \Pi_{I}, \Pi_{I}$ is a support of $-z$ and $I^{\perp}=\emptyset$. This proves (b).

Now we prove (c) (compare Proposition 2.13. Let $v_{J}=\sum_{\alpha \in \Phi_{J,+}} \alpha$ where $J \subseteq I$ is such that $\Pi_{J}$ is the union of all finite type irreducible components of $\Pi_{I}$ which are not in $\Pi_{M}$. It is well known that $\left\langle v_{J}, \alpha\right\rangle=1>0$ for all $\alpha \in \Pi_{J}$ (this is easily seen since $s_{\alpha}\left(v_{J}\right)=v_{J}-2 \alpha$, using that $s_{\alpha}$ permutes $\left.\Phi_{J,+} \backslash\{\alpha\}\right)$. Let $L \subseteq I$ be such that $\Pi_{L}$ is the union of all indefinite type components of $\Pi_{I}$ which are not in $\Pi_{M}$. Since each component of $\Pi_{L}$ is indefinite, there is by 4.5 some element $v_{L} \in V$ expressible as a strictly negative real linear combination of elements of $\Pi_{L}$ such that $\left\langle v_{L}, \alpha\right\rangle>0$ for all $\alpha \in \Pi_{L}$. We have

$$
\left\langle v_{L}, \Pi_{L}\right\rangle \subseteq \mathbb{R}_{>0}, \quad\left\langle v_{L}, \Pi_{I \backslash L}\right\rangle \subseteq\{0\}, \quad\left\langle v_{J}, \Pi_{J}\right\rangle \subseteq \mathbb{R}_{>0}, \quad\left\langle v_{J}, \Pi_{I \backslash J}\right\rangle \subseteq\{0\} .
$$


Hence $v_{J}+v_{L} \in \mathscr{C}_{I}$ and $\Pi_{I} \cap\left(v_{J}+v_{L}\right)^{\perp}=\Pi_{M}$. By definition, $M$ is a facial subset of $I$ (i.e. it is facial for $\left(W_{I}, I\right)$ with simple roots $\left.\Pi_{I}\right)$ and by 4.2 (f), $M$ is facial in $S$ as required.

Finally, we prove (d). For any $I \subseteq S$, let $I_{\infty}$ (resp., $I_{0}$ ) denote the union of all infinite type (resp., finite type) components of $I$, so $I=I_{\infty} \cup I_{0}$ with $I_{0}$ and $I_{\infty}$ separated. Assume first that $I$ is special i.e. $I=I_{\infty}$. Write the facial closure of $W_{I}$ as $d W_{K} d^{-1}$ where $K \subseteq S$ is facial and, without loss of generality, $d \in W^{K}$. Then $\Pi_{I} \subseteq d \Pi_{K}$ and hence $d^{-1} \Pi_{I} \subseteq \Pi_{K}$. By Remark 1.15 , it follows that $d^{-1} \in W_{I^{\perp}}$ and $\Pi_{I}=d^{-1} \Pi_{I} \subseteq \Pi_{K}$. Hence $W_{K}$ is a facial subgroup of minimal rank containing $W_{I}$ (since $W_{K} \supseteq W_{I}$ has the same rank as the facial closure $d W_{K} d^{-1}$ of $W_{I}$ ) and it is therefore the facial closure of $W_{I}$. Finally, $W_{K}$ is the standard facial closure of $W_{I}$ since $W_{K}$ is standard facial and the standard facial closure of any subset of $W$ contains the facial closure of that subset.

Now consider the case of general $I \subseteq S$. We proceed by downward induction on $|I|$, the assertion to be proved being trivial if $I=S$. Suppose now that $I \subsetneq S$ and denote its facial closure as $W^{\prime}$. Let $J \subseteq S$, where $J \supseteq I_{\infty}$, be such that $W_{J}$ is the standard facial closure of $W_{I_{\infty}}$. Note that $J$ is special, for $I_{\infty} \subseteq J_{\infty}$, where $J_{\infty}$ is a facial subset of $S$ by (c), and so $J=J_{\infty}$. From the special case $I=I_{\infty}$ already treated, $W_{J}$ is the facial closure of $W_{I_{\infty}}$, so $W^{\prime} \supseteq W_{J}$ and $W^{\prime}$ is the facial closure of $W_{J \cup I_{0}}$. Also, the standard facial closure of $W_{I}$ coincides with that of $W_{J \cup I_{0}}$ If $I \subsetneq J \cup I_{0}$, then by induction, the standard facial closure $W_{K}$ of $W_{J \cup I_{0}}$ (and $W_{I}$ ) is equal to $W^{\prime}$ by induction. If $I=J \cup I_{0}$, then $I_{\infty} \subseteq J \subseteq I_{\infty}, I_{\infty}=J$ is facial and hence $I=J \cup I_{0}$ is facial by Lemma 2.9 (c). In either case, we have the desired conclusion.

8.6. We next describe the relation of the imaginary cone to the facial subsets of $\Pi$ in the most general affine case.

In this subsection only, let $\Pi_{i}$ for $i \in I$ be the irreducible components of $\Pi$, and assume that all $\Pi_{i}$ are of affine type. Each set $\Pi_{i}$ is linearly independent. Let $W_{i}$ be the affine Weyl group corresponding to $\Pi_{i}$. Write $\mathscr{K}_{i}=\mathbb{R}_{\geq 0} \delta_{i}$ where $\delta_{i} \in \mathbb{R}_{\geq 0} \Pi_{i} \subseteq \mathbb{R}_{\geq 0} \Pi$ spans the isotropic ray of $\Pi_{i}$. We have $\mathscr{Z}_{i}:=W_{i} \mathscr{K}_{i}=\mathscr{K}_{i}$ and by 3.2 (d) that $\mathscr{Z}=\sum_{i} \mathscr{Z}_{i}=\sum_{i} \mathscr{K}_{i}=\mathscr{K}$ which is a polyhedral cone. Note that some of the $\delta_{i}$ may lie in the relative interior of faces of $\mathscr{K}$ of dimension greater than 1 or may span the same extreme ray of $\mathscr{K}$.

Lemma. (a) Let $P$ be a face of the cone $\mathscr{Z}$, and $J=\left\{i \in I \mid \delta_{i} \in P\right\}$. For $i \in I$, let $\Delta_{i} \subseteq \Pi_{i}$ be such that $\Delta_{i}:=\Pi_{i}$ if $i \in J$, and $\Delta_{i}$ is a proper subset of $\Pi_{i}$ otherwise. Then $\Delta:=\cup_{i \in I} \Delta_{i}$ is a facial subset of $\Pi$.

(b) Each facial subset $\Delta$ of $\Pi$ arises as in (a) from a unique choice of face $P$ of $\mathscr{Z}$ and sets $\Delta_{i} \subseteq \Pi_{i}$ satisfying the conditions of (a).

Remarks. (1) It is easy to see that (up to linear isomorphism), any pair $(C, U)$ of a polyhedral cone $C$ in a vector space $U$ with $C=\mathbb{R} U$ is isomorphic to a pair $(\mathscr{Z}, \mathbb{R} \mathscr{Z})$ arising as above from some affine root system $\Phi$. Moreover, given any multiset $R$ of rays in $C$ including all the extreme rays of $C$ with multiplicity at least one, one could choose $\Phi$ so in addition, $R$ corresponds under the isomorphism to the multiset of isotropic rays of the irreducible components of $\Phi$

(2) One obtains from Lemmas 8.6 and 2.13 a complete description of all the facial subsets of $\Pi$ when $\Pi$ has only finite type and affine type components. In this case, one easily sees that the faces of $\mathscr{Z}$ are in natural bijection with the special 
facial subgroups $W^{\prime}$ of $W$, via $W^{\prime} \mapsto \mathscr{Z}_{W^{\prime}}$. We shall show this holds in general in Section 10.

Proof. We first prove (b). Choose $\phi \in \mathscr{C}$ and consider the corresponding facial subset $\Delta:=\Pi \cap \phi^{\perp}$ of $\Pi$. We have $\langle\phi, \mathscr{Z}\rangle \subseteq \mathbb{R}_{\geq 0}$. Let $P:=\mathscr{Z} \cap \phi^{\perp}$, which is a face of $\mathscr{Z}$. Let $J:=\left\{i \in I \mid \delta_{i} \in P\right\}$ and $\Delta_{i}:=\Delta \cap \Pi_{i}$. Note that $\delta_{i}$ is a strictly positive linear combination of $\Pi_{i}$ and $\left\langle\phi, \Pi_{i}\right\rangle \subseteq \mathbb{R}_{>0}$. If $i \in J$, then $\left\langle\phi, \delta_{i}\right\rangle=0$, so it follows $\left\langle\phi, \Pi_{i}\right\rangle=0, \Pi_{i} \subseteq \Delta$ and therefore $\Delta_{i}=\Pi_{i}$ in this case. On the other hand, if $i \notin J$, then $\left\langle\phi, \delta_{i}\right\rangle>0$ so $\Delta_{i} \subsetneq \Pi_{i}$. Hence $\Delta$ arises as in (a). Clearly, $\Delta_{i}=\Pi_{i} \cap \Delta, J=\left\{i \in I \mid \Delta_{i}=\Pi_{i}\right\}$, and $P=\sum_{j \in J} \mathbb{R}_{\geq 0} \delta_{j}$ are uniquely determined by $\Delta$.

Conversely, let $P, J, \Delta_{i}$ be as in (a). Choose $\Gamma_{i} \subseteq \Pi_{i}$ as follows. If $i \in J$, let $\Gamma_{i}=\Pi_{i}^{\prime}$ where $\Pi_{i}^{\prime} \subseteq \Pi_{i}$ is the set of simple roots of the finite Weyl group corresponding to $\Phi_{i}$ (in fact, we need only $\Pi_{i}^{\prime} \subseteq \Pi_{i}$ and $\left|\Pi_{i} \backslash \Pi_{i}^{\prime}\right|=1$ ) . If $i \in I \backslash J$, let $\Gamma_{i}$ be any subset of $\Pi_{i}$ such that $\left|\Pi_{i} \backslash \Gamma_{i}\right|=1$ and $\Delta_{i} \subseteq \Gamma_{i}$. Note that $\Gamma:=\cup_{i} \Gamma_{i}$ has all components of finite type; hence it is facial and linearly independent and the restriction of $\langle-,-\rangle$ to $\mathbb{R} \Gamma$ is positive definite by Lemmas 8.5(a) and 2.9(c). It follows that $\mathbb{R} \Pi=\mathbb{R} \Gamma \oplus \mathbb{R} \mathscr{Z}$, an orthogonal direct sum with $\mathbb{R} \mathscr{Z}$ as radical, where $\mathbb{R} \Pi$ is positive semidefinite. Choose, by non-degeneracy of $\langle-,-\rangle$, a subspace $U^{\prime} \supseteq \mathbb{R} \Pi$ of $V$ such that the restriction of $\langle-,-\rangle$ to $U^{\prime}$ is non-singular and such that the codimension of $\mathbb{R} \Pi$ in $U^{\prime}$ is equal to the dimension $N$ of $\mathbb{R} \mathscr{Z}$. Write $(\mathbb{R} \Gamma)^{\perp} \cap U^{\prime}=\mathbb{R} \mathscr{Z}+U$ where $U$ is a totally isotropic subspace of $U^{\prime}$ of dimension $N$. Then the restriction of $\langle-,-\rangle$ to $\mathbb{R} \mathscr{Z}+U$ is non-singular of signature $(N, N, 0)$ (it is a direct sum of $N$ hyperbolic planes) and the induced bilinear form $\mathbb{R} \mathscr{Z} \times U \rightarrow \mathbb{R}$ is a perfect pairing. Choose $\psi \in U$ such that $\langle\psi, \mathscr{Z}\rangle \subseteq \mathbb{R}_{\geq 0}$ and $P=\mathscr{Z} \cap \psi^{\perp}$, so $J=\left\{j \in I \mid \delta_{j} \in \psi^{\perp}\right\}$. Note that $\left\langle\psi, \Pi_{i}\right\rangle=0$ for $i \in J$ and $\left\langle\psi, \Pi_{i} \backslash \Gamma_{i}\right\rangle \subseteq \mathbb{R}_{>0}$ for $i \in I \backslash J$ since $\delta_{i}$ is a strictly positive linear combination of all the elements of $\Pi_{i},\left|\Pi_{i} \backslash \Gamma_{i}\right| \leq 1$ and $\left\langle\psi, \Gamma_{i}\right\rangle=0$. For each $i \in I \backslash J$, choose $\gamma_{i} \in \mathbb{R} \Gamma_{i}$ such that $\left\langle\gamma_{i}, \Delta_{i}\right\rangle=0$ and $\left\langle\gamma_{i}, \Gamma_{i} \backslash \Delta_{i}\right\rangle \subseteq \mathbb{R}_{>0}$ (which is possible since $\Gamma_{i}$ is linearly independent and $\langle-,-\rangle$ is positive definite on $\mathbb{R} \Gamma_{i}$ ). Let $\phi:=\psi+\epsilon \sum_{i \in I \backslash J} \gamma_{i}$ where $\epsilon>0$. It is easy to see that for sufficiently small $\epsilon>0,\langle\phi, \Pi\rangle \subseteq \mathbb{R}_{\geq 0}$ and $\Pi \cap \phi^{\perp}=\cup_{i \in I} \Delta_{i}=\Delta$. Hence the set $\Delta$ as in (a) is facial.

8.7. From Proposition 3.2 (a), we have $\left\langle z, z^{\prime}\right\rangle \leq 0$ for all $z, z^{\prime} \in \mathscr{Z}$. Since each element $z^{\prime}$ of $\mathscr{Z}^{\mathrm{c}}$ is $W$-conjugate to an element $k$ of $\mathscr{K}^{\mathrm{c}}$, the result below determines the pairs $z, z^{\prime} \in \mathscr{Z}^{\mathrm{c}}$ with $\left\langle z, z^{\prime}\right\rangle=0$ up to $W$-conjugacy.

Lemma. Let $k \in \mathscr{K}^{\mathrm{c}}$ and $z \in \mathscr{Z}^{\mathrm{c}}$ both be non-zero. Let $\Gamma, \Delta$ be connected supports of $k$ and $z$ respectively, chosen so $\Delta$ contains some connected support of the unique element of $W z \cap \mathscr{K}$ (which is possible by Lemma 8.4(i)). Then $\langle z, k\rangle=0$ if and only if one or both of the following two conditions holds:

(i) $\Delta$ and $\Gamma$ are separated.

(ii) $\Gamma$ is of affine type and $z, k$ both span the isotropic ray $\mathbb{R}_{\geq 0} \delta$ of $\Gamma$.

Proof. The "if" direction is trivial. For the converse, suppose $\langle z, k\rangle=0$. Note that neither $\Gamma$ nor $\Delta$ is of finite type, by 8.5(b). Write $z=\sum_{\alpha \in \Delta} c_{\alpha} \alpha$ where all $c_{\alpha}>0$. We have

$$
0=\langle k, z\rangle=\sum_{\alpha \in \Delta} c_{\alpha}\langle k, \alpha\rangle .
$$

Since all $c_{\alpha}>0$ and $\langle k, \alpha\rangle \leq 0$, it follows that $\langle k, \alpha\rangle=0$ for all $\alpha \in \Delta$. This readily implies that either $\Delta$ is separated from $\Gamma$ or $\Delta$ is contained in $\Gamma$. Suppose 
that (i) doesn't hold, so $\Delta \subseteq \Gamma$. If $\Gamma$ is of indefinite type, 8.4(d) implies that every connected component of $\{\beta \in \Gamma \mid\langle\beta, k\rangle=0\}$ is of finite type. Since $\Delta$ is contained in one of these components, we would have $\Delta$ of finite type contrary to above. Therefore $\Gamma$ must be of affine type. Since $\Delta$ is not of finite type and $\Delta \subseteq \Gamma$, we have $\Gamma=\Delta$ of affine type. But $\langle-,-\rangle$ restricted to $\mathbb{R} \Delta$ is positive semi-definite with radical $\mathbb{R} \delta$. Since $\langle k, k\rangle=\langle z, z\rangle \leq 0$ and $k, z \in \mathbb{R}_{\geq 0} \Gamma$, we conclude that (ii) holds.

8.8. The following description of isotropic rays in $\mathscr{Z}$ with connected support is an immediate corollary (cf. [34, Prop 5.7]).

Corollary. Any non-zero element $\delta$ of $\mathscr{Z}^{\mathrm{c}}$ is isotropic if and only if it is $W$ conjugate to a representative of the isotropic ray of some irreducible affine standard parabolic subsystem of $\Phi$.

\section{Hyperbolic and Universal CoXeter groups}

In this section, we discuss the imaginary cone and its closure in the case of "hyperbolic" and "universal" root systems. These two classes of root system play an important role in the study of the imaginary cone in general. We also mention some open questions.

9.1. Consider the following conditions on $(V,\langle-,-\rangle)$ and $(\Phi, \Pi)$.

(i) The restriction of $\langle-,-\rangle$ to $\mathbb{R} \Pi$ has signature $(n, 1,0)$ for some $n \in \mathbb{N}_{\geq 1}$ (i.e. $\mathbb{R} \Pi$ is the orthogonal direct sum of a positive definite subspace of dimension $n \geq 1$ and a negative definite subspace of dimension 1 ).

(ii) No non-empty proper facial subset of $\Pi$ has all its irreducible components of indefinite type.

(iii) Every proper facial subset of $\Pi$ has each of its connected components of finite or affine type.

(iv) Every proper facial subset of $\Pi$ has each of its connected components of finite type.

Clearly (iv) implies (iii) which in turn implies (ii). We shall say that the root system $\Phi$ (or Coxeter group $W$ ) is weakly hyperbolic if (i) holds (in case $V=\mathbb{R} \Pi$, this was called hyperbolic in [34, 4.5-4.6] and [31). We say that $(W, S)$ is hyperbolic if (i) and (iii) hold, and that $(W, S)$ is compact hyperbolic if (i) and (iv) hold.

9.2. If $W$ is weakly hyperbolic, let $\Pi_{1}, \ldots, \Pi_{m}$ be the irreducible components of $\Pi$. We have $\mathbb{R} \Pi=\sum_{i} \mathbb{R} \Pi_{i}$ where $\mathbb{R} \Pi_{i} \perp \mathbb{R} \Pi_{j}$ if $i \neq j$. Moreover, $\mathbb{R} \Pi_{i} \cap \sum_{j \neq i} \mathbb{R} \Pi_{j}$ is in the radical $\{0\}$ of $\langle-,-\rangle$ restricted to $\mathbb{R} \Pi$, so in fact $\mathbb{R} \Pi=\oplus_{i} \mathbb{R} \Pi_{i}$, an orthogonal direct sum. By considering signatures of the restrictions of $\langle-,-\rangle$ to $\mathbb{R} \Pi_{i}$, it follows that all but one of the $\Pi_{i}$ are of finite type and one of them, say $\Pi_{1}$, is of indefinite type. By 8.5(c), $\Pi_{1}$ is a facial subset of $\Pi$. If (ii) holds as well, then $\Pi_{1}=\Pi$ i.e. $\Pi$ is irreducible. In particular, if $W$ is hyperbolic, it is irreducible of indefinite type.

Note that if $W$ is weakly hyperbolic, the Witt index (dimension of a maximal totally isotropic subspace) of the restriction of $\langle-,-\rangle$ to $\mathbb{R} \Pi$ is 1 . This implies that any non-zero totally isotropic face (cf. 7.10 of $\bar{Z}$ is one-dimensional (i.e. a ray), which makes the structure of $\overline{\mathscr{Z}}, R_{0}$ etc much simpler than in general.

Remarks. If $V=\mathbb{R} \Pi$, the notions of hyperbolic and compact hyperbolic above essentially coincide with those in [4, 32] and 33] for those (special) root systems 
which are both of the type here and of the type considered in these sources (which all assume in particular that simple roots are linearly independent).

9.3. The following corollary of Proposition 5.8 is required below.

Corollary. Suppose that $(W, S)$ is irreducible and satisfies the condition 9.1(ii) (as well as 4.1(i)-(iii)). Then $\mathscr{K}=-\mathscr{C} \cap \mathbb{R} \Pi$ and $\mathscr{Z}=-\mathscr{X} \cap \mathbb{R} \Pi$.

Proof. Under the assumptions here, $F_{\mathrm{m}}$.ind $=\emptyset$. Proposition 5.8 (a) therefore gives $\mathscr{K}=-\mathscr{C} \cap \mathbb{R} \Pi$. Since $W \mathbb{R} \Pi=\mathbb{R} \Pi$, this implies

$$
\mathscr{Z}=\bigcup_{w \in W} w(-\mathscr{C} \cap \mathbb{R} \Pi)=\mathbb{R} \Pi \cap \bigcup_{w \in W} w(-\mathscr{C})=\mathbb{R} \Pi \cap-\mathscr{X} .
$$

9.4. If $W$ is weakly hyperbolic on $V$ there is a basis $x_{0}, \ldots, x_{n}$ of $V$ such that $\left\langle x_{i}, x_{j}\right\rangle=0$ for $i \neq j,\left\langle x_{i}, x_{i}\right\rangle=1$ for $i=1, \ldots n$ and $\left\langle x_{0}, x_{0}\right\rangle=-1$.

Let $\mathscr{L}=\left\{\sum_{i} \lambda_{i} x_{i} \mid \lambda_{0}>\sqrt{\sum_{i=1}^{n} \lambda_{i}^{2}}\right\}$; this is an open convex cone in $V$ with closure $\overline{\mathscr{L}}=\left\{\sum_{i} \lambda_{i} x_{i} \mid \lambda_{0} \geq \sqrt{\sum_{i=1}^{n} \lambda_{i}^{2}}\right\}$ satisfying $\overline{\mathscr{L}}^{*}=-\overline{\mathscr{L}}$. (These wellknown assertions follow readily from the Cauchy-Schwarz inequality). The cones $\mathscr{L}$ and $-\mathscr{L}$ are the two connected components of $\{v \in V \mid\langle v, v\rangle<0\}$. We have $0 \neq \mathscr{Z} \subseteq \overline{\mathscr{L}} \cup-\overline{\mathscr{L}}$. Replacing $x_{n}$ by $-x_{n}$ if necessary, assume $\mathscr{Z} \cap \overline{\mathscr{L}} \neq\{0\}$.

Proposition. Let $W$ be irreducible and weakly hyperbolic, and let $\mathscr{L}$ be as defined above. Assume $V=\mathbb{R} \Pi$. Then $\mathscr{L}$ is $W$-invariant and

(a) $(37$, 33, (5.10.2)], 31, Proposition 3.7(i)]) $\mathscr{Z} \subseteq \overline{\mathscr{L}}$.

(b) (37, [31, Proposition 3.7(ii)] $-\mathscr{L} \subseteq \mathscr{X}$.

(c) (compare [33, Ex 5.15]) If the condition 9.1(ii) holds (e.g. W is hyperbolic), then $\mathscr{L} \subseteq \mathscr{Z}=-\mathscr{X} \subseteq \overline{\mathscr{L}}$ and $\overline{\mathscr{Z}}=-\overline{\mathscr{X}}=\mathscr{X}^{*}=\overline{\mathscr{L}} \subseteq \mathbb{R}_{>0} \Pi$. Further, $\mathscr{Z} \backslash \mathscr{L}$ is the set of all isotropic elements of $\mathscr{Z}$.

(d) If $W$ is compact hyperbolic, then $\mathscr{Z}=-\mathscr{X}=\mathscr{L} \cup\{0\}$.

Proof. Let $\mathscr{L}^{\prime}:=\overline{\mathscr{L}} \backslash\{0\}$, so the connected components of $\mathscr{L}^{\prime \prime}:=\{v \in V \backslash\{0\} \mid$ $\langle v, v\rangle \leq 0\}$ are $\pm \mathscr{L}^{\prime}$. By Proposition 3.2, $\mathscr{Z} \backslash\{0\}$ is a connected subset of $\mathscr{L}^{\prime \prime}$ which intersects $\mathscr{L}^{\prime}$ nontrivially, so $\mathscr{Z} \backslash\{0\} \subseteq \mathscr{L}^{\prime}$ and (a) is proved. The $W$-action preserves $\mathscr{L}^{\prime \prime}$ but cannot interchange the two components $\pm \mathscr{L}^{\prime}$ since it preserves $\mathscr{Z} \backslash\{0\} \subseteq \mathscr{L}^{\prime}$. Therefore $\mathscr{L}^{\prime}$ (and hence its interior $\mathscr{L}$ ) is $W$-invariant. Taking duals in (a) gives $-\overline{\mathscr{L}} \subseteq \mathscr{Z}^{*}=\overline{\mathscr{X}}$ by Theorem 5.1. Hence the interior $-\mathscr{L}$ of $-\overline{\mathscr{L}}$ is contained in the interior of $\overline{\mathscr{X}}$, which is in turn contained in $\mathscr{X}$ since $\mathscr{X}$ is a cone. This proves (b).

Assume from now that 9.1(ii) holds. The first part of (c) follows from (a)-(b), Lemma 9.3 and Theorem 5.1 (together with the definition of $\mathscr{Y}$ ). The second part of (c) is trivial since $\overline{\mathscr{L}} \backslash \mathscr{L}$ is the set of all isotropic elements of $\overline{\mathscr{L}}$. Now the isotropic elements of $\mathscr{Z}$ are $W$-conjugate to the isotropic elements of $\mathscr{K}$, which are completely described in Lemma 8.4. In particular, there are no non-zero isotropic elements of $\mathscr{Z}$ unless there is a (necessarily proper since $W$ is of indefinite type) facial subset $I$ of $S$ such that $\Pi_{I}$ has only affine components. Hence (d) follows from the definitions and (c).

9.5. The imaginary cone $\mathscr{Z}_{W^{\prime}}$ of a reflection subgroup $W^{\prime}$ is not in general the intersection of $\mathscr{Z}$ with $\mathbb{R}_{W^{\prime}}$, as simple examples show. However, the next Lemma describes some important special situations in which this is true (in addition to those in Corollary 3.5. 
Corollary. Let $W^{\prime}$ be a finitely generated reflection subgroup of $W$ which is either finite, irreducible affine or hyperbolic (e.g. any dihedral reflection subgroup). Then $\mathscr{Z}_{W^{\prime}}=\mathscr{Z} \cap \mathbb{R} \Pi_{W^{\prime}}$ and $\overline{\mathscr{Z}_{W^{\prime}}}=\overline{\mathscr{Z}} \cap \mathbb{R} \Pi_{W^{\prime}}$.

Proof. Let $L_{W^{\prime}}^{\prime}:=\left\{z \in \mathbb{R}_{W^{\prime}} \mid\langle z, z\rangle \leq 0\right\}$ and $L_{W^{\prime}}:=L_{W^{\prime}}^{\prime} \cap \mathbb{R}_{\geq 0} \Pi_{W^{\prime}}$. We first prove that

$$
L_{W^{\prime}}^{\prime}=L_{W^{\prime}} \cup-L_{W^{\prime}}, \quad \overline{\mathscr{Z}_{W^{\prime}}}=L_{W^{\prime}}
$$

by considering cases according to the type of $W^{\prime}$. If $W^{\prime}$ is finite, then $\overline{\mathscr{Z}_{W^{\prime}}}=0$ by 4.5 and $3.2(\mathrm{~d})$, while $L_{W^{\prime}}=L_{W^{\prime}}^{\prime}=0$ by Lemma 2.9. If instead $W^{\prime}$ is affine, then by $4.4, \mathscr{Z}_{W^{\prime}}=\mathscr{Z}_{W^{\prime}}=\mathbb{R}_{\geq 0} \delta$ (where $\delta$ spans the isotropic ray of $W^{\prime}$ ), $L_{W^{\prime}}=\mathbb{R}_{\geq 0} \delta$ and $L_{W^{\prime}}^{\prime}=\mathbb{R} \delta$. Finally, if $W^{\prime}$ is of hyperbolic type, then 9.5.1) follows from Proposition 9.4(c). Next, we claim that

$$
L_{W^{\prime}}^{\prime} \cap \mathbb{R}_{\geq 0} \Pi=L_{W^{\prime}}, \quad \mathscr{K}_{W^{\prime}}=-\mathscr{C}_{W^{\prime}} \cap L_{W^{\prime}} .
$$

The first equation above follows directly from the first equation in 9.5 .1 , and the second follows from the definitions and Proposition 3.2(c).

Now by Lemma 3.2 (c), $\overline{\mathscr{Z}} \cap \mathbb{R} \Pi_{W^{\prime}} \subseteq L_{W^{\prime}}^{\prime}$. We get from Theorem 6.3 that

$$
\overline{\mathscr{Z}_{W^{\prime}}} \subseteq \overline{\mathscr{Z}} \cap \mathbb{R}_{W^{\prime}} \subseteq L_{W^{\prime}}^{\prime} \cap \mathbb{R}_{\geq 0} \Pi \subseteq L_{W^{\prime}}=\overline{\mathscr{Z}_{W^{\prime}}}
$$

by (9.5.1)-9.5.2). Next, note that $\mathscr{Z}_{W^{\prime}} \subseteq \mathscr{Z} \cap \mathbb{R} \Pi_{W^{\prime}}$ by 6.3 again. To prove the reverse inclusion, let $z \in \mathscr{Z} \cap \mathbb{R}_{W^{\prime}}$. Choose $w \in W$ with $w z \in \mathscr{K}$, and let $W^{\prime \prime}:=w W^{\prime} w^{-1}$ which is a reflection subgroup of $W$ of the same type (finite, irreducible affine or hyperbolic) as $W^{\prime}$. Using Proposition 3.2(c) for $W$, and 9.5 .2 . for $W^{\prime \prime}$, we have

$$
w z \in \mathscr{K} \cap \mathbb{R}_{W^{\prime \prime}}=-\mathscr{C} \cap \mathbb{R}_{\geq 0} \Pi \cap L_{W^{\prime \prime}}^{\prime} \subseteq-\mathscr{C}_{W^{\prime \prime}} \cap L_{W^{\prime \prime}}=\mathscr{K}_{W^{\prime \prime}} \subseteq \mathscr{Z}_{W^{\prime \prime}}
$$

and so $z \in w^{-1} \mathscr{Z}_{W^{\prime \prime}}=\mathscr{Z}_{W^{\prime}}$ as required.

9.6. Some questions. We next collect some (mostly open) questions which have arisen in the course of this work or are suggested by study of examples. One preliminary remark is that some properties of the closed imaginary cone which hold for irreducible root systems fail trivially for reducible ones. Correspondingly, even for irreducible $W$, points of this cones may fail to have some property of interest if the point is conjugate to a point in the cone attached to a proper facial parabolic subgroup (since the latter may be reducible). Therefore define the set of generic points of $\overline{\mathscr{Z}}$ to be

$$
\overline{\mathscr{Z}}_{\text {gen }}:=\left\{\alpha \in \overline{\mathscr{Z}} \mid w \alpha \notin \overline{\mathscr{Z}}_{I} \text { for all } w \in W \text { and facial } I \subsetneq S\right\} .
$$

(We don't make a corresponding definition for $\mathscr{Z}$ since it just yields the relative interior of $\mathscr{Z}$, as follows from results in $\$ 10$ )

The following definitions are more naturally formulated in terms of a compact convex base of a closed salient cone as in Lemma 7.10, but for uniformity, we continue in terms of cones. Let $C$ be any closed salient cone in $V$. For $p \in V$, set $C(p):=\operatorname{cone}(C-p)$. Fix non-zero $p \in \operatorname{rb}(C)$. Call $p$ a smooth boundary point if there is a unique absolutely supporting linear hyperplane for $C$ in $\mathbb{R} C$ which contains $p$. Say that $p$ is a round boundary point if there is some (necessarily unique) linear hyperplane $H$ in $\mathbb{R} C$ containing the line $\mathbb{R} p$ such that $C(p)$ is the union of $\mathbb{R} p$ with one of the two open halfspaces in $\mathbb{R}(C-p)$ determined by $H$. Finally, say that $p$ is a flat boundary point if it is in the relative interior of an 
exposed face of $C$ of codimension 1. Note that round or flat boundary points are smooth, but not conversely.

Question. Let $(W, S)$ be an irreducible finite rank Coxeter system of indefinite type.

(a) Is $\overline{\mathscr{Z}}_{\text {gen }} \subseteq \mathscr{Z} \cup \mathscr{Q}$ ? More strongly, is every proper face $F$ of $\overline{\mathscr{Z}}$ such that $\operatorname{ri}(F) \cap \overline{\mathscr{Z}}_{\text {gen }} \neq \emptyset$ totally isotropic?

(b) (See 7.9 Is $R_{0}=\bar{Z} \cap Q$ (raised by Hohlweg and Ripoll)? If not, is it at least true that $\overline{\mathscr{Z}}_{\text {gen }} \cap \mathscr{Q} \subseteq \cup R_{0}$ ?

(c) Is $\overline{\mathscr{Z}}$ equal to the topological closure of the convex hull of the union of the set of its round boundary points and $\{0\}$ ?

(d) Is every extreme ray of $\overline{\mathscr{Z}}$ exposed (cf. Lemma A.11(b)). More generally, is every face of $\overline{\mathscr{Z}}$ exposed? Is it even true that $(\mathscr{Z}, \overline{\mathscr{X}})$ is a dual pair of stable cones (cf. Theorem 10.3)?

(e) Do any two distinct, maximal, totally isotropic faces of $\overline{\mathscr{Z}}$, such that each of them contains some point of $\overline{\mathscr{Z}}_{\text {gen }}$, intersect in $\{0\}$ ?

Remarks. (1) Results in 21] are relevant to the study of several of these questions. It should not be difficult to settle some of them for hyperbolic groups (for example, for hyperbolic $\Phi$, one has $\overline{\mathscr{Z}} \subseteq \mathscr{Z} \cup \mathscr{Q}$, more strongly than (a), by Proposition 9.4. See 9.18 for an example showing the necessity of restrictions in (a), (b), (e) for non-hyperbolic $\Phi$.

(2) There is considerable diversity even in the imaginary cones of rank three universal Coxeter groups. Depending on the root system, $\operatorname{rb}(\mathscr{Z}) \backslash\{0\}$ may either consist entirely of round boundary points and be a differentiable submanifold of $V$ or contain no round boundary point which has a neighborhood in $\operatorname{rb}(\mathscr{Z}) \backslash\{0\}$ which is a differentiable submanifold of $V$. Similarly in this case, $R_{0}$ may be topologically a circle or a Cantor set.

9.7. It would be natural to study the dynamics of the action of special elements of $W$ and subgroups of $W$ on the closed imaginary cone, the disposition of eigenvectors and eigenspaces in relation to the faces etc. The following question in this vein is suggested by Lemma 6.1(f).

Question. If $\Phi$ is irreducible of indefinite type and $\alpha \in \overline{\mathscr{Z}}_{\text {gen }} \cap \mathscr{Q}$, is $0 \in \overline{W \alpha}$.

The condition that $\alpha$ be generic is included to exclude possibilities like $\alpha=$ $\alpha^{\prime}+\alpha^{\prime \prime}$ where $\alpha^{\prime} \in \mathscr{Z}$ and $\alpha^{\prime \prime} \in \overline{\mathscr{Z}} \cap \mathscr{Q}$ have separated facial supports and $\alpha^{\prime}$ spans the isotropic ray of an affine standard parabolic subsystem; in such a case, $0 \in \overline{W \alpha}$ is not possible.

9.8. Other natural questions involve ubiquity of reflection subgroups which are universal Coxeter groups, and their properties. Note that the based root system $(\Phi, \Pi)$ is the root system of a universal Coxeter group if and only if $\langle\alpha, \beta\rangle \leq-1$ for all distinct $\alpha, \beta \in \Pi$. In this case, we also say that $(\Phi, \Pi)$ is universal. Using [18. (see [13, Remark 3.12]), it is equivalent to require $|\langle\alpha, \beta\rangle| \geq 1$ for all $\alpha, \beta$ in $\Phi$. We shall say that $(\Phi, \Pi)$ is a generic universal based root system if $\langle\alpha, \beta\rangle<-1$ for all distinct $\alpha, \beta \in \Pi$. (Using Corollary 8.8 this is easily seen to be equivalent to $|\langle\alpha, \beta\rangle|>1$ for all $\alpha, \beta \in \Phi$ with $\beta \neq \pm \alpha$, but we shall not need this fact).

Assume that $(W, S)$ is an irreducible Coxeter system of indefinite type and of finite rank at least two. We define a metric on the set of non-zero closed pointed 
cones which are contained in $\mathbb{R}_{\geq 0} \Pi$ by using a Hausdorff metric (see e.g. [51]) on their intersections with $\left\{v \in \mathbb{R}_{\geq 0} \Pi \mid\langle v, \rho\rangle=1\right\}$ (which is a compact convex base of $\left.\mathbb{R}_{\geq 0} \Pi\right)$.

Question. Does there exist a finite rank reflection subgroup $W^{\prime}$ of $W$ such that $\left(\Phi_{W^{\prime}}, \Pi_{W^{\prime}}\right)$ is a generic universal based root system and $\mathbb{R} \Pi_{W^{\prime}}=\mathbb{R} \Pi$. Is there a sequence of such reflection subgroups $W_{n}^{\prime}, n \in \mathbb{N}$, such that both sequences $\mathbb{R}_{\geq 0} \Pi_{W_{n}^{\prime}}$ and $\overline{\mathscr{Z}_{W_{n}^{\prime}}}$, for $n \in \mathbb{N}$, converge to $\overline{\mathscr{Z}}$ in the above metric?

This was established for hyperbolic groups in 22]. It will be shown in [21] that the question has an affirmative answer; in fact, we will prove more general results about approximation of non-zero faces of $\overline{\mathscr{Z}}$.

9.9. Generic universal root systems. Because of the general importance of generic universal root systems in view of the affirmative answer in [21] to the previous question, we discuss some of their properties in the remainder of this section.

Assumption. In 9.10 9.17, it is assumed unless otherwise stated (in addition to the standing assumptions $4.1(\mathrm{i})$-(iii)) that $(\Phi, \Pi)$ is generic universal (i.e. $\langle\alpha, \beta\rangle<-1$ for all $a \neq \beta \in \Pi)$ and, to avoid trivialities, that $|\Pi| \geq 2$.

Give the finite set $\Pi$ the discrete topology and $\prod_{n \in \mathbb{N}} \Pi$ the product topology. Let $C$ the the closed (topological) subspace of $\prod_{n \in \mathbb{N}} \Pi$ consisting of all sequences $\beta=\left(\beta_{n}\right)_{n \in \mathbb{N}}$ in $\Pi$ with $\beta_{i} \neq \beta_{i+1}$ for all $i$. In general, $C$ is a compact, totally disconnected, metrizable space. If $|\Pi|>2$, then $C$ is perfect (i.e. has no isolated points) and it is well known that these conditions then imply that $C$ is a Cantor space i.e. it is homeomorphic to the (standard, ternary) Cantor set. If $|\Pi|=2$, then $C$ is a discrete two-point space. For any $\beta=\left(\beta_{n}\right)_{n \in \mathbb{N}} \in C$, set

$$
F_{\beta}:=\left\{z \in \overline{\mathscr{Z}} \mid \lim _{n \rightarrow \infty} s_{\beta_{n-1}} \cdots s_{\beta_{0}} z=0\right\} .
$$

The theorem below is our main result on generic universal root systems. It shows in particular that $\overline{\mathscr{Z}} \backslash \mathscr{Z} \subseteq \mathscr{Q}$ and that the space of connected components of $\overline{\mathscr{Z}} \backslash \mathscr{Z}$ (its quotient space identifying the components to points) is homeomorphic to $C$.

Theorem. $\quad$ (a) $\mathscr{Z} \cap \mathscr{Q}=\{0\}$ and $\overline{\mathscr{Z}} \backslash \mathscr{Z} \subseteq \mathscr{Q}$.

(b) For $\beta=\left(\beta_{n}\right)_{n \in \mathbb{N}} \in C, \overline{\mathscr{Z}} \backslash F_{\beta}=\left\{z \in \overline{\mathscr{Z}} \mid \lim _{n \rightarrow \infty}\left\langle\rho, s_{\beta_{n-1}} \cdots s_{\beta_{0}} z\right\rangle=\right.$ $+\infty\}$.

(c) There is a well-defined function $b: \overline{\mathscr{Z}} \backslash \mathscr{Z} \rightarrow C$ defined by setting $b(z):=\beta$ if $\beta \in C$ and $z \in F_{\beta} \backslash\{0\}$.

(d) The fibers of $b$, namely the sets $b^{-1}(\beta)=F_{\beta} \backslash\{0\}$, for $\beta \in C$, are the connected components of $\overline{\mathscr{Z}} \backslash \mathscr{Z}$.

(e) $\left\{F_{\beta} \mid \beta \in C\right\}$ is the set of all faces of $\overline{\mathscr{Z}}$ which are maximal in the set of faces $F$ of $\overline{\mathscr{Z}}$ satisfying $F \cap \mathscr{Z}=\{0\}$. It is also the set of all maximal totally isotropic faces of $\overline{\mathscr{Z}}$.

(f) The map $b$ is a quotient map. It factors as a composite $b=q p$ where $p: \overline{\mathscr{Z}} \backslash \mathscr{Z} \rightarrow \operatorname{ray}(\mathscr{Q} \cap \overline{\mathscr{Z}})$ is an open quotient map $\alpha \mapsto \mathbb{R}_{>0} \alpha$ and where $q: \operatorname{ray}(\mathscr{Q} \cap \overline{\mathscr{Z}}) \rightarrow C$ is a closed quotient map given by $\mathbb{R}_{\geq 0} \alpha \mapsto b(\alpha)$.

Subsections 9.10 9.17 give the proof of the theorem, and related facts. 
9.10. Some of the following results, especially the early ones, can be generalized, with more technical statements, to hold for all universal $(\Phi, \Pi)$. We shall find it convenient to use again the notation $H_{\phi}^{\prec}$ introduced in 2.3 . Also, for any non-zero $v \in \mathbb{R}_{\geq 0} \Pi$, write $\widehat{v}:=\frac{1}{\langle\rho, v\rangle} v$ where we have fixed $\rho \in V$ with $\langle\rho, \Pi\rangle \subseteq \mathbb{R}_{\geq 0}$. Set $\epsilon_{\rho}:=\min (\{\langle\rho, \beta\rangle \mid \beta \in \Pi\})>0$. Throughout the proof, whenever multiple root systems are under consideration and it is necessary to indicate notationally the dependence of some object we have defined on the root system $(\Phi, \Pi)$, we shall do so by attaching a subscript $\Pi$ (or $W$ ).

For any distinct $\alpha$ and $\beta$ in $\Pi$, write $\langle\alpha, \beta\rangle=-\cosh \lambda$ where $\lambda=\lambda_{\alpha, \beta}>0$. Let $u_{\Pi}(\alpha, \beta)=u(\alpha, \beta):=e^{\lambda} \alpha+\beta \in \mathscr{Q}$ and $u_{\Pi}^{\prime}(\alpha, \beta)=u^{\prime}(\alpha, \beta):=(\cosh \lambda) \alpha+\beta \in \alpha^{\perp}$. It is easily verified from our general results (or by direct computation using for instance the discussion of dihedral root systems in [30]), that the imaginary cone of $W^{\prime}=W_{\alpha, \beta}=\left\langle s_{\alpha}, s_{\beta}\right\rangle$ is $\mathscr{Z}_{W^{\prime}}=\mathbb{R}_{>0}\{u(\alpha, \beta), u(\beta, \alpha)\} \cup\{0\}$, with closure $\overline{\mathscr{Z}}_{W^{\prime}}=\mathbb{R}_{\geq 0}\{u(\alpha, \beta), u(\beta, \alpha)\}$ and the fundamental domain for $W^{\prime}$ on $\mathscr{Z}_{W^{\prime}}$ is $\mathscr{K}_{W^{\prime}}=\mathbb{R}_{\geq 0}\left\{u^{\prime}(\alpha, \beta), u^{\prime}(\beta, \alpha)\right\}$. Also define

$$
\mathscr{K}^{+}:=\mathbb{R}_{\geq 0}\{u(\alpha, \beta) \mid \alpha, \beta \in \Pi, \alpha \neq \beta\}=\mathbb{R}_{\geq 0}\left(\bigcup_{\alpha \neq \beta \in \Pi} \overline{\mathscr{Z}}_{W_{\{\alpha, \beta\}}}\right) \subseteq \overline{\mathscr{Z}} .
$$

It is important for our purposes to understand the relative position of the rays through the points

$$
\begin{array}{ll}
x_{1}:=\alpha & x_{2}:=u(\alpha, \beta)=e^{\lambda} \alpha+\beta \\
x_{3}:=u^{\prime}(\alpha, \beta)=(\cosh \lambda) \alpha+\beta & x_{4}:=u^{\prime}(\beta, \alpha)=\alpha+(\cosh \lambda) \beta \\
x_{5}:=u(\beta, \alpha)=\alpha+e^{\lambda} \beta & x_{6}:=\beta
\end{array}
$$

where $\alpha \neq \beta \in \Pi$. Define the slope of $u:=a \alpha+b \beta$, where $a, b \in \mathbb{R}_{\geq 0}$ are not both zero, as $m(u)=\frac{b}{a} \in \mathbb{R}_{\geq 0} \cup\{\infty\}$. Then

$$
\begin{aligned}
m\left(x_{1}\right)=0<m\left(x_{2}\right)=e^{-\lambda}< & m\left(x_{3}\right)=\frac{1}{\cosh \lambda} \\
<m\left(x_{4}\right) & =\cosh (\lambda)<m\left(x_{5}\right)=e^{\lambda}<m\left(x_{6}\right)=\infty .
\end{aligned}
$$

This implies that

$$
\mathbb{R}_{\geq 0} x_{j} \subseteq \mathbb{R}_{\geq 0} x_{i}+\mathbb{R}_{\geq 0} x_{k} \Longleftrightarrow i \leq j \leq k, \quad \text { if } 1 \leq i \leq k \leq 6,1 \leq j \leq 6 .
$$

Similar results are formulated in an affine setting in 30; the condition in (9.10.1) is equivalent to $\widehat{x}_{j} \in \operatorname{conv}\left(\left\{\widehat{x}_{i}, \widehat{x}_{j}\right\}\right)$ and the result is represented by the diagram

$$
-\widehat{x}_{1}-\widehat{x}_{2}-\widehat{x}_{3}-\widehat{x}_{4}-\widehat{x}_{5}-\widehat{x}_{6}-
$$

of points on the affine line spanned by the $\widehat{x}_{i}$.

9.11. The lemma below describes basic relations between $\mathscr{K}, \mathscr{K}^{+}, \overline{\mathscr{Z}}$ and $\mathscr{Q}$.

Lemma. (a) $\mathscr{K}=\mathbb{R}_{\geq 0}\left\{u^{\prime}(\alpha, \beta) \mid \alpha \neq \beta \in \Pi\right\}=\mathbb{R}_{\geq 0}\left(\bigcup_{\alpha \neq \beta \in \Pi} \mathscr{K}_{W_{\{\alpha, \beta\}}}\right)$.

(b) $\mathbb{R}_{\geq 0} \Pi=\mathscr{K} \dot{\cup}\left(\dot{U}_{\alpha \in \Pi}\left(\mathbb{R}_{\geq 0} \Pi \cap H_{\alpha}^{>}\right)\right)$.

(c) $\mathscr{Z} \cap \mathscr{Q}=\{0\}$.

(d) $\mathscr{K} \subseteq \mathscr{K}^{+} \subseteq \overline{\mathscr{Z}}$.

(e) $\overline{\mathscr{Z}}=\overline{\cup_{w \in W} w \mathscr{K}^{+}}$. 
Proof. We first prove (a)-(b) assuming that $\Pi$ is linearly independent. By definition, $\mathscr{K}=\mathbb{R}_{\geq 0} \Pi \cap \bigcap_{\alpha \in \Pi} H_{\alpha}^{\leq}$. One easily checks

$$
\mathbb{R}_{\geq 0} \Pi \cap H_{\alpha}^{=}=\mathbb{R}_{\geq 0}\left\{u_{\alpha, \beta}^{\prime} \mid \beta \in \Pi \backslash\{\alpha\}\right\} .
$$

Also, for any $\alpha \in \Pi, H_{\alpha}^{<}$does not contain $\alpha$, and it contains $u^{\prime}(\beta, \gamma)$, where $\beta \neq \gamma$ in $\Pi$, if and only if $\beta \neq \alpha$. It follows that $\left\{u^{\prime}(\alpha, \beta) \mid \alpha, \beta \in \Pi, \alpha \neq \beta\right\}$ is a set of representatives for the extreme rays of the polyhedral cone $\mathscr{K}$, proving the first equality in (a). The second equality in (a) is clear from the formulae in 9.10. Recall that the facets of $\mathscr{K}$ are its codimension one faces. These are the cones

$$
\mathscr{K} \cap \mathbb{R}_{\geq 0}(\Pi \backslash\{\alpha\})=\mathbb{R}_{\geq 0}\left\{u_{\beta, \gamma}^{\prime} \mid \beta, \gamma \in \Pi \backslash\{\alpha\}, \beta \neq \gamma\right\}
$$

for $\alpha \in \Pi$ (provided $|\Pi| \geq 3$ ) and $\mathscr{K} \cap \alpha^{\perp}=\mathbb{R}_{\geq 0} \Pi \cap H_{\alpha}^{=}$for $\alpha \in \Pi$. Moreover,

$$
\mathbb{R}_{\geq 0} \Pi \cap H_{\alpha}^{\geq 0}=\mathbb{R}_{\geq 0}\left(\{\alpha\} \cup\left\{u_{\alpha, \beta}^{\prime} \mid \beta \in \Pi \backslash\{\alpha\}\right\}\right)
$$

for $\alpha \in \Pi$. It is easy to see from these equations that (b) also holds. Next, to prove (a)-(b) in general (if $\Pi$ is is possibly linearly dependent) choose a canonical lift $\left(\Phi^{\prime}, \Pi^{\prime}\right)$ in $\left(V^{\prime},\langle-,-\rangle^{\prime}\right)$ of $(\Phi, \Pi)$ with canonical map $L: V^{\prime} \rightarrow V$ as in 1.4. For any distinct $\alpha^{\prime}, \beta^{\prime} \in \Pi^{\prime}$, one has $L\left(u_{\Pi^{\prime}}^{\prime}\left(\alpha^{\prime}, \beta^{\prime}\right)\right)=u_{\Pi}^{\prime}\left(L\left(\alpha^{\prime}\right), L\left(\beta^{\prime}\right)\right)$ since $\left\langle L\left(\alpha^{\prime}\right), L\left(\beta^{\prime}\right)\right\rangle=\left\langle\alpha^{\prime}, \beta^{\prime}\right\rangle^{\prime}$. Part (a) follows directly by applying $L$ to the formula (a) for $\left(\Phi^{\prime}, \Pi^{\prime}\right)$ (known since $\Pi^{\prime}$ is linearly independent) using Proposition 3.6. Part (b) follows similarly using $\mathbb{R}_{\geq 0} \Pi^{\prime} \cap L^{-1}\left(\mathbb{R}_{\geq 0} \Pi \cap H_{L\left(\alpha^{\prime}\right)}^{>}\right)=\mathbb{R}_{\geq 0} \Pi^{\prime} \cap H_{\alpha^{\prime}}^{>}$in $V^{\prime}$ and $L\left(\mathbb{R}_{\geq 0} \Pi^{\prime} \cap H_{\alpha^{\prime}}^{>}\right)=\mathbb{R}_{\geq 0} \Pi \cap H_{L\left(\alpha^{\prime}\right)}^{>}$in $V$, for $\alpha^{\prime} \in \Pi^{\prime}$.

For (c), note $\mathscr{K}^{\mathrm{c}}=\mathscr{K} \backslash\{0\}$ and $\mathscr{Z}^{\mathrm{c}}=\mathscr{Z} \backslash\{0\}$ since any non-zero element of $\mathbb{R}_{\geq 0} \Pi$ has a connected support (in fact, any non-empty subset of $\Pi$ is connected). Since there are no irreducible affine standard parabolic subsystems of $\Phi$, part (c) holds by Corollary 8.8. In (d) the first (resp., second) inclusion holds since for all $\alpha \neq \beta$ in $\Pi, \mathscr{K}_{W_{\alpha, \beta}} \subset \overline{\mathscr{Z}}_{W_{\alpha, \beta}}$ (resp., $\overline{\mathscr{Z}}_{W_{\alpha, \beta}} \subseteq \overline{\mathscr{Z}}$ ). From (d) and the definition $\mathscr{Z}=\cup_{w \in W} w(\mathscr{K})$, we have

$$
\overline{\mathscr{Z}}=\overline{\cup_{w \in W} w \overline{\mathscr{Z}}} \supseteq \overline{\cup_{w \in W} w \mathscr{K}^{+}} \supseteq \overline{\cup_{w \in W} w \mathscr{K}}=\overline{\mathscr{Z}}
$$

which proves (e).

9.12. The next lemma establishes a crucial special fact about generic universal root systems: the positive root cone is the union of the imaginary cone and certain cones $\mathscr{D}_{\alpha} \backslash\{0\}$, indexed by simple roots $\alpha$, the union of which contains all positive roots and also the intersection of the positive root cone and isotropic cone. This fact plays a significant role in the study of root systems of general Coxeter groups in [21]. For any $\alpha \in \Pi$, define

$$
\mathscr{D}_{\alpha}=\mathscr{D}_{\Pi, \alpha}:=\mathbb{R}_{\geq 0}(\{\alpha\} \cup\{u(\alpha, \beta) \mid \beta \in \Pi \backslash\{\alpha\}\}) \subseteq \mathbb{R}_{\geq 0} \Pi
$$

Lemma. (a) For $\alpha \in \Pi, \mathscr{D}_{\alpha} \backslash\{0\} \subseteq H_{\alpha}^{>} \cap \bigcap_{\beta \in \Pi \backslash\{\alpha\}} H_{\beta}^{<}$. In particular, $\mathscr{D}_{\alpha} \cap \mathscr{D}_{\beta}=\{0\}$ if $\alpha \neq \beta$ in $\Pi$.

(b) There is a constant $k_{\rho}>0$ (depending on $\rho$ ) such that if $\gamma \in \bigcup_{\alpha \in \Pi} \mathscr{D}_{\alpha}$ and $\beta \in \Pi$ then $\left|\left\langle\gamma, \beta^{\vee}\right\rangle\right| \geq k_{\rho}|\langle\rho, \gamma\rangle|$.

(c) There is a constant $k_{\rho}^{\prime}>0$ such that if $\gamma \in \mathscr{D}_{\alpha}$ and $\gamma^{\prime} \in \mathscr{D}_{\beta}$ where $\alpha, \beta \in \Pi$ are distinct, then $\left\langle\gamma, \gamma^{\prime}\right\rangle \leq-k_{\rho}^{\prime}\langle\gamma, \rho\rangle\left\langle\gamma^{\prime}, \rho\right\rangle$.

(d) $\mathbb{R}_{\geq 0} \Pi=\mathscr{Z} \cup\left(\bigcup_{\alpha \in \Pi} \mathscr{D}_{\alpha}\right)$.

(e) $\Phi_{+} \cup\left(\left(\mathscr{Q} \cap \mathbb{R}_{\geq 0} \Pi\right) \backslash\{0\}\right) \subseteq \mathbb{R}_{\geq 0} \Pi \backslash \mathscr{Z} \subseteq \bigcup_{\alpha \in \Pi} \mathscr{D}_{\alpha}$. 
(f) Let $\gamma \in \mathbb{R}_{\geq 0} \Pi \backslash \mathscr{Z}$. Then for $\alpha \in \Pi,\langle\gamma, \alpha\rangle \geq 0$ if and only if $\gamma \in \mathscr{D}_{\alpha}$ if and only if $\langle\gamma, \alpha\rangle>0$. These conditions are satisfied by a unique $\alpha \in \Pi$.

Proof. Part (a) holds since for $\alpha \neq \beta \in \Pi$, one has $\langle\alpha, \alpha\rangle>0$ and $\langle\alpha, u(\alpha, \beta)\rangle>0$ while for $\gamma \in \Pi \backslash\{\alpha\}$, one has $\langle\gamma, \alpha\rangle<0$ and $\langle\gamma, u(\alpha, \beta)\rangle<0$. For (b), note that for any $\alpha, \beta \in \Pi$ and $\gamma \in \mathscr{D}_{\alpha} \backslash\{0\}$, one has $\left|\left\langle\gamma, \beta^{\vee}\right\rangle\right| \geq k_{\alpha, \beta}\langle\gamma, \rho\rangle$ by (a), where

$$
k_{\alpha, \beta}:=\min \left(\left\{\frac{\left|\left\langle\beta^{\vee}, u\right\rangle\right|}{|\langle\rho, u\rangle|} \mid u \in\{\alpha\} \cup\{u(\alpha, \gamma) \mid \gamma \in \Pi \backslash\{\alpha\}\}\right\}\right) .
$$

Part (b) holds setting $k_{\rho}:=\min \left(\left\{k_{\alpha, \beta} \mid \alpha, \beta \in \Pi\right\}\right)$. The proof of (c) is similar to that of (b) and we omit it.

In the proof of (d), assume first that $\Pi$ is linearly independent. Using this assumption, choose for each $\alpha \in \Pi$ an element $\phi_{\alpha} \in V$ such that $\left\langle\phi_{\alpha}, u(\alpha, \beta)\right\rangle=0$ for all $\beta \in \Pi \backslash\{\alpha\}$ and $\left\langle\phi_{\alpha}, \alpha\right\rangle>0$. Let $\alpha \neq \beta$ in $\Pi$. It follows from (9.10.1p that $u(\alpha, \beta) \in \mathbb{R}_{>0}\{\alpha, \gamma\}$ for each $\gamma \in\left\{u^{\prime}(\alpha, \beta), u^{\prime}(\beta, \alpha), u(\beta, \alpha), \beta\right\}$. It follows that $\left\langle\phi_{\alpha}, \gamma\right\rangle<0$ for such $\gamma$. That is, the hyperplane $H_{\phi_{a}}^{=} \cap \mathbb{R} \Pi$ in $\mathbb{R} \Pi$ strictly separates $\alpha$ and $\gamma$. It follows that it also strictly separates $\alpha$ from $\Pi \backslash\{\alpha\}$ and $\alpha$ from $u_{\gamma, \delta}$ for any $\gamma \neq \delta$ in $\Pi \backslash\{\alpha\}$. One easily checks that for any $\alpha \in \Pi$, $\mathbb{R}_{\geq 0} \Pi \cap H_{\phi_{\alpha}}^{=}=\mathbb{R}_{\geq 0}\{u(\alpha, \beta) \mid \beta \in \Pi \backslash\{\alpha\}\}$. An argument very similar to that in the proof of Lemma 9.11.(a) shows that $\mathscr{K}^{+}=\mathbb{R}_{\geq 0} \Pi \cap \bigcap_{\alpha \in \Pi} H_{\phi_{\alpha}}^{\leq}$. The facets of $\mathscr{K}^{+}$are the cones $\mathscr{K}^{+} \cap \mathbb{R}_{\geq 0}(\Pi \backslash\{\alpha\})=\mathbb{R}_{\geq 0}\left\{u_{\beta, \gamma} \mid \beta \neq \gamma \in \Pi \backslash\{\alpha\}\right\}=\mathscr{K}_{\Pi \backslash\{\alpha\}}^{+}$ for $\alpha \in \Pi$ (provided $|\Pi| \geq 3$ ) and $\mathscr{K}^{+} \cap H_{\bar{\phi}_{\alpha}}^{=}=\mathbb{R}_{\geq 0}\left\{u_{\alpha, \beta} \mid \beta \in \Pi \backslash\{\alpha\}\right\}$ for $\alpha \in \Pi$. Also, $\mathscr{D}_{\alpha}=\mathbb{R}_{\geq 0} \Pi \cap H_{\bar{\phi}_{\alpha}}^{>}$. This makes it clear that $\mathbb{R}_{\geq 0} \Pi=\mathscr{K}^{+} \cup\left(\bigcup_{\alpha \in \Pi} \mathscr{D}_{\alpha}\right)$.

Now clearly $\mathbb{R}_{\geq 0} \Pi \supseteq \mathscr{Z} \cup\left(\bigcup_{\alpha \in \Pi} \mathscr{D}_{\alpha}\right)$. We prove the reverse inclusion by induction on $n:=|\Pi|$. If $n=2$, say $\Pi=\{\alpha, \beta\}$, this follows easily from 9.10.1) (in fact, the right hand side is a partition of the left hand side in this case). Now assume $n \geq 3$. From above, it suffices to show $\mathscr{K}^{+} \subseteq \mathscr{Z} \cup\left(\bigcup_{\alpha \in \Pi} \mathscr{D}_{\alpha}\right)$. Let $z \in \mathscr{K}^{+}$. Now clearly $\mathbb{R} \mathscr{K}^{+}=\mathbb{R} \Pi=\mathbb{R} \mathscr{Z}$. Hence $\operatorname{ri}\left(\mathscr{K}^{+}\right) \subseteq \operatorname{ri}(\overline{\mathscr{Z}})=\operatorname{ri}(\mathscr{Z}) \subseteq \mathscr{Z}$. Therefore if $z \in \operatorname{ri}\left(\mathscr{K}^{+}\right)$, then $z \in \mathscr{Z}$ as required. In the contrary case, $z$ is in some facet of $\mathscr{K}^{+}$. Now for $\alpha \in \Phi$, the facet $\mathscr{K}^{+} \cap H_{\bar{\phi}_{\alpha}}=\mathbb{R}_{\geq 0} \cap H_{\phi_{\alpha}}$ is also a facet of $\mathscr{D}_{\alpha}$. Hence we may assume that $z$ is in a facet $\mathscr{K}_{\Delta}^{+}$of $\mathscr{K}^{+}$where $\Delta:=\Pi \backslash\{\alpha\}$ for some $\alpha \in \Pi$. By induction, $z \in \mathscr{Z}_{\Delta} \cup\left(\bigcup_{\beta \in \Delta} D_{\Delta, \beta}\right)$. By Remark 3.5 (2), $\mathscr{Z}_{\Delta}=\mathscr{Z} \cap \mathbb{R}_{\geq 0} \Delta$. Also, $\mathscr{D}_{\Delta, \beta}=\mathbb{R}_{\geq 0}\left(\{\beta\} \cup\left\{u_{\beta, \gamma} \mid \gamma \in \Delta \backslash\{\beta\}\right\}\right)=\mathscr{D}_{\beta} \cap \mathbb{R}_{\geq 0} \Delta$. Therefore $z \in \mathscr{Z} \cup\left(\bigcup_{\beta \in \Pi} \mathscr{D}_{\beta}\right)$ as required to complete the proof of (d) in the case of linearly independent $\Pi$.

If $\Pi$ is linearly dependent, choose a canonical lift $\left(\Phi^{\prime}, \Pi^{\prime}\right)$ in $\left(V^{\prime},\langle-,-\rangle^{\prime}\right)$ of $(\Phi, \Pi)$ with canonical map $L: V^{\prime} \rightarrow V$ as in 1.4 For any distinct $\alpha^{\prime}, \beta^{\prime} \in \Pi^{\prime}$, one has $L\left(u_{\Pi^{\prime}}\left(\alpha^{\prime}, \beta^{\prime}\right)\right)=u_{\Pi}\left(L\left(\alpha^{\prime}\right), L\left(\beta^{\prime}\right)\right)$ since $\left\langle L\left(\alpha^{\prime}\right), L\left(\beta^{\prime}\right)\right\rangle=\left\langle\alpha^{\prime}, \beta^{\prime}\right\rangle^{\prime}$. The definitions therefore give $L\left(\mathscr{D}_{\Pi^{\prime}, \alpha^{\prime}}\right)=\mathscr{D}_{\Pi, L\left(\alpha^{\prime}\right)}$ for $\alpha \in \Pi^{\prime}$. By the linear independence of $\Pi^{\prime}$ and the case previously treated, we therefore get

$$
\begin{aligned}
\mathbb{R}_{\geq 0} \Pi & =L\left(\mathbb{R}_{\geq 0} \Pi^{\prime}\right)=L\left(\mathscr{Z}_{\Pi^{\prime}} \cup\left(\bigcup_{\alpha^{\prime} \in \Pi^{\prime}} \mathscr{D}_{\Pi^{\prime}, \alpha^{\prime}}\right)\right)=L\left(\mathscr{Z}_{\Pi^{\prime}}\right) \cup\left(\bigcup_{\alpha^{\prime} \in \Pi^{\prime}} L\left(\mathscr{D}_{\Pi^{\prime}, \alpha^{\prime}}\right)\right) \\
& =\mathscr{Z}_{\Pi} \cup\left(\bigcup_{\alpha \in L\left(\Pi^{\prime}\right)} \mathscr{D}_{\Pi, \alpha}\right)=\mathscr{Z}_{\Pi} \cup\left(\bigcup_{\alpha \in \Pi} \mathscr{D}_{\Pi, \alpha}\right)
\end{aligned}
$$

using Proposition 3.6, as required to complete the proof of (d) in all cases.

The second inclusion in (e) follows from (d). For the first, note that its left hand side is contained in $\mathbb{R}_{\geq 0} \Pi \backslash\{0\}$. Hence it suffices to show that $\Phi_{+} \cap \mathscr{Z}=\emptyset$ and 
$\mathscr{Z} \cap \mathscr{Q}=\{0\}$. The former is clear since $\langle\alpha, \alpha\rangle=1$ for $\alpha \in \Phi$ while $\langle\alpha, \alpha\rangle \leq 0$ for $\alpha \in \mathscr{Z}$ by Proposition 3.2(c), while the latter holds by Lemma 9.11(b). Part (f) follows from (a) and (e).

9.13. We shall define below a subset $\mathscr{Z}_{\infty}$ of $\mathbb{R}_{>0} \Pi$ and a function $b: \mathscr{Z}_{\infty} \rightarrow C$ (which will turn out to coincide with $b$ in Theorem $9.9(\mathrm{a})$ ). To do this, fix $\gamma \in \mathbb{R}_{>0} \Pi$ and attempt to associate to $\gamma$ a sequence $b(\gamma)=\left(\beta_{n}\right)_{n \in \mathbb{N}} \in C$, using Lemma 9.11(b) as follows. Set $\gamma_{0}:=\gamma$. Then $\beta_{0}$ is defined if and only if $\gamma_{0} \in \mathbb{R}_{\geq 0} \Pi \backslash \mathscr{K}$, in which case $\beta_{0}$ is defined to be the unique element of $\Pi$ such that $\left\langle\beta_{0}, \gamma\right\rangle>0$. In general, for $m \in \mathbb{N}, \beta_{m+1}$ is defined if and only if $\beta_{0}, \ldots, \beta_{m}$ are all defined and, further, $\gamma_{m+1}:=s_{\beta_{m}} \cdots s_{\beta_{0}}(\gamma) \in \mathbb{R}_{\geq 0} \Pi \backslash \mathscr{K}$; then we define $\beta_{m+1}$ to be the unique element of $\Pi$ with $\left\langle\beta_{m+1}, \gamma_{m+1}\right\rangle>0$. Note $\beta_{m+1} \neq \beta_{m}$ when they are both defined. Define $\mathscr{Z}_{\infty}$ to be the set of all $\gamma \in \mathbb{R}_{\geq 0} \Pi$ such that $\beta_{n}$ is defined for all $n \in \mathbb{N}$, and define $b: \mathscr{Z}_{\infty} \rightarrow C$ by $b(\gamma):=\left(\beta_{n}\right)_{n \in \mathbb{N}}$ for any $\gamma \in \mathscr{Z}_{\infty}$. Note that $\mathscr{Z}_{\infty}$ is a possibly non-convex, blunt cone and $b$ is constant on $\mathbb{R}_{>0}$-orbits on $\mathscr{Z}_{\infty}$.

Lemma. (a) $b: \mathscr{Z}_{\infty} \rightarrow C$ is continuous.

(b) For $\beta=\left(\beta_{n}\right)_{n} \in C, b^{-1}(\beta)=\mathscr{Z}_{\infty} \cap \bigcap_{i \in \mathbb{N}} H_{\beta_{i}^{\prime}}^{>}$where $\beta_{i}^{\prime}:=s_{\beta_{0}} \cdots s_{\beta_{i-1}}\left(\beta_{i}\right)$.

Proof. To prove (a), it will suffice to show that for $n \in \mathbb{N}$ and $\beta_{0}, \ldots, \beta_{n} \in \Pi$ with $\beta_{i-1} \neq \beta_{i}$ for $i=1, \ldots, n$, the set $U:=\left\{\gamma \in \mathscr{Z}_{\infty} \mid(b(\gamma))_{i}=\beta_{i}\right.$ for $\left.i=1, \ldots, n\right\}$ is open in $\mathscr{Z}_{\infty}$. But from the definition of $b$, for any $\gamma \in \mathscr{Z}_{\infty}$, one has $\gamma \in U$ if and only if

$$
\left\langle\gamma, \beta_{0}\right\rangle>0,\left\langle s_{\beta_{0}} \gamma, \beta_{1}\right\rangle>0, \ldots,\left\langle s_{\beta_{n-1}} \cdots s_{\beta_{0}}(\gamma), \beta_{n}\right\rangle>0 .
$$

This implies that $U=\mathscr{Z}_{\infty} \cap \bigcap_{i=0}^{n} H_{\beta_{i}^{\prime}}^{>}$where $\beta_{i}^{\prime}=s_{\beta_{0}} \cdots s_{\beta_{i-1}}\left(\beta_{i}\right)$ for $i=0, \ldots, n$. This description shows $U$ is open in $\mathscr{Z}_{\infty}$, proving (a). A similar argument shows that for $\gamma \in \mathscr{Z}_{\infty}$ and $\beta=\left(\beta_{n}\right)_{n} \in C$, one has $b(\gamma)=\beta$ if and only if $\left\langle\gamma, \beta_{i}^{\prime}\right\rangle>0$ for all $i \in \mathbb{N}$, where $\beta_{i}^{\prime}$ is as in (b). This completes the proof of (b).

9.14. In order to describe and prove some further properties of the function $b$, it is convenient to partially order $V$ by $v_{1} \leq v_{2}$ if $v_{2}-v_{1} \in \mathbb{R}_{>_{0}} \Pi$, for $v_{1}, v_{2} \in V$. For $\gamma \in \mathbb{R}_{\geq 0} \Pi \backslash \mathscr{K}$ and $\beta \in \Pi$, we have $s_{\beta}(\gamma)<\gamma$ if $\langle\gamma, \beta\rangle>0$ and $s_{\beta}(\gamma)>\gamma$ if $\langle\gamma, \beta\rangle<0$, these being the only possibilities (any coarser partial order on $V$ with these properties could be used in place of $\leq$ below).

Lemma. Let $\gamma^{\prime} \in \mathscr{Z}_{\infty}, b\left(\gamma^{\prime}\right)=\beta^{\prime}=\left(\beta_{n}^{\prime}\right)_{n} \in C$ and $\alpha=\left(\alpha_{n}\right)_{n} \in C$ with $\alpha \neq \beta^{\prime}$. Let $m:=\max \left(\left\{n \in \mathbb{N} \mid \alpha_{j}=\beta_{j}^{\prime}\right.\right.$ for $\left.\left.j=0, \ldots, n-1\right\}\right) \in \mathbb{N}$. For any $n \in \mathbb{N}$, write $\gamma_{n}^{\prime}:=s_{\beta_{n-1}^{\prime}} \cdots s_{\beta_{0}^{\prime}}\left(\gamma^{\prime}\right) \in \mathbb{R}_{\geq 0} \Pi$ and set $\delta_{n}:=s_{\alpha_{n-1}} \cdots s_{\alpha_{0}}\left(\gamma^{\prime}\right)$.

(a) $\gamma_{0}^{\prime}>\gamma_{1}^{\prime}>\gamma_{2}^{\prime} \ldots$

(b) $\delta_{0}>\delta_{1}>\ldots>\delta_{m}$ and $\delta_{m}<\delta_{m+1}<\delta_{m+2}<\ldots$

(c) $\gamma_{n}^{\prime}=\delta_{n}$ for $n=0,1, \ldots, m$ and $\delta_{n}>\gamma_{n}^{\prime}$ for all $n \geq m+1$.

(d) $\mathscr{Z}_{\infty}=\overline{\mathscr{Z}} \backslash \mathscr{Z}$.

(e) $\lim _{n \rightarrow \infty} \gamma_{n}^{\prime}=0$ and $\gamma^{\prime} \in \mathscr{Q}$.

(f) $\left\langle\rho, \delta_{n}\right\rangle \rightarrow \infty$ as $n \rightarrow \infty$.

(g) If $z \in \mathscr{Z} \backslash\{0\}$ and $\left(w_{n}\right)_{n \in \mathbb{N}}$ is any sequence of pairwise distinct elements of $W$, then $\left\langle\rho, w_{n} z\right\rangle \rightarrow \infty$ as $n \rightarrow \infty$.

Proof. For $\tau \in \mathbb{R}_{\geq 0} \Pi \backslash \mathscr{K}$, there is a unique $\epsilon \in \Pi$ such that $\langle\tau, \epsilon\rangle>0$, by Lemma 9.11 and for any $\epsilon^{\prime} \in \Pi \backslash\{\epsilon\}$, one has $s_{\epsilon^{\prime}} \tau>\tau$ and $s_{\epsilon^{\prime}} \tau \in \mathbb{R}_{\geq 0} \Pi \backslash \mathscr{K}$. The claims in parts (a)-(c) on $\gamma_{n}^{\prime}, \delta_{n}$ follow easily from these observations by induction on $n$. 
Next we prove (d). For any $\gamma \in \overline{\mathscr{Z}} \backslash \mathscr{Z} \subseteq \mathbb{R}_{\geq 0} \Pi \backslash \mathscr{K}$, there is a unique $\beta \in \Pi$ with $\langle\beta, \gamma\rangle>0$ by Lemma 9.11(b); further, one has $s_{\beta}(\gamma) \in \overline{\mathscr{Z}} \backslash \mathscr{Z}$ since $\overline{\mathscr{Z}} \backslash \mathscr{Z}$ is $W$-invariant. It follows by induction on $n$ that for all $n \in \mathbb{N}$, the simple root $\beta_{n}$ in the construction of $b(\gamma)$ above is defined, so $b(\gamma)$ is defined and $\overline{\mathscr{Z}} \backslash \mathscr{Z} \subseteq \mathscr{Z}_{\infty}$. For the reverse inclusion, it will suffice by Theorem 5.1 (b) and definition of $\mathscr{Y}$ to show that $\gamma^{\prime} \notin \mathscr{Z}$ and $w\left(\gamma^{\prime}\right) \in \mathbb{R}_{\geq 0} \Pi$ for all $w \in W$ (since $\gamma^{\prime} \in \mathscr{Z}_{\infty}$ is arbitrary). Since $\beta_{i}^{\prime} \neq \beta_{i+1}^{\prime}$ for all $i$, one has $s_{\beta_{0}^{\prime}} \cdots s_{\beta_{k}^{\prime}}\left(\beta_{k+1}^{\prime}\right) \in \Phi_{+}$for all $k \in \mathbb{N}$, and these roots are pairwise distinct. Since $\left\langle\beta_{k+1}^{\prime}, \gamma_{k+1}^{\prime}\right\rangle>0$, we have $\left\langle s_{\beta_{0}^{\prime}} \cdots s_{\beta_{k}^{\prime}}\left(\beta_{k+1}^{\prime}\right), \gamma^{\prime}\right\rangle>0$ for all $k$. Lemma 1.10(a) implies that $\gamma^{\prime} \notin-\mathscr{X}$ and then Proposition 3.2(a) implies that $\gamma^{\prime} \notin \mathscr{Z}$. Now we let $w \in W$ and show $w \gamma^{\prime} \in \mathbb{R}_{\geq 0} \Pi$. By choosing $\alpha$ appropriately, we may assume without loss of generality that $w=s_{\alpha_{p-1}} \cdots s_{\alpha_{0}}$ where $p \in \mathbb{N}$. Then $w\left(\gamma^{\prime}\right)=\delta_{p} \geq \gamma_{p}^{\prime} \in \mathbb{R}_{\geq 0} \Pi$ by (a)-(c). By definition of $\leq$, we also have $w\left(\gamma^{\prime}\right)-\gamma_{p}^{\prime} \in \mathbb{R}_{\geq 0} \Pi$ so $w\left(\gamma^{\prime}\right) \in \mathbb{R}_{\geq 0} \Pi$ as required. This completes the proof of (d).

For (e), let $\lambda:=\inf \left(\left\{\left\langle\rho, w \gamma^{\prime}\right\rangle \mid w \in W\right\}\right) \in \mathbb{R}_{\geq 0}$. By (d) and Lemma 6.1. there exists $v \in \overline{W \gamma^{\prime}} \cap \mathscr{K}$ with $\rho(v)=\lambda$ and a sequence $\left(w_{n}\right)_{n \in \mathbb{N}}$ in $W$ such that $w_{n} \gamma^{\prime} \rightarrow v$. For each $n \in \mathbb{N}$, write $w_{n}=x_{n} y_{n}$, where $l\left(w_{n}\right)=l\left(x_{n}\right)+l\left(y_{n}\right)$, as follows. Take a reduced expression $w_{n}=s_{\epsilon_{q}} \ldots s_{\epsilon_{0}}$ (depending on $n$ ) with $\epsilon_{i} \in \Pi$, and set $p=\max \left(\left\{n \in \mathbb{N} \mid n \leq q+1, \epsilon_{i}=\gamma_{i}^{\prime}\right.\right.$ for $\left.\left.i=0, \ldots, n-1\right\}\right)$. Define $y_{n}=s_{\epsilon_{p-1}} \cdots s_{\epsilon_{0}}$ and $x_{n}=s_{\epsilon_{q}} \cdots s_{\epsilon_{p}}$. Also write $m_{n}:=l\left(y_{n}\right)$. By choosing $\alpha$ appropriately depending on $w_{n}$, (a)-(c) imply that $\gamma^{\prime} \geq y_{n} \gamma^{\prime}$ and $w_{n} \gamma^{\prime} \geq y_{n} \gamma^{\prime}$ for all $n$. Hence $\lambda \leq\left\langle\rho, y_{n} \gamma^{\prime}\right\rangle \leq\left\langle\rho, w_{n} \gamma^{\prime}\right\rangle$ for all $n$. As $n \rightarrow \infty,\left\langle\rho, w_{n} \gamma^{\prime}\right\rangle \rightarrow \lambda$ and so $\left\langle\rho, y_{n} \gamma^{\prime}\right\rangle \rightarrow \lambda$ also. But $w_{n} \gamma^{\prime}-y_{n} \gamma^{\prime} \in \mathbb{R}_{\geq 0} \Pi$ and $\left\langle w_{n} \gamma^{\prime}-y_{n} \gamma^{\prime}, \rho\right\rangle \rightarrow 0$ as $n \rightarrow \infty$ imply that $w_{n} \gamma^{\prime}-y_{n} \gamma^{\prime} \rightarrow 0$ also. Since $w_{n} \gamma^{\prime} \rightarrow v$, we get $y_{n} \gamma^{\prime} \rightarrow v$ as well. But $y_{n} \gamma^{\prime}=\gamma_{m_{n}}^{\prime}$. Since the sequence $\left(\left\langle\rho, \gamma_{n}^{\prime}\right\rangle\right)_{n}$ in $\mathbb{R}_{\geq 0}$ is strictly monotonic decreasing and $\left\langle\rho, \gamma_{m_{n}}^{\prime}\right\rangle \rightarrow \lambda$ as $n \rightarrow \infty$, we must have, by definition of $\lambda, m_{n} \rightarrow \infty$ and $\left\langle\rho, \gamma_{n}^{\prime}\right\rangle \rightarrow \lambda$ as $n \rightarrow \infty$. Now for any $k$ with $m_{k}>n$, we have $\gamma_{n}^{\prime}-\gamma_{m_{k}}^{\prime} \in \mathbb{R}_{\geq 0} \Pi$. Letting $k \rightarrow \infty$ gives $\gamma_{n}^{\prime}-v \in \mathbb{R}_{\geq 0} \Pi$. Since $\left\langle\gamma_{n}^{\prime}-v, \rho\right\rangle \rightarrow \lambda-\lambda=0$ as $n \rightarrow \infty$, we get $\gamma_{n}^{\prime} \rightarrow v$ as $n \rightarrow \infty$. Hence $\lim _{n \rightarrow \infty} \gamma_{n}^{\prime}=v \in \mathscr{K}$.

We show next that $v=0$. Suppose to the contrary that $v \neq 0$. Then $\left\langle\rho, \gamma_{n}^{\prime}\right\rangle \geq$ $\lambda>0$ for all $n \in \mathbb{N}$. Since $\gamma^{\prime}$ is in the $W$-invariant set $\overline{\mathscr{Z}} \backslash \mathscr{Z}$, and $\gamma_{n}^{\prime} \in W \gamma$, we get $\gamma_{n}^{\prime} \in \overline{\mathscr{Z}} \backslash \mathscr{Z} \subseteq \cup_{\alpha \in \Pi} \mathscr{D}_{\alpha}$. Let $\gamma_{n}^{\prime} \in \mathscr{D}_{\tau_{n}}$, where $\tau_{n} \in \Pi$. By Lemma 9.12(a)(b), $\left\langle\gamma_{n}^{\prime}, \tau_{n}^{\vee}\right\rangle \geq k_{\rho}\left\langle\rho, \gamma_{n}^{\prime}\right\rangle \geq \lambda k_{\rho}>0$ and similarly $\left\langle\gamma_{n}^{\prime}, \sigma^{\vee}\right\rangle \leq-\lambda k_{\rho}<0$ for $\sigma \in \Pi \backslash\left\{\tau_{n}\right\}$. Since $\gamma_{n}^{\prime} \rightarrow v$ as $n \rightarrow \infty$, there is some $\tau \in \Pi$ such that $\tau_{n}=\tau$ for all sufficiently large $n$, and $v \in \mathscr{D}_{\tau}$. But then $\left\langle v, \tau^{\vee}\right\rangle \geq k_{\rho}\langle\rho, v\rangle>0$ contrary to $v \in \mathscr{K}$. This shows that $v=0$. Now $w_{n} \gamma^{\prime} \rightarrow v$ and $\left\langle\gamma^{\prime}, \gamma^{\prime}\right\rangle=\left\langle w_{n} \gamma^{\prime}, w_{n} \gamma^{\prime}\right\rangle \rightarrow$ $\langle v, v\rangle=0$ as $n \rightarrow \infty$ implies that $\gamma^{\prime}$ is isotropic. This completes the proof of (e).

Now we prove (f). For any $l \in \mathbb{N}, \delta_{l+1}=s_{\alpha_{l}}\left(\delta_{l}\right)=\delta_{l}-\left\langle\delta_{l}, \alpha_{l}^{\vee}\right\rangle \alpha_{l}$. Assume $l \geq m$. Then $\left\langle\delta_{l}, \alpha_{l}^{\vee}\right\rangle\left\langle 0\right.$ since $\delta_{l+l}>\delta_{l}$, so $-\left\langle\delta_{l}, \alpha_{l}^{\vee}\right\rangle \geq k_{\rho}\left\langle\rho, \delta_{l}\right\rangle \geq k_{\rho}\left\langle\rho, \delta_{m}\right\rangle$ where $k_{\rho}$ is as in Lemma 9.12, using $\delta_{l}-\delta_{m} \in \mathbb{R}_{\geq 0} \Pi$ for the last inequality. Hence

$$
\left\langle\rho, \delta_{l+1}-\delta_{l}\right\rangle=-\left\langle\delta_{l}, \alpha_{l}^{\vee}\right\rangle\left\langle\rho, \alpha_{l}\right\rangle \geq k_{\rho}\left\langle\rho, \delta_{m}\right\rangle\left\langle\rho, \alpha_{l}\right\rangle \geq \epsilon_{\rho} k_{\rho}\left\langle\rho, \delta_{m}\right\rangle .
$$

Setting $K:=\epsilon_{\rho} k_{\rho}\left\langle\rho, \delta_{m}\right\rangle>0$, we get $\left\langle\rho, \delta_{N+m}\right\rangle \geq\left\langle\rho, \delta_{m}\right\rangle+N K$ for all $N \in \mathbb{N}$ which implies (f) (the above estimates suffice for purposes here but are obviously far from sharp).

For the proof of (g), we may assume without loss of generality that $z \in \mathscr{K} \backslash\{0\}$. Hence $v:=-z \in \mathscr{C}$. Fix $N \in \mathbb{R}_{\geq 0}$. By 4.3 (a), there are only finitely many $x \in W z$ with $\langle v-x, \rho\rangle \leq N$. By Lemma 1.10 and Lemma 9.11(e), $\operatorname{Stab}_{W}(v)$ is a standard parabolic subgroup of $W$ of rank 0 or 1 , so it is finite. Hence for each $x \in W z$ with 
$\langle v-x, \rho\rangle \leq N$, there are only finitely many $w \in W v$ with $w v=x$. This implies that there are only finitely many $w \in W$ with $\langle v-w v, \rho\rangle \leq N$ i.e. $\langle w z, \rho\rangle \leq N-\langle v, \rho\rangle$. Since $N \geq 0$ is arbitrary, this establishes (g).

9.15. This section will establish that the fibers of $b$ are all non-empty, which is an important point in the proof of Theorem 9.9 . Though not strictly necessary for purposes here, we establish a stronger fact involving the dominance order $\preceq$ of $\Phi$, which is the partial order such that $\alpha \preceq \beta$ if for all $w \in W$ with $w(\beta) \in \Phi_{-}$, one has $w(\alpha) \in \Phi_{-}$(see [6], 2] or [31). If $\alpha, \beta \in \Phi_{+}$, one has $\alpha \preceq \beta$ if and only if $\langle\alpha, \beta\rangle \geq 1$ and $l\left(s_{\alpha}\right) \leq l\left(s_{\beta}\right)$. The above two characterizations of dominance order apply to arbitrary based root systems. For universal root systems (generic or not), the second criterion immediately implies the following fact: for $\alpha, \beta \in \Phi_{+}$, one has $\alpha \preceq \beta$ if and only if, writing (in the unique possible way) $\beta=s_{\beta_{1}} \ldots s_{\beta_{n-1}}\left(\beta_{n}\right)$ where all $\beta_{i} \in \Pi$ and $l\left(s_{\beta}\right)=2 n-1$, one has $\alpha=s_{\beta_{1}} \ldots s_{\beta_{m-1}}\left(\beta_{m}\right)$ for some $1 \leq m \leq n$.

Recall also the definitions from $\$ 5$ of the set $R_{+}$of rays spanned by positive roots and the set $R_{0}=\operatorname{Acc}\left(R_{+}\right)$of its limit rays in ray $(V)$. Note that, since limit rays of the set of root rays are isotropic, $\left(\bigcup R_{0}\right) \backslash\{0\} \subseteq \overline{\mathscr{Z}} \backslash \mathscr{Z}=\mathscr{Z}_{\infty}$ by Lemmas 9.11 and 9.14. Since $b: \mathscr{Z}_{\infty} \rightarrow C$ has blunt, possibly non-convex cones as its fibers, it determines a partition $R_{0}=\dot{\cup}_{\beta \in C}\left(R_{0} \cap \operatorname{ray}\left(b^{-1}(\beta) \cup\{0\}\right)\right.$ ) (except it is not yet clear that all the sets on the right are non-empty). The following proposition gives a condition for a sequence of positive root rays to have all its limit rays in one set in this partition, and makes it clear that these sets are all non-empty.

Proposition. Fix $\beta=\left(\beta_{n}\right)_{n \in \mathbb{N}} \in C$. Set $\beta_{n}^{\prime}:=s_{\beta_{0}} \cdots s_{\beta_{n-1}}\left(\beta_{n}\right) \in \Phi_{+}$.

(a) Let $\alpha_{n} \in \Phi_{+}$for all $n \in \mathbb{N}$. Then $\operatorname{Acc}\left(\left(\mathbb{R}_{\geq 0} \alpha_{n}\right)_{n}\right) \subseteq R_{0} \cap \operatorname{ray}\left(b^{-1}(\beta) \cup\{0\}\right)$ if and only if for all $m \in \mathbb{N}$, one has $\beta_{m}^{\prime} \preceq \alpha_{n}$ for almost all $n \in \mathbb{N}$.

(b) $\operatorname{Acc}\left(\left(\mathbb{R}_{\geq 0} \beta_{n}^{\prime}\right)_{n \in \mathbb{N}}\right) \subseteq \operatorname{ray}\left(b^{-1}(\beta) \cup\{0\}\right)$. Hence $b$ is surjective.

Proof. It is sufficient to prove (a) under the extra assumption that $\left(\mathbb{R}_{\geq 0} \alpha_{n}\right)_{n}$ has a unique limit ray $\mathbb{R}_{\geq 0} \alpha$ i.e. $\left\{\mathbb{R}_{\geq 0} \alpha\right\}=\operatorname{Acc}\left(\left(\mathbb{R}_{\geq 0} \alpha_{n}\right)_{n}\right)$ or equivalently, $\widehat{\alpha}=$ $\lim _{n \rightarrow \infty} \widehat{\alpha}_{n}$. First consider the case that $\alpha$ is non-isotropic. Without loss of generality, assume $\alpha \in \Phi_{+}$. Then $\alpha_{n}=\alpha$ for almost all $n$. Moreover $\mathbb{R}_{\geq 0} \alpha \notin R_{0}$ and, since each positive root dominates only finitely many positive roots and the $\beta_{i}$ are pairwise distinct, there is some $m \in \mathbb{N}$ such that $\beta_{m} \leq \alpha_{n}$ for no $n \in \mathbb{N}$. Hence (a) holds if $\alpha$ is non-isotropic. Now assume that $\alpha$ is isotropic. Then, since $\mathbb{R}_{\geq 0} \alpha$ is a limit ray of the set of rays spanned by positive roots, one has $\alpha \in \mathscr{Z}_{\infty}$, as previously noted, so $b(\alpha)$ is defined. In this case, we have $\mathbb{R}_{>0} \alpha \in R_{0} \cap \operatorname{ray}\left(b^{-1}(\beta) \cup\{0\}\right)$ if and only if $b(\alpha)=\beta$. In the notation of Lemma 9.13(b), we have $b(\alpha)=\left(\beta_{n}\right)_{n}$ if and only if for all $m \in \mathbb{N},\left\langle\alpha, \beta_{m}^{\prime}\right\rangle>0$.

We claim that this holds if and only if for all $m,\left\langle\alpha_{n}, \beta_{m}^{\prime}\right\rangle>0$ for almost all $n$. Fix $m$. Since $\widehat{\alpha}_{n} \rightarrow \widehat{\alpha}$, it is clear that $\left\langle\alpha, \beta_{m}^{\prime}\right\rangle>0$ implies $\left\langle\alpha_{n}, \beta_{m}^{\prime}\right\rangle>0$ for almost all $n$. Conversely, suppose that $\left\langle\alpha_{n}, \beta_{m}^{\prime}\right\rangle>0$ for almost all $n$. Since $\widehat{\alpha}_{n} \rightarrow \widehat{\alpha}$, it is clear that $\left\langle\widehat{\alpha}, \beta_{m}^{\prime}\right\rangle \geq 0$ and we have to show equality cannot occur. Write $w:=s_{\beta_{m-1}} \ldots s_{\beta_{0}}$. We have to show that $\left\langle\widehat{\alpha}, w^{-1} \beta_{m}\right\rangle \neq 0$ i.e. $\left\langle w \widehat{\alpha}, \beta_{m}\right\rangle \neq 0$. But $0 \neq \widehat{\alpha} \in \overline{\mathscr{Z}} \cap \mathscr{Q}$ and hence $0 \neq w \widehat{\alpha} \in \overline{\mathscr{Z}} \cap \mathscr{Q} \subseteq \mathbb{R}_{\geq_{0}} \Pi \cap \mathscr{Q}$. By Lemma 9.12 . $\left|\left\langle w \widehat{\alpha}, \beta_{m}^{\vee}\right\rangle\right| \geq k_{\rho}\langle\rho, w \widehat{\alpha}\rangle>0$. This proves the claim.

Thus, $b(\alpha)=\beta$ if and only if for all $m,\left\langle\alpha_{n}, \beta_{m}^{\prime}\right\rangle>0$ for almost all $n$. Equivalently since $\Phi$ is universal, $b(\alpha)=\beta$ if and only if for all $m,\left\langle\alpha_{n}, \beta_{m}^{\prime}\right\rangle \geq 1$ for almost all $n$. Since $l\left(s_{\alpha_{n}}\right) \rightarrow \infty$ as $n \rightarrow \infty$, this is equivalent to the condition that 
for all $m, \beta_{m}^{\prime} \preceq \alpha_{n}$ for almost all $n$. This proves (a). Part (b) follows from (a) since $\beta_{0}^{\prime} \prec \beta_{1}^{\prime} \prec \beta_{2}^{\prime} \prec \ldots$ (and any sequence of distinct root rays has at least one limit ray).

9.16. Proof of Theorem 9.9. The first part of 9.9 (a) is already established as Lemma 9.11(c). The second part of (a) follows from Lemma 9.14(d)-(e), since $\gamma^{\prime} \in \mathscr{Z}_{\infty}$ there is arbitrary.

Let $\beta=\left(\beta_{n}\right)_{n} \in C$ and $w_{n}:=s_{\beta_{n-1}} \ldots s_{\beta_{0}} \in W$. Then

$$
b^{-1}(\beta) \cup\{0\} \subseteq\left\{z \in \overline{\mathscr{Z}} \mid \lim _{n \rightarrow \infty} w_{n} z=0\right\}
$$

by Lemma 9.14(e). Also,

$$
\overline{\mathscr{Z}} \backslash\left(b^{-1}(\beta) \cup\{0\}\right) \subseteq\left\{z \in \overline{\mathscr{Z}} \mid \lim _{n \rightarrow \infty}\left\langle\rho, w_{n} z\right\rangle=\infty\right\}
$$

To see this, note that $\mathscr{Z} \backslash\{0\}$ is contained in the right hand side by Lemma 9.14(g), and any point of $\overline{\mathscr{Z}} \backslash\left(\mathscr{Z} \cup b^{-1}(\beta)\right)$ is in $b^{-1}\left(\beta^{\prime}\right)$ for some $\beta^{\prime} \neq \beta$ in $C$ and so is in the right hand side by using Lemma 9.14(f). Now the left hand sides of 9.16.1)9.16.2 form a partition of $\overline{\mathscr{Z}}$ and the right hand sides are disjoint subsets of $\mathscr{Z}$. Hence the inclusions in 9.16.1 - 9.16.2 are actually equalities. By definition of $F_{\beta}$, this proves that $b^{-1}(\beta) \cup\{0\}=F_{\beta}$ and that Theorem 9.9(b) holds. It also implies that the sets $F_{\beta} \backslash\{0\}$ for $\beta \in C$ form a partition of $\mathscr{Z} \backslash \mathscr{Z}$, which proves Theorem 9.9(c) and that the function $b$ so defined coincides with the function $b$ defined in 9.13 .

Note that for $\beta \in C, F_{\beta}$ is a totally isotropic face of $\overline{\mathscr{Z}}$ by Lemma 7.10 (c) and $F_{\beta} \cap \mathscr{Z}=\{0\}$ by (a). Hence $b^{-1}(\beta)=F_{\beta} \backslash\{0\}$ is a blunt cone, which is non-empty by Proposition $9.15(\mathrm{~b})$. So $b^{-1}(\beta)$ is non-empty and connected. Since $C$ is totally disconnected, its connected components are its singleton subsets and therefore $\beta^{-1}(\beta)$ is a connected component of the domain $\overline{\mathscr{Z}} \backslash \mathscr{Z}$ of $b$. This proves Theorem $9.9(\mathrm{~d})$.

Next we prove Theorem 9.9 (e). Suppose $\beta \in C$. Let $F$ be any face of $\overline{\mathscr{Z}}$ such that $F \cap \mathscr{Z}=\{0\}$ and $F \supseteq F_{\beta}$. Then $F \backslash\{0\}$ is a connected subset of $\overline{\mathscr{Z}} \backslash \mathscr{Z}$ and it contains the component $F_{\beta} \backslash\{0\}$, so by (b), $F \backslash\{0\}=F_{\beta} \backslash\{0\}$ and $F=F_{\beta}$. On the other hand, let $G$ be any face of $\overline{\mathscr{Z}}$ with the property $G \cap \mathscr{Z}=\{0\}$. If $G=\{0\}$, then $G \subseteq F_{\beta}$ for all $\beta \in C$. Otherwise, $G \backslash\{0\}$ is a non-empty connected subset of $\overline{\mathscr{Z}} \backslash \mathscr{Z}$ and is therefore contained in $F_{\beta} \backslash\{0\}$ for a unique $\beta \in C$. This completes the proof of (e).

Before the proof of Theorem 9.9(f), we recall relevant generalities about quotient maps. A map $q: X \rightarrow Y$ of topological spaces is said to be a quotient map if it is surjective and a set $U$ is open in $Y$ if and only if $q^{-1}(U)$ is open in $X$. In that case, any continuous map $f: X \rightarrow Z$ which is constant on each fiber $q^{-1}(y)$, for $y \in Y$, factors uniquely as a composite $f=f^{\prime} q$ where $f^{\prime}: Y \rightarrow Z$ is continuous. Any continuous, open (resp., closed), surjective map $q: X \rightarrow Y$ is a quotient map and then given any subset $Y^{\prime}$ of $Y$, the restriction of $q$ to a map $q^{\prime}: X^{\prime} \rightarrow Y$ is also an open (resp., closed) quotient map where $X^{\prime}:=q^{-1}\left(Y^{\prime}\right)$.

Consider the space $\operatorname{ray}(V)$. For the part of the argument involving $p$, it is convenient to identify ray $(V)$ with the unit sphere $\mathbb{S}$ in $V$ (with respect to some norm \|\| on $V$ induced by a positive definite inner product on $V$ ) by the homeomorphism $\mathbb{R}_{\geq 0} \alpha \mapsto \frac{1}{\|\alpha\|} \alpha$ for $0 \neq \alpha \in V$. The natural continuous map $\alpha \mapsto \mathbb{R}_{\geq 0} \alpha: V \backslash\{0\} \rightarrow$ $\operatorname{ray}(V)$ then identifies with the map $r: V \backslash\{0\} \rightarrow \mathbb{S}$ given by $\alpha \mapsto \frac{1}{\|\alpha\|} \alpha$. It is an 
open quotient map (in fact, it maps open balls in $V \backslash\{0\}$ to open balls in $\mathbb{S}$, where the balls in $\mathbb{S}$ are defined with respect to the metric obtained by restriction of the metric on $V$ induced by \|\|$)$. It follows that for any subset $X$ of $V$ which is a union of rays, the natural map $X \backslash\{0\} \rightarrow \operatorname{ray}(X \cup\{0\})$ given by restriction of $r$ is an open quotient map, where $X \backslash\{0\}$ (resp., ray $(X)$ ) is topologized as a subspace of $V(\operatorname{resp} ., \operatorname{ray}(V))$.

This applies in particular to the set $X=\overline{\mathscr{Z}} \cap \mathscr{Q}=\mathscr{Z}_{\infty} \cup\{0\}=(\overline{\mathscr{Z}} \backslash \mathscr{Z}) \cup\{0\}$. It follows that $b$ factors as $b=q p$ where $p, q$ are as in 9.9 (f) except that $q$ is so far only known to be continuous. To show $q$ is a closed quotient map, recall the identification (used in the proof of Proposition 5.3 ) of ray $\left(\mathbb{R}_{\geq 0} \Pi\right)$ with the polytope $P=\operatorname{conv}(\widehat{\Pi})$, where $\widehat{\Pi}:=\{\widehat{\alpha} \mid \alpha \in \Pi\}$, in the affine hyperplane $H=\{v \in V \mid$ $\langle\rho, v\rangle=1\}$. The identification is given by $\mathbb{R}_{\geq 0} \alpha \mapsto \widehat{\alpha}$ for $0 \neq \alpha \in \mathbb{R}_{\geq 0} \Pi$. Under this identification, $\operatorname{ray}(\mathscr{Q} \cap \overline{\mathscr{Z}})$ identifies with the closed, hence compact, subset $\left\{\widehat{\alpha} \mid \alpha \in \mathscr{Z}_{\infty}\right\}=\mathscr{Q} \cap \overline{\mathscr{Z}} \cap H$ of $P$. Since $q$ is a continuous map between compact Hausdorff spaces, it is closed. Since $b$ is surjective, so is $q$ and therefore $q$ is a quotient map as claimed. As it is a composite of quotient maps, $b$ is a quotient map too.

9.17. Retain the identification $\operatorname{ray}(\mathscr{Q} \cap \overline{\mathscr{Z}}) \subseteq \operatorname{ray}\left(\mathbb{R}_{\geq 0} \Pi\right)=P$ from the end of the preceding proof. (One can naturally identify $\operatorname{ray}(\bar{V})$ itself with the boundary of any convex polytope $P^{\prime}$ with $P$ as a face and 0 as interior point, if desired.) The identification induces an identification of $\operatorname{ray}(\overline{\mathscr{Z}})$ with a convex subset $Z$ of $P$ and $\operatorname{ray}(\overline{\mathscr{Z}})$ with the compact convex set $\bar{Z}$. Let $Q:=\operatorname{ray}(\mathscr{Q})$. The fibers of $q$ are precisely the maximal isotropic faces (isotropic in the sense of being contained in $Q$ ) of $\bar{Z}$ and they are also the connected components of $\bar{Z} \cap Q$. Further, $C$ is a quotient space of $\bar{Z} \cap Q$, identifying each connected component to a point. For arbitrary generic universal based root systems, we leave the determination of the dimensions of these connected components, description of their faces and face lattices etc as an open problem. If however $\Phi$ is in addition assumed to be weakly hyperbolic, the connected components of $\bar{Z} \cap Q$ reduce to points se questions can be answered easily (see 21] for some properties of weakly hyperbolic root systems in general).

Corollary. Suppose that $\Phi$ is weakly hyperbolic and generic universal.

(a) Let $\beta=\left(\beta_{n}\right)_{n \in \mathbb{N}} \in C$. Set $\beta_{n}^{\prime}:=s_{\beta_{0}} \cdots s_{\beta_{n-1}}\left(\beta_{n}\right) \in \Phi_{+}$. Then the limit $\lim _{n \rightarrow \infty} \mathbb{R}_{\geq 0} \beta_{n}^{\prime}=\mathbb{R}_{\geq 0} z_{\beta}$ exists in $\operatorname{ray}(V)$ and $\operatorname{ray}\left(b^{-1}(\beta) \cup\{0\}\right)=\left\{\mathbb{R}_{\geq 0} z_{\beta}\right\}$.

(b) $R_{0}=\operatorname{ray}(\mathscr{Q} \cap \overline{\mathscr{Z}})$.

(c) The $\operatorname{map} q: R_{0} \rightarrow C$ is a homeomorphism.

Remarks. It is easily checked that a generic universal root system is weakly hyperbolic if it has rank three, or if it has rank at least four and all inner products of distinct simple roots are (sufficiently close to) equal.

Proof. The isotropic face $F_{\beta}$ is one-dimensional (i.e. it is a ray) by the discussion in 9.2. Hence $\operatorname{ray}\left(F_{\beta}\right)=\left\{\mathbb{R}_{\geq 0} z_{\beta}\right\}$ for any non-zero $z_{\beta} \in F_{\beta}$. The sequence $\mathbb{R}_{\geq 0} \beta_{n}$ has at least one limit point, and any such limit point must be in $\operatorname{ray}\left(F_{\beta}\right)$ by Proposition 9.16. Part (a) follows. One has $R_{0} \subseteq \operatorname{ray}(\mathscr{Q} \cap \overline{\mathscr{Z}})$ in general and (a) implies the reverse inclusion in (b). Finally, (a) implies that the fibers of the quotient map $q$ are singletons, so (c) follows from (b). 
9.18. The proofs of the above results about generic universal root systems use a number of ingredients which may be shown to have at least partial analogues for general root systems, but the situation in general is far more complex. The following example illustrates some of the subtleties which occur.

Example. Suppose $(\Phi, \Pi)$ is such that $\Pi=\{\alpha, \beta, \gamma, \delta, \epsilon\}$ is linearly independent and that $\langle\alpha, \beta\rangle$ and $\langle\delta, \epsilon\rangle$ are less than -1 while $\langle\beta, \gamma\rangle,\langle\alpha, \gamma\rangle,\langle\gamma, \delta\rangle$ and $\langle\gamma, \epsilon\rangle$ are negative and the pairings of all other pairs of distinct roots are equal to zero. Write $\{\alpha, \beta, \delta, \epsilon\}=\Pi_{I}$ where $I \subseteq S$. Since $\Pi_{I}$ has two components $\Pi_{J}:=\{\alpha, \beta\}$ and $\Pi_{K}:=\{\gamma, \delta\}$, our general results imply that $\mathscr{Z} \cap \mathbb{R} \Pi_{I}=\mathscr{Z}_{I}=\mathscr{Z}_{J}+\mathscr{Z}_{K}$ and similarly $\overline{\mathscr{Z}} \cap \mathbb{R}_{I}=\overline{\mathscr{Z}}_{I}=\overline{\mathscr{Z}}_{J}+\overline{\mathscr{Z}}_{K}$.

Note $\left(\Phi_{I}, \Pi_{J}\right),\left(\Phi_{K}, \Pi_{K}\right)$ are (dihedral) generic universal; use for them the notation introduced previously for such systems. Then $\mathscr{Z}_{J}=\{0\} \cup \mathbb{R}_{>0}\{u(\alpha, \beta), u(\beta, \alpha)\}$ and $\overline{\mathscr{Z}}_{J}=\mathbb{R}_{\geq 0}\{u(\alpha, \beta), u(\beta, \alpha)\}$. So $\overline{\mathscr{Z}}_{I}=\mathbb{R}_{\geq 0}\{u(\alpha, \beta), u(\beta, \alpha), u(\delta, \epsilon), u(\epsilon, \delta)\}$, which is a four-dimensional simplicial cone, while

$$
\begin{aligned}
\mathscr{Z}_{I}=\mathbb{R}_{>0}\{u(\alpha, \beta), u(\beta, \alpha) & , u(\delta, \epsilon), u(\epsilon, \delta)\} \\
& \dot{\cup} \mathbb{R}_{>0}\{u(\alpha, \beta), u(\beta, \alpha)\} \cup \dot{\cup} \mathbb{R}_{>0}\{u(\delta, \epsilon), u(\epsilon, \delta)\} \cup \dot{\cup}\{0\}
\end{aligned}
$$

is the union of the relative interior of $\overline{\mathscr{Z}}_{I}$, the relative interiors of two opposite two-dimensional faces of $\overline{\mathscr{Z}}_{I}$ and $\{0\}$. Note that $\overline{\mathscr{Z}}_{I} \cap \mathscr{Q}$ is the union of the four two-dimensional faces of $\overline{\mathscr{Z}}_{I}$ whose relative interiors are not contained in $\mathscr{Z}_{I}$. Hence the relative interiors of the facets of $\overline{\mathscr{Z}}_{I}$ consist of non-isotropic points; these relative interiors are contained in $\overline{\mathscr{Z}}$ but not in $\mathscr{Z}$, so $\overline{\mathscr{Z}} \nsubseteq \mathscr{Z} \cup \mathscr{Q}$ even though $W$ is irreducible. The facets of $\overline{\mathscr{Z}}_{I}$ are faces of $\overline{\mathscr{Z}}$; they are neither closures of faces of $\mathscr{Z}$, nor totally isotropic.

The set of limit rays of positive root rays from $\Phi_{I}$ is

$$
R_{W_{I}, 0}=\left\{\mathbb{R}_{\geq 0} u(\alpha, \beta), \mathbb{R}_{\geq 0} u(\beta, \alpha), \mathbb{R}_{\geq 0} u(\delta, \epsilon), \mathbb{R}_{\geq 0} u(\epsilon, \delta)\right\} .
$$

This is properly contained in $\overline{\mathscr{Z}}_{I} \cap \mathscr{Q}$. On the other hand, computations like those in Example 7.12 show that any ray in $\overline{\mathscr{Z}}_{I} \cap \mathscr{Q}$ is an element of $R_{0}$.

For generic choices of the inner products as above, the form $\langle-,-\rangle$ on $\mathbb{R} \Pi$ has Witt index 2 and the four above-mentioned two-dimensional faces of $\overline{\mathscr{Z}}_{I}$ are therefore maximal totally isotropic faces of $\overline{\mathscr{Z}}$. Any one of the four extreme rays of $\overline{\mathscr{Z}}_{I}$ is equal to an intersection of two of these faces, so not all pairs of distinct faces from amongst these four maximal isotropic faces of $\overline{\mathscr{Z}}$ intersect in $\{0\}$.

In particular, the example shows that the restriction to generic points in Question 9.6 (a), (e) is necessary in general (it is not necessary in the generic universal case) and that the restriction to irreducible $\Phi$ is necessary in Question 9.6(b).

\section{Facial structure of the imaginary CONE}

10.1. Recall from 2.8 the notion of facial closure of a subset of $W$. The following lemma collects properties of facial closures under the assumptions 4.1(i)-(iii).

Lemma. (a) A standard facial subgroup of a standard facial subgroup of $W$ is a standard facial subgroup of $W$.

(b) A facial subgroup of a facial subgroup of $W$ is a facial subgroup of $W$.

(c) Any intersection of facial subgroups of $W$ is facial. 
(d) The facial closure of any $X \subseteq W$ exists. It is equal to the intersection of all facial subgroups containing $X$. It is also the unique facial subgroup of $W$ containing $X$ and of minimal rank amongst such facial subgroups.

(e) Let $W_{i}$ for $i \in I$ be reflection subgroups of $W$, all with no finite type component. Then the facial closure $W^{\prime}$ of $\cup_{i} W_{i}$ has no finite type components.

Proof. Part (a) follows from 4.2(f). For (b), let $W_{2}$ be facial in $W_{1}$ where $W_{1}$ is facial in $W$. Say $W_{1}=y W_{1}^{\prime} y^{-1}$ where $y \in W$ and $W_{1}^{\prime}$ is standard facial in $W$. Then $y^{-1} W_{2} y$ is facial in $y^{-1} W_{1} y=W_{1}^{\prime}$, say $y^{-1} W_{2} y=x W_{2}^{\prime} x^{-1}$ where $W_{2}^{\prime}$ is standard facial in $W_{1}^{\prime}$. Then $W_{2}=y x W_{2}^{\prime}(y x)^{-1}$ where $y x \in W$ and $W_{2}^{\prime}$ is standard facial in $W$ by (a). This proves (b). Parts (c)-(d) follow from the discussion in 2.8, since $W$ is assumed here to be of finite rank.

For (e), note first that $W^{\prime}$ is finitely generated since it is parabolic in $W$. Let $W^{\prime \prime} \subseteq W^{\prime}$ be the reflection subgroup of $W^{\prime}$ such that $\Pi_{W^{\prime \prime}}$ is equal to the union of the infinite type components of $\Pi_{W^{\prime}}$. By 8.5 (c), $W^{\prime \prime}$ is facial in $W$. Now any infinite type component of $\Phi_{W_{i}}$ is contained in some infinite type component of $\Phi_{W^{\prime \prime}}$ and hence it is contained in one of the irreducible components of $\Phi_{W^{\prime \prime}}$. It follows that $\Phi_{W_{i}} \subseteq \Phi_{W^{\prime \prime}}$ for all $i$ and hence that $W_{i} \subseteq W^{\prime \prime}$. Since $W^{\prime \prime}$ is facial, we get $W^{\prime} \subseteq W^{\prime \prime}$ by definition of facial closure, hence $W^{\prime \prime}=W^{\prime}$.

10.2. The above implies that the set of facial subgroups of $W$, ordered by inclusion, is a complete lattice. We now define a certain subposet $\mathscr{W}$ of this lattice, which plays an important role in this section. The subposet is itself a complete lattice with join given by restriction of the join in the family of facial subgroups.

Let $\mathscr{W}=\left\{w W_{I} w^{-1} \mid w \in W, I \in F_{\text {inf }}\right\}$ denote the set of all special facial subgroups of $W$ i.e facial subgroups with no finite irreducible components. We always consider $\mathscr{W}$ with partial order given by inclusion. For any parabolic subgroups $W^{\prime} \subsetneq W^{\prime \prime}$ of $W$, the rank of $W^{\prime}$ is strictly less than that of $W^{\prime \prime}$. It follows than any non-empty subset of $\mathscr{W}$ has at least one minimal element and at least one maximal element, and any chain $W_{0} \subsetneq \ldots \subsetneq W_{n}$ in $\mathscr{W}$ has length $n$ bounded by the rank of $W$.

Proposition. (a) The family $\mathscr{W}$ of subgroups of $W$ forms a complete lattice.

(b) The join of a subset of $\mathscr{W}$ is the facial closure of its union.

(c) The meet of a family $W_{i}$ for a $I$ in any index set $I$ is $W^{\prime}$, where $W^{\prime} \in \mathscr{W}$ is such that $\Pi_{W^{\prime}}$ is the union of all infinite type components of $\Pi_{\cap_{i} W_{i}}$.

Proof. Let $W_{i}$ for $i \in I$ be a family of elements of $\mathscr{W}$. By Lemma 10.1(e), the facial closure $W^{\prime}$ of $\cup_{i} W_{i}$ is a facial subgroup which has no infinite type components; clearly, $W^{\prime}$ is the minimum element of $\mathscr{W}$ containing all $W_{i}$. This proves that $\mathscr{W}$ is a complete lattice with join as in (b). Now we consider the meet of the family $\left\{W_{i}\right\}_{i \in I}$. Note that $W^{\prime}$ as defined in (c) is in $\mathscr{W}$ by Lemma 10.1(c) and 8.5(c). Clearly, $W^{\prime} \subseteq W_{i}$ for all $i$. If $W^{\prime \prime} \in \mathscr{W}$ with $W^{\prime \prime} \subseteq W_{i}$ for all $i$, then $W^{\prime \prime} \subseteq \cap_{i} W_{I}$. Here, $\cap_{i} W_{i}$ is a facial subgroup. Hence each (necessarily infinite type) component of $\Phi_{W^{\prime \prime}}$ is contained in some (infinite type) component of $\Phi_{\cap_{i} W_{I}}$, and hence it is contained in $\Phi_{W^{\prime}}$. This implies that $\Phi_{W^{\prime \prime}} \subseteq \Phi_{W^{\prime}}$ and $W^{\prime \prime} \subseteq W^{\prime}$. That is, $W^{\prime}$ is the meet of $\left\{W_{i}\right\}_{i \in I}$ as required.

Remarks. Given two elements of $\mathscr{W}$ each with a given expression as a conjugate of a standard facial subgroup, one can compute a similar expression for their meet and join in $\mathscr{W}$. In fact, as in the proof of 2.17 (c), one can reduce easily to the case 
in which the two subgroups are $W_{J}$ and $d W_{K} d^{-1}$ where $J, K$ are special facial subsets of $J$ and $d$ is of minimal length in $W_{J \cup J^{\perp}} d W_{K \cup K^{\perp}}$. Then by Kilmoyer's formula (Proposition 2.12) their meet is $W_{L}$ where $L:=\left(J \cap d K d^{-1}\right)_{\infty}$ is the union of all infinite type components of $J \cap d K d^{-1}$. By Lemma 2.17 and Lemma 8.5 . their join is $W_{M}$ where $W_{M}$ is the standard facial closure of $J \cup\{d\} \cup K$ i.e. $M$ is the smallest standard facial subset of $S$ containing $J \cup K \cup\left\{s_{1}, \ldots, s_{n}\right\}$ for some reduced expression $d=s_{1} \cdots s_{n}$ of $d$. We do not know if the standard facial and standard parabolic closures of $J \cup\{d\} \cup K$ coincide under these conditions.

10.3. Henceforward, extensive use will be made of the various notions of faces of cones as described in Appendix A, and especially the notions of semidual and dual pairs of pointed cones A.6 A.9.

By Theorem 5.1, $(\overline{\mathscr{Z}}, \mathscr{X})$ is a semidual pair of stable cones (with respect to the pairing $\langle-,-\rangle)$. It follows trivially that $P=(\mathscr{Z}, \mathscr{X})$ is also a semidual pair of (pointed) cones for the same pairing, satisfying the condition A.9 (i). Following A.6, we define the Galois connection corresponding to $P$ between the power sets $\mathscr{P}(\mathscr{Z})$ and $\mathscr{P}(\mathscr{X})$. It is specified by order-reversing maps

$$
A \mapsto A^{\dagger}:=\mathscr{X} \cap A^{\perp}: \mathscr{P}(\mathscr{Z}) \rightarrow \mathscr{P}(\mathscr{X})
$$

and

$$
B \mapsto B^{\#}:=\mathscr{Z} \cap B^{\perp}: \mathscr{P}(\mathscr{X}) \rightarrow \mathscr{P}(\mathscr{Z}) .
$$

The complete lattices $\operatorname{Ext}_{\neq \emptyset}(\mathscr{Z})$ and $\operatorname{Ext}_{\neq \emptyset}(\mathscr{X})$ of non-empty faces of $\mathscr{Z}$ and $\mathscr{X}$ are defined as in A.3. They contain (as subposets) the complete lattices of stable subsets (which we call stable faces) of $\mathscr{Z}$ and $\mathscr{X}$ for the above Galois connection.

For any reflection subgroup $W^{\prime}$ of $W$, define $\mathscr{X}\left(W^{\prime}\right):=\mathscr{X} \cap \Pi_{W^{\prime}}^{\perp}=\{x \in \mathscr{X} \mid$ $\left.x^{\perp} \cap \Phi \supseteq \Phi_{W^{\prime}}\right\}$. Observe that for any $x \in \mathscr{X}, x^{\perp} \cap \Phi=\Phi_{W^{\prime \prime}}$ for some facial reflection subgroup $W^{\prime \prime}$. Hence $x \in \mathscr{X}_{W^{\prime}}$ if and only if $W^{\prime \prime} \supseteq W^{\prime}$. The sets $\mathscr{X}\left(W^{\prime}\right)$ are primarily of interest here for $W^{\prime} \in \mathscr{W}$.

The following theorem summarizes the main results of this section.

Theorem. (a) $P=(\mathscr{Z}, \mathscr{X})$ is a dual pair of cones in the sense of Definition A.9.

(b) The map $W^{\prime} \mapsto \mathscr{Z}_{W^{\prime}}$ induces an isomorphism of complete lattices $\mathscr{W} \rightarrow$ $\operatorname{Ext}_{\neq \emptyset}(\mathscr{Z})$.

(c) The map $W^{\prime} \mapsto \mathscr{X}\left(W^{\prime}\right)$ induces an isomorphism of complete lattices $\mathscr{W} \rightarrow$ $\operatorname{Ext}_{\neq \emptyset}(\mathscr{X})^{\text {op }}$ (where ${ }^{\text {op }}$ indicates the opposite poset).

(d) For $W^{\prime} \in \mathscr{W}, \mathscr{Z}_{W^{\prime}}^{\dagger}=\mathscr{X}\left(W^{\prime}\right)$ and $\mathscr{X}\left(W^{\prime}\right)^{\#}=\mathscr{Z}_{W^{\prime}}$.

The proof of the theorem will occupy 10.410 .11 .

Remarks. (1) For $W^{\prime} \in \mathscr{W}, \mathscr{Z}_{W^{\prime}}$ is both the imaginary cone of $W^{\prime}$ and a face of $\mathscr{Z}$. However, the Tits cone of $W^{\prime}$ is $\mathscr{X}_{W^{\prime}} \supseteq \mathscr{X}$, whereas $\mathscr{X}\left(W^{\prime}\right) \subseteq \mathscr{X}$ is a face of $\mathscr{X}$.

(2) We discuss here the main known results in the Kac-Moody setting (these are analogous to, but not consequence of, the results established about the Tits cone here in the special case in which the simple roots are linearly independent). In the Kac-Moody setting, [35] studied exposed faces of the Tits cone attached to special standard parabolic subgroups, obtaining in particular formulae for their setwise and pointwise stabilizers. In [45, Kapitel 6] (see also [46]) it was shown that the exposed faces of the Tits cone are the $W$-translates of the special exposed faces 
defined in [35. Amongst other results, 38, 4.1] (see also [40]) gave another proof of this showing as well that any non-empty face (extreme subset) of the Tits cone is exposed. An algebraic description of meet and join of two faces of the Tits cone was given in [41, Theorem 4(b)] (the analogue here is given as Remark 10.2).

10.4. As in 5.7, $F_{\text {inf }}$ denotes the set of special facial subsets of $S$ (i.e. with no finite type components). For $I \subseteq S$, let $I^{\perp}:=\{r \in S \mid r \notin I, r s=s r$ for all $s \in I\}$ and let $\tilde{I} \subseteq I$ be such that $\Pi_{\tilde{I}}$ is the union of the infinite type components of $\Pi_{I}$.

Lemma. (a) For any facial subset $I$ of $S, \tilde{I}$ is a facial subset of $S$, and $\mathscr{Z}_{\tilde{I}}=$ $\mathscr{Z}_{I}$.

(b) The sets $w \mathscr{Z}_{I}$ where $I \in F_{\text {inf }}$ and $w \in W$ are faces of $\mathscr{Z}$.

(c) If $I \in F_{\mathrm{inf}}$, there exists $p \in \mathscr{K}_{I}$ such that the facial support of $p$ is $\Pi_{I}$ and such that for any $\alpha \in \Pi$, one has $\langle p, \alpha\rangle=0$ if and only if either $\alpha$ is in an affine component of $\Pi_{I}$ or $\alpha \in \Pi_{I^{\perp}}$.

(d) Let $I, J \in F_{\inf }$ with $I \subseteq J$. Then the normalizer of $W_{I}$ is $N\left(W_{I}\right)=$ $W_{I \cup I^{\perp}}=W_{I} W_{I^{\perp}}=W_{I^{\perp}} W_{I}$ and $N\left(W_{I}\right) N\left(W_{J}\right)=W_{I^{\perp}} W_{J}$.

(e) If $x, y \in W$ and $I, J \in F_{\text {inf }}$, the conditions (i)-(iii) below are equivalent:

(i) $x \mathscr{Z}_{I} \subseteq y \mathscr{Z}_{J}$.

(ii) $I \subseteq J$ and $x^{-1} y \in N\left(W_{I}\right) N\left(W_{J}\right)$.

(iii) $x W_{I} x^{-1} \subseteq y W_{J} y^{-1}$.

Proof. Part (a) follows from 8.5 (c) and $3.2(\mathrm{~d})$. Now $\mathscr{Z}=\mathscr{Z}_{S}$ itself is a stable face of $\mathscr{Z}$. For any proper facial subset $I \subseteq S$, we have (with notation as in 5.8) $\mathscr{Z} \subseteq \mathbb{R}_{\geq 0} \Pi \subseteq H_{\bar{\phi}_{I}}^{\geq 0}$ and $\mathscr{Z} \cap H_{\bar{\phi}_{I}}^{\overline{\bar{N}}}=\mathscr{Z} \cap \mathbb{R}_{I}=\mathscr{Z}_{I}$ is a face (in fact, an absolutely exposed face, in the terminology of Appendix A of $\mathscr{Z}$ by 3.4(b). Since $W$ acts on $\mathscr{Z}, w \mathscr{Z}_{I}$ is a stable face of $\mathscr{Z}$ for any $w \in W$ and facial $I \subseteq S$. By (a), $w \mathscr{Z}_{I}=w \mathscr{Z}_{\tilde{I}}$ proving (b).

Choose $p \in \mathscr{K}_{I}$ to satisfy (c) as follows. Let $I_{1}, \ldots, I_{n}$ be subsets of $I$ such that $\Pi_{I_{j}}$ are the irreducible components of $\Pi_{I}$. For each $i=1, \ldots, n$, choose by 4.5 some $0 \neq p_{i} \in \mathscr{K}_{i}$ such that $\Pi_{I_{i}}$ is a support of $p_{i}$ and, if $\Pi_{I_{i}}$ is indefinite, $\left\langle p_{i}, \alpha\right\rangle<0$ for all $\alpha \in \Pi_{I_{i}}$. If $\Pi_{I_{i}}$ is affine, then $p_{i}$ necessarily is a representative of its isotropic ray. Set $p=p_{1}+\ldots+p_{n}$. Clearly, $\Pi_{I}$ is a support of $p$; hence $\Pi_{I}$ is the facial support of $p$ since $I$ is facial. We have $\langle p, \alpha\rangle \leq 0$ for all $\alpha \in \Pi \backslash \Pi_{I}$, with equality if and only if $s_{\alpha} \in I^{\perp}$. Summarizing, for any $\alpha \in \Pi$, we have

$$
\left\{\begin{array}{l}
\langle p, \alpha\rangle=0, \quad \text { if } \alpha \in \Pi_{I^{\perp}}, \text { or } \alpha \in \Pi_{I_{i}} \text { with } \Pi_{I_{i}} \text { affine } \\
\langle p, \alpha\rangle<0, \quad \text { otherwise. }
\end{array}\right.
$$

This proves (c).

It is well known (see [7]) that for any reflection subgroup $W^{\prime}$, one has $N\left(W^{\prime}\right)=$ $W^{\prime} N_{W}\left(\Pi_{W^{\prime}}\right)$ where $N_{W}\left(\Pi_{W^{\prime}}\right)=\left\{w \in W \mid w \Pi_{W^{\prime}}=\Pi_{W^{\prime}}\right\}$ (this also follows from 1.6). Since all irreducible components of $\Pi_{I}$ are of infinite type, it is an easy consequence of 1.15 (see [16]) that if $x \in W$ and $x\left(\Pi_{I}\right)=\Pi_{J}$ where $J \subseteq S$, then $I=J$ and $x \in W_{I^{\perp}}$. Hence $N_{W}\left(\Pi_{I}\right)=W_{I^{\perp}}, N\left(W_{I}\right)=W_{I} N\left(\Pi_{I}\right)=W_{I} W_{I^{\perp}}=$ $W_{I \cup I^{\perp}}$ and

$$
N\left(W_{I}\right) N\left(W_{J}\right)=W_{I^{\perp}} W_{I} W_{J^{\perp}} W_{J}=W_{I^{\perp}} W_{J \perp} W_{I} W_{J}=W_{I^{\perp}} W_{J}
$$

if $I \subseteq J$. This proves (d).

Next, we prove (e). To show (i) implies (ii), it will suffice to show that if $\mathscr{Z}_{I} \subseteq$ $y \mathscr{Z}_{J}$, then $I \subseteq J$ and $y \in N\left(W_{I}\right) N\left(W_{J}\right)$. Choose $p$ as in (c). Then $p \in \mathscr{Z}_{I} \subseteq y \mathscr{Z}_{J}=$ 
$y W_{J} \mathscr{K}_{J}$, so $p=y w k$ for some $w \in W_{J}$ and $k \in \mathscr{K}_{J}$. Since $\mathscr{K}_{I}, \mathscr{K}_{J} \subseteq \mathscr{K} \subseteq-\mathscr{C}$ by Proposition 3.4(a), we get $p, k \in-\mathscr{C}$. This implies by Lemma 1.10 that $p=k$ and $y w \in W_{L}$ where $\Pi_{L}=\{\alpha \in \Pi \mid\langle p, \alpha\rangle=0\}$. Clearly $W_{L} \subseteq N\left(W_{I}\right)$ so $y w \in N\left(W_{I}\right)$. Therefore $y=(y w) w^{-1} \in N\left(W_{I}\right) W_{J} \subseteq N\left(W_{I}\right) N\left(W_{J}\right)$. Also, since $p=k \in \mathscr{K}_{J}, p$ has a support $I$, and $J$ is facial, it follows that $I \subseteq J$. This completes the proof that (i) implies (ii). It is easy to see that (ii) implies (iii). If (iii) holds, then $x \mathscr{Z}_{I}=\mathscr{Z}_{x W_{I} x^{-1}} \subseteq \mathscr{Z}_{y W_{J} y^{-1}}=y \mathscr{Z}_{J}$, by Proposition 3.4 (a) and Theorem 6.3. so (iii) implies (i). This proves (e).

10.5. Partially order the power sets $\mathscr{P}(\mathscr{Z})$ and $\mathscr{P}(\mathscr{X})$ by inclusion.

Lemma. (a) The map $W^{\prime} \mapsto \mathscr{Z}_{W^{\prime}}: \mathscr{W} \cong\left\{\mathscr{Z}_{W^{\prime}} \mid W^{\prime} \in \mathscr{W}\right\}$ is a poset isomorphism.

(b) The map $W^{\prime} \mapsto \mathscr{X}\left(W^{\prime}\right): \mathscr{W}^{\mathrm{op}} \stackrel{\cong}{\rightrightarrows}\left\{\mathscr{X}\left(W^{\prime}\right) \mid W^{\prime} \in \mathscr{W}\right\}$ is a poset isomorphism.

Proof. Observe that $\mathscr{W}=\left\{w W_{I} w^{-1} \mid w \in W, I \in F_{\text {all }}\right\}$. Therefore, (a) follows directly from the equivalence of (i) and (iii) in Lemma 10.4(e). For (b), note that for any facial subgroup $W^{\prime}$ of $W$, there exists $z \in \mathscr{X}$ such that $\Phi \cap z^{\perp}=\Phi_{W^{\prime}}$. It follows from this that for facial subgroups $W^{\prime}, W^{\prime \prime}$ of $W$, one has $\mathscr{X}\left(W^{\prime}\right) \subseteq \mathscr{X}\left(W^{\prime \prime}\right)$ if and only if $W^{\prime \prime} \subseteq W^{\prime}$. Restricting to $W^{\prime}, W^{\prime \prime} \in \mathscr{W}$ gives (b).

10.6. In 10.6 10.7 we fix $W^{\prime} \in \mathscr{W}$ and let facial support of elements of $\mathbb{R}_{\geq 0} \Pi_{W^{\prime}}$ be taken with respect to $\Pi_{W^{\prime}}$ unless otherwise indicated.

Lemma. For any $z \in \mathscr{Z}_{W^{\prime}}$, there are two mutually exclusive possibilities: either $z$ lies in $\mathscr{Z}_{W^{\prime \prime}}$ for some $W^{\prime \prime} \in \mathscr{W}$ with $W^{\prime \prime} \subsetneq W^{\prime}$, or wz has facial support $\Pi_{W^{\prime}}$ for all $w \in W^{\prime}$.

Proof. Using the $W^{\prime}$ action, assume without loss of generality that $z=k \in \mathscr{K}_{W^{\prime}}$. Observe that the facial support $\Delta$ of $k$ (with respect to $\Pi_{W^{\prime}}$ ) is of the form $\Delta=\Pi_{W^{\prime \prime}}$ for some $W^{\prime \prime} \in \mathscr{W}$ with $W^{\prime \prime}$ standard parabolic (even standard facial) in $W^{\prime}$. In fact, let $\Delta^{\prime}$ (resp., $\Delta^{\prime \prime}$ ) be the union of the infinite (resp., finite) type components of $\Delta$. Since $\Delta$ is standard facial in $\Pi_{W^{\prime}}$, and $k \in \mathscr{K}$, it follows using Lemma 3.4 . Proposition $3.2(\mathrm{~d})$ and 4.5 that

$$
k \in \mathbb{R}_{\geq 0} \Delta \cap \mathscr{K}_{W^{\prime}}=\mathscr{K}_{W_{\Delta}}=\mathscr{K}_{W_{\Delta^{\prime}}}+\mathscr{K}_{W_{\Delta^{\prime \prime}}}=\mathscr{K}_{W_{\Delta^{\prime}}} .
$$

One has (e.g. by 2.13 and 2.9 that $\mathbb{R} \Delta=\mathbb{R} \Delta^{\prime} \oplus \mathbb{R} \Delta^{\prime \prime}$ (orthogonal direct sum) where $\Delta^{\prime \prime}$ is linearly independent. This implies that any support of $k$ is contained in $\Delta^{\prime}$, so $\Delta^{\prime}=\Delta$ has no finite type components. Then $W^{\prime \prime}=W_{\Delta} \in \mathscr{W}$ by Lemma 10.1 (b). Now if $W^{\prime \prime} \neq W^{\prime}$, then $k$ lies in a proper face $\mathscr{Z}_{W^{\prime \prime}}$ of $\mathscr{Z}_{W^{\prime}}$ and has proper facial support $\Delta \subsetneq \Pi_{W^{\prime}}$ in $\Pi_{W^{\prime}}$ On the other hand, if $W^{\prime \prime}=W^{\prime}$, then $w k$ has facial support $\Pi_{W^{\prime}}$ for all $w \in W^{\prime}$, since $w k-k \in \mathbb{R}_{\geq 0} \Pi_{W^{\prime}}$ by 1.10 . It is clear from the proof that the two possibilities mentioned in the statement of the Lemma are mutually exclusive, and the proof of the Lemma is complete.

10.7. The next lemma, in which for conciseness, $C^{\circ}:=\operatorname{ri}(C)$ for any cone $C$, characterizes the relative interior of $\mathscr{Z}_{W^{\prime}}$.

Lemma. Let $W^{\prime} \in \mathscr{W}$ and $z \in \mathscr{Z}_{W^{\prime}}$. Write $z=w k$ with $w \in W^{\prime}$ and $k \in \mathscr{K}_{W^{\prime}}$ Then the following conditions are equivalent:

$$
\text { (i) } z \in \mathscr{Z}_{W^{\prime}}^{\circ} \text {. }
$$


(ii) $w z$ has facial support $\Pi_{W^{\prime}}$ for all $w \in W^{\prime}$.

(iii) $w z$ has facial support $\Pi_{w W^{\prime} w^{-1}}$ with respect to $\Pi_{w W^{\prime} w^{-1}}$, for all $w \in W$.

(iv) the facial support of $k$ with resect to $\Pi_{W^{\prime}}$ is $\Pi_{W^{\prime}}$.

Proof. Making use of the $W^{\prime}$-action, we may assume that $z=k \in \mathscr{K}_{W^{\prime}}$. Since no point of a proper face of $\mathscr{Z}_{W^{\prime}}$ is in $\mathscr{Z}_{W^{\prime}}^{\circ}$, the preceding Lemma shows that (i) implies (ii). Clearly (ii) implies (iv). Conditions (ii) and (iii) are easily seen to be equivalent using 1.6 1.7 and Proposition 3.2(e). To complete the proof, we show that (iv) implies (i). Using the $W^{\prime}$-action, it will suffice to show that $k \in \mathscr{Z}_{W^{\prime}}^{\circ}$. Now by definition of $\mathscr{W}, \Pi_{W^{\prime}}$ has only infinite type components. Let $\Omega$ denote the union of all indefinite type components of $\Pi_{W^{\prime}}$ and $\Gamma$ denote the union of all other (affine type) components of $\Pi_{W^{\prime}}$. Write $k=\sum_{\alpha \in \Pi_{W^{\prime}}} c_{\alpha} \alpha$ where all $c_{\alpha}>0$. By the proof of $3.2(\mathrm{~d}), k_{\Omega}:=\sum_{\alpha \in \Omega} c_{\alpha} \alpha \in \mathscr{K}_{W_{\Omega}}$ and $k_{\Gamma}:=\sum_{\alpha \in \Gamma} c_{\alpha} \alpha \in \mathscr{K}_{W_{\Gamma}}$. Further, from Lemma 8.4 it follows that for each component $\Gamma^{\prime}$ of $\Gamma, k_{\Gamma^{\prime}}:=\sum_{\alpha \in \Gamma^{\prime}} c_{\alpha} \alpha$ is a representative of the isotropic ray $\mathscr{K}_{W_{\Gamma^{\prime}}}$ of $W_{\Gamma^{\prime}}$. From 8.6. $\mathscr{Z}_{\Gamma}=\mathscr{K}_{\Gamma}=\sum_{\Gamma^{\prime}} \mathscr{K}_{W_{\Gamma^{\prime}}}$ and so clearly $k_{\Gamma} \in \mathscr{Z}_{\Gamma}^{\circ}$. It will be enough to show that $k_{\Omega} \in \mathscr{Z}_{\Omega}^{\circ}$. For then $k=k_{\Omega}+k_{\Gamma} \in \mathscr{Z}_{\Omega}^{\circ}+\mathscr{Z}_{\Gamma}^{\circ} \subseteq \mathscr{Z}_{W^{\prime}}^{\circ}($ using $3.2(\mathrm{~d})$ and A.3.14) $)$.

To prove that $k_{\Omega} \in \mathscr{Z}_{\Omega}^{\circ}$, we may and do assume without loss of generality that $\Pi=\Pi_{W^{\prime}}=\Omega$, so $k_{\Omega}=k$ and $\Pi_{W^{\prime}}$ has only indefinite components. By 4.5. $\mathbb{R} \mathscr{Z}_{W^{\prime}}=\mathbb{R}_{W^{\prime}}$ and by Lemma $8.4(\mathrm{~d}), \Delta:=k^{\perp} \cap \Pi_{W^{\prime}}$ has only finite type components. Let $W^{\prime \prime}:=W_{\Delta}$. We choose an open neighborhood $U$ of $k$ in $V$ which does not intersect the hyperplane $\alpha^{\perp}$ for any $\alpha \in \Pi_{W^{\prime}} \backslash \Delta$, and such that $U^{\prime}:=U \cap \mathbb{R}_{W^{\prime}} \subseteq \mathbb{R}_{\geq 0} \Pi_{W^{\prime}}$ (which is possible since $k \in\left(\mathbb{R}_{\geq 0} \Pi_{W^{\prime}}\right)^{\circ}$ ). Since $k$ is $W^{\prime \prime}$-stable, we may assume without loss of generality that $U$ (and hence $U^{\prime}$ ) is $W^{\prime \prime}$-stable also. We have $\left\langle\Pi_{W^{\prime}} \backslash \Delta, U\right\rangle \subseteq \mathbb{R}_{<0}$ since $\left\langle\Pi_{W^{\prime}} \backslash \Delta, k\right\rangle \subseteq \mathbb{R}_{<0}$. We claim that $U^{\prime}$ is an open neighborhood of $k$ in $\mathbb{R} \mathscr{Z}_{W^{\prime}}$ with $U^{\prime} \subseteq \mathscr{Z}_{W^{\prime}}$. By construction, $U^{\prime}$ is an open neighborhood of $k$ in $\mathbb{R} \mathscr{Z}_{W^{\prime}}$. To see that $U^{\prime} \subseteq \mathscr{Z}_{W^{\prime}}$, recall that since $W^{\prime \prime}$ is finite, we have $\mathscr{X}_{W^{\prime \prime}}=\cup_{w \in W^{\prime \prime}} w \mathscr{C}=V$ by $1.10(\mathrm{~h})$. So if $u \in U^{\prime}$, there is $u^{\prime} \in-\mathscr{C}_{W^{\prime \prime}}$ and $w \in W^{\prime \prime}$ with $u=w u^{\prime}$. By $W^{\prime \prime}$-invariance of $U^{\prime}$, we have $u^{\prime} \in U^{\prime}$ also, so $\left\langle\Pi_{W^{\prime}} \backslash \Delta, u^{\prime}\right\rangle \subseteq \mathbb{R}_{<0}$. We have $\left\langle u^{\prime}, \Delta\right\rangle \subseteq \mathbb{R}_{\leq 0}$ since $u^{\prime} \in-\mathscr{C}_{W^{\prime \prime}}$. It follows that $u^{\prime} \in \mathbb{R}_{\geq 0} \Pi_{W^{\prime}} \cap-\mathscr{C}_{W^{\prime}}=\mathscr{K}_{W^{\prime}}$ and $u=w u^{\prime} \in \bar{W}^{\prime} \mathscr{K}_{W^{\prime}}=\mathscr{Z}_{W^{\prime}}$ since $w \in W^{\prime \prime} \subseteq W^{\prime}$. This shows that $U^{\prime} \subseteq \mathbb{R} Z_{W^{\prime}}$ as claimed, and completes the proof of the Lemma.

10.8. The statement below uses the notation $\mathscr{C}(I)$ defined in 2.3 .

Lemma. Let $W^{\prime} \in \mathscr{W}$, and write $W^{\prime}=w W_{I} w^{-1}$ for some $w \in W$ and $I \in F_{\mathrm{inf}}$.

(a) Let $z \in \mathscr{Z}_{W^{\prime}}^{\circ}$. Then $\{z\}^{\dagger}=\left(\mathscr{Z}_{W^{\prime}}\right)^{\dagger}=\mathscr{X} \cap\left(\mathbb{R} \Pi_{W^{\prime}}\right)^{\perp}=\mathscr{X}\left(W^{\prime}\right)$.

(b) Choose $v \in \mathscr{C}(I)$. Then $\mathscr{Z}_{W^{\prime}}=\left(\mathscr{X}\left(W^{\prime}\right)\right)^{\#}=(w \mathscr{C}(I))^{\#}=\{w v\}^{\#}$.

(c) $\mathscr{Z}_{W^{\prime}}$ is a stable face of $\mathscr{Z}$ dual (with respect to the Galois connection from $P)$ to the stable face $\mathscr{X}\left(W^{\prime}\right)$ of $\mathscr{X}$.

Proof. In (a), it is obvious from the definitions that

$$
\{z\}^{\dagger} \supseteq\left(\mathscr{Z}_{W^{\prime}}\right)^{\dagger} \supseteq \mathscr{X} \cap\left(\mathbb{R} \Pi_{W^{\prime}}\right)^{\perp}=\mathscr{X}\left(W^{\prime}\right) .
$$

Using the $W$-action, we may assume for the proof of the reverse inclusions that $W^{\prime}$ is standard facial, say $W^{\prime}=W_{I}$ where $I \in F_{\text {inf }}$. Let $x \in\{z\}^{\dagger}=\mathscr{X} \cap z^{\perp}$. Choose $w \in W$ so $w x \in \mathscr{C}$. Thus, $w x \in(w z)^{\perp}$. By Lemma 10.7, $w z=\sum_{\alpha \in \Pi_{w W^{\prime} w^{-1}}} c_{\alpha} \alpha$ with all $c_{\alpha}>0$. It follows that $w x \in\left(\Pi_{w W^{\prime} w^{-1}}\right)^{\perp}$ and so $x \in\left(\Pi_{W^{\prime}}\right)^{\perp} \cap \mathscr{X}$. This 
proves (a). In (b), we have $v \in \mathscr{C}_{I} \subseteq \mathscr{C} \cap \Pi_{I}^{\perp} \subseteq \mathscr{X} \cap \Pi_{I}^{\perp}=\mathscr{X}\left(W_{I}\right)$ and thus

$$
\begin{aligned}
\mathscr{Z}_{W_{I}} \subseteq\left(\mathscr{X}\left(W_{I}\right)\right)^{\#} \subseteq(\mathscr{C}(I))^{\#} \subseteq\{v\}^{\#}=\mathscr{Z} \cap v^{\perp}= \\
\mathscr{Z} \cap \mathbb{R}_{\geq 0} \Pi \cap v^{\perp}=\mathscr{Z} \cap \mathbb{R}_{\geq 0} \Pi_{I}=\mathscr{Z}_{W_{I}}
\end{aligned}
$$

where the first inclusion comes from (a) and the last equality from Lemma 3.4 Hence equality holds throughout. Acting on the resulting equation by $w$ gives (b). Part (c) is immediate from (a)-(b) and the definitions.

10.9. For $W^{\prime} \in \mathscr{W}$, let $\mathscr{X}^{\prime}\left(W^{\prime}\right) \subseteq \mathscr{X}\left(W^{\prime}\right)$ denote the set of all $x \in \mathscr{X}$ such that $x^{\perp} \cap \Phi=\Phi_{W^{\prime \prime}}$ where $W^{\prime \prime}$ is the facial reflection subgroup such that $\Pi_{W^{\prime}}$ is the union of all infinite type components of $\Pi_{W^{\prime \prime}}$. It is easy to see that for $W^{\prime} \in \mathscr{W}$, we have

$$
\mathscr{X}\left(W^{\prime}\right)=\bigcup_{\substack{W^{\prime \prime} \in \mathscr{W} \\ W^{\prime \prime} \supseteq W^{\prime}}} \mathscr{X}^{\prime}\left(W^{\prime \prime}\right) .
$$

Lemma. If $W^{\prime} \in \mathscr{W}$, then $\mathscr{X}\left(W^{\prime}\right)^{\circ}=\mathscr{X}^{\prime}\left(W^{\prime}\right)$.

Proof. Since $\mathscr{X}^{\prime}\left(W^{\prime \prime}\right)$ is in the proper face $\mathscr{X}\left(W^{\prime \prime}\right)$ (and therefore in the relative boundary) of $\mathscr{X}\left(W^{\prime}\right)$ for $W^{\prime \prime} \in \mathscr{W}$ with $W^{\prime \prime} \supsetneq W^{\prime}$, we have $\mathscr{X}\left(W^{\prime}\right)^{\circ} \subseteq \mathscr{X}^{\prime}\left(W^{\prime}\right)$. Hence it remains to prove that if $v \in \mathscr{X}^{\prime}\left(W^{\prime}\right)$, there is an open neighborhood $U$ of $v$ in $\mathscr{X}\left(W^{\prime}\right)$ such that $U \subseteq \mathscr{X}^{\prime}\left(W^{\prime}\right)$. Choose $w \in W$ with $x:=w v \in \mathscr{C}$. Let $W_{I}$ be the stabilizer of $x$ in $W$ and $\tilde{I} \subseteq I$ be as in 10.4. Then $w W^{\prime} w^{-1}=W_{\tilde{I}}$, $x=w v \in w \mathscr{X}^{\prime}\left(W^{\prime}\right)=\mathscr{X}^{\prime}\left(W_{\tilde{I}}\right)$ and $w \mathscr{X}\left(W^{\prime}\right)=\mathscr{X}\left(\bar{W}_{\tilde{I}}\right)$.

Set $J=I \backslash \tilde{I}$, so $W_{J}$ is finite and $J$ is the union of the finite type connected components of $I$. We have $\Pi_{I}=x^{\perp} \cap \Pi$. Hence we may choose an open neighborhood $U^{\prime}$ of $x$ in $V$ such that $\left\langle U^{\prime}, \Pi \backslash \Pi_{I}\right\rangle \subseteq \mathbb{R}_{>0}$. Since $W_{J}$ stabilizes $x$, we may assume without loss of generality, that $U^{\prime}$ is $W_{J}$-invariant.

We claim that $U^{\prime \prime}:=U^{\prime} \cap \mathscr{X}\left(W_{\tilde{I}}\right)$ is an open neighborhood of $x$ contained in $\mathscr{X}^{\prime}\left(W_{\tilde{I}}\right)$. Clearly, $U^{\prime \prime}$ is open in $\mathscr{X}\left(W_{\tilde{I}}\right)$. Let $p \in U^{\prime \prime}$. Since $W_{J}$ is finite, we have $\mathscr{X}_{W_{J}}=V \ni p$, so there is some $y \in W_{J}$ such that $y p \in \mathscr{C}_{W_{J}}$. This means that $\left\langle y p, \Pi_{J}\right\rangle \subseteq \mathbb{R}_{\geq 0}$. Also, $\left\langle y p, \Pi \backslash \Pi_{I}\right\rangle \subseteq \mathbb{R}_{>0}$ since $y p \in U^{\prime}$ by $W_{J}$-invariance of $U^{\prime}$. We have $p^{\perp} \cap \Phi=\Phi_{W^{\prime \prime \prime}}$ for some facial reflection subgroup $W^{\prime \prime \prime}$. Since $p \in \mathscr{X}\left(W_{\tilde{I}}\right)$, we have $W^{\prime \prime \prime} \supseteq W_{\tilde{I}}$. Hence $(y p)^{\perp} \cap \Phi=y \Phi_{W^{\prime \prime \prime}} \supseteq y \Phi_{\tilde{I}}=\Phi_{\tilde{I}}$ since $y \in W_{J}$. This shows that $\left\langle y p, \Pi_{\tilde{I}}\right\rangle=0$. Since $\Pi=\Pi_{J} \cup\left(\Pi \backslash \Pi_{I}\right) \cup \Pi_{\tilde{I}}$, we have now seen that $y p \in \mathscr{C} \subseteq \mathscr{X}$ with $\Pi_{\tilde{I}} \subseteq(y p)^{\perp} \cap \Pi \subseteq \Pi_{I}$. Hence $y p \in \mathscr{X}^{\prime}\left(W_{\tilde{I}}\right)$ and $p \in \mathscr{X}^{\prime}\left(y^{-1} W_{\tilde{I}} y\right)=\mathscr{X}^{\prime}\left(W_{\tilde{I}}\right)$. We conclude that $U^{\prime \prime} \subseteq \mathscr{X}^{\prime}\left(W_{\tilde{I}}\right)$ as claimed. Finally, $U:=w^{-1} U^{\prime \prime}$ is an open neighborhood of $w^{-1} x=v$ in $w^{-1}\left(\mathscr{X}\left(W_{\tilde{I}}\right)\right)=\mathscr{X}\left(W^{\prime}\right)$ such that $U \subseteq w^{-1}\left(\mathscr{X}^{\prime}\left(W_{\tilde{I}}\right)\right)=\mathscr{X}^{\prime}\left(W^{\prime}\right)$. This completes the proof.

10.10. The following is the last lemma required for the proof of Theorem 10.3 .

Lemma. (a) The sets $\mathscr{X}\left(W^{\prime}\right)^{\circ}$ for $W^{\prime} \in \mathscr{W}$ are pairwise disjoint and have union $\mathscr{X}$. Further, for $W^{\prime} \in \mathscr{W}$, we have

$$
\mathscr{X}\left(W^{\prime}\right)=\bigcup_{\substack{W^{\prime \prime} \subseteq \mathscr{W} \\ W^{\prime}}} \mathscr{X}\left(W^{\prime \prime}\right)^{\circ} .
$$

(b) The sets $\mathscr{Z}_{W^{\prime}}^{\circ}$ for $W^{\prime} \in \mathscr{W}$ are pairwise disjoint, and have union $\mathscr{Z}$. Further, for $W^{\prime} \in \mathscr{W}$,

$$
\mathscr{Z}_{W^{\prime}}=\bigcup_{W^{\prime \prime} \subseteq W^{\prime}} \mathscr{Z}_{W^{\prime \prime}}^{\circ}
$$


Proof. Part (a) follows directly from 10.9 and the definitions.

For (b), suppose first that $z \in \mathscr{Z}_{W^{\prime}}^{\circ} \cap \mathscr{Z}_{W^{\prime \prime}}^{\circ}$ where $W^{\prime}, W^{\prime \prime} \in \mathscr{Z}_{W^{\prime}}$. Without loss of generality, $W^{\prime} \nsubseteq W^{\prime \prime}$. By Corollary 6.4, $z \in \mathscr{Z}_{W^{\prime}} \cap \mathscr{Z}_{W^{\prime \prime}}=\mathscr{Z}_{W^{\prime \prime \prime}}$ where $W^{\prime \prime \prime} \in \mathscr{W}$ has $\Pi_{W^{\prime \prime \prime}}$ equal to the union of the infinite type components of $\Pi_{W^{\prime} \cap W^{\prime \prime}}$. But then, since $W^{\prime \prime \prime} \subsetneq W^{\prime}$, it follows by Lemma 10.7 that $z \notin \mathscr{Z}_{W^{\prime}}^{\circ}$, a contradiction. Hence the sets $\mathscr{Z}_{W^{\prime}}^{\circ}$ for $w^{\prime} \in \mathscr{W}$ are pairwise disjoint.

Now let $z \in \mathscr{Z}_{W^{\prime}}$ where $W^{\prime} \in \mathscr{W}$. Choose a minimal element $W^{\prime \prime} \in \mathscr{W}$ with $W^{\prime \prime} \subseteq W^{\prime}$ and $z \in \mathscr{Z}_{W^{\prime \prime}}$. By Lemma 10.7 again, we have $z \in \mathscr{Z}_{W^{\prime \prime}}^{\circ}$. Hence

$$
\mathscr{Z}_{W^{\prime}} \subseteq \bigcup_{W^{\prime \prime} \subseteq W^{\prime}} \mathscr{Z}_{W^{\prime \prime}}^{\circ}
$$

The reverse inclusion holds since for $W^{\prime \prime} \in \mathscr{W}$ with $W^{\prime \prime} \subseteq W^{\prime}, \mathscr{Z}_{W^{\prime \prime}}^{\circ} \subseteq \mathscr{Z}_{W^{\prime \prime}} \subseteq \mathscr{Z}_{W^{\prime}}$ by Theorem 6.3 .

10.11. Proof of Theorem 10.3. Lemma 10.8 (c) states that for $W^{\prime} \in \mathscr{W}, \mathscr{Z}_{W^{\prime}}$ is a stable face of $\mathscr{Z}$ dual to the stable face $\mathscr{X}\left(W^{\prime}\right)$ of $\mathscr{X}$. Now by A.3.6, $\mathscr{Z}$ is the disjoint union of the relative interiors of its non-empty faces; comparing with Lemma 10.10 (b) shows that $\operatorname{Ext}_{\neq \emptyset}(\mathscr{Z})=\left\{\mathscr{Z}_{W^{\prime}} \mid W^{\prime} \in \mathscr{W}\right\}$ and that every non-empty face of $\mathscr{Z}$ is a stable face. Similarly, Lemma 10.10(b) implies that $\operatorname{Ext}_{\neq \emptyset}(\mathscr{Z})=\left\{\mathscr{X}_{W^{\prime}} \mid W^{\prime} \in \mathscr{W}\right\}$ and that every non-empty face of $\mathscr{X}$ is a stable face. Together, these verify the condition A.9(ii), and validity of A.9(i) has already been noted. This proves 10.3(a). Now 10.3(b)-(c) follow from Lemma 10.5(a)-(b) and 10.3 (d) follows from Lemma 10.8. This completes the proof of Theorem 10.3 .

10.12. We conclude this section with the following observation.

Corollary. Let $W^{\prime}$ be a finitely generated reflection subgroup of $W$. Then the map defined by $F \mapsto F \cap \mathscr{Z}_{W^{\prime}}: \operatorname{Ext}_{\neq \emptyset}(\mathscr{Z}) \rightarrow \operatorname{Ext}_{\neq \emptyset}\left(\mathscr{Z}_{W^{\prime}}\right)$, for $F$ a face of $\mathscr{Z}_{W}$, preserves meets of arbitrary subsets of $\operatorname{Ext}_{\neq \emptyset}(\mathscr{Z})$.

Proof. This follows since for any non-empty face $F$ of $\mathscr{Z}, F \cap \mathscr{Z}_{W^{\prime}}$ is a non-empty face of $\mathscr{Z}_{W^{\prime}}$ by Corollary 6.4 and the results of this section, and the meet of faces in either lattice is given by their intersection.

\section{Facial Closures of Reflection Subgroups}

This subsection describes results which lead to certain algorithms for computing facial closures of reflection subgroups. Since specification of general real numbers in finite terms and and algorithmic computation with them is impossible, we shall assume that oracles are available for performing arithmetic computations with arbitrary real numbers and determining their signs. By an $\mathbb{R}$-algorithm, we shall mean an algorithm with any necessary real arithmetic and sign or equality tests handled by such oracles. We shall not be more precise, as we shall use this terminology only for informal comments.

Under mild additional conditions (loosely, that $(V,\langle-,-\rangle)$ and $(\Phi, \Pi)$ are defined, in an obvious natural sense, over a subfield $K$ of $\mathbb{R}$ with algorithmically computable arithmetic operations and sign and equality tests), these $\mathbb{R}$-algorithms readily adapt to give bona-fide (finite, terminating) algorithms. The main point is to replace statements involving the various subsets of $V$ (especially $V$ and cones $\left.\mathbb{R}_{\geq 0} \Pi, \mathscr{C}, \mathscr{X}, \mathscr{K}, \mathscr{Z}\right)$ appearing in the theory by statements involving the subsets 
of their $K$-points. We note that any finite extension field of $\mathbb{Q}$ in $\mathbb{R}$ is a "computable" field in this sense, and each finite rank Coxeter group is associated to some $(V,\langle-,-\rangle)$ and $(\Phi, \Pi)$ defined over a suitable computable field $K)$.

11.1. The first lemma gives rise to an $\mathbb{R}$-algorithm which determines, for $v \in \mathscr{C}$ (resp., $v \in \mathscr{Z}$ ) some $w \in W$ with $w v \in \mathscr{C}$ (resp., $w v \in \mathscr{K}$ ).

Lemma. For $v \in \mathscr{X}$, let $n_{v}:=\left|\left\{\alpha \in \Phi_{+} \mid\langle v, \alpha\rangle<0\right\}\right| \in \mathbb{N}$.

(a) $n_{v}=0$ if and only if $v \in \mathscr{C}$.

(b) If $n_{v}>0$, then there is $\alpha \in \Pi$ with $\langle\alpha, v\rangle<0$, and then $s_{\alpha} v \in \mathscr{X}$ with $n_{s_{\alpha} v}=n_{v}-1$. If $w^{\prime} \in W$ with $w^{\prime}\left(s_{\alpha} v\right) \in \mathscr{C}$, then $w v \in \mathscr{C}$ where $w=w^{\prime} s_{\alpha}$.

(c) Let $v \in \mathscr{Z}$. Then $-v \in \mathscr{X}$. Further, for $w \in W, w v \in \mathscr{K}$ if and only if $w(-v) \in \mathscr{C}$.

Proof. Parts (a)-(b) are assertions involved in a standard proof of 1.10(a) (see [33]); the main point for (b) is that $s_{\alpha}$ fixes the set $\Phi_{+} \backslash\{\alpha\}$ of positive roots. Part (c) follows readily from the definitions since $w v \in \mathbb{R}_{\geq 0} \Pi$ for all $w \in W$.

11.2. There are standard $\mathbb{R}$-algorithms of convex geometry which determine the facial subsets of $\Pi$ and the facial support of elements $v \in \mathbb{R}_{\geq 0} \Pi$ (for any $v$ specified as a non-negative $\mathbb{R}$-linear combination of elements of $\Pi$ ). Given a finitely generated reflection subgroup $W^{\prime}$, specified by a finite set of reflections generating it, there are algorithms to compute $\chi\left(W^{\prime}\right)$ in [17 and hence there are $\mathbb{R}$-algorithms for computing $\Pi_{W^{\prime}}$. We will also use that for $w \in W$, algorithms based on 1.6]1.7 enable one to compute the minimal length element of $W^{\prime} w$ for $w \in W$, and in particular, compute $\chi\left(w^{-1} W^{\prime} w\right)$ from $\chi\left(W^{\prime}\right)$.

Given $\Pi_{W^{\prime}}$, standard $\mathbb{R}$-algorithms of convex geometry enable one to compute the polyhedral cone $\mathscr{K}_{W^{\prime}}$ (specified either by the inequalities defining its minimal set of closed supporting half-spaces, or by a set of representatives of its extreme rays). In turn from this, one may give an $\mathbb{R}$-algorithm to determine a point of $\mathscr{K}_{W^{\prime}}^{\circ}$ (as a sum of representatives of the all the extreme rays of $\mathscr{K}_{W^{\prime}}$ is in $\mathscr{K}_{W^{\prime}}^{\circ}$ ). Since $\mathscr{K}_{W^{\prime}}^{\circ} \subseteq \mathscr{Z}_{W^{\prime}}^{\circ}$, it follows that there is a $\mathbb{R}$-algorithm to determine a point of $\mathscr{Z}_{W^{\prime}}^{\circ}$.

Generally, when we say that there is an $\mathbb{R}$-algorithm to compute a finitelygenerated reflection subgroup $W^{\prime \prime}$ from certain data, we mean that there is a $\mathbb{R}$ algorithm to compute $\chi\left(W^{\prime \prime}\right)$ from that data. We will also use in 11.5 the fact that there are $\mathbb{R}$-algorithms to determine, from $\chi\left(W^{\prime}\right)$ for any any finitely-generated reflection subgroup $W^{\prime}$, a subset $J \subseteq S$ and element $x \in W$ such that $x W_{J} x^{-1}$ is the parabolic closure of $W^{\prime}([20]$ ). This latter algorithm is sophisticated (compared to others discussed here), as it depends on the solvability of the conjugacy problem for Coxeter groups, which was for some time an open problem (see [34]). A simpler algorithm is known for computing parabolic closures of finite (reflection) subgroups (see [34]), and this will suffice for the results through 11.4

11.3. The next Lemma gives rise to an $\mathbb{R}$-algorithm to determine, for $v \in \mathscr{Z}$, the element $W^{\prime} \in \mathscr{W}$ with $v \in \mathscr{Z}_{W^{\prime}}^{\circ}$. It in fact determines an expression for $W^{\prime}$ as a $W$-conjugate of a standard facial subgroup $W_{I}$. Note from Lemma A.3 that $\mathscr{Z}_{W^{\prime}}$ is the minimum face of $\mathscr{Z}$ containing $v$.

Lemma. Let $v \in \mathscr{Z}$. Choose $w \in W$ with $w v \in \mathscr{K}$, and let the facial support of wv be $\Pi_{I}$ where $I \subseteq S$ is facial. Then $W^{\prime}:=w^{-1} W_{I} w \in \mathscr{W}$ and $v \in \mathscr{Z}_{W^{\prime}}^{\circ}$. 
Proof. By Lemma 8.4(b), $I$ is special, so $W_{I}, W^{\prime} \in \mathscr{W}$. We have $w v \in \mathbb{R}_{\geq 0} \Pi_{I} \cap$ $-\mathscr{C} \subseteq \mathbb{R}_{\geq 0} \Pi_{I} \cap-\mathscr{C}_{I}=\mathscr{K}_{I}$. Since $\Pi_{I}$ is the facial support of $w v$, we get $w v \in \mathscr{Z}_{I}^{\circ}$ by Lemma 10.7 and so $v \in w^{-1} \mathscr{Z}_{I}^{\circ}=\mathscr{Z}_{W^{\prime}}^{\circ}$ as required.

11.4. The next result describes the minimal face of $\mathscr{Z}$ containing $\mathscr{Z}_{W^{\prime}}$ for a reflection subgroup $W^{\prime}$, and also the facial closure of $W^{\prime}$ in terms of this minimal face and extra data determined from the root systems.

Proposition. Let $W^{\prime}$ be a finitely generated reflection subgroup of $W$. Let $W^{\prime \prime}$ be the reflection subgroup of $W^{\prime}$ such that $\Pi_{W^{\prime \prime}}$ is the union of all infinite type components of $\Pi_{W^{\prime}}$. Let $U^{\prime \prime \prime}$ be the reflection subgroup of $W^{\prime \prime}$ with $\Pi_{U^{\prime \prime \prime}}=\Pi_{W^{\prime}} \backslash$ $\Pi_{W^{\prime \prime}}$ i.e. the components of $\Pi_{U^{\prime \prime \prime}}$ are the finite type components of $\Pi_{W^{\prime}}$.

(a) Let $p \in \mathscr{Z}_{W^{\prime}}^{\circ}$. Choose $U^{\prime} \in \mathscr{W}$, so that $p \in \mathscr{Z}_{U^{\prime}}^{\circ}$. Then $U^{\prime}$ is the facial closure of $W^{\prime \prime}$, and $\mathscr{Z}_{U^{\prime}}$ is the minimum face of $\mathscr{Z}$ containing $\mathscr{Z}_{W^{\prime}}$.

(b) $\mathscr{Z}_{W^{\prime}}^{\circ} \subseteq \mathscr{Z}_{U^{\prime}}^{\circ}$

(c) Every component of $\Pi_{U^{\prime \prime \prime}}$ is of finite type, and is either contained in $\mathbb{R} \Pi_{U^{\prime}}$ or is orthogonal to $\mathbb{R} \Pi_{U^{\prime}}$.

(d) Let $\Delta:=\Pi_{U^{\prime \prime \prime}} \cap \Pi_{U^{\prime}}^{\perp}$. Then $\Delta=\Pi_{W^{\prime}} \cap \Pi_{U^{\prime}}^{\perp}$ and $\Delta$ is the union of the (finite type) components of $\Pi_{W^{\prime}}$ which are not contained in $\mathbb{R}_{U^{\prime}}$.

(e) Let $U^{\prime \prime}$ denote the facial (equivalently, parabolic) closure of $W_{\Delta}$. Then $\Phi_{U^{\prime \prime}}=\Phi \cap \mathbb{R} \Delta \subseteq \Pi_{U^{\prime}}^{\perp}$ and the facial closure $U$ of $W^{\prime}$ satisfies $\Pi_{U}=$ $\Pi_{U^{\prime}} \cup \dot{ } \Pi_{U^{\prime \prime}}$. In particular, the facial closures of $W^{\prime}$ and $W^{\prime \prime}$ both have the same infinite type components.

(f) Write $U^{\prime}=w W_{I} w^{-1}$ where $I \subseteq S$ is facial, $w \in W$ and, without loss of generality, $N\left(w^{-1}\right) \cap W_{I \cup I^{\perp}}=\emptyset$. Then $w^{-1} \Delta \subseteq \Phi_{I^{\perp}}$.

(g) We may choose $x \in W_{I}^{\perp}$ and $J \subseteq I^{\perp}$, so that the facial closure of $w^{-1} W_{\Delta} w$ is $x W_{J} x^{-1}$ where without loss of generality, $N\left(x^{-1}\right) \cap J=\emptyset$. Then $I, J$ and $I \cup J$ are facial, and

$$
U^{\prime \prime}=w x W_{J}(w x)^{-1}, \quad U^{\prime}=(w x) W_{I}(w x)^{-1}, \quad U=(w x) W_{I \cup J}(w x)^{-1} .
$$

Remarks. The above leads to an $\mathbb{R}$-algorithm for computing $U, U^{\prime}$ and $U^{\prime \prime}$ from $\chi\left(W^{\prime}\right)$ as follows. First, compute $p \in \mathscr{Z}_{W^{\prime}}^{\circ}$ by 11.2 . Compute $U^{\prime}$ as in (a) and express $U^{\prime}=w^{\prime} W_{I} w^{\prime-1}$ where $I \subseteq S$ is facial and $w \in W$, using 11.3 . Compute the minimal length element $w^{-1}$ of $W_{I \cup I^{\perp}} w^{\prime-1}$; then $U^{\prime}=w W_{I} w^{-1}$ as in (f). Compute $\Delta=\Pi_{W^{\prime}} \cap \Pi_{U^{\prime}}^{\perp}$ as in (d). Since $w^{-1} W_{\Delta} w$ is a finite reflection subgroup of $W_{I^{\perp}}$, one may by 11.2 compute $x^{\prime} \in W_{I^{\perp}}$ and $J \subseteq I^{\perp}$ with the parabolic closure of $w^{-1} W_{\Delta} w$ equal to $x^{\prime} W_{J} x^{-1}$. Let $x^{-1} \in W_{I^{\perp}}$ be the minimal length element of $W_{J} x^{\prime-1}$. Then $x^{\prime} W_{J} x^{\prime-1}=x W_{J} x^{-1}$ is the facial closure of $w^{-1} W_{\Delta} w$ as in $(\mathrm{g})$, since parabolic and facial closures of finite (reflection) subgroups coincide by 8.5(a). Let $z^{-1}$ be the minimal length element of $W_{I \cup J}(w x)^{-1}$. Then $\chi\left(U^{\prime \prime}\right)=z I z^{-1}, \chi\left(U^{\prime}\right)=z J z^{-1}$ and $\chi(U)=z(I \cup J) z^{-1}$ using that $I, J$ are separated.

Proof. Let $\mathscr{Z}_{X}$, with $X \in \mathscr{W}$, denote the inclusion-minimal face of $\mathscr{Z}$ containing $\mathscr{Z}_{W^{\prime}}=\mathscr{Z}_{W^{\prime \prime}}$. By Lemma A.3. (b) holds. It follows that $U^{\prime}=X$, where $U^{\prime}$ is as defined in (a), and (b) holds.

To complete the proof of (a), it remains to show that $U^{\prime}$ is equal to the facial closure $W^{\prime \prime \prime}$ of $W^{\prime \prime}$. Certainly $\mathscr{Z}_{W^{\prime}}=\mathscr{Z}_{W^{\prime \prime}} \subseteq \mathscr{Z}_{W^{\prime \prime \prime}}$. We have $W^{\prime \prime \prime} \in \mathscr{W}$ by 10.1 (e) and we conclude that $U^{\prime}=X \subseteq W^{\prime \prime \prime}$ by definition of $X$. Conversely, let us prove that $W^{\prime \prime \prime} \subseteq U^{\prime}$. Since $U^{\prime}$ is facial, it will suffice to show that $W^{\prime \prime} \subseteq U^{\prime}$. Write $U^{\prime}=w W_{I} w^{-1}$ as in (f). Let $p \in \mathscr{Z}_{W^{\prime}}^{\circ}=\mathscr{Z}_{W^{\prime \prime}}^{\circ}$. Since $W^{\prime \prime}$ has no finite 
type component, Lemma 10.7 implies that $w^{-1} p=\sum_{\alpha \in \Pi_{w}-1_{W^{\prime \prime} w}} c_{\alpha} \alpha$ where all $c_{\alpha}>0$. But by (b), $w^{-1} p \in \mathscr{Z}_{w^{-1} U^{\prime} w}=\mathscr{Z}_{W_{I}}^{\circ} \subseteq \mathbb{R}_{\geq 0} \Pi_{I}$. Since $\Pi_{I}$ is facial, we have $\Pi_{w^{-1} W^{\prime \prime} w} \subseteq \mathbb{R} \Pi_{I} \cap \Phi=\Phi_{I}$. Hence $w^{-1} W^{\prime \prime} w \subseteq W_{I}$ and $W^{\prime \prime} \subseteq w W_{i} w^{-1}=U^{\prime}$. This completes the proof of (a).

Next we prove (c). Let $w, I$ be as in (f). Since $U^{\prime \prime \prime}$ centralizes $W^{\prime \prime}$, it normalizes the facial closure $U^{\prime}$ of $W^{\prime \prime}$. Therefore $w^{-1} U^{\prime \prime \prime} w$ normalizes $w^{-1} U^{\prime} w=W_{I}$. Note that since all components of $\Pi_{I}$ are of infinite type (since those of $\Pi_{U^{\prime}}$ are), 1.15 implies that the normalizer of $W_{I}$ is $W_{I} W_{I^{\perp}}$ (cf. Remark 1.15 . Hence $w^{-1} U^{\prime \prime \prime} w \subseteq$ $W_{I} W_{I^{\perp}}$. Every root of, and hence every irreducible component of, $\Pi_{w^{-1} U^{\prime \prime \prime} w}$ is contained therefore in either $\Phi_{I}$ or $\Phi_{I^{\perp}}$, since $I$ and $I^{\perp}$ are separated. Since $U^{\prime}=$ $w W_{I} w^{-1}$, every irreducible component of $\Pi_{U^{\prime \prime \prime}}$ is contained in either $\mathbb{R}_{U^{\prime}}$ or $\Pi_{U^{\prime}}^{\perp}$, proving (c). Then (d) is clear since every component of $\Pi_{W^{\prime}}$ is either of infinite type (hence contained in $\mathbb{R} \Pi_{W^{\prime \prime}} \subseteq \mathbb{R} \Pi_{U^{\prime}}$ ) or is a component of $\Pi_{U^{\prime \prime \prime}}$. Part (f) is also clear since from the above since $w^{-1} \Delta \subseteq \Phi_{I \cup I^{\perp}}=\Phi_{I} \cup \Phi_{I^{\perp}}$, and $w^{-1} \Delta \subseteq w^{-1} \Pi_{U^{\prime}}^{\perp} \subseteq \Pi_{I}^{\perp}$ implies $w^{-1} \Delta \cap \Phi_{I}=\emptyset$.

Since $w^{-1} W_{\Delta} w$ is finite, its facial closure coincides (by 8.5 (a)) with its parabolic closure, which is clearly contained in $W_{I^{\perp}}$ and so may be written as in $(\mathrm{g})$. We have already seen $I$ is facial. Then $J$ is facial and $I \cup J$ is facial by 8.5, since $W_{J}$ is finite. The formulae for $U^{\prime}$ in (g) holds since $x$ centralizes $W_{I}$. The formula for the facial closure $U^{\prime \prime}$ of $W_{\Delta}$ in (g) follows since $w^{-1} U^{\prime \prime} w$ is the facial closure of $w^{-1} W_{\Delta} w$. Let us now prove the formula $U=(w x) W_{I \cup J}(w x)^{-1}$ from (g). We have

$$
\Pi_{W^{\prime}}=\Pi_{W^{\prime \prime}} \cup \Pi_{U^{\prime \prime \prime}} \subseteq \Phi_{U^{\prime}} \cup \Pi_{U^{\prime \prime \prime}}=\Phi_{U^{\prime}} \cup \Delta \text {. }
$$

Hence

$$
x^{-1} w^{-1} \Pi_{W^{\prime}} \subseteq x^{-1} w^{-1} \Phi_{U^{\prime}} \cup x^{-1} w^{-1} \Delta \subseteq x^{-1} \Phi_{I} \cup \Phi_{J} \subseteq \Phi_{I \cup J} .
$$

In turn this implies that $W^{\prime} \subseteq w x W_{I \cup J}(w x)^{-1}$, so $U \subseteq w x W_{I \cup J}(w x)^{-1}$. On the other hand, $\Phi_{U} \supseteq \Phi_{W^{\prime}} \supseteq \Phi_{W^{\prime \prime}}$ and $U$ facial implies $\Phi_{U} \supseteq \Phi_{U^{\prime}}$. Further, $\Phi_{U} \supseteq \Phi_{W^{\prime}} \supseteq \Phi_{U^{\prime \prime \prime}} \supseteq \Delta$ implies that $\Phi_{U} \supseteq(\mathbb{R} \Delta \cap \Phi)$ by Corollary 2.5 since $U$ is facial. We have $\mathbb{R} w^{-1} \Delta \cap \Phi=\mathbb{R} x \Pi_{J} \cap \Phi$ from Lemma 2.9.(c) and the definition of $J$. Hence

$$
(w x)^{-1} \Phi_{U} \supseteq(w x)^{-1}\left(\Phi_{U^{\prime}} \cup(\mathbb{R} \Delta \cap \Phi)\right) \supseteq x^{-1} \Phi_{I} \cup\left(\mathbb{R}_{J} \cap \Phi\right) \supseteq \Phi_{I} \cup \Phi_{J}=\Phi_{I \cup J}
$$

since $J \subseteq I^{\perp}$ and so $U \supseteq w x W_{I \cup J}(w x)^{-1}$. This proves the formula for $U$ in (g). Finally, (e) follows from the formula for $U^{\prime \prime}, U^{\prime}$ and $U$ in (g) noting $I$ and $J$ are separated.

11.5. The above subsection provides a geometric algorithm for calculating the facial closure of a finitely generated reflection subgroup. This subsection gives an alternative, more algebraic algorithm for determining the facial closure of an arbitrary finitely generated subgroup $W^{\prime}$ of $W$.

First, let $X$ be a finite set of generators for the subgroup $W^{\prime}$. Let $w W_{J} w^{-1}$ denote the parabolic closure of $X$; suitable $w \in W$ and $J \subseteq S$ may be computed by an $\mathbb{R}$-algorithm described in [20]. Using 1.15, one may compute all finitely many standard parabolic subgroups, say $W_{K_{i}}$, for $i=1, \ldots, n$, which are conjugate to $W_{J}$ and determine $x_{i} \in W$ with $K_{i}=x_{i} J x_{i}^{-1}$. Amongst all the standard facial subgroups of $W$ containing any of the sets $K_{i}$, choose one of minimal rank; say $L \subseteq S$ is facial $L \supseteq K_{j}$, and $|L| \leq\left|L^{\prime}\right|$ for any facial $L^{\prime} \subseteq S$ with $L^{\prime} \supseteq K_{i}$ 
for some $i$. Then we claim that the facial closure $W^{\prime \prime}$ of $W^{\prime}$ is $W^{\prime \prime}=U$ where $U:=w x_{j}^{-1} W_{L} x_{j} w^{-1}$.

To see this, note first that $U$ is a facial subgroup of $W$ containing $W^{\prime}$. Write $W^{\prime \prime}=y W_{M} y^{-1}$ for some facial $M \subseteq S$ and some $y \in W$. By Lemma 10.1(d), it will suffice to show that the ranks $|L|$ of $U$ and $|M|$ of $W^{\prime \prime}$ satisfy $|L| \leq|M|$. Now since $W^{\prime \prime}$ is parabolic and contains $W^{\prime}$, we have $W^{\prime \prime} \supseteq w W_{J} w^{-1}$. Then $W_{M} \supseteq y^{-1} w W_{J} w^{-1} y$. This implies that the right hand side is a parabolic subgroup of $W_{M}$, so it is conjugate in $W_{M}$ to some standard parabolic subgroup of $W_{M}$; this standard parabolic is one of $W_{K_{1}}, \ldots, W_{K_{n}}$, say $W_{K_{i}}$, since it is $W$-conjugate to $W_{J}$. Since $M \supseteq K_{i}$, we have $|M| \geq|L|$ as required, by choice of $L$. We conclude that $W^{\prime \prime}=U$ as claimed.

Remarks. The facial closure of a subgroup $W^{\prime}=\langle X\rangle$ of $W$, where $X \subseteq W$ is not necessarily finite, is the parabolic subgroup of maximal rank (necessarily bounded by $|S|$ ) amongst the facial closures of subgroups $\left\langle X^{\prime}\right\rangle$ of $W^{\prime}$ such that $X^{\prime} \subseteq X$ is finite. In case $W^{\prime}$ is a reflection subgroup, this can be made more precise as follows. Let $W^{\prime \prime}$ be a finitely-generated reflection subgroup of $W^{\prime}$ such that $\mathbb{R} \Pi_{W^{\prime \prime}}=\mathbb{R} \Pi_{W^{\prime}}$ (which is possible since $V$ is finite dimensional). Then the facial closure $U$ of $W^{\prime \prime}$ is equal to the facial closure $U^{\prime}$ of $W^{\prime}$. For obviously $U^{\prime} \supseteq U$. On the other hand, we have by 2.5 since $U$ is facial that

$$
\Phi_{U}=\mathbb{R} \Phi_{U} \cap \Phi \supseteq \mathbb{R} \Phi_{W^{\prime \prime}} \cap \Phi=\mathbb{R} \Phi_{W^{\prime}} \cap \Phi \supseteq \Phi_{W^{\prime}}
$$

so $U \supseteq W^{\prime}$ and hence $U \supseteq U^{\prime}$.

\section{The IMAGINARY CONE IN GENERAL}

12.1. In this section, $(W, S)$ is a general Coxeter system realized as in 1.3 .

We first make some general comments about the relation of this general situation to the more special case in which the additional assumptions 4.1(i)-(iii) hold.

An extension or restriction of quadratic space of a based root system, in the sense of Remark 1.3 , does not change the abstract group $W$, or the roots $\Phi$, simple roots $\Pi$, or cones $\mathscr{Y}, \mathscr{Z}$ or $\mathscr{K}$, (as subsets of $\mathbb{R} \Pi$ ) though in general it may change $\mathscr{X}, \mathscr{X}^{*}$, the set facial subsets of $S$ etc. Recall also that any based root system $(\Phi, \Pi)$ on $(V,\langle-,-\rangle)$ has ample extensions in the sense of Remark $1.3(2)$; these may even be chosen so the extended quadratic space is non-singular (and finitedimensional if $V$ is finite-dimensional). On the other hand, if the form $\langle-,-\rangle$ on $V$ is non-singular, then any finite dimensional subspace of $V$ is contained in a finite dimensional subspace $U^{\prime}$ such that the restriction of the form $\langle-,-\rangle$ to $U$ is non-singular. Then $V=U^{\prime} \oplus U^{\prime \perp}$.

These remarks imply that for the study of $\mathscr{Z}$, there is no loss of generality in assuming that the quadratic space is non-singular and ample. Further, if $\Pi$ is finite, there is no loss of generality for studying $\mathscr{Z}$ in assuming 4.1 (i)-(iii).

12.2. With the above remarks in hand, we show Theorem 6.3 still holds under the more relaxed assumptions here.

Theorem. (a) $\mathscr{Z}:=\bigcup_{W}, \mathscr{Z}_{W}$ where in the union, $W^{\prime}$ ranges over any cofinal subfamily of the inclusion-ordered family of all finitely generated reflection subgroups of $W$.

(b) For any reflection subgroup $W^{\prime}$ of $W$, we have $\mathscr{Z}_{W^{\prime}} \subseteq \mathscr{Z}$. 
Proof. Observe that $\mathscr{Z}_{W^{\prime}} \subseteq \mathscr{Z}_{W^{\prime \prime}}$ for any finitely generated reflection subgroups $W^{\prime} \subseteq W^{\prime \prime}$ of $W$, by reducing to the case in which 4.1)(i) - (iii) hold using the above remarks and using Theorem 6.3

Write the family of reflection subgroups in (a) as $W_{j}^{\prime}$ for $j$ in an index set $J$, and let $G$ be the family of finite subsets of $S$. For each $j \in J$, there is some finite $I_{j} \in G$ with $W_{j}^{\prime} \subseteq W_{I_{j}}$, since $W_{j}^{\prime}$ is finitely generated. Also, since $\left\{W_{j}^{\prime}\right\}_{j \in J}$ is cofinal in the family of finitely generated reflection subgroups, there is for each $I \in G$ there is some $j_{I} \in J$ with $W_{I} \subseteq W_{j_{I}}^{\prime}$. By the above remarks, we have $\mathscr{Z}_{j} \subseteq \mathscr{Z}_{I_{j}}$ for $j \in J$ and $\mathscr{Z}_{I} \subseteq \mathscr{Z}_{W_{j_{I}}}$ for $I \in G$. Hence

$$
\cup_{j \in J} \mathscr{Z}_{W_{j}^{\prime}}=\cup_{I \in G} \mathscr{Z}_{I}=\mathscr{Z}
$$

by Lemma 3.3 proving (a).

To prove (b), let $H$ be the family of all finitely generated reflection subgroups of $W$. By (a) applied successively to $W^{\prime}$ and $W$, we have that

$$
\mathscr{Z}_{W^{\prime}}=\bigcup_{\substack{W^{\prime \prime} \in H \\ W^{\prime \prime} \subseteq W^{\prime}}} \mathscr{Z}_{W^{\prime \prime}} \subseteq \bigcup_{W^{\prime \prime} \in H} \mathscr{Z}_{W^{\prime \prime}}=\mathscr{Z}
$$

as required.

12.3. We have not investigated the facial structure of $\mathscr{Z}$ in general. However, as a corollary of the results of this section, the relative interior of $\mathscr{Z}$ may be described under more general assumptions than those of Sections 411.

Corollary. Let $W^{\prime}$ be a subgroup of $W$ such that $\mathbb{R} \mathscr{Z}_{W^{\prime}}$ is finite dimensional. Let $G$ be the family of all finitely generated reflection subgroups $W^{\prime \prime}$ of $W$ such that $\mathbb{R} \mathscr{Z}_{W^{\prime \prime}}=\mathbb{R} \mathscr{Z}_{W^{\prime}}$. Let $Y:=\cup_{W^{\prime \prime} \in G} \mathscr{Z}_{W^{\prime \prime}}^{\circ}$. Then

(a) $G$ is cofinal in the family of all finite rank reflection subgroups of $W^{\prime}$.

(b) $\mathscr{Z}_{W^{\prime}}^{\circ}=Y$.

Proof. By Theorem 12.2, we have $\mathscr{Z}_{W^{\prime}}=\cup_{W^{\prime \prime} \subseteq W^{\prime}} \mathscr{Z}_{W^{\prime \prime}}$ where the union is over all finite rank reflection subgroups $W^{\prime \prime}$ of $W^{\prime}$. From this and Theorem 12.2, one gets (a). For $W^{\prime \prime} \in G, \mathscr{Z}_{W}^{\circ}$ is an open subset of $\mathbb{R} \mathscr{Z}_{W^{\prime \prime}}=\mathbb{R} \mathscr{Z}_{W^{\prime}}$ and is contained in $\mathscr{Z}_{W^{\prime}}$. Hence $Y$ is an open subset of $\mathbb{R} \mathscr{Z}_{W^{\prime}}$ contained in $\mathscr{Z}_{W^{\prime}}$ and it follows that $Y \subseteq \mathscr{Z}_{W^{\prime}}^{\circ}$. For the reverse inclusion, let $\alpha \in \mathscr{Z}_{W^{\prime}}^{\circ}$. Then there is a (finite) basis $\Gamma$ of $\mathbb{R} \mathscr{Z}_{W^{\prime}}$ such that $\Gamma \subseteq \mathscr{Z}_{W^{\prime}}$ and $\alpha \in \mathbb{R}_{>0} \Gamma$. Using (a) and Theorem 12.2, there is some $W^{\prime \prime} \in G$ such that $\Gamma \subseteq \mathscr{Z}_{W^{\prime \prime}}$. Then $\alpha \in \mathbb{R}_{>0} \Gamma \subseteq \mathscr{Z}_{W^{\prime \prime}}^{\circ} \subseteq Y$, completing the proof.

12.4. Finally, we return to the assumptions of Sections 411 and describe the minimal face of $\mathscr{Z}_{W}$ containing $\mathscr{Z}_{W^{\prime}}$ for an arbitrary (not necessarily finite rank) reflection subgroup $W^{\prime}$ of $W$.

Corollary. Assume that the conditions 4.1(i)-(iii) hold. Let $W^{\prime}$ be an arbitrary reflection subgroup of $W$ and let $U$ denote the facial closure of $W^{\prime}$.

(a) $\mathscr{Z}_{U}$ is the minimum face of $\mathscr{Z}$ which contains $\mathscr{Z}_{W^{\prime}}$.

(b) $\mathscr{Z}_{W}^{\circ} \subseteq \mathscr{Z}_{U}^{\circ}$.

Proof. Suppose first that $W^{\prime}$ is finitely generated. Let $W^{\prime \prime}, U^{\prime}, U^{\prime \prime}$ be as in 11.4 . By 11.4 $\mathscr{Z}_{U^{\prime}}=\mathscr{Z}_{U}$ is the minimal face of $\mathscr{Z}$ containing $\mathscr{Z}_{W^{\prime \prime}}=\mathscr{Z}_{W^{\prime}}$, and $\mathscr{Z}_{W^{\prime}}^{\circ} \subseteq \mathscr{Z}_{U}^{\circ}$.

In general, note that the family $G^{\prime}$ of finite rank reflection subgroups $W^{\prime \prime \prime}$ of $W^{\prime}$ with $\mathbb{R} \Pi_{W^{\prime \prime \prime}}=\mathbb{R} \Pi_{W^{\prime}}$ and $\mathbb{R} \mathscr{Z}_{W^{\prime \prime \prime}}=\mathbb{R} \mathscr{Z}_{W^{\prime}}$ is cofinal in the family of all finite 
rank reflections subgroups of $W$. Any $W^{\prime \prime \prime} \in G^{\prime}$ has $U$ as facial closure by Remark 11.5 and $\mathscr{Z}_{W^{\prime \prime \prime}} \subseteq \mathscr{Z}_{U}$. Both (a)-(b) now follow easily from the special case in the previous paragraph, using that $\mathscr{Z}_{W^{\prime}}=\cup_{W^{\prime \prime \prime} \in G^{\prime}} \mathscr{Z}_{W^{\prime \prime \prime}}$ by Theorem 12.2 and $\mathscr{Z}_{W^{\prime}}^{\circ}=\cup_{W^{\prime \prime \prime} \in G^{\prime}} \mathscr{Z}_{W^{\prime \prime \prime}}^{\circ}$ by Corollary 12.3 .

\section{Appendix A. Facial structure of cones}

This section discusses (largely without proof) some mostly standard facts concerning purely algebraic aspects of facial structure of cones, which are used in this paper or may be helpful in understanding the main results. These facts can be found scattered in various sources between which there is not always agreement on terminology and conventions, and for the readers convenience, we record them here in a uniform way. Though, strictly, these results are only applied in this paper in finite dimensional spaces, so the reader may consider such spaces if desired, we discuss many of them them more generally to clarify the role played by finite dimensionality and since the general versions may be useful in possible extensions of this work. As references for this material, see [5] and [1] for general real vector spaces, and, for finite dimensional spaces, [44, [51, 44], 23, 8 8 and 27. For background on partially ordered sets, lattices, directed sets, Galois connections etc which is also used here and elsewhere in the paper, see for instance [10. In this paper, the only Galois connections which occur are those between power sets $\mathscr{P}(X)$ and $\mathscr{P}(Y)$, associated to a relation $R \subseteq X \times Y$ as in [10, 7.22].

A.1. In this section, all vector spaces are endowed with a standard topology, which we call the finest locally convex topology (flc topology, for short) defined below. Though some of the properties stated in the following subsections hold for all locally convex (Hausdorff) vector topologies (see 5 for details), many do not.

A subset $C$ of a real affine space $V$ is said to be algebraically open if for all affine lines $l$ of $V$, the intersection $l \cap C$ is an open subset of $l$ (in the topology obtained by transferring the standard topology on $\mathbb{R}$ to $l$ by an affine isomorphism $\mathbb{R} \rightarrow l$ ). The flc topology on $V$ is the (Hausdorff) topology on $V$ so the open subsets are the unions of algebraically open convex subsets of $V$. The flc topology on $V$ is clearly invariant under all affine automorphisms of $V$. It is easily seen (1, (3.3), Problem 4]) that if $V$ is a vector space, the flc topology is the finest topology making $V$ a locally convex topological vector space. (By [5, Ch II, $\S 4$, no. 2, 2], the flc topology coincides with the topology determined by the family of all seminorms on $V$.) Other easily checked properties of flc topology are as follows. If $V$ is finite dimensional, the flc topology is the standard topology on $V$ (defined for example by the norm associated to a positive definite inner product on $V$ ). In general, any affine subset $U$ of $V$ is closed (hence each affine function $V \rightarrow \mathbb{R}$ is continuous) and (if $U \neq \emptyset$ ) the subspace and flc topology on $U$ coincide. The flc topology on a product $V_{1} \times V_{2}$ of real affine spaces $V_{1}, V_{2}$ is the product of the flc topologies on $V_{1}$ and $V_{2}$. See [1], [5. Ch II] for more details and further properties.

A.2. In the remainder of this section, let $V$ be a real vector space, in its flc topology. Fix a convex set $\mathscr{Y} \subseteq V$. A point $v$ of $\mathscr{Y}$ is called an algebraically interior point of $\mathscr{Y}$ in $V$ if for each affine line $l$ of $V$ with $v \in l$, the point $v$ is in the interior (with respect to $l$ ) of $l \cap \mathscr{Y}$ (see [1, Ch II, (1.5)]). It is easily shown that $\operatorname{ri}(\mathscr{Y})$ is the set of all algebraically interior points of $\mathscr{Y}$ in aff(Y) . Using [5, Ch II, 2 , no. 6, 
Proposition 16 and Corollaire 1] together with $\operatorname{cl}(\mathscr{Y}) \subseteq$ aff( $\mathscr{Y})$ (which holds since we use flc topology) shows

$$
\begin{aligned}
& \text { if } x \in \operatorname{ri}(\mathscr{Y}) \text { and } y \in \overline{\mathscr{Y}} \text {, then } t x+(1-t) y \in \operatorname{ri}(\mathscr{Y}) \text { for } 0<t<1, \\
& \quad \text { if } \operatorname{ri}(\mathscr{Y}) \neq \emptyset \text {, then } \overline{\operatorname{ri}(\mathscr{Y})}=\overline{\mathscr{Y}} \text { and } \operatorname{ri}(\overline{\mathscr{Y}})=\operatorname{ri}(\mathscr{Y}) .
\end{aligned}
$$

(For examples of non-empty cones without any relative interior points in the flc topology, see [1, Ch II, (1.4)] and [27, Proposition 11.1]).

A.3. A subset $\mathscr{C}$ of $\mathscr{Y}$ is said to be a face or an extreme subset of $\mathscr{Y}$ if it is convex and for all $c \in \mathscr{C}, y_{1}, y_{2} \in \mathscr{Y}$ and $t \in(0,1)$ with $c=t y_{1}+(1-t) y_{2}$, one has $y_{1}, y_{2} \in \mathscr{C}$. See [1] for general background on faces. A face $\mathscr{C}$ of $\mathscr{Y}$ is proper if $\mathscr{C} \neq \mathscr{Y}$. Any intersection or directed union of faces of $\mathscr{Y}$ is a face of $\mathscr{Y}$, and a face of a face of $\mathscr{Y}$ is an face of $\mathscr{Y}$. In particular, the set of faces of $\mathscr{Y}$, ordered by inclusion, forms a complete lattice $\operatorname{Ext}(\mathscr{Y})$, called here the face lattice of $\mathscr{Y}$. The proof of [44, Theorem 18.1] shows

(A.3.1) if $\mathscr{C} \in \operatorname{Ext}(\mathscr{Y}), \mathscr{Z} \subseteq \mathscr{Y}, \mathscr{Z}$ is convex and $\mathscr{C} \cap \operatorname{ri}(\mathscr{Z}) \neq \emptyset$, then $\mathscr{Z} \subseteq \mathscr{C}$.

Combining the proofs of [47, (2.4.4)] and [51, Theorem 2.6.2] shows that if $\mathscr{C} \subseteq \mathscr{Y}$,

$$
\mathscr{C} \in \operatorname{Ext}(\mathscr{Y}) \Longleftrightarrow(\mathscr{Y} \backslash \mathscr{C} \text { is convex and } \mathscr{C}=\operatorname{aff}(\mathscr{C}) \cap \mathscr{Y}) .
$$

Since $\operatorname{aff}(\mathscr{C})$ is closed in $V$, it follows that any face of $\mathscr{Y}$ is closed in $\mathscr{Y}$.

For any $\mathscr{K} \subseteq \mathscr{Y}$, there is an inclusion minimal face $\mathscr{Y}_{\mathscr{K}}$ of $\mathscr{Y}$ containing $\mathscr{K}$, namely the intersection of all faces of $\mathscr{Y}$ which contain $\mathscr{K}$. If also $\mathscr{K}^{\prime} \subseteq \mathscr{Y}$, one clearly has $\mathscr{Y}_{\mathscr{K} \cup \mathscr{K}^{\prime}}=\mathscr{Y}_{\mathscr{K}} \vee \mathscr{Y}_{\mathscr{K}^{\prime}}$ where $\vee$ denotes join in $\operatorname{Ext}(\mathscr{Y})$. For $y \in \mathscr{Y}$ let $\mathscr{Y}_{y}:=\mathscr{Y}_{\{y\}}$ and $U_{y}=\operatorname{aff}\left(\mathscr{Y}_{y}\right)$. From A.3.2), $\mathscr{Y}_{y}=\mathscr{Y} \cap U_{y}$ and by $[5$, Ch, $\S 7$, Ex3], $U_{y}$ is the inclusion-largest affine subset $U$ of $V$ containing $\{y\}$ such that $y$ is an algebraically interior point of $U \cap \mathscr{Y}$ in $U$. In particular, $y \in \operatorname{ri}\left(\mathscr{Y}_{y}\right)$. It follows from this and A.3.1 that any convex set $\mathscr{Z} \subseteq \mathscr{Y}$ with a relative interior point $y$ is contained in $\mathscr{Y}_{y}$. Since any face $\mathscr{Z}$ of $\mathscr{Y}$ which contains $y$ is convex and contains $\mathscr{Y}_{y}$, one has

$$
\left\{\mathscr{Y}_{y} \mid y \in \mathscr{Y}\right\}=\{\mathscr{Z} \in \operatorname{Ext}(\mathscr{Y}) \mid \operatorname{ri}(\mathscr{Z}) \neq \emptyset\}
$$

Similarly, for $y, y^{\prime} \in \mathscr{Y}$, one has

$$
\mathscr{Y}_{y^{\prime}}=\mathscr{Y}_{y} \Longleftrightarrow y^{\prime} \in \operatorname{ri}\left(\mathscr{Y}_{y}\right) \Longleftrightarrow \operatorname{ri}\left(\mathscr{Y}_{y^{\prime}}\right)=\operatorname{ri}\left(\mathscr{Y}_{y}\right)
$$

and

$$
\operatorname{ri}\left(\mathscr{Y}_{y^{\prime}}\right) \cap \operatorname{rb}\left(\mathscr{Y}_{y}\right) \neq \emptyset \Longleftrightarrow \mathscr{Y}_{y^{\prime}} \subseteq \operatorname{rb}\left(\mathscr{Y}_{y}\right) \Longleftrightarrow \mathscr{Y}_{y^{\prime}} \subsetneq \mathscr{Y}_{y} .
$$

Hence

(A.3.6) $\left\{\operatorname{ri}\left(\mathscr{Y}_{y}\right) \mid y \in \mathscr{Y}\right\}$ is a partition of $\mathscr{Y}$ into relatively open convex subsets.

and

$$
\mathscr{Y} \cap(\operatorname{rb} \mathscr{Y})=\bigcup_{\substack{\mathscr{C} \in \operatorname{Ext}(\mathscr{Y}) \\ \mathscr{C} \neq \mathscr{Y}}} \mathscr{C}=\bigcup_{\substack{\mathscr{C} \in \operatorname{Ext}(\mathscr{Y}) \\ \mathscr{C} \neq \mathscr{Y}}} \operatorname{ri}(\mathscr{C}) .
$$

Note that

$$
\mathscr{C} \in \operatorname{Ext}(\mathscr{Y}) \Longrightarrow \mathscr{C}=\bigcup_{y \in \mathscr{C}} \mathscr{Y}_{y} \text { (directed union) }
$$


since for $y \in \mathscr{C}, \mathscr{Y}_{y} \subseteq \mathscr{C}$ and for all $y, y^{\prime} \in \mathscr{C}$, one has $\mathscr{Y}_{y} \vee \mathscr{Y}_{y^{\prime}}=\mathscr{Y}_{\left\{y, y^{\prime}\right\}}=\mathscr{Y}_{y^{\prime \prime}}$ where $y^{\prime \prime}:=\frac{1}{2} y+\frac{1}{2} y^{\prime} \in \mathscr{C}$. Recall that $\mathscr{F} \in \operatorname{Ext}(\mathscr{Y})$ is said to be lattice-compact if whenever $\mathscr{F} \subseteq \bigvee_{\mathscr{G} \in I} \mathscr{G}$ where $I \subseteq \operatorname{Ext}(\mathscr{Y})$, one has $\mathscr{F} \subseteq \bigvee_{\mathscr{G} \in I^{\prime}} \mathscr{G}$ for some finite $I^{\prime} \subseteq I$. Using A.3.1 and A.3.8, one easily shows that

(A.3.9) the set of lattice-compact elements of $\operatorname{Ext}(\mathscr{Y})$ is $\left\{\mathscr{Y}_{y} \mid y \in \mathscr{Y}\right\} \cup\{\emptyset\}$.

By definition (see [10]), A.3.8 - A.3.9 show that $\operatorname{Ext}(\mathscr{Y})$ is an algebraic lattice.

Let $U$ be any finite dimensional affine subset of $V$. By A.3.2,

$$
\text { if } C \subseteq \operatorname{Ext}(\mathscr{Y}) \cap \mathscr{P}(U) \text { is totally ordered, then }|C| \leq \operatorname{dim}(U)+2
$$

since $\operatorname{dim}(U)+2$ is the maximum cardinality of a flag of affine subsets of $U$. Let $\mathscr{C} \in \operatorname{Ext}(\mathscr{Y}) \cap \mathscr{P}(U)$. In A.3.8), each $\mathscr{Y}_{y}$ is contained in $\operatorname{Ext}(\mathscr{Y}) \cap \mathscr{P}(U)$. It follows that if $\mathscr{C} \neq \emptyset$, then $\mathscr{C}=\mathscr{Y}_{y}$ for some $y \in \mathscr{C}$ and so ri $(\mathscr{C}) \neq \emptyset$. Taking $U=V$, one recovers the well-known fact (see [44, Theorem 6.2]) that

(A.3.11) if $\operatorname{dim}(V)$ is finite, any convex set $\mathscr{Y} \neq \emptyset$ has a relative interior point.

Then A.3.3 implies that

$$
\text { if } \operatorname{dim}(V) \text { is finite, } \operatorname{Ext}(\mathscr{Y})=\{\emptyset\} \cup\left\{\mathscr{Y}_{y} \mid y \in \mathscr{Y}\right\} .
$$

Two other facts we shall use (see [44, Theorem 6.5, Corollary 6.6.2]) are the following. Let $C_{1}, C_{2}$ be convex subsets of $V$ where $V$ is finite dimensional. Then

$$
\begin{gathered}
\operatorname{ri}\left(C_{1} \cap C_{2}\right)=\operatorname{ri}\left(C_{1}\right) \cap \operatorname{ri}\left(C_{2}\right) \text { if } \operatorname{ri}\left(C_{1}\right) \cap \operatorname{ri}\left(C_{2}\right) \neq 0 \\
\operatorname{ri}\left(C_{1}+C_{2}\right)=\operatorname{ri}\left(C_{1}\right)+\operatorname{ri}\left(C_{2}\right)
\end{gathered}
$$

The proof of the following lemma is left to the reader.

Lemma. Suppose that $\operatorname{dim}(V)$ is finite and $\mathscr{Y} \subseteq V$ is a convex set. Let $C$ be a non-empty convex subset of $\mathscr{Y}$.

(a) The following conditions (i)-(iii) on $F \in \operatorname{Ext}(\mathscr{Y})$ are equivalent:

(i) $C \subseteq F$

(ii) $\operatorname{ri}(C) \subseteq F$.

(iii) $\operatorname{ri}(C) \cap F \neq \emptyset$.

(b) The following conditions (i)-(iv) on $F \in \operatorname{Ext}(\mathscr{Y})$ are equivalent:

(i) $F$ is minimal with $C \subseteq F$.

(ii) $\operatorname{ri}(F) \supseteq \operatorname{ri}(C)$

(iii) $\operatorname{ri}(F) \cap \operatorname{ri}(C) \neq \emptyset$.

(iv) $F$ is maximal with $\operatorname{ri}(F) \cap C \neq \emptyset$.

Moreover, there is a unique $F$ satisfying (i)-(iv).

A.4. Assume now that $\mathscr{Y}$ is a cone, as will be the case in all applications in this paper. Then a subset $\mathscr{C}$ of $\mathscr{Y}$ is a face if and only if it is a cone and for all $c \in \mathscr{C}$ and $y_{1}, y_{2} \in \mathscr{Y}$ with $c=y_{1}+y_{2}$, one has $y_{1}, y_{2} \in \mathscr{C}$ (see [27, Lemma 10.2(a),(c)]). For $\mathscr{C} \in \operatorname{Ext}(\mathscr{Y}), \mathscr{C}$ and $\mathscr{Y} \backslash \mathscr{C}$ are cones and if $\mathscr{C} \neq \emptyset$, then aff $(\mathscr{C})=\operatorname{lin}(\mathscr{C})$. For $\mathscr{K} \subseteq \mathscr{Y}$, one has

$$
\mathscr{Y}_{\mathscr{K}}=\mathscr{Y}_{\text {cone }(\mathscr{K})} \text { and, if } \mathscr{K} \text { is a cone, } \mathscr{Y}_{\mathscr{K}}=(\mathscr{K}-\mathscr{Y}) \cap \mathscr{Y}
$$

as is easily checked (see [27, Lemma $10.2(\mathrm{e})]$ in case $\mathscr{K}=\mathbb{R}_{>0} x$ ). If $0 \in \mathscr{Y}$, then every non-empty face $\mathscr{C}$ of $\mathscr{Y}$ contains 0 , and $\operatorname{Ext}_{\neq \emptyset}(\mathscr{Y}):=\operatorname{Ext}(\mathscr{Y}) \backslash\{\emptyset\}$ is itself 
a complete lattice in which meets and joins of its (possibly infinite) subsets are the same as in $\operatorname{Ext}(\mathscr{C})$. A ray which is a face of $\mathscr{Y}$ is called an extreme ray of $\mathscr{Y}$.

The next result is easily proved from A.2.1 - A.2.2.

Lemma. Suppose $\mathscr{Y} \subseteq V$ is a cone and $\rho \in \operatorname{ri}(\mathscr{Y})$. Then

(a) $\operatorname{cl}(\mathscr{Y})=\left\{z \in V \mid z+t \rho \in \mathscr{Y}\right.$ for all $\left.t \in \mathbb{R}_{>0}\right\}$.

(b) $\operatorname{ri}(\mathscr{Y})=\left\{z+t \rho \mid z \in \operatorname{ri}(\mathscr{Y}), t \in \mathbb{R}_{>0}\right\}=\left\{z+t \rho \mid z \in \mathscr{Y}, t \in \mathbb{R}_{>0}\right\}=$ $\left\{z+t \rho \mid z \in \operatorname{cl}(\mathscr{Y}), t \in \mathbb{R}_{>0}\right\}$.

A.5. For the rest of this section, fix a bilinear map $\langle-,-\rangle: V \times U \rightarrow \mathbb{R}$ where $V$, $U$ are real vector spaces.

In general, if $A \subseteq V, B \subseteq U$, write $\langle A, B\rangle$ for $\{\langle a, b\rangle \mid a \in A, b \in B\}$. Partially order the power sets $\mathscr{P}(V), \mathscr{P}(U)$ by inclusion. Define maps $C \mapsto C^{*}$ and $C \mapsto C^{\perp}$ from $\mathscr{P}(V) \rightarrow \mathscr{P}(U)$ by

$$
C^{*}:=\left\{u \in U \mid\langle C, u\rangle \subseteq \mathbb{R}_{\geq 0}\right\}, \quad C^{\perp}:=\{u \in U \mid\langle C, u\rangle \subseteq\{0\}\}
$$

for each subset $C$ of $V$. By symmetry, define maps $D \mapsto D^{*}$ and $D \mapsto D^{\perp}$ from $\mathscr{P}(U) \rightarrow \mathscr{P}(V)$ (we rely on context rather than a more elaborate notation to distinguish the meaning of $C^{\perp}, C^{*}$, and also $\operatorname{wcl}(C)$ as defined below, if $\left.C \subseteq U \cap V\right)$.

Note $C^{*}$ is a pointed cone in $U$; in fact, $C^{*}$ contains the right radical $V^{\perp}$ of $\langle-,-\rangle$. If $C$ is a cone, $C^{*}$ is called the dual cone of $C$. It is easy to see that the pair of order-reversing maps $C \mapsto C^{*}: \mathscr{P}(V) \rightarrow \mathscr{P}(U)$ and $D \mapsto D^{*}: \mathscr{P}(U) \rightarrow \mathscr{P}(V)$ define a Galois connection between $\mathscr{P}(U)$ and $\mathscr{P}(V)$ (see [10). General properties of Galois connections imply the following. For $C \subseteq V$, one has $C \subseteq C^{* *}$; further, $C=C^{* *}$ if and only if $C=D^{*}$ for some $D \subseteq U$. If $C=C^{* *}, C$ is said to be a stable cone in $V$. The map $C \mapsto C^{*}$ defines an inclusion-reversing bijection from the set of stable cones of $V$ to the set of stable cones of $U$.

Remarks. (1) The weak topology on $V$ is defined to be the weakest topology on $V$ so all linear maps $\langle-, u\rangle: V \rightarrow \mathbb{R}$ for $u \in U$ are continuous (it is Hausdorff if and only if the left radical $U^{\perp}$ of $\langle-,-\rangle$ is zero). Denote the weak closure operator on $V$ as wcl. It follows from the bipolar theorem (see [5. Ch II, S6, no. 3, Théorème 1 and Proposition 4(ii)] or [1, (5.3)]) that for $C \subseteq V$,

$$
C^{* *}=\operatorname{wcl}\left(\mathbb{R}_{\geq 0} C\right)
$$

and that the stable cones in $V$ are the non-empty weakly closed cones in $V$.

(2) Similarly, the maps $\perp$ between $\mathscr{P}(V)$ and $\mathscr{P}(V)$ determine a Galois connection with the weakly closed subspaces of $U$ and $V$ as the stable sets.

A.6. Let $\mathscr{Y}, \mathscr{Z}$ be cones in $V$ and $U$ respectively such that $\langle\mathscr{Y}, \mathscr{Z}\rangle \subseteq \mathbb{R}_{>0}$ i.e. $\mathscr{Z} \subseteq \mathscr{Y}^{*}$ or, equivalently, $\mathscr{Y} \subseteq \mathscr{Z}^{*}$. Such a pair of cones will be called a semidual pair of cones. There is then a transpose semidual pair $P^{\operatorname{tr}}:=(\mathscr{Z}, \mathscr{Y})$ with respect to the bilinear map $\langle-,-\rangle \circ \iota: U \times V \rightarrow \mathbb{R}$ where $\iota: U \times V \rightarrow V \times U$ is the isomorphism $(u, v) \mapsto(v, u)$. If $(\mathscr{Y}, \mathscr{Z})$ is a semidual pair with respect to $\langle-,-\rangle$, so is $\left(\mathscr{Y}, \mathscr{Z}^{* *}\right)$. If $(\mathscr{Y}, \mathscr{Z})$ is a semidual pair such that $\mathscr{Z}=\mathscr{Y}^{*}$ and $\mathscr{Y}=\mathscr{Z}^{*}$, then $(\mathscr{Y}, \mathscr{Z})$ is said to be a semidual pair of stable cones. For any $\mathscr{Y} \subseteq V,\left(\mathscr{Y}^{* *}, \mathscr{Y}^{*}\right)$ is a semidual pair of stable cones.

Assume below that $P=(\mathscr{Y}, \mathscr{Z})$ is a semidual pair of cones. Let $z \in \mathscr{Z}$. Then $z^{*} \supseteq \mathscr{Z}^{*}=\mathscr{Y}^{* *} \supseteq \mathscr{Y}$. If $z^{\perp} \neq V$ and $z^{\perp} \cap \mathscr{Y} \neq \emptyset$, then $z^{*}$ is called a supporting (homogeneous) half-space of $\mathscr{Y}$ and $z^{\perp}=\operatorname{rb}\left(z^{*}\right)$ is called a supporting (linear) hyperplane of $\mathscr{Y}$. Any subset of $\mathscr{Y}$ which is either equal to $\mathscr{Y}$ or of the form 
$\mathscr{Y} \cap z^{\perp}$ for an arbitrary $z \in \mathscr{Z}$ is a face of $\mathscr{Y}$. Such a face will be called an exposed face of $\mathscr{Y}$. Thus the intersections of $\mathscr{Y}$ with its supporting hyperplanes are the proper, non-empty exposed faces of $\mathscr{Y}$, and possibly $\mathscr{Y}$ itself. Note that

$$
\text { if } z, z^{\prime} \in \mathscr{Z},\left(\mathscr{Y} \cap z^{\perp}\right) \cap\left(\mathscr{Y} \cap z^{\perp}\right)=\mathscr{Y} \cap\left(z+z^{\prime}\right)^{\perp} \text { where } z+z^{\prime} \in \mathscr{Z} \text {. }
$$

Hence the set of exposed subsets of $\mathscr{Y}$ forms a meet semilattice $\operatorname{Exp}_{P}(\mathscr{Y})$ with maximum element $\mathscr{Y}$. It is not necessarily a complete meet semilattice.

An arbitrary (possibly empty) intersection of exposed faces of $\mathscr{Y}$ is called a semi-exposed face of $\mathscr{Y}$. A ray $\mathbb{R}_{\geq 0} \alpha$ which is a face (resp., an exposed face, semiexposed face ) of a (pointed) cone $\mathscr{Y}$ is called an extreme ray (resp., exposed ray, semiexposed ray) of $\mathscr{Y}$. The set of all semi-exposed subsets of $\mathscr{Y}$ forms a complete lattice $\operatorname{SExp}_{P}(\mathscr{Y})$ with maximum element $\mathscr{Y}$. Define the order-reversing maps $Y \mapsto Y^{\dagger}: \mathscr{P}(\mathscr{Y}) \rightarrow \mathscr{P}(\mathscr{Z})$ given by $Y^{\dagger}:=\mathscr{Z} \cap Y^{\perp}$ and $Z \rightarrow Z^{\#}: \mathscr{P}(\mathscr{Z}) \rightarrow \mathscr{P}(\mathscr{Y})$ given by $Z^{\#}:=\mathscr{Y} \cap Z^{\perp}$. Since $Y \subseteq Z^{\#}$ if and only if $\langle Y, Z\rangle \subseteq\{0\}$ if and only if $Z \subseteq Y^{\dagger}$, these maps also define a Galois connection. The stable subsets of $\mathscr{Y}$ for this Galois connection are the subsets $Y$ of $\mathscr{Y}$ with $Y=Y^{\dagger \#}$ or, equivalently, with $Y=Z^{\#}$ for some $Z \subseteq \mathscr{Z}$. Note that for all $z \in \mathscr{Z}, z^{\#}=\mathscr{Y} \cap z^{\perp} \in \operatorname{Exp}_{P}(\mathscr{Y})$ is an exposed face of $\mathscr{Y}$ and that for $Z \subseteq \mathscr{Z}, Z^{\#}=\cap_{z \in Z} z^{\#}$. Hence the stable subsets of $\mathscr{Y}$ are precisely its semi-exposed subsets. Similar results hold for $\mathscr{Z}$ by symmetry. Standard properties of Galois connections imply that the maps $Z \mapsto Z$ \# and $Y \mapsto Y^{\dagger}$ restrict to mutually inverse, inclusion-reversing bijections between the stable subsets of $\mathscr{Y}$ and $\mathscr{Z}$ i.e. between $\operatorname{SExp}_{P}(\mathscr{Y})$ and $\operatorname{SExp}_{P}(\mathscr{Z})$.

Any semi-exposed face is a (weakly closed and hence closed in flc topology) face of $\mathscr{Y}$, and it contains 0 if $0 \in \mathscr{Y}$ (since this is readily checked for exposed subsets $z^{\#}=\mathscr{Y} \cap z^{\perp}$, where $z \in \mathscr{Z}$ ). For any $Z \subseteq \mathscr{Z}, Z^{\#}=Z^{\# \dagger \#}=\bigcap_{z \in Z^{\# \dagger}} z^{\#}$ is (by A.6.1 and the fact that $\mathscr{Z}^{\# \dagger}$ is a cone) the intersection of the directed downwards (by inclusion) family of exposed subsets $z^{\#}$ of $\mathscr{Y}$, for $z \in \mathscr{Z} \# \dagger$. Using A.3.10 now shows that if $V$ is finite dimensional, then any semi-exposed face of $\mathscr{Y}$ is exposed. Hence

(A.6.2) if $\operatorname{dim}(V)$ is finite, $\operatorname{Exp}_{P}(\mathscr{Y})=\operatorname{SExp}_{P}(\mathscr{Y})$ is a complete lattice.

For $z \in \mathscr{Z}$, one has $z \subseteq z^{\# \dagger}$ where the right hand side is an exposed subset, and hence in particular $z^{\# \dagger}$ is a weakly closed face of $\mathscr{Z}$. It follows that

$$
z \in \operatorname{ri}\left(\mathscr{Z}_{z}\right) \subseteq \mathscr{Z}_{z} \subseteq \operatorname{cl}\left(\mathscr{Z}_{z}\right) \subseteq \operatorname{wcl}\left(\mathscr{Z}_{z}\right) \subseteq z^{\# \dagger}
$$

Applying the inclusion reversing map \# to this and using $z^{\#}=z^{\# \dagger \#}$ shows that

$$
z^{\#}=\left(\operatorname{ri}\left(\mathscr{Z}_{z}\right)\right)^{\#}=\left(\mathscr{Z}_{z}\right)^{\#}=\left(\operatorname{cl}\left(\mathscr{Z}_{z}\right)\right)^{\#}=\left(\operatorname{wcl}\left(\mathscr{Z}_{z}\right)\right)^{\#} \text {. }
$$

Remarks. Using A.6.4, the above Galois connection between subsets of $\mathscr{Y}$ and $\mathscr{Z}$ can be described in terms of a Galois connection between subsets of the "quotient" sets $\mathscr{Y}^{\prime}:=\left\{\operatorname{ri}\left(\mathscr{Y}_{y}\right) \mid y \in \mathscr{Y}\right\}$ and $\mathscr{Z}^{\prime}:=\left\{\operatorname{ri}\left(\mathscr{Z}_{z}\right) \mid z \in \mathscr{Z}\right\}$ of $\mathscr{Y}, \mathscr{Z}$ (of relative interiors of the non-empty lattice-compact faces of $\mathscr{Y}, \mathscr{Z}$ ) as follows. There are order-reversing maps $f: \mathscr{P}\left(\mathscr{Y}^{\prime}\right) \rightarrow \mathscr{P}\left(\mathscr{Z}^{\prime}\right)$ defined by

$$
f(Y):=\left\{z \in \mathscr{Z}^{\prime} \mid\langle z, y\rangle \subseteq\{0\} \text { for all } y \in Y\right\}
$$

for $Y \subseteq \mathscr{Y}^{\prime}$ and $g: \mathscr{P}\left(\mathscr{Z}^{\prime}\right) \rightarrow \mathscr{P}\left(\mathscr{Y}^{\prime}\right)$ defined analogously by symmetry. It is easy to see that $f$ and $g$ define a Galois connection between $\mathscr{P}\left(\mathscr{Y}^{\prime}\right)$ and $\mathscr{P}\left(\mathscr{Z}^{\prime}\right)$. Define the natural surjection ("quotient map") $\pi: \mathscr{Y} \rightarrow \mathscr{Y}^{\prime}$ by $y \mapsto \operatorname{ri}\left(\mathscr{Y}_{y}\right)$, and define $\rho: \mathscr{Z} \rightarrow \mathscr{Z}^{\prime}$ similarly by $z \mapsto \operatorname{ri}\left(\mathscr{Z}_{z}\right)$. Then for $Y \subseteq \mathscr{Y}$, one has $Y^{\dagger}=\rho^{-1}(f(\pi(Y)))$ and for $Z \subseteq \mathscr{Z}$, one has $Z^{\#}=\pi^{-1}(g(\rho(Z))$. 
A.7. In the specially important case that $U=\operatorname{Hom}_{\mathbb{R}}(V, \mathbb{R})$ is the dual space of $V,\langle-,-\rangle: \mathscr{Y} \times \mathscr{Z} \rightarrow \mathbb{R}$ is the canonical evaluation pairing, and $\mathscr{Z}:=\mathscr{Y}^{*}$ is the dual cone of $\mathscr{Y}$, supporting half-spaces and hyperplanes of $\mathscr{Y}$, and exposed and semi-exposed faces of $\mathscr{Y}$, will be distinguished by the adjective "absolute." These absolute notions are completely determined by $\mathscr{Y}$ as a subset of the vector space $V$ alone. Denote the set of absolutely semi-exposed (resp., absolutely exposed) faces of $\mathscr{Y}$ by $\operatorname{ASExp}(\mathscr{Y})($ resp., $\operatorname{AExp}(\mathscr{Y}))$. Note that for a semidual pair $(\mathscr{Y}, \mathscr{Z})$ associated to an arbitrary bilinear map $\langle-,-\rangle$, one has $\operatorname{AExp}(\mathscr{Y}) \subseteq \operatorname{Exp}(\mathscr{Y})$ and therefore $\operatorname{SExp}(\mathscr{Y}) \subseteq \operatorname{ASExp}(\mathscr{Y})$, but equality need not hold in general if the map $u \mapsto\langle-, u\rangle: U \mapsto \operatorname{Hom}_{\mathbb{R}}(V, \mathbb{R})$ is not surjective (e.g. $\langle-,-\rangle$ is zero) or if $\mathscr{Z} \subsetneq \mathscr{Y}^{*}$ (e.g. $\left.\mathscr{Z}=\{0\} \neq \mathscr{Y}^{*}\right)$.

Special properties of the absolute notions are as follows (cf. [47, (3.6.5)] for the key fact (a) in the finite dimensional setting).

Lemma. (a) If $\operatorname{ri}(\mathscr{Y}) \neq \emptyset$, then for any $y \in \mathscr{Y} \cap \operatorname{rb}(\mathscr{Y})$, there is a proper absolutely exposed face $\mathscr{C} \subsetneq \mathscr{Y}$ of $\mathscr{Y}$ with $\mathscr{Y} y \subseteq \mathscr{C}$.

(b) If $\operatorname{ri}(\mathscr{Y}) \neq \emptyset$, then $\mathscr{Y} \cap \operatorname{rb}(\mathscr{Y})$ is the union of the proper, absolutely exposed faces of $\mathscr{Y}$.

(c) If $\operatorname{dim}(V)$ is finite and $\mathscr{C}$ is a non-empty proper face of $\mathscr{Y}$, there is a sequence $\mathscr{C}=\mathscr{C}_{0}, \ldots, \mathscr{C}_{n}=\mathscr{Y}$ of faces of $\mathscr{Y}$ such that for $i=1, \ldots, n$, $\mathscr{C}_{i-1}$ is a proper, absolutely exposed face of $\mathscr{C}_{i}$.

Proof. The proof of (a) easily reduces to the case in which aff( $\mathscr{Y})=V$. Then $\mathscr{Y}$ has an algebraically interior point $u$. By (A.3.4 and 1, Corollary 1.7], there is an affine hyperplane which separates $\operatorname{ri}\left(\mathscr{Y}_{u}\right)$ and $\operatorname{ri}\left(\mathscr{Y}_{y}\right)$ i.e some non-zero $f \in$ $\operatorname{Hom}_{\mathbb{R}}(V, \mathbb{R})$ and $a \in \mathbb{R}$ such that $f\left(\operatorname{ri}\left(\mathscr{Y}_{y}\right)\right) \subseteq \mathbb{R}_{\leq a}$ and $f\left(\operatorname{ri}\left(\mathscr{Y}_{u}\right)\right) \subseteq \mathbb{R}_{\geq a}$. Since $\operatorname{ri}\left(\mathscr{Y}_{u}\right)$ is algebraically open, we have $f\left(\operatorname{ri}\left(\mathscr{Y}_{u}\right)\right) \subseteq \mathbb{R}_{>a}$ Also, $f\left(\operatorname{cl}\left(\mathscr{Y}_{Y}\right)\right) \subseteq \mathbb{R}_{\leq a}$ and $f\left(\operatorname{cl}\left(\mathscr{Y}_{u}\right)\right) \subseteq \mathbb{R}_{\geq a}$ since $f$ is continuous. Since $\left.\left.0 \in \operatorname{cl}\left(\mathscr{Y}_{y}\right)\right) \subseteq \operatorname{cl}\left(\mathscr{Y}_{u}\right)\right)$, this gives $a=0, f\left(\mathscr{Y}_{y}\right)=\{0\}$ and $\{0\} \neq f\left(\mathscr{Y}_{u}\right) \subseteq \mathbb{R}_{\geq 0}$. Hence $\mathscr{C}:=\mathscr{Y} \cap \operatorname{ker} f$ is as required for (a). Part (b) follows from (a) and A.3.7). Part (c) follows from (a) using A.3.10 A.3.12.

A.8. Suppose henceforward in this section that $U, V$ are finite dimensional and that $\langle-,-\rangle$ is non-singular. The flc and weak topologies on $V, U$ are therefore their standard topologies as finite dimensional real vector spaces.

Consider a semidual pair $P=(\mathscr{Y}, \mathscr{Z})$ of pointed cones with respect to $\langle-,-\rangle$. Then $\operatorname{Ext}_{\neq \emptyset}(\mathscr{Y}) \supseteq \operatorname{ASExp}(\mathscr{Y}) \supseteq \operatorname{SExp}_{P}(\mathscr{Y})$. Here, $\operatorname{ASExp}(\mathscr{Y})=\operatorname{AExp}(\mathscr{Y})$ and $\operatorname{SExp}_{P}(\mathscr{Y})=\operatorname{Exp}_{P}(\mathscr{Y})$ by $(\bar{A} \cdot 6.2)$. If $\mathscr{Z}=\mathscr{Y}^{*}$, then (using the the natural isomorphism $U \rightarrow \operatorname{Hom}_{\mathbb{R}}(V, \mathbb{R})$ given by $u \mapsto\langle-, u\rangle$ to identify $U$ with the dual space of $V)$, one sees that $\operatorname{ASExp}(\mathscr{Y})=\operatorname{SExp}_{P}(\mathscr{Y})$, but trivial examples show one may have $\operatorname{ASExp}(\mathscr{Y}) \neq \operatorname{SExp}_{P}(\mathscr{Y})$ if $\mathscr{Z} \subsetneq \mathscr{Y}^{*}$.

The following example, which arises by homogenizing a standard example (see [51, Fig 2.11]) of a convex set with an extreme point which is not exposed, shows that even if $\mathscr{Z}=\mathscr{Y}^{*}$ and $\mathscr{Y}=\mathscr{Z}^{*}$, one may have $\operatorname{Ext}_{\neq \emptyset}(\mathscr{Y}) \neq \operatorname{ASExp}(\mathscr{Y})$.

Example. Let $U=V=\mathbb{R}^{3}$ with $\langle-,-\rangle$ given by the standard (dot) inner product. Let $Y:=\left\{(x, y, 1) \in \mathbb{R}^{3} \mid-1 \leq y \leq 1,-1-\sqrt{1-y^{2}} \leq x \leq 1+\sqrt{1-y^{2}}\right\}, \mathscr{Y}:=$ $\mathbb{R}_{\geq 0} Y, Z:=\left\{(x, y, 1) \in \mathbb{R}^{3} \mid-\left(1-y^{2}\right) \leq 2 x \leq 1-y^{2}\right\}$ and $\mathscr{Z}:=\mathbb{R}_{\geq 0} Z$. Then one can check that $(\mathscr{Y}, \mathscr{Z})$ is a semidual pair of stable salient cones (so $\mathscr{Y}=\mathscr{Z}^{*}$ and $\left.\mathscr{Z}=\mathscr{Y}^{*}\right)$, that $\operatorname{Ext}_{\neq \emptyset}(\mathscr{Z})=\operatorname{SExp}_{P \text { tr }}(\mathscr{Z})$ and that $\operatorname{Ext}_{\neq \emptyset}(\mathscr{Y}) \neq \operatorname{SExp}_{P}(\mathscr{Y})$. 
A.9. Condition (ii) in the following definition isolates a rather subtle special property of a semidual pair of cones which plays an important role in Section 11.

Definition. A semidual pair $P=(\mathscr{Y}, \mathscr{Z})$ of pointed cones (with respect to $\langle-,-\rangle$ ) is a dual pair of cones if the following conditions hold:

(i) $\mathscr{Y}^{*}=\mathscr{Z}^{* *}$ (equivalently, $\mathscr{Z}^{*}=\mathscr{Y}^{* *}$ ).

(ii) $\operatorname{Ext}_{\neq \emptyset}(\mathscr{Y})=\operatorname{SExp}_{P}(\mathscr{Y})$ and $\operatorname{Ext}_{\neq \emptyset}(\mathscr{Z})=\operatorname{SExp}_{P}$ tr $(\mathscr{Z})$.

The discussion in A.8 shows that if $P$ is a dual pair, then $\operatorname{Ext}_{\neq \emptyset}(\mathscr{Y})=\operatorname{ASExp}(\mathscr{Y})$ and $\operatorname{Ext}_{\neq \emptyset}(\mathscr{Z})=\overline{\operatorname{ASExp}}(\mathscr{Z})$. Example A.8 shows that even a semidual pair $(\mathscr{Y}, \mathscr{Z})$ of stable (i.e closed) cones need not be a dual pair in the above sense. In our principal applications, $\mathscr{Y}$ and $\mathscr{Z}$ are not necessarily closed.

A.10. A cone $\mathscr{Y}$ in $V$ is said to be polyhedral if $\mathscr{Y}=\mathbb{R}_{\geq 0} \Gamma$ for some finite subset $\Gamma \subseteq V$. Standard properties of polyhedral cones show they provide examples of dual pairs of stable cones.

Lemma. Let $\mathscr{Y}$ be a polyhedral cone in $V$. Then

(a) $\mathscr{Z}:=\mathscr{Y}^{*}$ is a polyhedral cone with $\mathscr{Z}^{*}=\mathscr{Y}$.

(b) $\mathscr{Y}$ and $\mathscr{Z}$ are closed and have finitely many faces.

(c) Every face of $\mathscr{Y}$ or $\mathscr{Z}$ is a polyhedral cone.

(d) Every face of $\mathscr{Y}$ is an exposed face and every face of a face of $\mathscr{Y}$ is a face of $\mathscr{Y}$. Similarly for $\mathscr{Z}$.

(e) If $\mathscr{Y}, \mathscr{Z}$ are pointed, then $(\mathscr{Y}, \mathscr{Z})$ is a dual pair of stable cones.

Proof. Parts (a)-(d) are standard, and they imply (e) by the definition of a dual pair of cones.

A.11. Part (a) of the Lemma below recalls a standard correspondence between compact convex sets and closed salient cones, while part (b) states analogues for cones of Minkowski's and Straszewicz' Theorems on compact convex sets.

Lemma. Let $\mathscr{Y}$ be a non-empty cone in $V$.

(a) The following conditions are equivalent:

(i) $\mathscr{Y}$ is closed and salient.

(ii) $\mathscr{Y}$ is closed and $\mathscr{Y}^{*}$ is generating..

(iii) $\mathscr{Y}$ is closed and $\mathscr{Y}^{*}$ has an interior point in $U$.

(iv) $\mathscr{Y}=\mathscr{Z}^{*}$ for some $\mathscr{Z} \subseteq U$ which has an interior point in $U$.

(v) There is an affine subset $H$ of $V$ with $0 \notin H$ such that $H \cap \mathscr{Y}$ is a compact (necessarily convex) base of $\mathscr{Y}$.

(vi) There exists some compact convex base $B$ of $\mathscr{Y}$.

(b) Let $\mathscr{Y}$ be a closed salient cone in $V$. Then $\mathscr{Y}=\mathbb{R}_{>0} \Gamma=\operatorname{cl}\left(\mathbb{R}_{>0} \Gamma^{\prime}\right)$ where $\Gamma$ (resp., $\Gamma^{\prime}$ ) is the union of the extreme (resp., exposed) rays of $\mathscr{Y}$. Further, $\Gamma$ is the minimum (under inclusion) union of rays of $\mathscr{Y}$ with $\mathscr{Y}=\mathbb{R}_{\geq 0} \Gamma$.

Remarks. If $V \neq 0$ and $\mathscr{Z}$ is as in (a)(iii), then (iv) is satisfied by taking $H$ to be the affine hyperplane $H=\{v \in V \mid\langle v, u\rangle=1\}$, where $u$ is any interior point of $\mathscr{Z}$. In that case, the sets $U_{\epsilon}:=\{v \in \mathscr{Y} \mid\langle v, u\rangle<\epsilon\}$ form a basis of neighborhoods of 0 in $\mathscr{Y}$, with compact closures $\overline{U_{\epsilon}}=\{v \in \mathscr{Y} \mid\langle v, u\rangle \leq \epsilon\}$.

Proof. For (a), [23, Exercise 2.13], [1, 8.6], and [5, Ch II, §7, no. 3, esp. Exemples 1]. For (b), take a base $B=H \cap \mathscr{Y}$ as in (a)(v). Then the extreme rays of $\mathscr{Y}$ are the rays spanned by the extreme points of $B$ (see [1, 8.4]), and the assertions 
involving them follows from Minkowski's theorem (i.e. the sharp form of the finite dimensional Krein-Milman theorem; see [1, Thorem (3.3)]). The other part of (b) follows similarly from Straszewicz' Theorem (see [51, Theorem 2.6.21] and [23, Exercise 3.14]).

A.12. Let $\mathscr{Y}$ be a closed salient cone in $V$ and let $B$ be a compact convex base of $\mathscr{Y}$. The map $\mathscr{U} \rightarrow K:=\mathscr{U} \cap B$ induces a bijection between the subsets of $\mathscr{Y}$ which are pointed, possibly non-convex cones, and the subsets $K$ of $B$. The inverse bijection is given by $K \mapsto \mathscr{U}=\mathbb{R}_{\geq 0} K$. Useful properties of this correspondence are listed below.

Lemma. Let $\mathscr{U} \subseteq \mathscr{Y}$ be a pointed, possibly non-convex cone, and set $K:=\mathscr{U} \cap B$.

(a) $\operatorname{cl}(\mathscr{U})$ is a pointed, possibly non-convex cone satisfying $\operatorname{cl}(\mathscr{U}) \cap B=\operatorname{cl}(K)$.

(b) $\operatorname{conv}(\mathscr{U})=\mathbb{R}_{>0} \mathscr{U}$ is a pointed cone satisfying $\mathbb{R}_{\geq 0} \mathscr{U} \cap B=\operatorname{conv}(K)$.

(c) $K^{\prime}:=\operatorname{cl}(\operatorname{conv}(K))=\operatorname{conv}(\operatorname{cl}(K))$.

(d) $\mathscr{U}^{\prime}:=\operatorname{cl}\left(\mathbb{R}_{\geq 0} \mathscr{U}\right)=\mathbb{R}_{\geq 0} \operatorname{cl}(\mathscr{U})$.

(e) $\mathscr{U}^{\prime} \cap K=\bar{K}^{\prime}$.

Proof. The straightforward proofs of (a)-(b) are omitted. Compactness of $\mathrm{cl}(K) \subseteq$ $B$ implies, by a standard consequence ([1, Ch I, Corollary (2.4)]) of Carathéodory's theorem, that $\operatorname{conv}(\operatorname{cl}(K))$ is compact. The second equality in (c) therefore holds since both its sides are the inclusion-smallest closed convex set containing $K$. By (a)-(b), $\operatorname{cl}\left(\mathbb{R}_{\geq 0} \mathscr{U}\right)$ and $\mathbb{R}_{\geq 0} \operatorname{cl}(\mathscr{U})$ are pointed cones in $\mathscr{Y}$. By (a)-(c), these two cones have the same intersection $K^{\prime}$ with $K$. The rest of the lemma follows.

\section{REFERENCES}

[1] Alexander Barvinok. A course in convexity, volume 54 of Graduate Studies in Mathematics. American Mathematical Society, Providence, RI, 2002.

[2] Anders Björner and Francesco Brenti. Combinatorics of Coxeter groups, volume 231 of Graduate Texts in Mathematics. Springer, New York, 2005.

[3] Cédric Bonnafé and Matthew J. Dyer. Semidirect product decomposition of Coxeter groups. Comm. Algebra, 38(4):1549-1574, 2010.

[4] N. Bourbaki. Éléments de mathématique. Fasc. XXXIV. Groupes et algèbres de Lie. Chapitre IV: Groupes de Coxeter et systèmes de Tits. Chapitre V: Groupes engendrés par des réflexions. Chapitre VI: systèmes de racines. Actualités Scientifiques et Industrielles, No. 1337. Hermann, Paris, 1968.

[5] Nicolas Bourbaki. Espaces vectoriels topologiques. Chapitres 1 à 5. Masson, Paris, new edition, 1981. Éléments de mathématique. [Elements of mathematics].

[6] Brigitte Brink and Robert B. Howlett. A finiteness property and an automatic structure for Coxeter groups. Math. Ann., 296(1):179-190, 1993.

[7] Brigitte Brink and Robert B. Howlett. Normalizers of parabolic subgroups in Coxeter groups. Invent. Math., 136(2):323-351, 1999.

[8] Arne Brøndsted. An introduction to convex polytopes, volume 90 of Graduate Texts in Mathematics. Springer-Verlag, New York, 1983.

[9] Roger W. Carter. Finite groups of Lie type. Wiley Classics Library. John Wiley \& Sons Ltd., Chichester, 1993.

[10] B. A. Davey and H. A. Priestley. Introduction to lattices and order. Cambridge University Press, New York, second edition, 2002.

[11] Pierre de la Harpe. Groupes de Coxeter infinis non affines. Exposition. Math., 5(1):91-96, 1987.

[12] Vinay V. Deodhar. On the root system of a Coxeter group. Comm. Algebra, 10(6):611-630, 1982.

[13] M. J. Dyer. Hecke Algebras and Reflections in Coxeter Groups. PhD thesis, Univ. of Sydney, 1987. 
[14] M. J. Dyer. Quotients of twisted Bruhat orders. J. Algebra, 163(3):861-879, 1994.

[15] M. J. Dyer. Rank two detection of singularities of Schubert varieties. Unpublished manuscript, http://www.nd.edu/ dyer/papers/index.html, 2001.

[16] M. J. Dyer. On rigidity of abstract root systems of Coxeter groups. arXiv:1011.2270 [math.GR], 2010.

[17] Matthew Dyer. Reflection subgroups of Coxeter systems. J. Algebra, 135(1):57-73, 1990.

[18] Matthew Dyer. Reflection subgroups of Coxeter systems. J. Algebra, 135(1):57-73, 1990.

[19] Matthew Dyer. On the "Bruhat graph" of a Coxeter system. Compositio Math., 78(2):185-191, 1991.

[20] Matthew Dyer. On parabolic closures in Coxeter groups. J. Group Theory, 13(3):441-446, 2010.

[21] Matthew Dyer, Christophe Hohlweg, and Vivien Ripoll. Asymptotical behaviour of roots of infinite Coxeter groups ii. In preparation, 2012.

[22] Tom Edgar. Dominance and regularity in Coxeter groups. ProQuest LLC, Ann Arbor, MI, 2009. Thesis (Ph.D.)-University of Notre Dame.

[23] Monique Florenzano and Cuong Le Van. Finite dimensional convexity and optimization, volume 13 of Studies in Economic Theory. Springer-Verlag, Berlin, 2001. In cooperation with Pascal Gourdel.

[24] Xiang Fu. The dominance hierarchy in root systems of Coxeter groups. arXiv:1108.2940 [math.RT], 2010.

[25] Xiang Fu. Coxeter groups, imaginary cones and dominance. arXiv:1108.5232 [math.RT], 2011.

[26] Xiang Fu. Non-orthogonal geometric realizations of Coxeter groups. arXiv:1112.3429 [math.RT], Preprint, 2011.

[27] Helge Glöckner. Positive definite functions on infinite-dimensional convex cones. Mem. Amer. Math. Soc., 166(789):xiv+128, 2003.

[28] J.-Y. Hée. Le cône imaginaire d'une base de racines sur $\mathbb{R}$. Unpublished manuscript.

[29] J.-Y. Hée. Sur la torsion de Steinberg-Ree des groupes de Chevalley et des groupes de KacMoody. PhD thesis, Université de Paris-Sud, Orsay, 1993.

[30] Christophe Hohlweg, Jean-Phillipe Labbé, and Vivien Ripoll. Asymptotical behaviour of roots of infinite Coxeter groups I. arXiv:1112.5415v2 [math.GR], 2012.

[31] R. B. Howlett, P. J. Rowley, and D. E. Taylor. On outer automorphism groups of Coxeter groups. Manuscripta Math., 93(4):499-513, 1997.

[32] James E. Humphreys. Reflection groups and Coxeter groups, volume 29 of Cambridge Studies in Advanced Mathematics. Cambridge University Press, Cambridge, 1990.

[33] Victor G. Kac. Infinite-dimensional Lie algebras. Cambridge University Press, Cambridge, 1990.

[34] Daan Krammer. The conjugacy problem for Coxeter groups. Groups Geom. Dyn., 3(1):71$171,2009$.

[35] Eduard Looijenga. Invariant theory for generalized root systems. Invent. Math., 61(1):1-32, 1980.

[36] G. A. Margulis and È. B. Vinberg. Some linear groups virtually having a free quotient. $J$. Lie Theory, 10(1):171-180, 2000.

[37] George Maxwell. The normal subgroups of finite and affine Coxeter groups. Proc. London Math. Soc. (3), 76(2):359-382, 1998.

[38] C. Mokler. Die Monoidvervollständigung einer Kac-Moody-Gruppe, dissertation. PhD thesis, Hamburg, 1996.

[39] C. Mokler. An algebraic geometric model of an action of the face monoid associated to a Kac-Moody group on its building. arXiv:0906.5059 [math.RT], 2009.

[40] Claus Mokler. An analogue of a reductive algebraic monoid whose unit group is a Kac-Moody group. Mem. Amer. Math. Soc., 174(823):vi+90, 2005.

[41] Claus Mokler. Actions of the face monoid associated to a Kac-Moody group on its building. J. Algebra, 321(9):2384-2421, 2009.

[42] Koji Nuida. Parabolic subgroups in Coxeter groups of arbitrary ranks. arXiv:1106.4709 [math.GR], 2011.

[43] Werner C. Rheinboldt and James S. Vandergraft. A simple approach to the Perron-Frobenius theory for positive operators on general partially-ordered finite-dimensional linear spaces. Math. Comp., 27:139-145, 1973. 
[44] R. Tyrrell Rockafellar. Convex analysis. Princeton Landmarks in Mathematics. Princeton University Press, Princeton, NJ, 1997. Reprint of the 1970 original, Princeton Paperbacks.

[45] P. Slodowy. SingularitätenKac-Moody-Liealgberen und assoziierte Gruppen, Habilitationsschrift. PhD thesis, Bonn, 1984.

[46] Peter Slodowy. An adjoint quotient for certain groups attached to Kac-Moody algebras. In Infinite-dimensional groups with applications (Berkeley, Calif., 1984), volume 4 of Math. Sci. Res. Inst. Publ., pages 307-333. Springer, New York, 1985.

[47] Josef Stoer and Christoph Witzgall. Convexity and optimization in finite dimensions. I. Die Grundlehren der mathematischen Wissenschaften, Band 163. Springer-Verlag, New York, 1970.

[48] James S. Vandergraft. Spectral properties of matrices which have invariant cones. SIAM J. Appl. Math., 16:1208-1222, 1968.

[49] È. B. Vinberg. Discrete linear groups that are generated by reflections. Izv. Akad. Nauk SSSR Ser. Mat., 35:1072-1112, 1971.

[50] Mark E. Watkins. Infinite paths that contain only shortest paths. J. Combin. Theory Ser. B, 41(3):341-355, 1986.

[51] Roger Webster. Convexity. Oxford Science Publications. The Clarendon Press Oxford University Press, New York, 1994.

[52] Nanhua Xi. Lusztig's A-function for Coxeter groups with complete graphs. Bull. Inst. Math. Acad. Sin. (N.S.), 7(1):71-90, 2012.

Department of Mathematics, 255 Hurley Building, University of Notre Dame, Notre DAme, Indiana, 46556-4618, U.S.A.

E-mail address: dyer.1@nd.edu 